University of Rhode Island

DigitalCommons@URI

Open Access Dissertations

1987

\title{
Optimization of Solid/Liquid Separation Processes Using Particle Size Distribution Analysis
}

Abdulrhaman Ibrahim Alabdulaly

University of Rhode Island

Follow this and additional works at: https://digitalcommons.uri.edu/oa_diss

\section{Recommended Citation}

Alabdulaly, Abdulrhaman Ibrahim, "Optimization of Solid/Liquid Separation Processes Using Particle Size Distribution Analysis" (1987). Open Access Dissertations. Paper 549.

https://digitalcommons.uri.edu/oa_diss/549

This Dissertation is brought to you for free and open access by DigitalCommons@URI. It has been accepted for inclusion in Open Access Dissertations by an authorized administrator of DigitalCommons@URI. For more information, please contact digitalcommons@etal.uri.edu. 
OPTIMIZATION OF SOLID/LIQUID SEPARATION PROCESSES

USING PARTICLE SIZE DISTRIBUTION ANALYSIS

BY

ABDULRABRAN IBRAHIM ALABDULALY

A DISSERTATION SUBMITTED IN PARTIAL FULFILLMENT OF THE

REQUIREHENTS FOR THE DEGREE OF

DOCTOR OF PHILOSOPHY

IN

CIVIL AND ENVIRONMENTAL ENGINEERING

UNIVERSITY OF RHODE ISLAND

1987 


\section{DOCTOR OF PHILOSOPHY DISSERTATION}

OF

\section{ABDULRAHMAN IBRAHIM ALABDULALY}

Approved:

Dissertation Committee

Major Professor

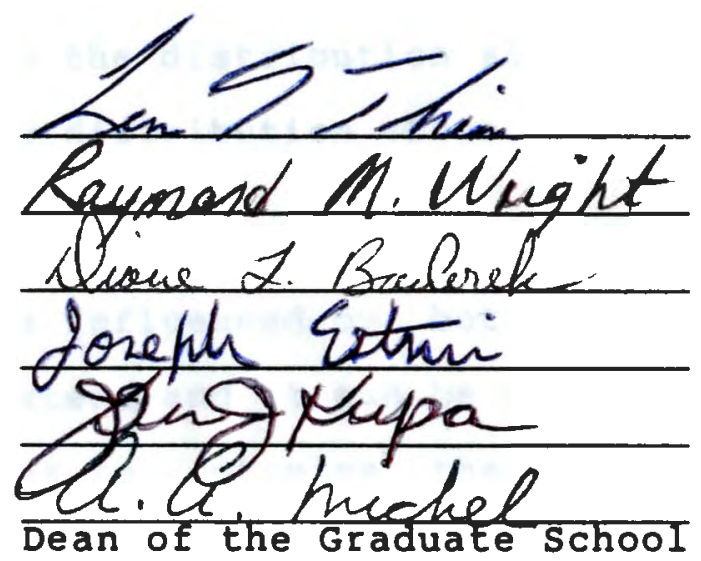

UNIVERSITY OF RHODE ISLAND

1987 


\section{ABSTRACT}

A study was conducted to investigate the influence of the coagulant dosage, suspension $\mathrm{pH}$, mixing intensity and time on the suspended solids particle size distribution during coagulation, flocculation and filtration of dilute suspensions. The coagulation/flocculation investigation consisted of laboratory experiments utilizing the jar test apparatu's as well as a pilot plant mixing tank. Filtration experiments were conducted using $3-s i x$ inch inside diameter glass columns with different media sizes. The data generated were used to compute the distribution slope of the particles in each sample. The distribution slope was used as the pretreatment performance indicator.

The distribution slope is influenced by both chemical and physical treatment parameters and it can be used in the treatment of dilute suspensions to optimize the coagulant dosage, mixing intensity, and mixing time.

Both the total number of particles and the distribution slope of flocculated suspensions were found to follow a second order polynomial equation due to floc breakage after the optimum mixing time. 
The coagulant dosage was found to affect both the head loss development and the effluent quality of granular media filters. The mixing intensity and time were found to influence mostly the head loss development and not the effluent quality. The effluent quality improved with the increase in the coagulant dosage and mixing time and deteriorated with the increase in the mixing intensity.

The filter's effluent particle size distribution was influenced by both the influent particle size distribution and media size. Fine media was sensitive to the influent changes whereas coarse media showed much less sensitivity to changes in the influent characteristics. 


\section{ACRNOWLEDGMENTS}

My sincere appreciation and thanks go to my advisor Dr. Leon T. Thiem for the direction and supervision that he has given me all along. I would also like to thank Dr. Raymond M. Wright for his valuable comments, suggestions and the time he spent reviewing the manuscript. special thanks to Dr. Diane L. Badorek and Dr. Joseph Estrin for their review and comments.

I would also like to thank Milton $T$. Huston and George w. Huston for their help and patience in the construction of the pilot plant. Gratitude is extended to graduate students and friends Talal A. Radain and Blas J. Gomez for their assistance in the writing of the computer programs, and to George Chiu for his excellent work on the graphics.

Finally, I thank my family, especially my father Ibrahim and my wife Huda for their support and encouragement. 


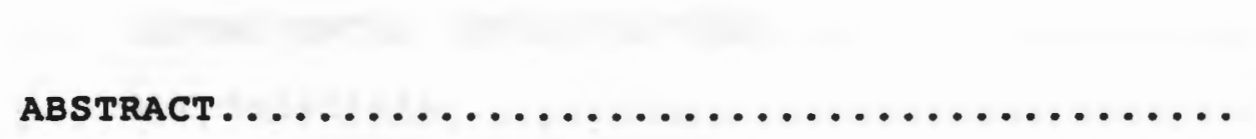

Page

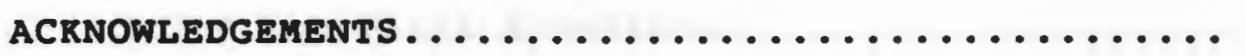

TABLE OF CONTENTS.

$1 v$

LIST OF FIGURES.

$\mathbf{v}$

LIST OF TABLES.

$v i 1$

IIST OF SYMBOLS

$x i v$

LIST OF SYMBOLS

$x \vee i$

1. INTRODUCTION .................................. 1

1.1 statement of the Problem................. 6

1.2 objectives of the study................. 11

2. LITERATURE REVIEW............................. 12

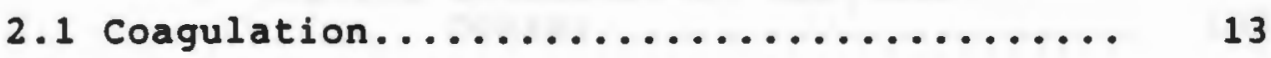

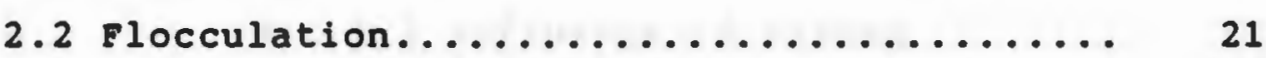

2.3 Optimization of Coagulation/Flocculation

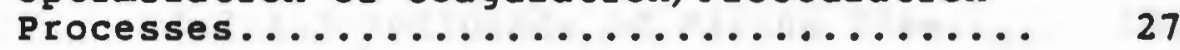

2. 4 Granular-Media Filtration.............. 31

2.4.1 Process Mechanisms................ 32

2.4.2 Variables Affecting Direct

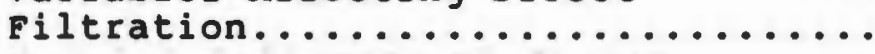

39

2.5 Particle size and Distribution...........

2.6 Influence of the Influent Particle size

Distribution on the Performance of

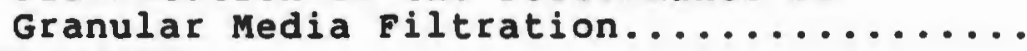

2.6.1 Influence of Coagulant Dosage...... 53

2.6.2 Influence of Mixing Intensity...... 56

2.6 .3 Influence of Mixing Time......... 57 
3. EXPERIMENTAL INVESTIGATION................. 61

3.1 Materials........................ 61

3.2 Pilot-Plant Apparatus................ 65

3.3 Experimental Procedure............... 79

4. RESULTS AND DISCUSSION................... 102

4.1 Pretreatment....................... 102

4.1 .1 Jar Test Results............... 102

4.1.2 Pilot-Plant Mixing Tank Results..... 141

4.2 Pilot Plant Filtration................. 154

4.2.1 Influence of Influent Particle size Distribution on Effluent Quality....

154

4.2.1.1 Influence of Coagulant Dosage................... 155

4.2.1.2 Influence of Mixing Intensity............... 174

4.2.1.3 Influence of Mixing Time... 186

4.2.2 Influence of Influent Particle Size Distribution on Head Loss......... 199

4.2.2.1 Influence of Coagulant Dosage................. 199

4.2.2.2 Influence of Mixing Intensity.............. 208

4.2.2.3 Influence of Mixing Time.... 213

5. ConCLUSIONS....................... 219

6. RECOMMENDATIONS...................... 221

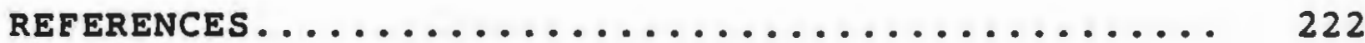
APPENDIX A:

List of the Computer Program.......... 
APPENDIX B :

$$
\begin{aligned}
& \text { Pretreatment Data } \\
& \text { - Jar Test........................... } 236 \\
& \text { - Pilot-Plant Mixing Tank...... } 248
\end{aligned}
$$

APPENDIX C :

$$
\begin{aligned}
& \text { Pilot Plant Data } \\
& \text { - Data for Filter Number 1......... } 253 \\
& \text { - Data for Filter Number 2........ } 261 \\
& \text { - Data for Filter Number 3......... } 269
\end{aligned}
$$

AP PENDIX D :

Head Loss Graphs.................. 277 APPENDIX E :

Tables of the Head Loss Rates........ 287 


\section{LIST OF FIGURES}

Page

Figure 2.1 schematic representation of the coagulation of colloidal suspensions by $\mathrm{Al}$ (III) or $\mathrm{Fe}$ (III) salts......... 18

Figure 2.2 Design and operation diagram for alum

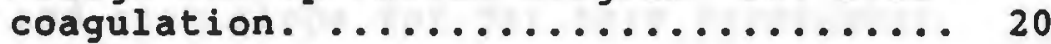

Figure 3.1 Comparison of the particle size distribution of the scituate Reservoir water with MIN-U-GEL 200 at two different mass concentrations. ....... 66

Figure 3.2 particle size distribution of MIN-U-GEL 200, MIN-U-SIL 30 and scituate Reservoir water.......... 67

Figure 3.3 schematic diagram of the pilot plant setup. ....................... 68

Figure 3.4 Detailed schematic diagram of the pilot plant pretreatment setup. ...... 70

Figure 3.5 Photograph of the pilot plant pretreatment setup............... 71

Figure 3.6 Detailed schematic of a typical pilot plant filtration column........... 74

Figure 3.7 Photograph of the pilot plant sand filtration setup. ................. 75

Figure 3.8 Flow chart of the jar test experiments 80

Figure 3.9 Total number of particles/ml with different dilution ratios of MIN-U-GEL $200 . \ldots \ldots \ldots \ldots \ldots . \ldots . \ldots . \ldots$

Figure 3.10 velocity gradient versus rpm for the pilot plant mixing tank with two different blade sizes.............. 91

Figure 3.11 Flow chart of the sand filtration runs. 93

Figure 3.12 velocity gradient versus rpm for the

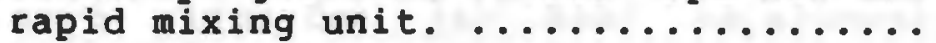


Figure 4.1 Remaining total number of particles/ml versus mixing time for jar test experiment number $1 . \ldots \ldots \ldots \ldots \ldots$

Figure 4.2 Distribution slope versus mixing time for jar test experiment number 1 ..... 106

Figure 4.3 Remaining total number of particles/ml and distribution slope versus coagulant dose at both 30 minutes mixing time and zero slope for jar test experiment number $1 . \ldots \ldots \ldots \ldots \ldots \ldots . \ldots \ldots 10 . \ldots \ldots$

Figure 4.4 Remaining total number of particles/ml versus mixing time for jar test

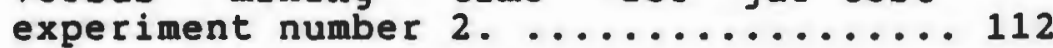

Figure 4.5 Distribution slope versus mixing time for jar test experiment number 2..... 113

Figure 4.6 Remaining total number of particles/ml and distribution slope versus coagulant dose at both 30 minutes mixing time and zero slope for jar test experiment number $2 . \ldots \ldots \ldots \ldots \ldots \ldots \ldots \ldots . \ldots \ldots 115$

Figure 4.7 Remaining total number of particles/ml versus mixing time for jar test experiment number $3 . \ldots \ldots \ldots \ldots \ldots \ldots$

Figure 4.8 Distribution slope versus mixing time for jar test experiment number 3..... 119

Figure 4.9 Remaining total number of particles/ml and distribution slope versus coagulant dose at both 30 minutes mixing time and zero slope for jar test experiment number $3 . \ldots \ldots \ldots \ldots \ldots \ldots \ldots \ldots . . . \ldots$

Figure 4.10 Remaining total number of particles/ml versus mixing time for jar test experiment number 4............... 124

Figure 4.11 Distribution slope versus mixing time for jar test experiment number 4 ..... 125

Figure 4.12 Remaining total number of particles/ml and distribution slope versus solution $\mathrm{pH}$ at both 30 minutes mixing time and zero slope for jar test experiment number $4 . \ldots \ldots \ldots \ldots \ldots \ldots . \ldots \ldots 128$ 
Figure 4.13 Remaining total number of particles/ml versus mixing time for jar test experiment number 5............... 130

Figure 4.14 Distribution slope versus mixing time for jar test experiment number 5..... 131

Figure 4.15 Remaining total number of particles/ml and distribution slope versus coagulant dose at both 30 minutes mixing time and zero slope for jar test experiment

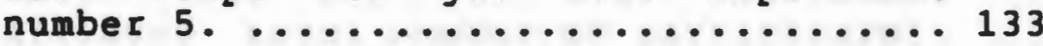

Figure 4.16 Remaining total number of particles/ml versus mixing time for jar test experiment number $6 . \ldots \ldots \ldots \ldots \ldots \ldots 136$

Figurè 4.17 Distribution slope versus mixing time for jar test experiment number $6 . \ldots 137$

Figure 4.18 Remaining total number of particles/ml and distribution slope versus coagulant dose at both 30 minutes mixing time and zero slope for jar test experiment number $6 . \ldots \ldots \ldots \ldots \ldots \ldots \ldots \ldots \ldots . . \ldots \ldots$

Figure 4.19 Remaining total number of particles/ml versus mixing time for four mixing intensities for the pilot plant mixing tank.

Figure 4.20 Distribution slope versus mixing time for four mixing intensities for the pilot plant mixing tank............. 143

Figure 4.21 Rate of flocculation versus mixing intensity based on the remaining total number of particles and the distribution slope for the pilot plant mixing tank. ................... 144

Figure 4.22 Optimum mixing time versus mixing intensity for the pilot plant mixing tank. ......................146

Figure 4.23 Remaining total number of particles/ml and distribution slope at optimum mixing time versus mixing intensity for the pilot plant mixing tank. ...... 148 
Figure 4.23 a Comparison between first and second order polynomial equations for the pilot plant mixing tank using the remaining total number of particles

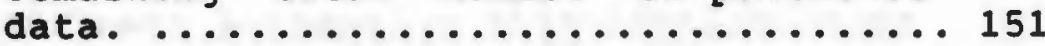

Figure $4.23 b$ Comparison between first and second order polynomial equations for the pilot plant mixing tank using the distribution slope data. ............

Figure 4.24 Effluent total number of particles/ml versus run time for fine sand with various alum dosages...............156

Figure 4.25 Effluent total number of particles/ml versus run time for medium sand with various alum dosages.............. 161

Figure 4.26 Effluent total number of particles/ml versus run time for coarse sand with various alum dosages. ............ 165

Figure 4.27 Influent suspension distribution slope versus coagulant dose for the pilot plant experiments. ................ 168

Figure 4.28 Effluent suspension distribution slope versus run time for fine sand with various alum dosages............. 169

Figure 4.29 Effluent suspension distribution slope versus run time for medium sand with various alum dosages............. 170

Figure 4.30 Effluent suspension distribution slope versus run time for coarse sand with various alum dosages............. 171

Figure 4.31 Effluent total number of particles/ml versus run time for fine sand with various mixing intensities......... 175

Figure 4.32 Effluent total number of particles/ml versus run time for medium sand with various mixing intensities......... 176

Figure 4.33 Effluent total number of particles/ml versus run time for coarse sand with various mixing intensities........ 177 
Figure 4.34 Influent suspension distribution slope versus mixing intensity for the pilot plant experiments................ 180

Figure 4.35 Effluent suspension distribution slope versus run time for fine sand with various mixing intensities......... 181

Figure 4.36 Effluent suspension distribution slope versus run time for medium sand with various mixing intensities......... 182

Figure 4.37 Effluent suspension distribution slope versus run time for coarse sand with various mixing intensities.......... 183

Figure,4.38 Effluent total number of particles/ml versus run time for fine sand with various mixing times. ........... 187

Figure 4.39 Effluent total number of particles/ml versus run time for medium sand with various mixing times.............. 189

Figure 4.40 Effluent total number of particles/ml versus run time for coarse sand with various mixing times.............. 191

Figure 4.41 Influent suspension distribution slope versus mixing time for the pilot plant experiments.

Figure 4.42 Effluent suspension distribution slope versus run time for fine sand with various mixing times. ............ 195

Figure 4.43 Effluent suspension distribution slope versus run time for medium sand with

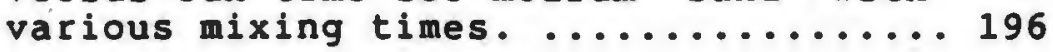

Figure 4.44 Effluent suspension distribution slope versus run time for coarse sand with various mixing times............. 198

Figure 4.45 Rate of head loss development versus alum dose for fine media at various sand depths.................... 204

Figure 4.46 Rate of head loss development versus alum dose for medium sand at various

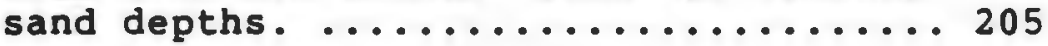


Figure 4.47 Rate of head loss development versus alum dose for coarse sand at various sand depths..................206

Figure 4.48 Rate of head loss development versus mixing intensity for fine sand at

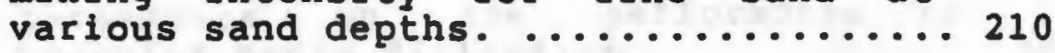

Figure 4.49 Rate of head loss development versus mixing intensity for medium sand at various sand depths. ..................211

Figure 4.50 Rate of head loss development versus mixing intensity for coarse sand at various sand depths............... 212

Figure 4.51 Rate of head loss development versus mixing time for fine sand at various

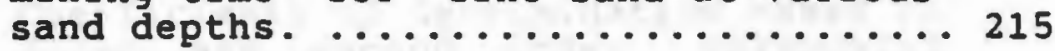

Figure 4.52 Rate of head loss development versus mixing time for medium sand at various

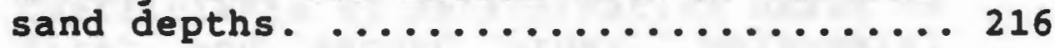

Figure 4.53 Rate of head loss development versus mixing time for coarse sand at various

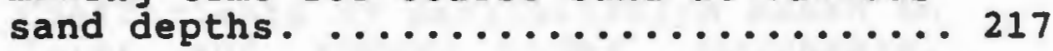




\section{LIST OF TABLES}

Page

Table 2.1 Influence of the pretreatment parameters on the performance of granular media filtration. .......... 60

Table 3.1 MIN-U-GEL 200 particle characterization 64

TABLE 3.2 Calibration of the slow mixing tank... 90

Table 3.3 University tap water particle size analysis..................... 95

Table. 3.4 Calibration of the rapid mixing unit. . 98

Table 4.1 Coefficients of determination based on first and second order polynomial functions for experiment number $1 . \ldots 110$

Table 4.2 Coefficients of determination based on first and second order polynomial functions for experiment number 2.... 110

Table 4.3 Coefficients of determination based on first and second order polynomial functions for experiment number $3 . \ldots 120$

Table 4.4 Coefficients of determination based on first and second order polynomial functions for experiment number 4.... 120

Table 4.5 Coefficients of determination based on first and second order polynomial functions for experiment number 5.... 135

Table 4.6 Coefficients of determination based on first and second order polynomial functions for experiment number 6 .... 135

Table 4.7 Coefficients of dètermination based on first and second order polynomial functions for pilot plant mixing tank.. 150

Table 4.8 Rates of effluent quality deterioration for filter number $1 . \ldots \ldots \ldots \ldots . \ldots 158$

Table 4.9 Rates of effluent quality deterioration for filter number $2 . \ldots \ldots \ldots \ldots \ldots \ldots . \ldots 163$ 
Table 4.10 Rates of effluent quality deterioration for filter number $3 . \ldots \ldots \ldots \ldots \ldots . \ldots 166$

Table 4.11 Coefficients of determination for the rates of head loss for filter number 1. 201

Table 4.12 Coefficients of determination for the rates of head loss for filter number 2. 202

Table 4.13 coefficients of determination for the rates of head loss for filter number 3. 203

Table 4.14 Coefficients of determination for the relationships between the coagulant dosage, mixing intensity, mixing time and the rate of head loss for the three 


\section{LIST OF SYMBOLS}

A - Constant related to particles concentration

$A_{p}=$ Area of the paddle blade $\left(f t^{2}\right)$

$b(i, j)=$ The collision frequency function

$\left(b_{i j}\right)_{\text {or }}=$ The collision frequency function due to orthokinetic flocculation $\left(f t^{3} s^{-1}\right)$

$\left(b_{i j}\right)_{p e}=$ The collision frequency function due to perikinetic flocculation $\left(f t^{3} s^{-1}\right)$

$\left(b_{i j}\right)_{s e}=$ The collision frequency function due to sedimentation

$C=$ Floc strength coefficient

$C_{d}=$ Drag coefficient

c = The largest particle size considered (micrometer)

$\mathrm{D}_{3}$ - Maximum stable floc diameter (micrometer)

$\mathrm{D}_{\mathrm{u}}$ = Maximum size of particle that can be formed

$d_{p}=$ Particle diameter (micrometer)

$d_{s}$ - Sand grain diameter $(\mathrm{mm})$

f = Coefficient related to the breakup mode and size regime of eddies

G - Mean velocity gradient $\left(s^{-1}\right)$

9 = Gravitational constant (ft.sec-2)

$i, j, k=$ Particular particle size

$K=$ Boltzmann's constant $\left(J^{\circ} K^{-1}\right)$

$\mathbf{k}_{1}=$ Constant

L = Particle size interval (micrometer)

$L_{8}=$ sand bed depth (ft)

$N=$ The number concentration of particles $\left(f t^{-3}\right)$ 
$N_{A}$ - Actual number of particles present in the original sample before dilution

$N_{D}$ = Number of particles present in the deionized water

$N_{p}=$ Number of particles present in the diluted sample

$n$ - speed of the shaft (rpm)

$P$ = Power input (W)

$r$ - Particle radius (micrometer)

$s$ - Surface area of the particle ( $f t^{2}$ )

$T$ - Absolute temperature $\left({ }^{\circ} \mathrm{K}\right)$

$T_{q}=$ The measured torque (lb-ft)

$t$ - Time (s)

$v$ = volume of liquid ( $\mathrm{ft}^{3}$ )

$v_{D}$ = volume of delonized water $(\mathrm{ml})$

$v_{p}=$ volume of particle (micrometer ${ }^{3}$ )

$v_{s}=$ volume of original sample (ml)

$v_{T}=$ Total volume in the sampling bottle (ml)

$v_{b}=$ Mean paddle speed (ft $s^{-1}$ )

$v_{1}$ = Mean liquid velocity (ft $s^{-1}$ )

w = Total suspension solids (grams)

$\alpha \quad=$ The collision efficiency factor

$\beta=$ The distribution slope

$\gamma_{1}=$ strength constant

$\gamma_{2}=$ Area constant for density difference

$\mu=$ Fluid absolute viscosity (lb ft $\mathrm{ft}^{-1} \mathrm{~s}^{-1}$ )

$v=$ Dynamic viscosity (1b-s ft-2)

$P_{1}=$ Density of the liquid (lb ft-3)

$\rho_{p}=$ Density of the particles (lb ft-3)

$\omega$. = Shaft rotation speed (rad s-1) 
$\eta_{D}=$ single collector efficiency due to diffusion

$\eta_{1}$ = single collector efficiency due to interception

$\eta_{s}$ = single collector efficiency due to sedimentation

$\eta_{T}$ - Total single collector efficiency 


\section{INTRODUCTION}

The purpose of treating surface waters prior to public consumption is to produce a healthful, aesthetically pleasing potable water. The major contaminants that are found in surface waters are protozoa, viruses, bacteria, asbestos, trihalomethanes (THM), precursors, algae, and particulate matter of all kind. Some contaminants are removed because of their direct effect on human health, others because of aesthetic values. Most of these contaminants are usually removed by conventional methods; chemical coagulation, sedimentation, sand filtration and disinfection. Once these contaminants are removed, then disinfection can be achieved with minimal, rather than large, doses of chlorine. Consumers can then be assured that the water is free of undesirable materials and pathogenic organisms for which direct measurement is currently impractical.

In most water treatment plants, turbidity and sometimes color are the two parameters that have been used as the performance indicators of the whole process. Turbidity is considered the most common surrogate parameter for particles, and color is the most common surrogate parameter for natural organic material. The United states Environmental Protection Agency (U.S. EPA) has established a maximum concentration limit of 1.0 nephelometric turbidity 
unit (NTU) for public water supplies. The American water Works Association's (AWWA) goal for maximum turbidity in public drinking waters is 0.10 NTU. These criteria are based principally on health as well as aesthetic considerations.

Particles present in waters have a range of impacts on water quality depending on their physical, chemical and biological properties. These particles span a great range of sizes, shapes and structures. Because of their fine size, particles exhibit a large surface area to volume ratio which can serve as a potential adsorption sink for toxic substances, such as heavy metals and chlorinated hydrocarbons. Past studies have shown that bacteria and viral agents are attached to organic and inorganic particulates causing ineffective disinfection. Therefore ingestion of particles, if contaminated, may then cause acute or chronic toxic effects. Also particulate materials responsible for turbidity have the capability of shielding viruses from the disinfection agents (Tate et al. 1978). Moreover, particulate matter is believed to serve as carrier for nutrients that can result in biological activity downstream from the treatment plant (McCoy et al. 1986).

Bitton et al. (1974a,b) incubated viruses in natural seawater and in natural seawater plus a clay suspension. The initial inoculum was $5 \times 10^{7}$ virus particles per milliliter of seawater. After 60 days of incubation, the survival of viruses was less than 10 virus particles per 
milliliter for the seawater alone, and $3 \times 10^{4}$ virus particles per milliliter for the seawater plus the clay particles. Bitton et al. concluded that the protective effect exerted by the colloids was probably a result of the adsorption of viruses onto the surfaces of these colloids. The virus may be protected either by adsorption to inorganic or organic colloids, or by inactivation of anti-viral chemicals on the colloid surface.

Most of the naturally occurring suspended solids found in water include clays, organic matter, metal oxides and minerals such as silica. Particles occurring in both natural waters and wastewaters vary in diameter from 0.005 to about 100 micrometers $\left(5 \times 10^{-9}\right.$ to $\left.10^{-4} \mathrm{~m}\right)$ (stumm 1977 . o'Connell 1978, and Dempsey et al. 1984b). Most of the bacteria found in natural waters have a size in the range of 0.3 to 10 micrometers (Beard et al. 1977). Some microorganisms such as bacteria are removed effectively by chlorination, others which have more resistance such as the spores and cysts of some species are not removed by chlorination alone but must be removed by other processes such as coagulation and filtration.

Effective removal of particles from raw water requires knowledge of the size distribution and concentration of such particles. Depending upon the solids concentrations and the average particle size, there are basically three types of treatment processes that are used in the removal of particles from surface water. These three types of 
treatment are:

(1) Contact filtration, in which destabilizing chemicals are added to the raw water and the resulting destabilized suspension is applied to the filter without further processing;

(2) direct filtration, in which the destabilized suspension is flocculated to accomplish particle growth prior to filtration; and

(3) conventional treatment, in which destabilization, flocculation, and sedimentation precede filtration.

Contact filtration is optimal at very low particle concentrations (less than $5-10 \mathrm{mg} / \mathrm{L}$ ). This is because excessively long flocculation periods are needed to produce particle aggregation in such dilute suspensions. Direct filtration is economically attractive when the mass concentration of particles in the raw water supply is less than 15 to $20 \mathrm{mg} / \mathrm{L}$. Flocculation in this process provides sufficient particle growth to reduce the head loss and breakthrough caused by small particles. Conventional treatment is usually practiced when the mass concentrations of the particles in the raw water is greater than $20 \mathrm{mg} / \mathrm{L}$. This is because particles are removed at less cost by adding settling tanks than by building larger filters to remove the high solids loading. 
Direct filtration has been used successfully in treating surface waters with turbidities ranging from 0.1 to 60 NTU. The widespread use of direct filtration stems from the cost savings that are associated with the process. The cost savings in direct filtration plants are the results of savings on building and operation expenses. The omission of large settling basins results in lower plant construction costs- and possible savings on land cost. Elimination of settling basins can yield a capital cost savings of 20 to $30 \%$ (Sweeney et al. 1974, Culp 1977, Tate et al. 1977, and Logsdon 1978). Additional savings stem from being able to effectively treat the water by utilizing a 10 to 30 \% reduction in chemical doses, having less equipment (no settling basin sludge removal equipment) to operate and maintain; and by having less sludge to treat as a result of lower chemical doses.

There are several disadvantages of direct filtration. The capital savings from elimination of settling basins can be slightly offset by a reduced length of filter runs. The average filter run in conventional treatment exceeds 50 hours whereas in direct filtration the average is less than 30 hours. Because all suspended solids are removed in the filter, practical upper limits exist on the turbidity, color, and algae that can be present in a raw water without causing very short filter runs. Wash water usage may be as high as 6 per cent of the product volume in direct filtration. In conventional treatment the value is 4 per 
cent for the treatment of the same raw water.

A major disadvantage of direct filtration is the short residence time of water in the plant. The residence time could be as short as one-half hour and the plant operator must watch for influent water quality changes that could cause effluent quality degradation. Shorter residence times also means less contact time for disinfection.

\section{1 statement of the Problem}

The removal of suspended particles from the raw water requires an optimization of the processes involved to assure a high quality potable water at the lowest cost. In direct filtration, three processes must be optimized. The chemical dose and pH are usually optimized in the coagulation process, the mixing time and intensity are optimized in the flocculation process, and the filter run length and final effluent quality are optimized in the filtration process.

Traditionally, one of the primary indicators of water treatment effectiveness has been turbidity. Reductions in turbidity or attainment of a given turbidity objective has historically been studied in pilot plants and full-scale plants. However, turbidity is an indirect measurement of particulates based on light scattering. Scattering is a function of several factors such as particle shape, size, refractive index, and number. Turbidity measurements are determined by the total light scattered by particles. This 
gives no information on the particle size and distribution present in the sample. It is possible that two different samples could have the same turbidity measurements but a different particle size distribution. There is no single relationship between turbidity and particle size distribution. Treweek et al. (1980) concluded that before flocculation, more than 908 of suspension turbidity occurs in particles smaller than 2 micrometers, whereas after flocculation, more than 808 of the residual turbidity occurs in particles greater than 2 micrometers in diameter. silverman et al. (1983) found that turbidity is closely associated with particle sizes around 2-40 micrometers.

Particle counts, in contrast to turbidity measurements, provide a direct measure of the particulate matter present in the water and its size distribution. The particle size distribution can be used to record the growth of aggregates in the flocculation step and to predict their subsequent removal by direct filtration. Particle counting is a more sensitive analytical technique than turbidity for measuring the concentration of suspended solids greater than 1 micrometer in particle diameter or for raw water with low turbidity values (less than 5 turbidity units) (Kavanaugh et al. 1980, and Monscritz et al. 1983).

According to Tate et al.(1978) and Hutchinson (1985), particle counting has the following advantages over turbidity measurements:

(1) a direct measurement of particulate material is 
provided,

(2) the sensitivity of suspended solids measurements is enhanced,

(3) there exists a potential for improved operational control, and

(4) a greater guarantee of disinfection.

The particulate size frequency distribution in natural waters has been shown to follow very closely the power-law size distribution function. The coagulation and flocculation processes cause the particle size distribution (PSD) of the raw water to change. The change is reflected in both the change in the remaining particles as well as the size of these particles. The size of the remaining particles is represented by the slope of the distribution function. With treatment, either physical, chemical or both, the slope of the distribution function decreases up to a certain value. Therefore the slope of the distribution function can be used as a performance indicator.

Compared to the total number of particles, the slope of the distribution function provides more information about the contribution of different particle sizes present in the sample to the total particle count. The size of particles in the suspension is more important in the removal processes than the total number. Therefore, instead of identifying each size and their corresponding count or using the total particle count in a certain size range, the slope of the distribution function can be used as a common 
representative.

The slope of the distribution function as a possible performance indicator has not been investigated fully in the past. The change of this slope, which occurs after the pretreatment (coagulation and flocculation) steps has not been studied in detail. Moreover, the distribution of particles in the filter effluent with different influent characteristics has not been investigated in a pilot plant scale before. The influence of the influent particle size distribution to granular media filtration on the effluent quality and head loss development has not been studied in detail.

In filtration, the last four decades have witnessed very extensive efforts to develop the basics of the theory of filtration by investigators all over the world. The investigators include Mintz and Shekhtman in Russia, the Marckrle brothers in Czechoslovakia, Lerk in Holland, Ives and his students in England, Deb and Sakthivadivel in India, and Camp, O'Melia, Cleasby, Baumann and Stumm in the United states. Most of the theories dealt primarily with the physical aspect of filtration by trying to predict the changes in head loss and concentration of suspended matter throughout the course of a filter run. The theorists have disagreed among themselves to the extent that the use of the models was only applicable to the investigator's own experimental conditions. 
Previous direct filtration research has usually been limited to studying the effect of only one or two operating parameters. The reasons for such limitations are time and cost. Pilot scale studies can be labor-intensive and time-consuming and can involve extensive and costly equipment. Instead, bench-scale studies have been used extensively in the coagulation, flocculation and filtration processes. The results obtained are limited in application but they can be useful in the elimination of relatively ineffective variables for subsequent pilot-plant testings.

The use of particle size analysis has been limited due to the cost associated with analytical equipment and the time required for data analysis. Moreover the use of particle size distribution analysis in water treatment plants has not received great attention due to the lack of information about its importance. Despite these advantages, the use of on-line particle counting in treatment plants has been shown to be cost effective. Hutchinson (1985) reported as much as $30 \%$ reduction in the chemical costs was achieved when particle counting was incorporated in the 390 million gallon per day direct filtration plant supplying the las Vegas area. Because particle counters have the capability of measuring different particle sizes in a sample, (up to 12 sizes), it is common practice to use just the total particle count as an indication of filter performance. Recent research in direct filtration has focused on the effect of influent properties of the suspension on the removal of such 
parameters as turbidity, color and particle count. The effect of the influent particle size distribution on the head loss, effluent quality, and effluent particle size distribution has not been studied.

\subsection{Objectives of the study}

The research reported in this study focuses on the physical aspects of coagulation, flocculation and filtration, specifically the changes in particle size distribution brought about by these processes. specifically, the objectives of this research are:

(1) to investigate the influence of physical and chemical parameters in the coagulation and flocculation processes on the particle size distribution of dilute suspensions.

(2) to investigate the influence of influent particle size distribution on granular media filtration in terms of head loss development across the media and effluent quality (particle count), and

(3) to investigate the relationship between the influent and effluent particle size distribution with different filtration media sizes. 


\section{LITERATURE REVIEṬ}

In this chapter, the three most important processes in water treatment will be reviewed. The three processes are the ones used in treating dilute suspensions, mainly those used in direct filtration. These processes include coagulation, flocculation and filtration.

Ther first process, coagulation, is one in which a hydrolyzing inorganic salt of aluminum or iron is dispersed by turbulent motion throughout the water to be treated. This process includes the addition of the coagulant and the rapid mixing of the suspension. It was suggested that the function of rapid mixing is to insure complete homogenous coagulation (Hudson et al. 1967). In the absence of sufficiently rapid mixing, part of the water would be overtreated with coagulant, while other parts are undertreated or not treated at all. Recently, it was shown that rapid mixing greatly influences the rate of subsequent flocculation during the slow-mix operation (Letterman et al. 1973. Amirtharajah 1978, Amirtharajah et al. 1982 and 1986 and collins et al. 1987).

The second process is flocculation which consists of the grouping and compacting of coagulated particles into larger aggregates called floc particles. The aggregation of particles is accomplished by gentle slow mixing for 20 to 30 
minutes. Mixing intensity is described in terms of the velocity gradient, $G$, which is derived from the mean amount of work applied per unit of time to a unit volume of fluid at a definite viscosity (Camp 1955).

The third process is filtration which consists of using single media (usually graded sand), dual-media (sand and anthracite) or multi-media (sand, anthracite, and garnet sand) as filtering materials. In conventional treatment, filtration is considered a polishing step but in direct filtration it is considered the most important operation unit of the entire treatment process.

The following sections describe the three processes in terms of the mechanisms involved and the traditional methods of optimization of these processes. The last section is a review of particle counting and the use of particle size distribution in the treatment of waters.

\subsection{Coagulation}

Coagulation is defined as the chemical destabilization that leads to a reduction of the potential energy of repulsion between particles (Tekippe et al. 1971 and o'Melia 1972). Coagulation includes the reduction of electrostatic charges between particles as well as the decrease in surface potential accomplished by the adsorption of counter ions onto the particle surfaces. 
Almost all suspended particles found in natural waters possess a charge (predominantly negative). The existence of these charges is due to one or more of the following reasons (O'Melia et al. 1964, O'Melia 1969, and 0!Connell 1978):

(1) an imperfection in a crystal lattice

(2) ionization of molecules at the particle surface

(3) direct chemical reactions with specific ions in solution (chemisorption)

(4) physical adsorption of ions from solution (an example is hydrogen bonding).

\section{Mechanisms of Coagulation}

The coagulation of particles in natural waters involves two distinct steps (stumm et al. 1968, 1972 and Dempsey et al. 1984b):

(1) Particle transport to bring about interparticle contacts, and

(2) particle destabilization to permit attachment when contact occurs.

Theories of particle transport are based upon fluid and particle mechanics; theories of particle destabilization are based on colloid and interfacial chemistry.

Particle transport in aqueous systems is a physical process. It is accomplished by such phenomena as Brownian diffusion, fluid motion, and sedimentation, and is controlled by such physical parameters as temperature, velocity gradient, and particle size. particle 
destabilization is clearly is controlled by both chemical and physical parameters.

There are at least four different mechanisms at which particles present in natural waters can be destabilized. They are (Dempsey et. al. 1984):

(1) double-layer compression;

(2) adsorption to produce charge neutralization;

(3) enmeshment in a precipitate; and

(4) adsorption to permit interparticle bridging.

Double-layer compression

The destabilization of particles by the compression of the double-layer is purely electrostatic; ions of similar charge to the primary charge of the colloid are repelled, and counter-ions are attracted. Coagulants that cause destabilization by compressing the double layer are sometimes termed indifferent electrolytes. The effects of these coagulants were observed by Hardy in 1900 and summarized in the schulze-Hardy rule. This rule states that the destabilization of a colloid by an indifferent electrolyte is brought about by ions of opposite charge to that of the colloid (counter-ions), and that the coagulation effectiveness of these ions increases markedly with charge. For example, aluminum and iron salts are several times as effective as sodium compounds for the coagulation of negative colloids. Destabilization by the schulze-Hardy double layer compression is not important in most 
fresh-water treatment processes.

Adsorption and charge neutralization

Charge neutralization is similar to the Schulze-Hardy double-layer compression in that the coagulant affects the electric potential that is exerted by the charged particles. In charge neutralization, the coagulant specifically reacts with the contaminant. When destabilization is brought about by charge neutralization, then a stoichiometry exists between the coagulant and contaminant, and restabilization of the particles can occur if the stoichiometric ratio is exceeded. Destabiliztion by charge neutralization is a very common process in water treatment.

Enmeshment in a precipitate

When salts of aluminum or iron are added to water in concentrations exceeding their solubility limit, they form an insoluble precipitate. This type of destabilization is known as the enmeshment mechanism or sweep floc (Packham 1965; Stum et al. 1968). The particles present in suspension are entrapped in the amorphous precipitate that is formed. This mechanism of destabilization predominates in water treatment applications where pH values are generally maintained between 6 and 8 and coagulants are used at concentrations exceeding saturation with respect to the amorphous metal hydroxide solid that is formed. 
Adsorption and interparticle bridging

Destabilization of particles by high molecular weight polymers is the result of bridging together two or more particles as was suggested by the chemical bridging theory developed by kane and co-workers (1963) and Black et al. (1965a). The chemical bridging theory proposes that a polymer molecule can attach itself at one end to the surface of the colloidal particle with the remainder of the molecule extending into the solution where it is attached to another particle forming a chemical bridge between the particles.

The effects of raw water turbidity on coagulation with aluminum or iron salts are illustrated schematically in Figure 2.1. The residual turbidity is plotted as a function of coagulant dosage at constant $\mathrm{pH}$ for natural waters containing different suspended particulate concentrations treated with aluminum or iron salts. The results are characterized by four zones. At low coagulant dosages (zone 1), insufficient coagulant has been added to destabilize the particles. Increasing the coagulant dosage induces destabilization (zone 2 ) and further increase in dosage can restabilize the particles (zone 3 ). In zone 4, a sufficient degree of oversaturation occurs to produce a rapid precipitation of aluminum or ferric hydroxide in which particles are removed by adsorption on or by enmeshment in these solids. Figure 2.1 is based on the discussions presented by O'Melia (1972), stumm et al. (1968), O'Melia et al. (1967a), and O'Melia (1978a). 


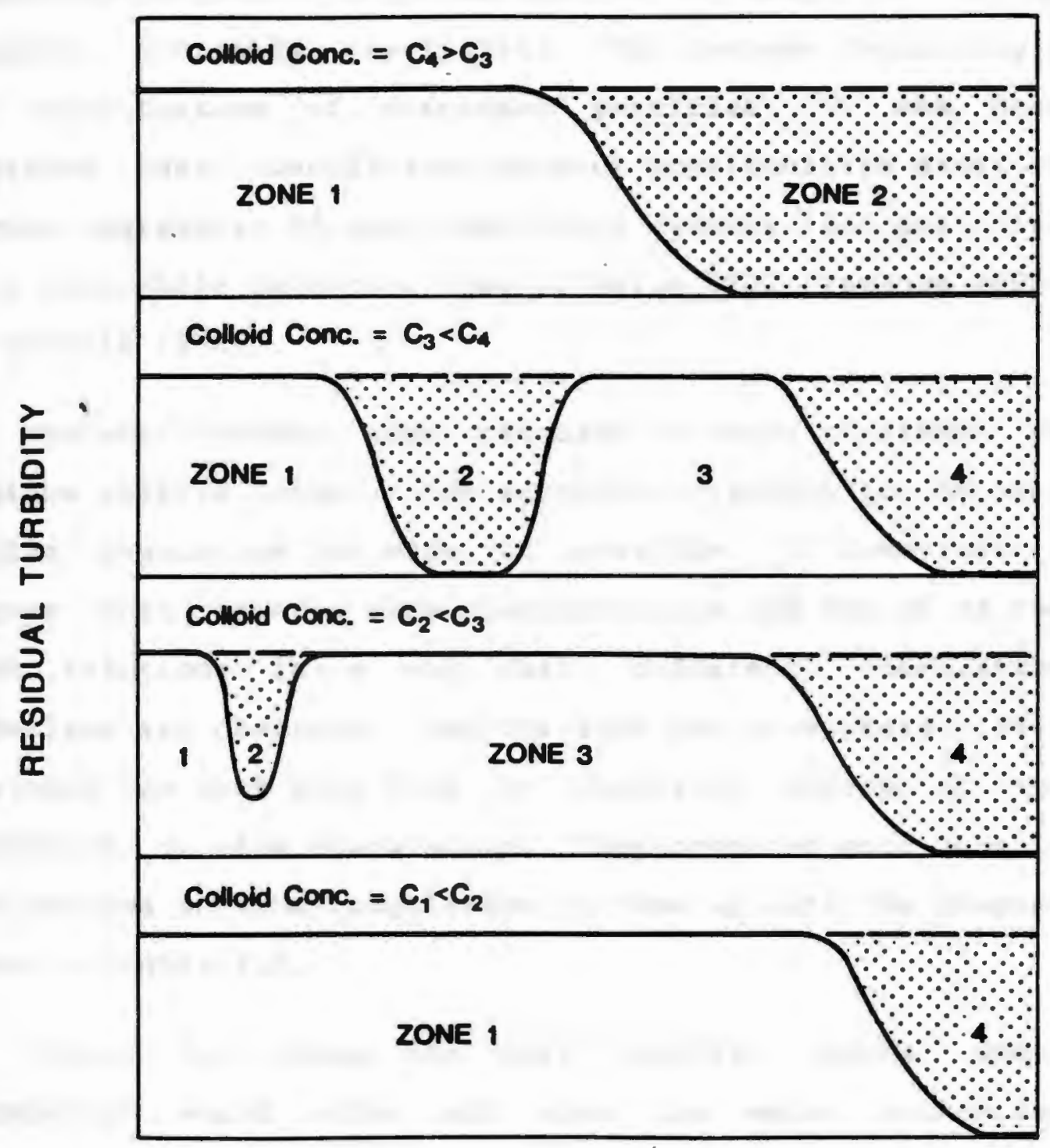

COAGULANT DOSAGE

Figure 2.1 schematic representation of the coagulation of colloidal suspensions by $A$ (III) or Fe(III) salts. 
For low turbidity waters ( $C 1$ in Figure 2.1), only one region for particle removal is observed. In this case, coagulation requires the production of a large excess of amorphous hydroxide precipitate. For systems containing a low concentration of suspended particles, it has been suggested that insufficient contact opportunities exist to produce aggregates of even completely destabilized particles in a reasonable detention time (0'Melia 1972, Packham 1965, and O'Melia 1978c).

The "most commonly used coagulant in water treatment is aluminum sulfate (alum). The extensive research in the area of alum coagulation has made it possible to construct a diagram that relates alum concentrations and the pH of the mixed solutions in a way that different coagulation mechanisms are observed. Amirtharajah and co-workers (1982) developed one such plot from an extensive review of the literature on alum coagulation. They combined more than 18 past studies on alum coagulation to come up with the diagram shown in Figure 2.2 .

Figure 2.2 shows the very specific areas where coagulation would occur and also the major mechanisms causing coagulation. Destabilization by charge neutralization mechanisms is seen to occur in the pH range of 5.25-6.40 with alum concentrations of less than $2 \mathrm{mg} / \mathrm{L}$. The area for optimum sweep coagulation-the area for best settling floc with the lowest coagulant dosage- is defined by an alum dose of 20 to $50 \mathrm{mg} / \mathrm{L}$ with a final pH of $6.8-8.2$. 


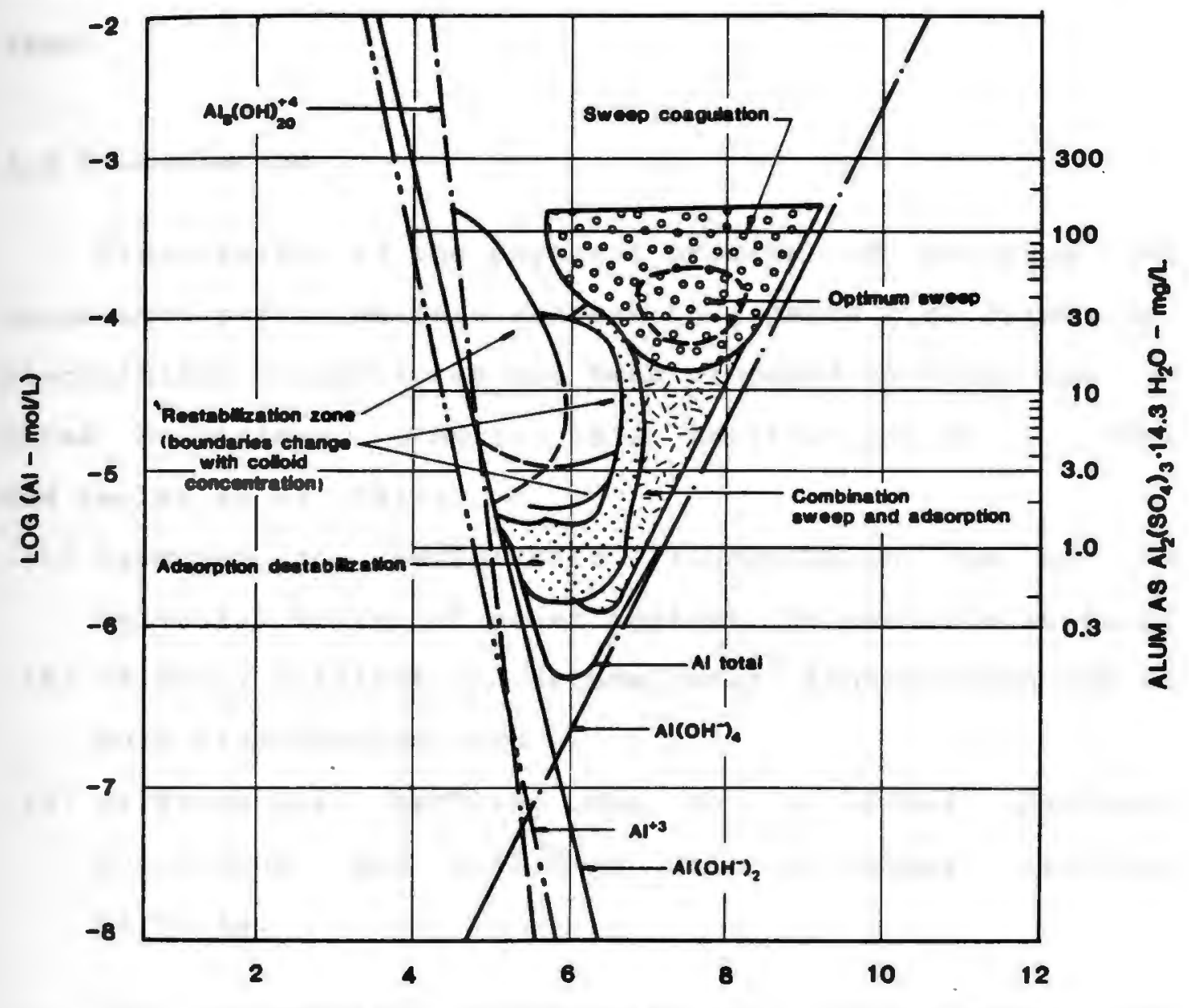

PH OF MIXED SOLUTION

Figure 2.2 Design and operation diagram for alum coagulation. (Source: Amirtharajah et al. 1982) 
Sweep coagulation is the most dominant region in the coagulation of particles. The restabilization phenomenon occurs mostly in the low pH range with $2 \mathrm{mg} / \mathrm{L}$ or more of alum.

\subsection{Flocculation}

Flocculation is the physical process of bringing the coagulated particles into contact to promote floc formation. Flocculation of particles has been proposed to occur due to three mechanisms (O'Melia 1972, Amirtharajah et al. 1986 and Lawler et al 1983):

(1) Brownian or perikinetic flocculation due to the molecular motion of water against the particle surface;

(2) velocity gradient or orthokinetic flocculation due to bulk fluid motion; and

(3) differential settling due to a larger particle overtaking and colliding with a slower settling particle.

The mathematical description of flocculation was developed by smoluchowski more than 70 years ago. The mathematical formulation was based on the considerations of the binary collisions between particles in a suspension. Basically the model describes the expected change in the number concentration of particles of any particular size. Smoluchowski's equation can be expressed as (Fair et al. 1964, Tekippe et al. 1971, and Lawler et al. 1983): 


$$
\frac{d N}{d t}=\frac{1}{2} \alpha \sum_{i+j=k} b(i, j) N_{i} N_{j}-\alpha N_{k} \sum_{i=1}^{c} b(i, k) N_{i}
$$

where

$$
\begin{aligned}
N= & \text { the number concentration of particles } \\
i, j, k= & \text { subscripts denoting particular particle size } \\
t= & \text { time } \\
c= & \text { the largest size considered } \\
\alpha= & \text { the collision efficiency factor, which is defined } \\
& \text { as the fraction of predicted collisions that result } \\
& \text { in attachment } \\
b(i, j)= & \text { the collision frequency function ( number of } \\
& \text { collisions per second), which depends on the } \\
& \text { mechanism of interparticle contact (i.e. Brownian, } \\
& \text { shear or differential sedimentation) }
\end{aligned}
$$

The equations for the collision frequency function for the three mechanisms are (TeKippe 1971, Boadway 1978, and Lawler et al 1983):

for Brownian motion (perikinetic flocculation)

$$
\left(b_{i j}\right)_{p e}=\frac{2}{3} \frac{K T}{\mu}\left(r_{i}+r_{j}\right)\left(\frac{1}{r_{i}}+\frac{1}{r_{j}}\right) N_{i} N_{j}
$$

for fluid shear (orthokinetic flocculation)

$$
\left(b_{i j}\right)_{o r}=\frac{4}{3} N_{i} N_{j}\left(r_{i}+r_{j}\right)^{3} G
$$

for differential sedimentation 


$$
\left(b_{i j}\right)_{s e}=\frac{2}{9} \pi \frac{g}{\mu}\left(p_{p}-p_{i}\right) N_{i} N_{j}\left(r_{i}+r_{j}\right)^{3}\left(r_{i}-r_{j}\right)
$$

where

$$
\begin{aligned}
& \mathbf{K}=\text { Boltzmann's constant } \\
& T=\text { absolute temperature } \\
& \mu=\text { fluid absolute viscosity } \\
& \mathbf{r}=\text { particle radius } \\
& \mathbf{G}=\text { 'mean velocity gradient } \\
& \mathbf{g}=\text { gravitational constant }
\end{aligned}
$$

$P_{p} P_{1}=$ densities of the particles and liquid

The relative importance of the transport mechanisms in flocculation is based on the size of particles present in suspension. Perikinetic flocculation predominates when particles present are less than 0.1 micrometer in size (Ives 1978b)and with larger particles, orthokinetic transport predominates. Flocculation by differential sedimentation can occur in flocculators even though particle sedimentation is prevented by high fluid velocity.

In water treatment practice, orthokinetic flocculation is the most predominant mechanism in the aggregation of destabilized particles. The aggregation of particles is usually achieved by gently mixing the suspension. The mixing intensity is measured by the mean velocity gradient. The use of the mean value is based on the fact that the mixing gradient varies with position within the mixing 
chamber and with time at any one point.

The mixing intensity can be calculated from the relationship developed by Camp (1955):

$$
G=\sqrt{\frac{P}{v v_{1}}}
$$

where

$$
\begin{aligned}
& P=\text { power input, } w(f t-1 b / s) ; \\
& v_{1}=\text { volume of water receiving the input, } m^{3}(c u f t) ; \text { and } \\
& v=\text { dynamic viscosity, Pa-sec }(1 b-s e c / s q f t)
\end{aligned}
$$

The power transmitted from the mixer blade to the water can be determined by two methods. The first of these methods is the use of a torque meter which is placed on the drive shaft of the mixer. Then

$$
P=T_{q} \omega
$$

where

$$
\begin{aligned}
T_{q} & =\text { the measured torque; and } \\
\omega & =\text { the speed of the rotation in radians per second } \\
& =2 \pi n, n=\text { rpm of the impeller }
\end{aligned}
$$

The second approach for determining the power transmitted is to multiply the drag force on the paddle blade by the velocity of the blade relative to the suspension. The drag force is the Bernoulli dynamic pressure $\rho_{1}\left(v_{b}-v_{1}\right)^{2} / 2$ multiplied by the area $A_{p}$ and the 
drag coefficient (Ives 1978b). Therefore the power is given by:

$$
P=\frac{1}{2} C_{d} A_{p} P_{1}\left(v_{b}-v_{1}\right)^{3}
$$

where

$$
\begin{aligned}
C_{d}= & \text { Drag coefficient } \\
A_{p}= & \text { area of the paddle blade normal to the direction of } \\
& \text { motion } \\
v_{b}= & \text { mean paddle velocity } \\
v_{1}= & \text { mean suspension velocity in the direction of the } \\
& \text { paddle motion. }
\end{aligned}
$$

In the study reported here, the first method was used in determining the velocity gradient.

During flocculation, flocs continue to grow by orthokinetic flocculation until they reach a limiting size that, according to several observers such as Camp (1955), Boadway (1978), Matsuo et al. (1981), Cleasby (1984) and others, appears to be inversely proportional to the velocity gradient. Boadway (1978) presented a relationship between the maximum size of floc that can be formed and the mixing intensity. The relationship has the following form:

$$
D_{u}=k_{1}\left(\frac{W}{G}\right)^{1 /\left(\gamma_{1}-\gamma_{2}\right)}
$$

where

$\mathrm{D}_{\mathrm{u}}$ = maximum size of particle 


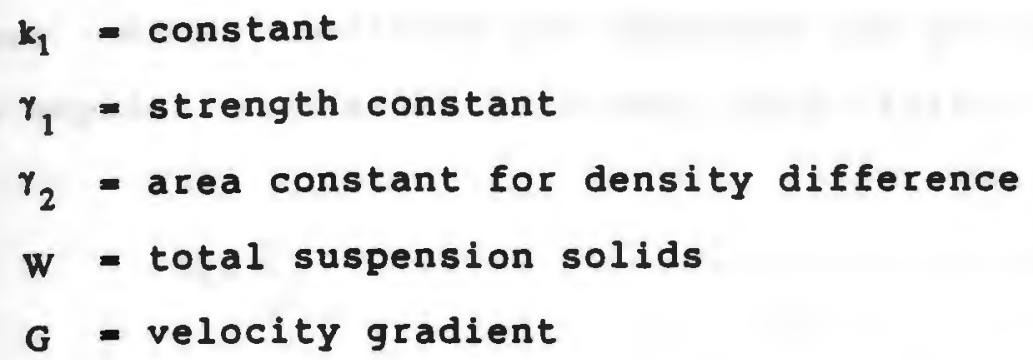

Floc breakup in agitated systems has been explained qualitatively by many researchers. The phenomenon of floc breakage is important in water treatment since it has a direct effect on the distribution of flocs in the suspension as was reported by previous experimental observations (Fair et al. 1964 and Ives et al 1973). When floc breakage occurs, then the downstream solid-liquid separation processes are affected. Floc breakup is the result of the interaction of individual flocs with fluid forces. A floc can be reviewed as an aggregate of primary particles that are bound together to form a larger particle that is extremely porous. The size and compactness of the floc and the strength of the bonds between the primary particles can all be expected to contribute to the ability of the floc to withstand shear by fluid forces.

Characterization of floc breakup has been proposed as the result of two different modes by Argaman et al. (1970) and Parker et al. (1972). The first mode is the erosion of primary particles from the exterior surface layers of the floc, and the second mode is the fracture of the floc to form a number of smaller floc aggregates. For both modes, an expression for the maximum stable floc size was derived 
above which the flocs are unstable and subject to breakup. The expression has the following form (Parker et al. 1972):

$$
D_{3}=\frac{C}{G^{f}}
$$

where

$D_{s}$ - maximum stable floc diameter

C = floc strength coefficient which depends on both floc and fluid properties

G -'velocity gradient

$f$ - exponent which depends on the breakup mode and size regime of eddies that cause disruption.

Equation 9 is similar to equation 8 which was developed for the maximum size of floc as a function of the velocity gradient.

\subsection{Optinization of Coagulation/Flocculation Processes}

The removal of particles from raw waters requires the optimization of the chemical and physical parameters involved in the solid-liquid separation processes. The main parameters that need to be optimized are:

(1) Coagulant dosage;

(2) coagulation $\mathrm{pH}$;

(3) mixing intensity; and

(4) mixing time. 
The basic laboratory tool used for determining the above parameters in water treatment operations is the jar test. A stirrer with flat paddles has traditionally been used as the impeller in jar testing. There has been a great deal of work to improve the jar test procedure by investigator's such as camp (1955, 1968), Harris et al. (1966), Tekippe et al. (1970), Argaman (1971), Vrale et al. (1971), Hudson (1973), Hudson et al. (1981) and cornwell (1983). Their work went beyond conducting the jar test by including an investigation of the processes of coagulation, rapid mixing, and flocculation and their effects on sedimentation and filtration. Some researchers such as Hudson modified the basic equipment by adapting square instead of the normally used rounded beakers. The above parameters are determined by minimizing turbidity or the remaining total particle count (Lai et al. 1975, Yeh et al. 1981, Reed et al. 1986).

With the use of the jar test procedure, there are many techniques that have been used as performance indicators. Some of the important techniques are:
(1) Turbidity measurement
(2) Settling velocity
(3) Speed of floc formation
(4) Filtrability number
(5) Conductivity
(6) Zeta potential
(7) Electronic particle counting. 
Turbidity measurement is the most commonly used technique as a performance indicator. This is because turbidity is easily measured with simple equipment that requires minimal operation. The turbidity measurement is based on the light-scattering technique which depends on such factors as particle shape, refractive index and the wave length of the light source (Black et al. 1965b, Beard et al. 1977, and Treweek 1979).

In the settling velocity technique, water samples are allowed to flocculate for a specific time, then allowed to settle and samples are taken at intervals from a fixed depth and analyzed for floc concentration. The results are used to compute the cumulative floc settling distribution and mean settling velocity which are used for assessing floc quality. In the speed of floc formation technique, the time between the coagulant addition and the first appearance of visible floc is recorded. It is a very simple technique but not very accurate since some kinds of floc are colorless.

The filtrability number technique was developed by Shull (1967) and consisted of a comparison of the time needed to filter a $200 \mathrm{ml}$ sample of jar test supernatant through a 0.45 micrometer membrane filter to the time required to filter an equal volume of distilled water under similar conditions. The ratio of these quantities has been defined as the filtrability number. 
The difference of conductivity before and after the addition of coagulant has been used as a technique for selection of the coagulant dosage in low alkalinity, colored waters. The only drawback of this technique is the fact that it is only applicable to waters that have a proper stoichiometric alkalinity-alum requirement ratio.

The zeta potential measurement technique involves measurement of the particle's electrophoretic mobility. This is accomplished by measuring the particle velocity in a suspension containing electrodes which establish an electrical field to motivate charged particles. The particle velocity is measured with a microscope. The technique is limited only when the coagulants being used are inorganic salts. According to stumm et al. (1968), the zeta potential technique is worthless when the coagulant used to destabilize the negatively charged particles is an anionic polymer.

Particle counting techniques have been used in the past 20 years by only a few investigators. Its limited use is mainly due to the cost and operation of the equipment. Techniques used in counting particles range from photographs, to microscopic counting of particles; to the recent development of electronic particle size analyzers. The use of particle counting in full scale treatment plants has been adopted as a supplement to the turbidimeter. This is because there are no specific criteria being set on the number of particles in the finished water as well as the 
nonexistence of a universal relationship between particle count and turbidity. Therefore, particle counting is used by researchers as a tool in understanding the actual changes that take place during the different treatment processes.

\subsection{Granular-Media Filtration}

Filtration is the second most important process in water treatment, the first being disinfection. Filtration is used in water treatment for the removal of suspended solids that are generated in the pretreatment process or are originally present in the raw water.

Filters are classified in different ways. There are slow and rapid sand filtration in which the flow of water through the media has a range of $0.04-0.12$ and 2-10 gallons per minute per square foot (gpm/sq.ft) for slow and rapid sand filtration, respectively. There are gravity and pressure filters in which the filter is open to the atmosphere or enclosed completely. Filters can be classified according to the direction of the water flow. There are downflow, upflow and biflow filters. Filters are also classified according to the media type. Single media filters are filters that consists of only one type of media such as sand. Dual-media filters are those that are made up of two different types of media with different specific gravities such as anthracite and sand. Multi-media filters are those that consist of three different types of media, usually anthracite, sand and garnet. Filters can also be 
classified according to flow control. There is constant and declining rate filtration in which the flow through the former is kept constant and declining through the latter. The most commonly used filter type in water treatment is a constant flow, single media, rapid, downflow, gravity filter.

Common filter sand specifications are: depth 24 to 30 inches, effective size 0.45 to $0.35 \mathrm{~mm}$, uniformity coefficient not greater than 1.65. Effective size is the diameter of the largest grain of sand in that 10 per cent of the sample, by weight, which contains the smallest grains. uniformity coefficient is the ratio of the largest grain in 60 per cent of the sample, by weight, which contains the smallest grains, to the effective size. The use of uniform sand has been limited in the United states due to the higher cost associated with screening the sand.

\subsubsection{Process Mechanisms}

Particle removal by a granular bed is the result of at least two different groups of mechanisms, acting independently of each other. First, the transport of suspended particles to the immediate vicinity of the solid-liquid interface presented by the filter (i.e. to a grain of the media or to another particle previously retained in the bed); and second, the attachment of particles to this surface $\left(0^{\prime}\right.$ Melia et al $1967 \mathrm{~b}$, yao et al 1971, Leclerc et al. 1972, Habibian et al. 1975, Ghosh et 
al. 1975, O'Melia 1985 and Huang et al. 1986). Particle transport is a physical process which is dependent on the mass transfer parameters. Particle attachment is basically a chemical process which depends on both physical and chemical parameters.

Transport step

The role of the transport mechanism is to bring the particles away from their streamlines, and into the vicinity of the grain or particle surface where the flow effect is minimum and attachment mechanisms are at a maximum. It is widely accepted that the flow within the media pores is laminar under the limits of the physical variables encountered in granular media filtration. The transport mechanisms are:

(1) Brownian and molecular diffusion

(2) Hydrodynamics

(3) Inertial impingement

(4) Interception and chance contact

(5) straining

(6) Sedimentation

The removal of particles by Brownian motion was found to be inversely proportional to the flow rate, and media size, and also to the square of the fluid viscosity (Yao et al. 1971). Theoretical as well as experimental results have shown that particles less than 1 micrometer in size are mostly removed by Brownian diffusion (Spielman et al. 1970, 
Cookson 1970, and Yao et al. 1971). The effect of Brownian motion in the typical filtration applications of water is insignificant, due to such parameters as suspended particle sizes and flow rates (Ives et al. 1965). Transport by molecular diffusion is also insignificant in water filtration mainly because of the size of particles present in the influent to the filter.

Transport by hydrodynamic mechanisms is due to the random motion of particles which is the result of the rotational and lateral drifts of particles. Since the particles are not spherical, then the shear forces acting on their boundaries are different, therefore the motion is random. Transport by hydrodynamic mechanisms is a function of the Reynolds Number.

Transport by inertial impingement is the predominant removal mechanism in fibrous filters used to clean air, but this mechanism is not of great significance in rapid sand filtration owing to the high viscosity of water. Inertial impingement is the result of the attachment of particles that have sufficient momentum to move downward, deviating from the original path around the sand grain, hence resulting in impingement on the sand grains.

Transport by the interception and chance mechanisms have been suggested to occur when the separating distance between the moving particle and the sand grain is less than or equal to half the diameter of the particle. The 
probability of the removal of a suspended particle was found to be proportional to the square of the grain diameter and inversely proportional to the third power of particle diameter (0'Melia et al. 1964). Yao et al. (1971), showed that the removal of particles due to interception is proportional to the square of the particle diameter divided by the media grain diameter.

Particle removal by the straining mechanism will always occur as long as there are particles too large to enter the pores of a filter, or large enough to span crevices within the pore structure. It was believed that filtration is a pure surface phenomenon and that straining at the entering face of the media should be as negligible as possible for successful operation lott et al.1970, and Sakthivadivel et al. 1972). Straining causes rapid head loss development due to the formation of a surface mat. This results in a shorter filter run.

Transport of particles by sedimentation is a function of the velocity of the fluid and the settling velocity of the suspended particle. Removals increase with the square of the suspended particle size and with the difference in density between the particle and the fluid lo'melia et al. 1964 and Yao et al. 1971). In water filtration, most of the suspended particles have a higher density than water and because the fluid velocity near the media grains approaches zero, therefore transport by sedimentation would be expected to occur. 
The transport of particles to the grain media can be expressed in term of the single collector efficiency of a media grain which is defined as the ratio of the rate at which suspended particles collide with a media grain to the rate at which they flow towards it. Considering only the transport by diffusion, interception and sedimentation, the total single collector efficiency, $\eta$ ' of a media grain is written as (Yao et al. 1971 and O'Melia 1985):

$$
\eta_{T}=\eta_{D}+\eta_{I}+\eta_{S}
$$

where

$\eta_{D}=$ single collector efficiency due to diffusion

$\eta_{1}$ = single collector efficiency due to interception

$\eta_{s}=$ single collector efficiency due to sedimentation. The single collector efficiency due to the above mechanisms can be expressed as:

$$
\begin{aligned}
& \eta_{D}=0.9\left(\frac{K T}{\mu d_{p} d_{s} v_{1}}\right)^{2 / 3} \\
& \eta_{I}=\frac{3}{2}\left(\frac{d_{p}}{d_{S}}\right)^{2} \\
& \eta_{S}=\frac{\left(p_{P}-p_{1}\right) g d_{p}^{2}}{18 \mu v_{1}}
\end{aligned}
$$

where

$K$ = Boltzmann's constant

$T$ = absolute temperature 
$\mu$ = water absolute viscosity

$d_{p}=$ particle diameter

$d_{s}=$ grain diameter

$v_{1}=$ filtration rate

$\rho_{p} P_{1}$ - densities of the suspended particles and water respectively

8 = gravitational constant

By plotting the single collector efficiency as a function of the particle size, Yao and co-workers (1971) were able to show theoretically and experimentally that there exists a size of the suspended particles for which the removal efficiency is a minimum. This critical suspended particle size is about 1 micrometer. For suspended particles larger than 1 micrometer, removal efficiency increases rapidly with particle size due to sedimentation and/or interception. For suspended particles smaller than 1 micrometer, removal efficiencies increase with decreasing particle size due to diffusion. The same observations were shown by Jordan et al. (1974), Ghosh et al. (1975), Habibian et al. (1975) and vigneswaran et al. (1985).

Attachment step

The attachment of suspended particles to the media grains is dependent on the chemical characteristics of the aqueous phase (such as $\mathrm{pH}$, type of ions and ionic strength) and the surface chemical properties of both the suspended particles and the filter media (such as surface charges and 
surface forces) (Loganathan et al. 1975). It has been shown that suspended particles several orders of magnitude smaller than the pore space are removed effectively by sand filtration. O'Melia et al. (1964) reviewed the various mechanisms of suspended particle removals and concluded that physical mechanisms alone could not adequately explain observed filter performance either in the laboratory or in the field.

The attachment mechanism is determined by surface forces between the filter grains and particles. These forces may result from the overlap of electrical double layers, from interaction of van der waals forces, or from other chemical effects (O'Melia et al. 1964 and Toregas $1983)$.

The mechanisms of attachment may be classified according to two models. The first is the "double-layer model" which is based on the interaction between the electrostatic repulsive forces and van der waal's forces. Marckle et al. (1961) were the first to propose the application of Van der Waal's intermolecular forces to water filtration. The authors contended that adhesion between suspended particles and grain media is solely due to attraction resulting from Van der waal's forces. The second is the "bridging model" which explains the attachment resulting from chemical bonding and bridging of the suspension particles through reaction with the flocculant. These effects were not treated by the first model. 


\subsubsection{Variables Affecting Direct Filtration}

Direct filtration is affected by a large number of physical and chemical variables. These variables can be divided into two categories. The first are those variables that are related to the filter itself. These include: depth of the media; (2) grain size diameter, shape and configuration; and (3) rate of filtration. The second category of variables are those that are related to the influent suspension. These include: (1) inflow particle concentration; (2) coagulant type and dose; (3) mixing conditions and detention times; and (4) $\mathrm{pH}$ and temperature of the suspension. The influence of these variables on the whole process is measured in terms of the head loss development and filter effluent quality.

Variables related to the filter

The depth of the media influences both the head loss and the filter effluent quality. As the depth increases. the head loss increases, but the effluent quality improves due to the increase in the holding capacity of the filter bed. The depth of media required to filter a given suspension, so that the maximum head loss is reached at the same time as the established limit on the effluent quality depends on all the variables mentioned before. There is no single formula that will give the required media depth when other variables are known, except extensive pilot plant experimentation. 
In terms of the sand size, particle removal and head loss are inversely proportional to the diameter of the media. The media size determines the degree of suspended particle penetration. Jung et al. (1974) showed that the penetration of a typical iron-floc is about 1 inch with 0.3 $\mathrm{mm}$ diameter sand and 19 inches with $1.00 \mathrm{~mm}$ sand under the same conditions of flow rate with 8 feet of head loss.

The effect of media shape on the process of direct filtration has not been investigated before. However, it has been' shown that for the same size of coal and sand, the former has a higher storage capacity due to the greater bed porosity. The porosity of a clean filter bed is normally 42-43 per cent for rounded sand and about 52-55 per cent for anthracite (Hudson 1969). The media shape is expected to influence the pore space and surface area of the media that is available for suspended particle attachment.

Filter bed configuration affects the effluent quality and head loss. Shea et al. (1971) concluded that fine media on top of coarse media (graded sand filters) is an inadequate configuration to produce long filter runs. The fine media on top prevents the penetration of suspended particles that could be removed in the bottom layers, therefore in depth filtration could not be achieved. Coarse on top of fine media configuration as in dual-media filtration can produce a better effluent quality and longer filter run lengths than single media filter configuration. 
High filtration rates (above $10 \mathrm{gpm} / \mathrm{sq} . \mathrm{ft.}$ ) have resulted in a poor water quality. An increase in the flow rate from 2 to $5 \mathrm{gpm} / \mathrm{sq} . \mathrm{ft}$. resulted in a floc penetration of 1 and 4 inches respectively for the same operational conditions as was presented by Jung et al. (1974). The volume of water that is produced when the flow rate is doubled is slightly higher than that at the low flow rate for the same head loss. It has been shown that doubling the flow rate results in a 45 per cent reduction in the filter run length. However when one considers the volume of water required for backwashing, low flow rates are more advantageous for both quality and volume output.

Variables related to the influent suspension

In direct filtration, the influent suspended particle concentration is a significant variable. High influent concentrations result in shorter filter runs due to the high solids loading from both the solids present in the raw water and the coagulant dosage needed for the destabilization of these particles. The use of coarse media with such high influent concentrations might increase the performance of the process. Low influent particle concentrations usually require a small size media to avoid fast breakthrough. For a clean filter, the rate of removal of particles with respect to depth is proportional to the concentration of particles in suspension (Iwasaki 1937's formulation). Low influent particle concentrations produce low head losses as 
well as low removal efficiencies. According to o'Melia et al. $(1978 b)$, this is due to the fact that in packed bed filters, the removal of particles depends on the number of retained particles which act as collectors. This indicates that for filters treating low turbidity waters, the depth of media in such filters should be deep as compared to filters that are receiving high suspended solids concentrations in which shallow depths are sufficient. However, there is no accepted criteria for the selection of the best media size and deptb for a particular water and experiments are always necessary.

The coagulant dosage required to destabilize the particles is proportional to the concentration of these particles. However, the type of coagulant affects the direct filtration process in terms of the head loss as well as the effluent quality. Adin et al. (1974) proposed that polymers are better than alum for use as a coagulant since lower polymer doses are required which produce strong and dense flocs with a low water content. The duration of the filter run is inversely proportional to the alum dose used. This is because filter clogging is related directly to the floc volume that is loaded onto the filter, and floc volume is related directly to the coagulant dose (Camp 1968, Shea et al. 1971, and wagner et al. 1982). There is no mention in the present literature about the relationship between the coagulant dose and the rate of head loss for the same raw water and media size. 
The influence of the mixing intensity on the effluent quality and head loss can be related to the largest flocs that can be formed and their distribution. Previous work indicated that high mixing intensities produce flocs that result in a deeper penetration into the filter bed than would occur from the flocs that are formed by low mixing intensities (Camp 1968; Hutchison 1976, and Collins et al. 1987). The floc penetration is a function of both the particle size distribution and the media size. With high mixing intensity there is an increased likelihood of filter breakthrough. Collins et al. (1987) observed that a mixing intensity of $110 \mathrm{~s}^{-1}$ resulted in a discrete pin-floc which penetrated the filter media to a greater depth (4-5 inches) than the 1 inch penetration observed at a mixing intensity of $60 \mathrm{~s}^{-1}$. The rate of head loss will be much lower at a high mixing intensity than at a lesser value due to the small floc volume produced as a result of mixing. Present literature does not show the effect of mixing intensity on the head loss development, instead the emphasis was placed on the quality of the filter effluent. There is no such thing as one optimum mixing intensity for all conditions but depending on the media size, configuration and depth, and raw water particle characteristics, there could be an optimum value. All previous workers in direct filtration have agreed on the fact that a high mixing intensity is better for the operation of such process when the media being used is single-graded in configuration. 
Unlike the mixing intensity, mixing time has an optimum value regardless of the media. The optimum mixing time results in the formation of the largest flocs and hence the larger floc volume. Since in direct filtration, sedimentation is omitted, then the optimum mixing time will result in the highest head loss development. Beyond the optimum mixing time, flocs start to break given smaller floc volume, hence deeper particle penetration, which could result in greater likelihood of particle breakthrough. The head loss development will of course not be as steep as with the optimum mixing time. In practice, the typical mixing time prior to sedimentation is 20 to 30 minutes depending on such factors as the water temperature, particle concentration, and mixing intensity (Hudson 1965, Kirchman et al. 1972, and Hutchison 1976). It is believed that the floc formed during the flocculation stage becomes progressively weaker when the mixing time exceeds the optimum. During flocculation, the flocs that are formed are subjected to constant collisions between themselves or the mechanical devices or both. Each partial or complete destruction of the floc particle during one of these collisions results in a floc which is being reformed and thus is weaker than its predecessor. Each successive action of reforming the floc particle weakens it even further. The present literature lacks any information on the influence of mixing time on the head loss development. The main concern was as mentioned before the finished water quality. However most of water treatment plants use head loss as the 
indicator of the filter run termination.

In section 2.6, the influence of influent suspension particle size distribution on the performance of granular media filtration will be presented.

The $\mathrm{pH}$ of the influent suspension influences both the effluent quality and head loss. Hutchison (1976) and Hudson et al. (1967) showed that the filter effluent turbidity rose as the $\mathrm{pH}$ of the flocculated water was increased above 7.00. The run time was also increased as the pH increased above the value of 7.00 .

\subsection{Particle size and Distribution}

Particle counting is a useful measurement technique permitting the quantification of suspended matter as well as providing a better understanding of the changes in particle size distribution in the water treatment processes. Basically, there are three methods that are commonly used in particle counting. These are:

(1) Manual via microscopic examination;

(2) electrical resistance; and

(3) light blockage.

Microscopic techniques provide a direct measurement of particulate material within the size constraints of the microscope. The microscopic technique produces some information on particle shape, number and type but it does not produce size distribution data. The major drawbacks of 
this technique are the fact that it is slow, difficult, and not amenable to automation.

Particle counting by the electrical resistance technique is based on the conductivity of the fluid. The technique is not widely used since it requires that the sample be mixed with a strong electrolyte (NaCl or NaOH) to increase specific conductance of the solution. The use of either electrolytes could distort the data because NaOH can precipitate calcium, and magnesium salts and NaCl may cause salting out of certain colloids (Tate et al. 1978).

Particle counting by light blockage is the most commonly used technique in the water treatment applications. The technique is based on the principle of light blockage. Particles in fluid suspensions flow through a channel past a window of known area which has a collimated light beam that shines through the fluid at right angles to the direction of flow. When a particle passes across the beam, it partially blocks the light falling on a photodiode. The pulse generated is proportional to the projected area of the particle, and particle size is specified in terms of equivalent spherical diameter. The most commonly used instrument is the BIAC Model PC-320. It is easy to operate and capable of providing reproducible results compared to other instruments. The resulting particle counts and sizes are comparable to those achieved by electronic particle counting and sizing (Treweek et al. 1977). 
Natural waters contain many particles of various sizes. The distribution of these particles can be described mathematically. This particle size distribution may be the most important physical characteristics of the system. It has been shown that the particulate size frequency distribution in natural waters follows closely the power-law distribution function (0'Melia 1978a, Kavanaugh et al. 1980, O'Melia 1980, Ramaley et al. 1981, Lawler et al. 1983, and wiesner et al 1987). The power-law is stated as:

$$
\frac{\Delta N}{\Delta L}=A L^{-\beta}
$$

where

$L=$ Particle size interval;

$N=$ number of particles of size $L$; and

$A \beta=$ are empirical constants.

Taking the logarithm of both sides of equation 14 gives :

$$
\log \frac{\Delta N}{\Delta L}=\log A-\beta \log L
$$

Equation 15 represents a straight line with $A$ as the intercept and $\beta$ as the negative slope. The $A$ value signifies the concentration of particles in suspension whereas the $\beta$ constant signifies the different size contribution to the total number of particles. The value of $\beta$ is an important indicator of the slope of the size 
distribution in the region over which it is valid. Values of $\beta$ for natural waters ranged from 1.80 to 4.50 as was reported by Kavanaugh et al. (1980). The exponent $\beta$ will be referred to as the distribution slope from now on.

Particle size distribution can be presented in three forms depending on the method used to describe particle size. Particle volume, surface area, or diameter may be used, resulting in the following particle size distribution functions (Ravanaugh et al. 1980):

$$
\begin{aligned}
& \frac{d V_{p}}{d(\log L)}=2.3 \frac{\pi}{6} A L^{(4-\beta)} \\
& \frac{d S}{d(\log L)}=2.3 \pi A L^{(3-\beta)} \\
& \frac{d N}{d(\log L)}=2.3 A L^{(1-\beta)}
\end{aligned}
$$

Particle size distribution in raw water can be shifted by physical and chemical means (Hannah.et al. 1967, Treweek 1979, and Ramaley et al. 1981). The shift in the particle size distribution can be measured by the distribution slope, .

The change in the distribution slope, $\beta$, with coagulant dosage in the coagulation process, has never been investigated before. The change in the particle distribution as the chemical dosage is increased could help in understanding the mechanisms of coagulation. Moreover, 
the influence of $\mathrm{pH}$ on the coagulation could be explained by a particle size distribution analysis. Previous researchers, such as Tanaka et al. (1986), have used the particle counting technique in optimizing the coagulant dose. This was done by plotting the remaining total number of particles at a specific time on the ordinant and the coagulant dosage on the abscissa. The optimum coagulant dosage is usually the one that corresponds to the lowest particle count. This technique does not indicate any thing about the distribution of particles which is the most influential parameter on the subsequent processes.

The influence of mixing intensity and time on the particle size distribution has not received great attention. The influence of these operational parameters on the particle size distribution can be measured by the distribution slope, $\beta$. Lawler et al. (1983) investigated the influence of mixing intensity and time on the particle size distribution for concentrated suspensions. The concentrations used in their study were 200 and $600 \mathrm{mg} / \mathrm{L}$ of colloidal silica. Their findings indicated that the distribution slope, $\beta$, changes as the mixing intensity and time changed. No previous investigation for dilute suspensions can be found in the literature. Treweek (1979) investigated the influence of mixing intensity on the particle size distribution in the flocculation and filtration of dilute suspensions using a batch scale set-up. The results were shown in plots of the number of particles 
against the particle size for raw water, coagulated-flocculated water, and filtered water. There was no attempt to use the distribution slope, $\beta$, as a performance indicator. The purpose of the study was to investigate the optimum mixing time prior to direct filtration using particle size analysis. By studying the changes in the particle size distribution resulting from the changes in the mixing intensity and time, it could be easier to understand the influence of these parameters on the filtration process in direct filtration.

The particle size distribution in the filter effluent of full scale or even pilot scale treatment plants has never been investigated in full detail as a function of the influent particle size distribution. Traditionally, the effluent quality might have been reported in terms of the total particle count with reference to the size range of measurements. Some treatments plants ( Monscvitz et al. 1983) use 20 particles/ml as the maximum in the effluent of the filter, others use 8 particles/ml (Tate et al. 1977) and some use 50 particles/ml (Tate et al. 1978). In the Providence water Treatment Plant, the average effluent particle count during six months of study was in the range of 10 to 20 particles/ml in the size range of 2-150 micrometers. 
2.6 Influence of the Influent Particle Size Distribution on the Performance of Granular Media Filtration.

This section deals with the influence of the influent suspension particle size distribution on the performance of granular media filtration, specifically the effluent quality and particle size distribution, and head loss development. since no previous comprehensive work was preformed in this area, then the discussion will be based on the available related literature as well as the author's own hypothesis. The discussion will be focused mainly on the influence of the pretreatment coagulant dosage, mixing intensity and time on the performance of granular media filtration.

There are many empirical parameters that have been adapted to measure the interaction between the suspended particles and the filter media. This is because the variables affecting particle transport and attachment are numerous and solid clarification is difficult. The first of these empirical parameters is the filter coefficient developed by Iwasaki in 1937. This coefficient is nothing more than a proportionality constant relating the removal of solids to the filter depth. Other parameters that have been used in the past are the bulking factor (which is related to the porosity of the deposited solids), the Filterability Number developed by Ives (1978a) and the specific deposit. The Filterability Number is a dimensionless parameter that relates the filterability of a suspension to filter material taking into account clarification, clogging and flow rate. 
The specific deposit is the volume of retained particles in a unit of filtering material.

As was mentioned before, the particle removal is inversely proportional to the diameter of the media, and regardless of the media size, there are always particles in the effluent which pass through or are dislodged from the filter. With fine media, these particles are in the small size range. Therefore, it would be expected that the particle size distribution will be sensitive to a slight change in the number of particles at any size within that range, hence the distribution slope, $\beta$, will be more scattered as would be the case when coarser media is used.

In cases where the filter run is controlled by particle breakthrough, the distribution slope, $\beta$, is expected to decrease with time because larger particles will start to penetrate the bed. This has been shown to occur in an actual treatment plant (Monscvitz et al. 1983) but no pilot plant studies have been performed to show the relationship between the $\beta$ value and the filtration variables. Most of the research in this area was performed using only one size of particles therefore no distribution of particles in the influent or effluent can exist.

For a clean bed, the head loss is a function of the media size, shape, depth and porosity as well as the flow rate and water temperature. When filtering a suspension that contains suspended solids, the head loss across the bed 
becomes also dependent on the physical and chemical characteristics of the particles. If the suspended particles are more completely removed by the media grains, then the head loss develops more quickly. when the distribution of particles are such that they penetrate deeper into the whole depth of the media, then the head loss develops more slowly with an even distribution across the bed.

The relationship between head loss and operation time gives an indication of the type of solids removal. Cleasby $(1969 b)$, concluded that the presence of an exponential head loss relationship with time is an indication of a surface removal mechanism whereas a linear relationship indicates in-depth filtration.

\subsubsection{Influence of Coagulant Dosage}

As the coagulant dosage is increased, more particles are destablized and agglomorated into larger particles. Since there will be an increase in the number of larger sized particles, the distribution slope, will be shifted to a lower value. Assuming no restabilization of the particles will occur, at least at the concentrations used in this study, then the effluent quality is expected to improve as the coagulant dosage is increased. However, due to the rapid filling of the pores within the bed resulting from the increase in the influent solids, the total volume of water produced will decrease dramatically and the effluent quality 
will also deteriorate. Therefore, the use of high coagulant dosage would result in low effluent quality as well as less volume production as compared to other dosages.

The deterioration of the effluent quality could be the result of the increase in the flow velocity within the bed as the pores fill since the flow rate is kept constant during the run. Therefore, some of the materials that have been previously retained by the media will appear in the effluent as the flow rate is increased. Many researchers (cleasby et al. 1962, Cleasby et al. 1963, Cleasby 1969a, Bernardo et al. 1980, and cleasby et al. 1980) have found that declining rate filtration produces a better water quality than the constant rate filtration for the same reason mentioned above. Therefore, the effluent deterioration will be a function of the coagulant dosage and the media size. It would be expected that use of the fine sand for the filter media will result in a more rapid effluent deterioration than when using a coarser sand for the same influent quality. Also, it could be that the available surface area of the media is exhausted and the particles can not find sites to attach to and therefore simply pass through the media. Since the media surface area is constant, then it would be expected that the deterioration of the effluent quality would be much faster when the coagulant dosage is low. This is because the smaller suspended particles have a greater total surface area. Toregas (1983) concluded that in his experiments a 
decline in filtration efficiency appears to occur when about $35 \%$ of the available surface area of the media is covered with particles.

The effluent distribution slope, $\beta$, is expected to follow the same trend as that of the influent. As the coagulant dose increases, the slope of the distribution function will decrease for both the influent and the effluent. However, when the coagulant dose is increased to a level that causes a rapid clogging of the bed then the particles that are released from the bed will cause the effluent distribution slope to deviate from this pattern. The direction of the deviation is not known. Perhaps the magnitude of the distribution slope will give an idea of the type of particles that have the tendency to be detached from the sand grains.

An increase in the coagulant dosage results in an increase in the solids produced from a given raw water. Therefore, the rate of head loss will increase with the increase in the chemical dosage. The relationship between the chemical dosage and the filter run length has been shown to be of the exponential type (shea et al. 1971, Hutchison et al. 1974, Hutchison 1976 and Foley 1980). In terms of the distribution slope, $\beta$, the higher the coagulant dosage the larger the flocs, hence the lower the $\beta$ value. As the size of the flocs increases, the chance of non-floc particle penetration is reduced and suspended solids penetration of the filter is minimized. This effect is more dominant with 
the fine to medium sand.

\subsubsection{Influence of Mixing Intensity}

The mixing intensity has a great influence on the particle size distribution of raw waters. High mixing intensities produce flocs that are small in size resulting in high distribution slope values. When the mixing intensity is low, the distribution of the flocculated particles is slanted towards the larger sizes and hence less filter penetration is expected. Most of the flocs will deposit on the surface and they will aid in the removal of the smaller particles. However, it is expected that most of the effluent particles will be in the small size range since deep penetration is not expected at low mixing intensities.

The effluent distribution slope, $\beta$, is expected to be inversely proportional to the mixing intensity. At a low mixing intensity the remaining small particles will go through the bed and show up in the effluent resulting in a high value for the effluent distribution slope. When the mixing intensity is high, small particles will be produced in the flocculator and more particles with a wide range of sizes will show up in the effluent resulting in a low effluent distribution slope. It should be noted that the media size plays an important role in the effluent distribution slope and the above hypothesis is a generalized one. Perhaps there is an optimum mixing intensity for different media sizes that will give the best particle 
removal.

The increase in the mixing intensity results in a smaller floc formation hence larger distribution slope values. Depending on the media size, a low mixing intensity causes a rapid head loss development mostly in the top layer of the sand media. A high mixing intensity on the other hand results in the formation of pin-point flocs that tend to penetrate deeper causing less head loss development and a more even headloss distribution across the depth of the media.

\subsubsection{Influence of Hixing Time}

Optimum mixing time causes only the small particles to show up in the effluent since most of the particles are deposited on top of the media. It should be pointed out that particles that are produced by high mixing intensity are different than the ones that are produced by high mixing time even if they both have the same distribution. The difference between the two is the shape of the particles. It is expected that the high mixing times produce fractions of the actual particles whereas high mixing intensity produces whole small particles. Therefore, the degree of penetration and arrangement of the particles within the bed are quite different and the effect on the effluent quality and head loss also will be different. 
The effluent quality in terms of the total particle count should improve with an increase in the mixing time beyond the optimum value. This is because smaller particles penetrate deeper in the sand bed and the removal of subsequent particles would be improved due to particle-particle removal mechanism. The optimum mixing time causes large flocs to be deposited on top of the sand bed and hence smaller particles will pass and show up in the effluent. This phenomenon should be more apparent using fine media as compared to coarser media. Also because of the rapid clogging of the sand bed, it is possible that some of the attached particles will be detached by the high flow through the pores causing an increase in the total particle count in the effluent water. Bed clogging is considered either surface or pores of the media depending on the media size.

The effluent distribution slope with different mixing times is expected to follow the same pattern as that with the mixing intensity. Optimum mixing time produces large flocs that cause small particles to pass through the bed therefore the effluent distribution slope values are expected to be high. As the mixing time increases flocs start to break giving rise to smaller particles and hence deeper filtration penetration is expected. The effluent distribution slope values then are expected to decrease with the increase in the mixing time. 
Since the optimum mixing time gives the largest floc formation, then its application would only be appropriate for those processes that employ sedimentation before filtration, i.e. conventional drinking water treatment. In contact or direct filtration all the solids that are produced are deposited in the filter, therefore external sedimentation is omitted.

The optimum mixing time is not necessarily the optimum for filtration but it is for sedimentation. The optimum mixing time produces flocs that are capable of gravity settling. However, in direct filtration since there is no pre-sedimentation, the flocs are not settled out but are instead removed by the filter.

The increase in the flocculation mixing time results in an increase in the distribution slope hence a deeper particle penetration. Therefore, as the mixing time increases the head loss development across the bed is expected to decrease. However, there exists a mixing time after which the rate of head loss will not change. This is because an equilibrium is reached where no more breakage occurs.

The influence of the pretreatment parameters on the performance of granular media filtration can be summarized in Table 2.1 . 
Table 2.1- Influence of the pretreatment parameters on the performance of granular media filtration.

Pretreatment

$\begin{array}{ccccc}\text { parameter } & \text { Influent } & \text { Effluent } & \text { Effluent } & \text { Head loss } \\ \text { (increase in) } & \beta & \text { quality } & \beta\end{array}$

\begin{tabular}{lllll}
\hline Coagulant dose & decrease & increase decrease & increase \\
Mixing intensity & increase & decrease decrease decrease \\
Mixing time & increase & increase decrease decrease \\
\hline
\end{tabular}




\section{EXPERIMENTAL INVESTIGATION}

The purpose of the experimental work was to investigate the influence of different operational parameters on the particle size distribution of raw water. The experimental part of the study consisted of three phases. The first phase was to investigate the influence of coagulant dose, $\mathrm{pH}$, mixing intensity and time on the particle size distribution in a conventional jar testing setup. The second experimental phase was to investigate the influence of mixing intensity on the particle size distribution using a larger pilot scale reactor. The pilot scale reactor was then used in the third experimental phase to investigate the influence of the influent particle size distribution on granular-media filtration in terms of head loss, and effluent quality and particle size distribution. This chapter details the equipment and materials that were used as well as the experimental procedure.

\subsection{Materials}

Jar test apparatus

The basic laboratory apparatus used for evaluating the coagulant dosage and type, mixing intensity and time, and pH is the Phipps-Bird jar test apparatus. The apparatus consists of a motor that provides mixing to 6 stirrers that 
have flat paddles. A 1-Liter circular glass beaker is used in conjunction with each stirrer. The maximum speed of paddles is $100 \mathrm{rpm}$. The equipment is manufactured by Phipps and Bird, Inc. of Richmond, VA.

Torque meter

Measurements of the torque transmitted to the pilot-scale reactor vessel were made using a Bex-ometer torque meter Model 38 manufactured by Bex Co. of San Francisco; CA. The range of measurement with this torque meter was from $0-125$ in-oz in 5 in-oz intervals. The accuracy of the torque meter is $\pm 5 \%$. Values of the shaft rpm were measured using a Gen Rad Model 1546 stropotac Digital stroboscope manufactured by General Radio Co., Concord, MA. The range of rpm measurement was 100-25,000 rpm.

pB meter

Measurements of water pH were made using a Fisher Accumet Model $142 \mathrm{pH}$ meter manufactured by Fisher scientific Co., Pittsburgh, PA. The meter was calibrated using pH 4.0, 7.0 and 10.0 standards before each use or once a day during pilot plant continuous operation. 
Particle size analyzer

The particle counter used to determine the number of particles in the water samples was a HIAC Model PC-320 particle size analyzer manufactured by Pacific scientific Co. of Montclair, CA. The particle size analyzer was equipped with an automatic bottle sampler and a 2-150 micrometer sensor. The automatic bottle sampler permitted the sampling of up to $130 \mathrm{ml}$ of suspension.

The results of the particle count were displayed in 12 separate channels. The size range in micrometers in each of these twelve channels was as follows: 2-5, 5-10, 10-15, 15-20, 20-25, 25-30, 30-35, 35-40, 40-50, 50-75, 75-100 and 100-150. The results were displayed in either delta or total count mode. The delta count mode would show only the number of particles within that interval, whereas, the total count mode displayed all the particles that have a size greater than or equal to the lower limit of that interval. The instrument was calibrated at the factory by using monosized spheres with known sizes.

\section{Particles}

The particles selected for use in this study were a colloidal clay product obtained from Pennsylvania Glass Sand Corp., Pittsburgh, PA. The particle characterization of this material is shown in Table 3.1. The selection of this type of particle was based on a comparison between the 
Table 3.1- MIN-U-Gel 200 Particle Characterization*

Typical Chemical Analysis

wt. $\%$

silicon dioxide

$\left(\mathrm{SiO}_{2}\right)$

66.21

Aluminum oxide

$\left(A I_{2} O_{3}\right)$

11.71

Magnesium oxide

( $\mathrm{MgO})$

9.70

Iron oxide

$\left(\mathrm{Fe}_{2} \mathrm{O}_{3}\right)$

4.02

Calcium oxide

( $\mathrm{CaO})$

2.92

Potassium oxide

$\left(\mathrm{x}_{2} \mathrm{O}\right)$

1.07

Phosphorous

$\left(\mathrm{P}_{2} \mathrm{O}_{5}\right)$

0.99

Titanium dioxide

$\left(\mathrm{TiO}_{2}\right)$

0.53

sulfur

$\left(\mathrm{SO}_{4}\right)$

0.25

carbon

$\left(\mathrm{CO}_{2}\right)$

2.57

0.03

Trace elements

Typical Physical Analysis

Specific gravity

2.40

pa

9.00

Packed bulk density, $1 b / f t^{3}$

50

Free moisture, \&

14

Average particle size, microns

5.50

Color

gray

* The above data were provided by the Pennsylvania Glass Sand Corporation, Quincy, Florida. 
available products used in previous studies documented in the literature and the particle size distribution of natural raw waters. Figure 3.1 shows the particle size distribution of the MIN-U-GEL 200 clay with two different concentrations, and the particle size distribution of the scituate Reservoir water. Figure 3.2 compares the particle size distribution of Scituate Reservoir water to MIN-U-GEL 200 and MIN-U-SIL 30, another particle product. Therefore, MIN-U-GEL 200 was selected because of the close physical similarities with that of natural waters.

\section{Chenicals}

The chemicals that were used were aluminum sulfate (alum), sodium hydroxide, and calcium hydroxide (lime). All three chemicals were analytical grade products of Fisher scientific co.

\subsection{Pilot-plant apparatus}

The pilot plant setup consisted of two parts. The first was the pretreatment apparatus which consisted of chemical and mixing tanks, pumps and mixers. The second was the granular media filtration apparatus which consisted of sand columns, flow meters, and a piezometer board. Figure 3.3 is a schematic diagram of the whole pilot plant setup. 


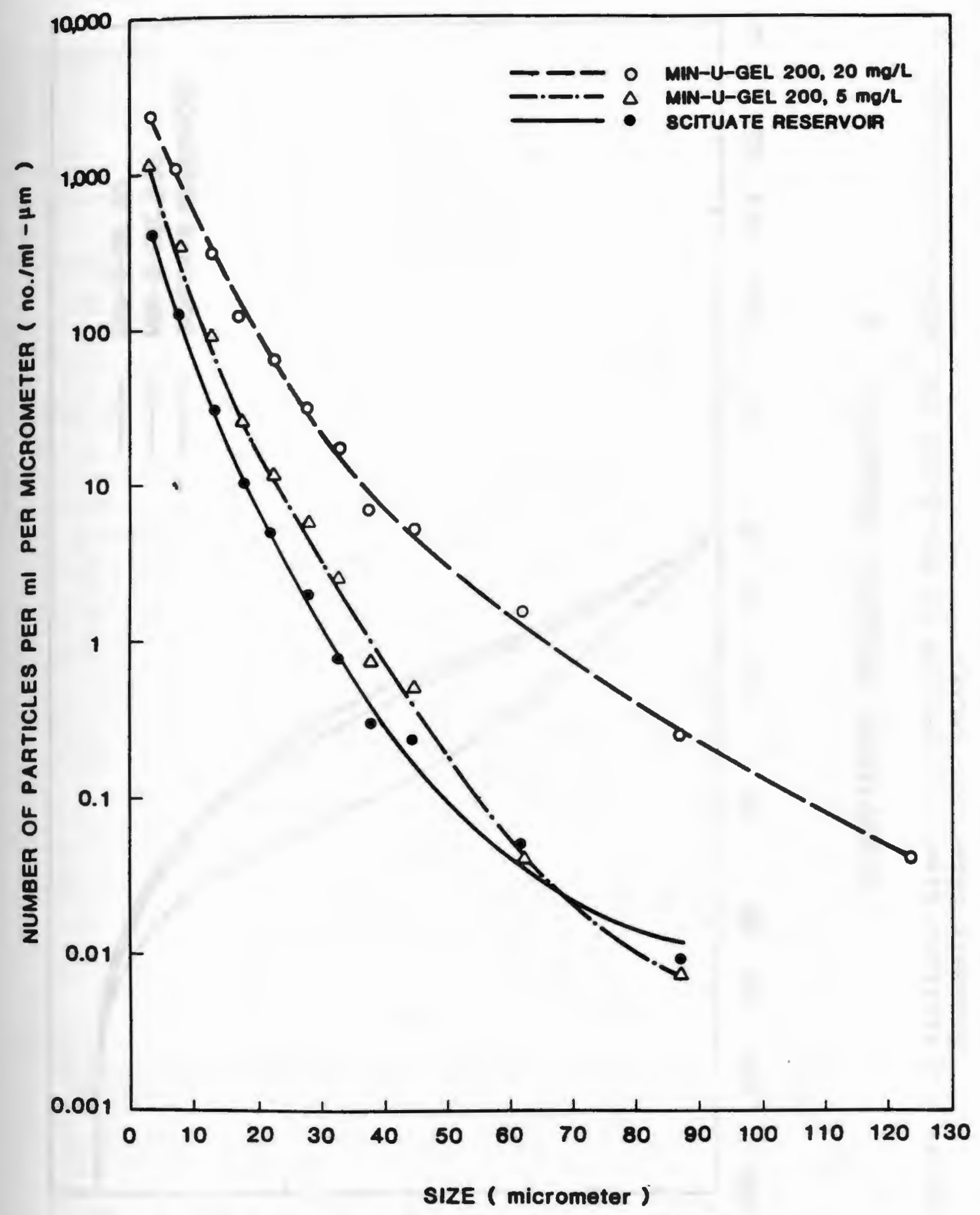

Figure 3.1 Comparison of the particle size distribution of the Scituate Reservoir water with MIN-U-GEL 200 at two different mass concentrations. 


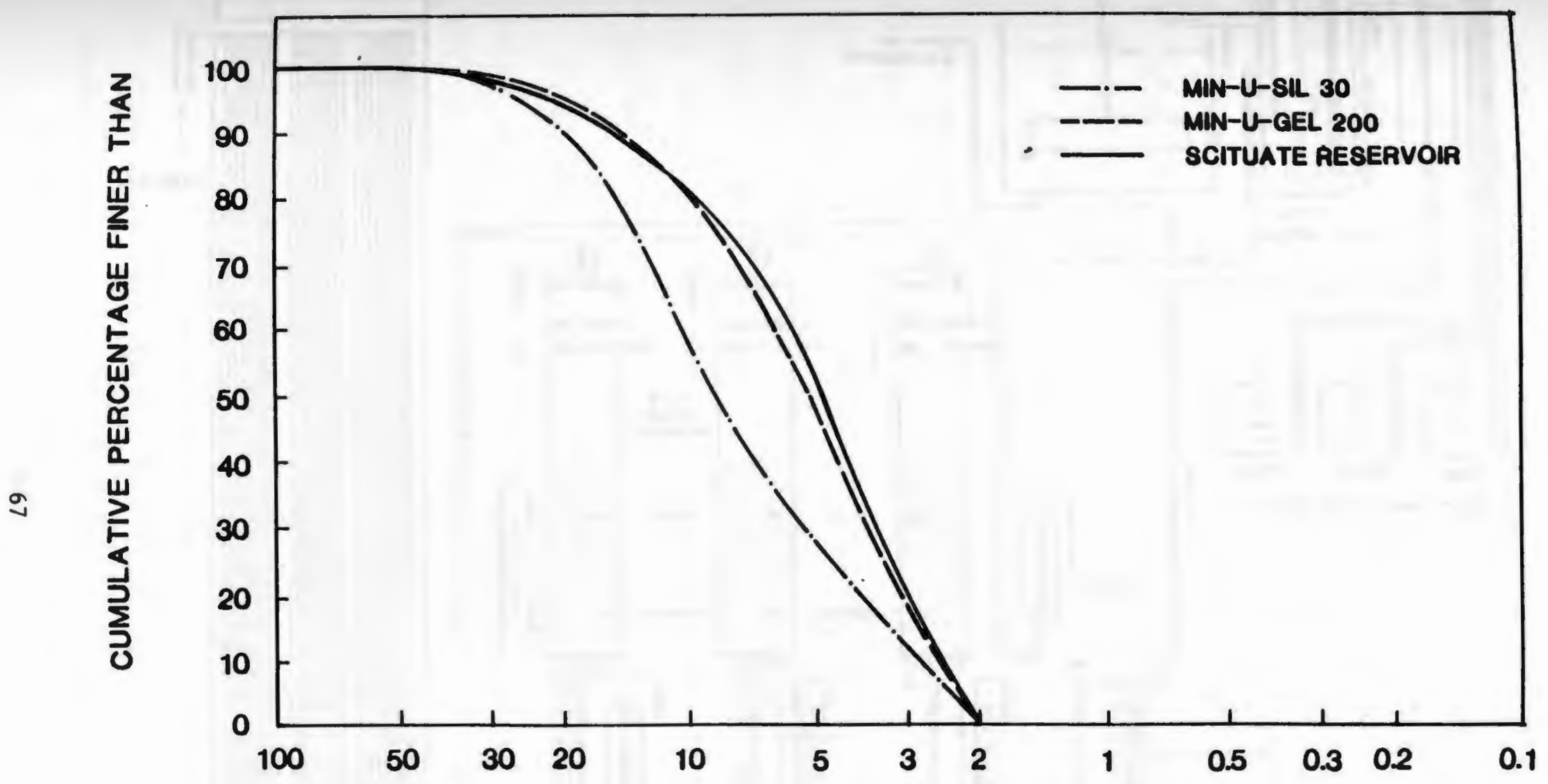

EQUIVALENT SPHERICAL DIAMETER, $\mu \mathrm{m}$

Figure 3.2 Particle size distribution of MIN-U-GEL 200, MIN-U-SIL 30 and scituate Reservoir water. 


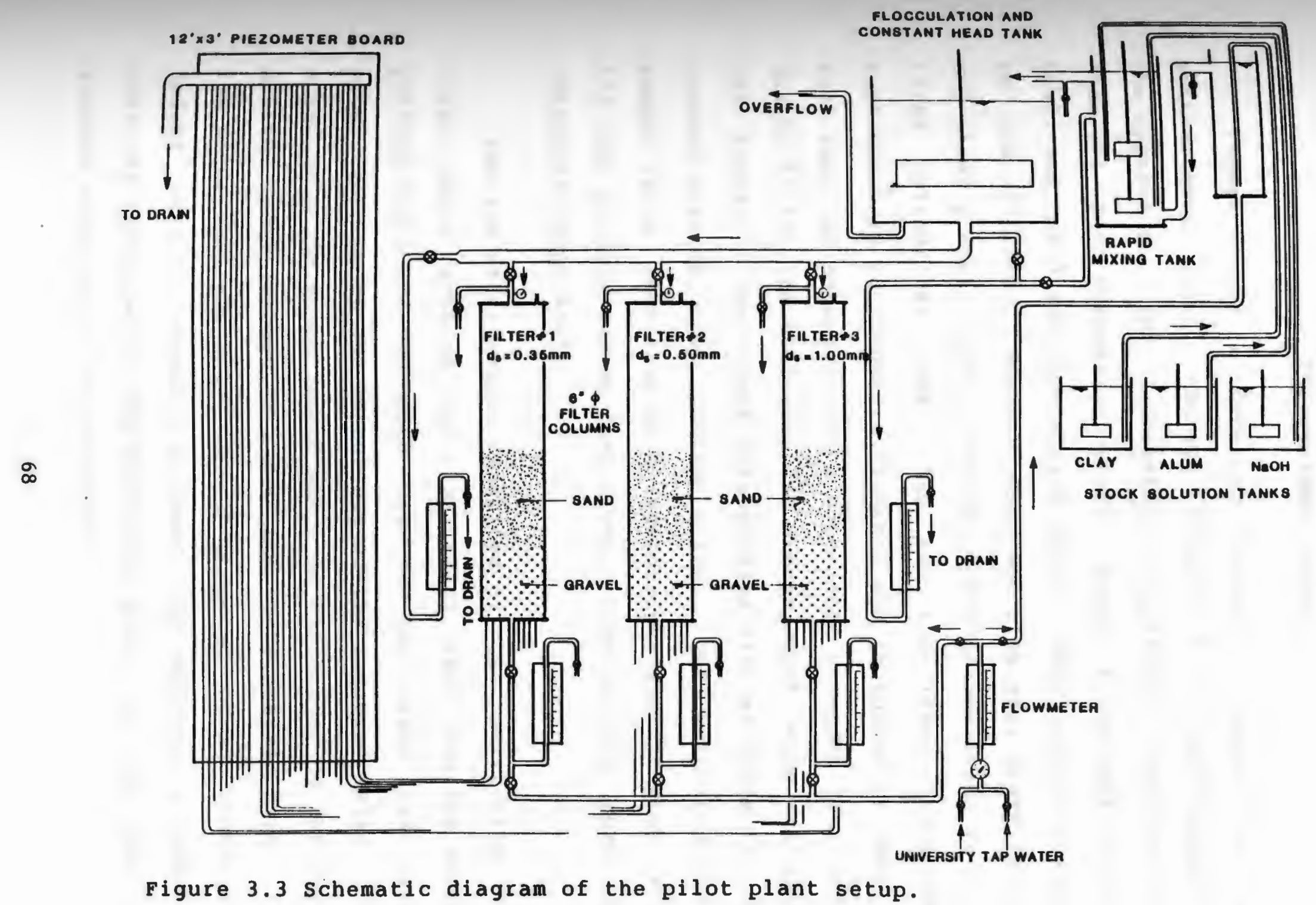




\section{Pretreatment setup}

Figure 3.4 is a detailed schematic diagram of the pretreatment setup, whereas Figure 3.5 is a photograph of the apparatus. The pretreatment apparatus consisted of chemical and suspension tanks, pumps, a raw water mixing tank, and rapid and slow mixing units. They were arranged in the following manner. Clay and alum were mixed in two 100 liter plastic tanks. Sodium hydroxide was mixed in a 50 liter cylindrical tank. The two 100 liter tanks were approximately 23 inches in diameter and 18 inches in height. Each tank was equipped with a mixer that provided continuous mixing at $100 \mathrm{rpm}$ utilizing two blades which each measured $2 \times 5$ inches. The tanks were covered with aluminum foil to prevent outside dust contamination. The solutions were pumped from each tank by a separate peristaltic pump using $1 / 4$ inch plastic tubing feed lines. Flow in each pump was controlled separately.

The raw water source consisted of the university tap water which entered the raw water tank from the bottom portion via a $1 / 2$ inch pipe where the tap water was mixed with the clay by the turbulence of the incoming water. The mixed solution moved upward where it discharged to the rapid mixing unit through a $11 / 2$ inch rigid plastic pipe. The raw water tank was $51 / 2$ inch in diameter and 17 inches in height with a capacity of about 1.59 gallons. A sampling port was installed at the discharge pipe of the tank to provide sampling of the raw water. 


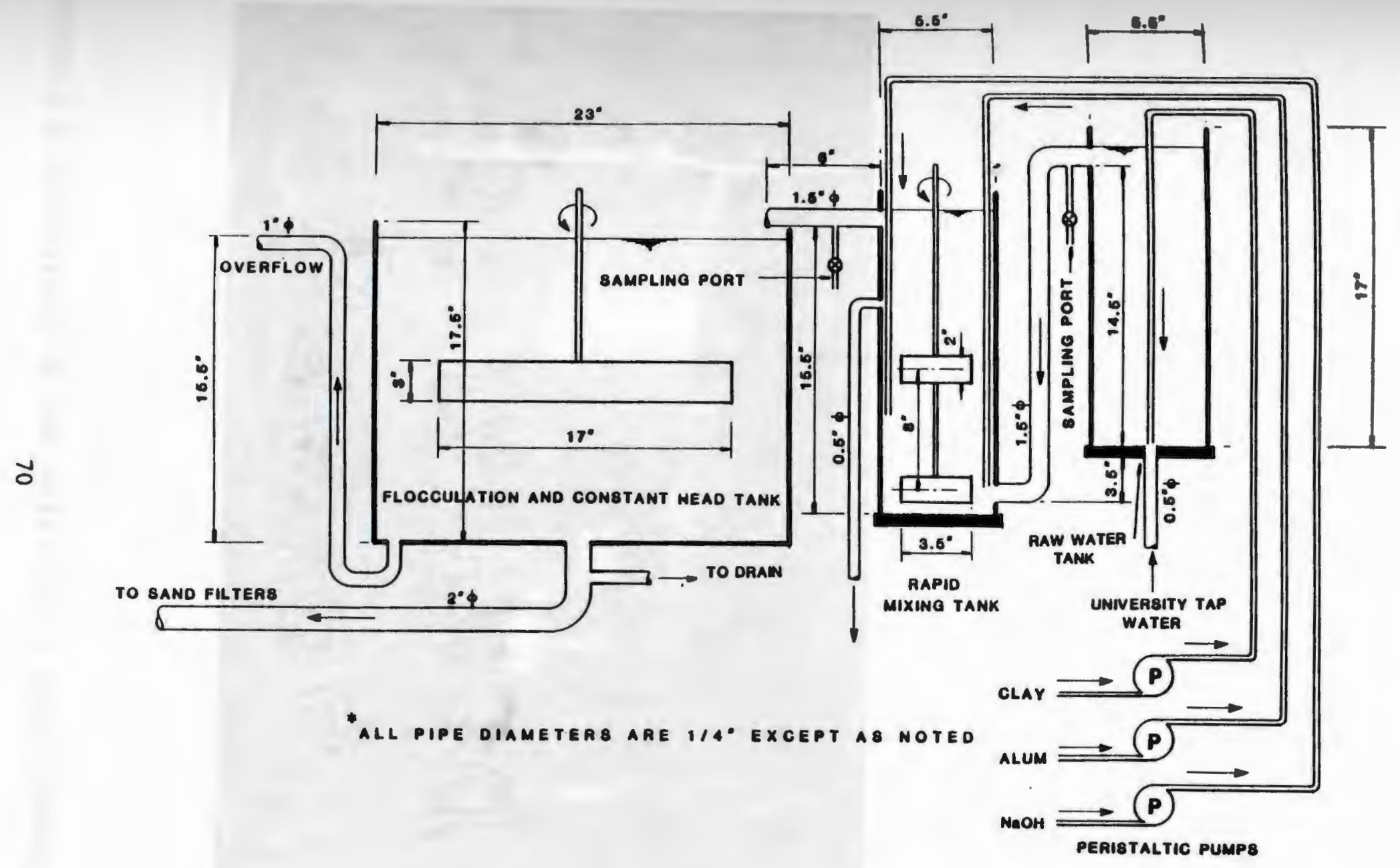

Figure 3.4 Detailed schematic diagram of the pilot plant pretreatment setup. 


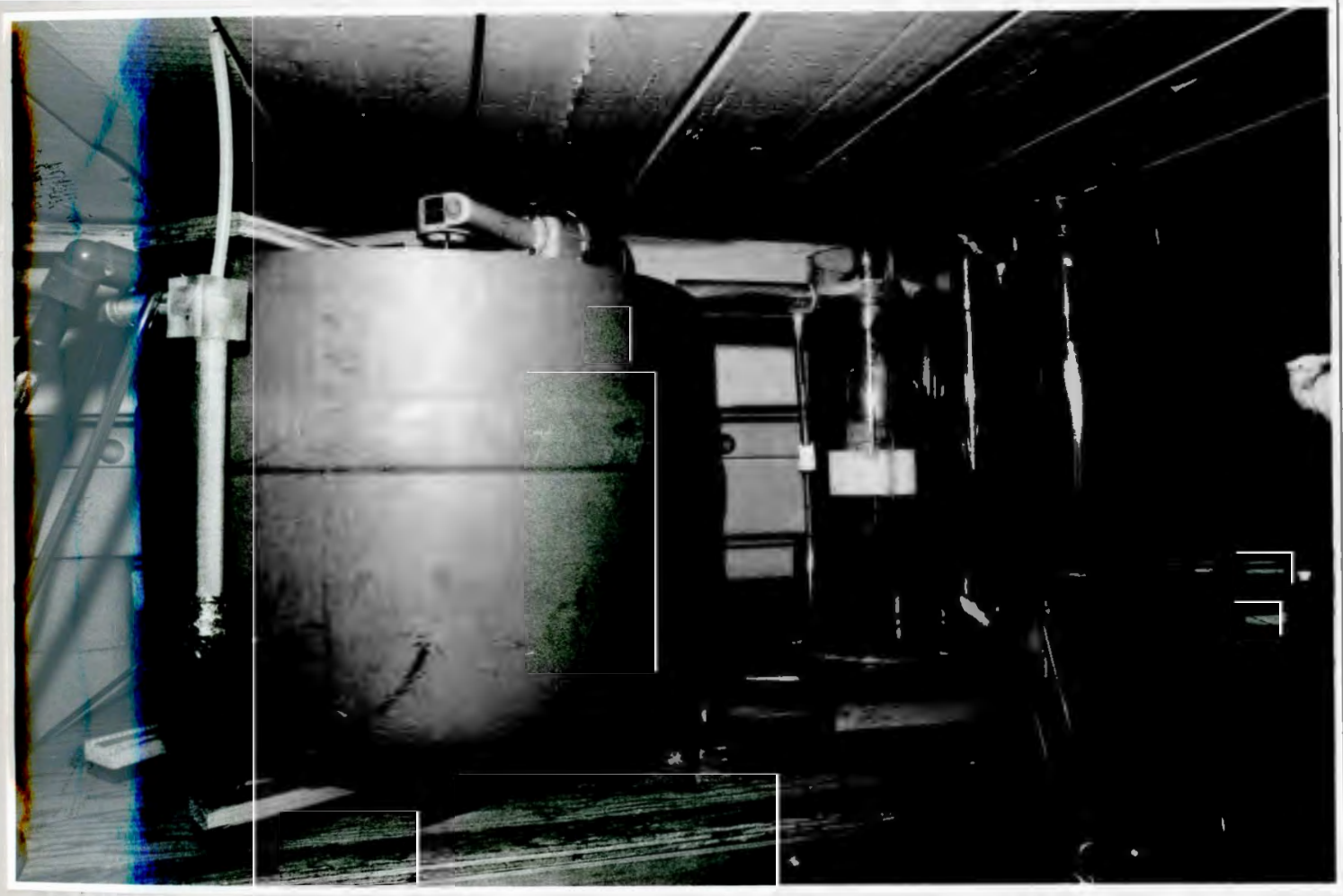

Figure 3.5 Photograph of the pilot plant pretreatment setup. 
The water from the raw water tank then entered the rapid mixing unit from the bottom side of the tank. Water was then mixed first with the alum at the pipe entrance to the tank. Mixing was provided by two $2 \times 31 / 2 \times 1 / 4$ inch plastic blades that were placed 8 inches apart on the shaft. sodium hydroxide was then added at about 8 inches from the bottom of the tank. The rapid mixing tank had the same dimensions and capacity as the raw water tank. Calibration of the rapid mixing unit was done in terms of rpm vs. G by using the torque meter. The coagulated water then moved upward and entered the slow mixing tank via a $11 / 2$ inch pipe. A sampling port was installed at the discharge pipe to allow for the sampling of the rapidly mixed coagulated water.

The coagulated water then entered the slow mixing unit from the top. The slow mixing tank was 23 inches in diameter and $171 / 2$ inches in height. The water was mixed by using a blade that was placed in the center of the tank. The blade size was $3 \times 17$ inches with a thickness of about $1 / 2$ inch. The mixing intensity was determined by calibration of the mixer using the torque meter. The coagulated/flocculated water then entered a 2 inch PVC pipe located at the bottom of the slow mixing tank that was connected to the manifold of the pilot plant filtration columns. The mixing tank was equipped with a 1 inch overflow pipe that drained the excess water and maintained a constant head. The $\mathrm{pH}$ of the overflow water was measured. 
samples of the flocculated water were collected from the tank itself using a wide mouth $25 \mathrm{ml}$ graduated pipet.

\section{sand filtration setup}

The granular bed filters consisted of three 6.0 inch I.D. glass columns. The height of each column was 54 inches. The bottom 14 inches was packed with graded gravel as a support for the uniform 17 inches of sand bed. This left 23 inches of free board to allow for bed expansion during backwash. The ends of each column were capped with a $7 / 8$ inch Teflon cap that was strapped to the glass column. Figure 3.6 is a detailed schematic diagram of a typical column and Figure 3.7 is a photograph of the three columns.

Samples for the influent analysis were collected from a sampling port located about 14 inches above the sand bed. The effluent samples were obtained from the effluent pipe of each filter downstream from the flow meter. Nine stainless steel tubes were installed in each column for head loss measurements. The tubes were $3 / 16$ inch $0 . D$. with hollow Teflon caps screwed to the end of each one. The tubes were arranged so that the difference in height between two adjacent tubes was 4 inches. The first tube was located 3 inches above the sand media to allow a measurement of the available head. The second tube was located 1 inch below the sand surface. The remaining tubes were separated by intervals of 4 inches for the full depth of the sand and gravel descended on a spiral curve surrounding the inside 


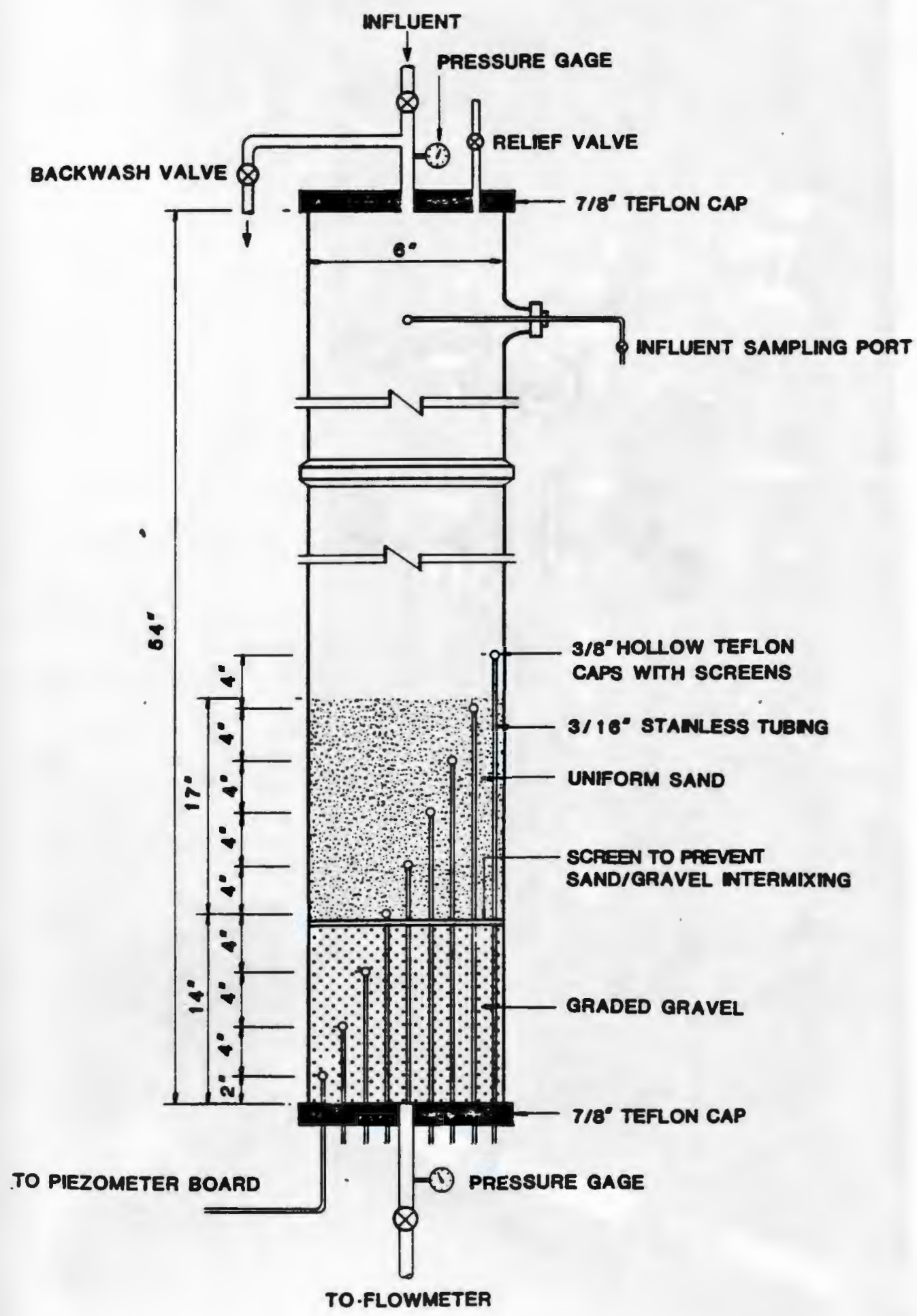

Figure 3.6 Detailed schematic of a typical pilot plant filtration column. 


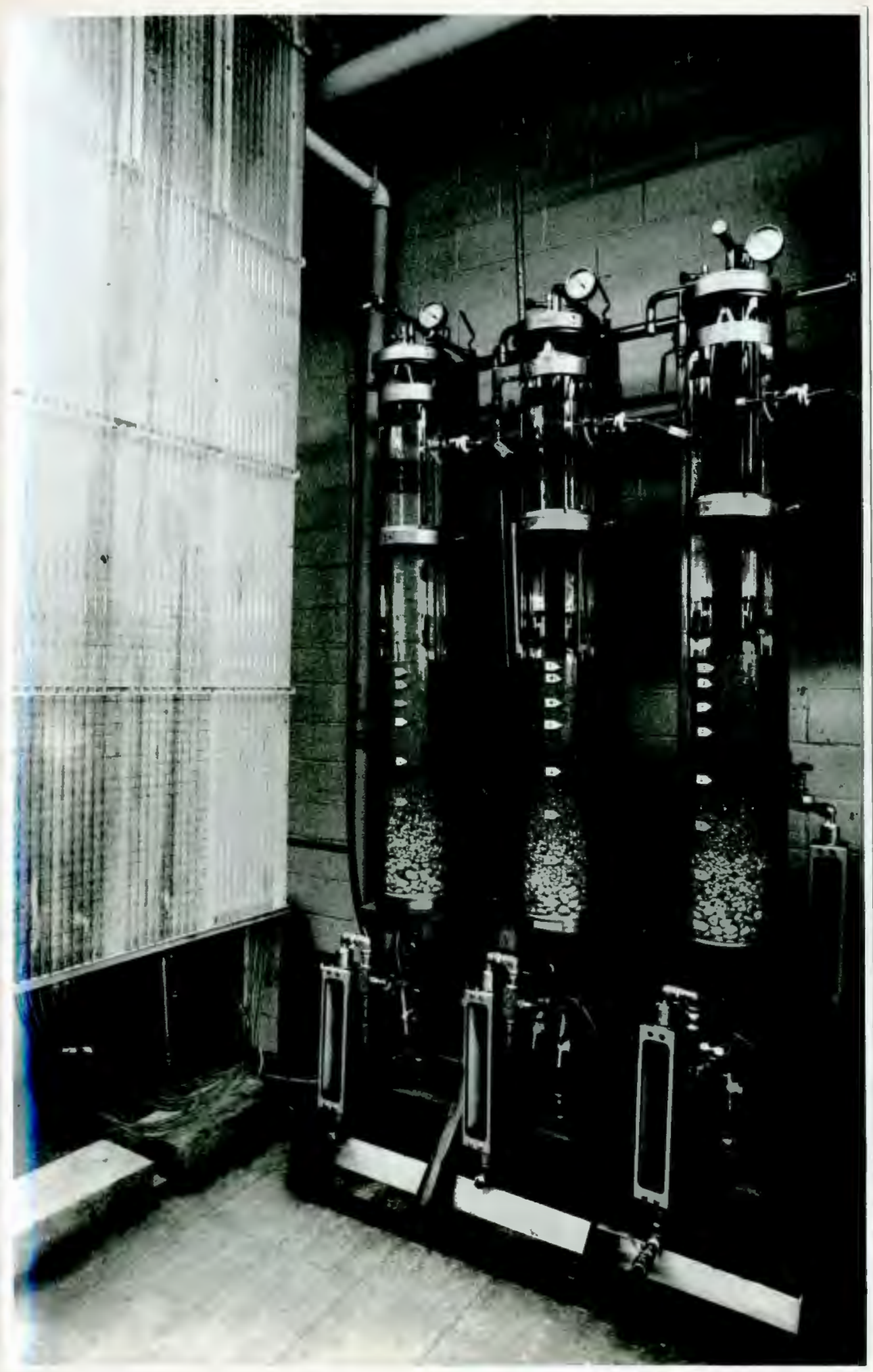

Figure 3.7 Photograph of the pilot plant sand filtration setup. 
wall of the column about $11 / 2$ inches away.

The hollow Teflon caps were $3 / 8$ inch $0 . D$. and $1 / 4$ inch I.D. Both ends of the hollow Teflon caps were covered with a stainless steel screen which had openings slightly smaller than the lowest media size used in the filters to prevent sand clogging. A stainless steel screen was installed between the sand and gravel media to prevent the intermixing of the two during backwash. The screen utilized had mesh equivalent to a U.S. standard sieve No. 14.

The tubes were connected below each column to $1 / 4$ inch flexible tubing that were connected to the piezometer board. Each tubing was connected to glass tubings that extended the whole length of the piezometer board. The piezometer board measured 12 feet high by 3 feet in width.

Water flow through each column was controlled by a flow meter that was installed below each column. The flow meters were a product of Emerson Electric Co., Hatfield, PA. The range of flow that could be measured was $0-2.00 \mathrm{gpm}$. The backwash flow meter had a range of 0-5.00 gpm due to the need for higher flow rates during backwashing.

Each column had a 14 inch layer of graded gravel to support the sand bed. The gradation of the gravel layer was as follows: 
Layer No.

Depth (in)

1 ( Top)

2

3

4

5 (Bottom)

2
2
3
3
4

Gravel Size

$\begin{array}{ll}\text { P10 } & \text { R14 } \\ \text { P1/16 } & \mathrm{R} 1 / 8 \\ \mathrm{P} 1 / 8 & \mathrm{R} 1 / 4 \\ \mathrm{P} 1 / 4 & \mathrm{R} 1 / 2 \\ \mathrm{P} 1 / 2 & \mathrm{R} 1\end{array}$

The sand media used in the three columns had the following, sieve analysis:

$\begin{array}{lll} & \text { U.S. Standard } & \text { Avg. grain size } \\ \text { Filter No. } & \text { Sieve No. }(P-R) & (\mathrm{mm})\end{array}$

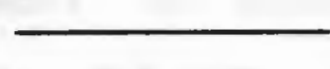

1

2

3

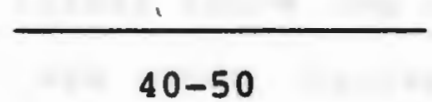

$40-50$

$30-40$

$16-20$
0.35

0.50

1.00

The porosity of the sand was calculated from the volume of the solids per unit volume of sand bed. The volume of solids is the weight of sand present in the column divided by the specific gravity of the sand. The specific gravity of the sand was taken as $2.65 \mathrm{gm} / \mathrm{cu}$. $\mathrm{cm}$. For fine media (size $0.35 \mathrm{~mm}$ ), the porosity was calculated to be 0.48 and for the medium (size $0.50 \mathrm{~mm}$ ) and coarse media (size 1.00 $\mathrm{mm})$, the porosity was 0.47. Both sand and gravel were supplied by Jesse Morie and Son, Inc. of Mauricetown, NJ which supplies filtering materials for water treatment 
plants including the plant at scituate.

\section{Sand preparation}

The sand was received in $100 \mathrm{lb}$ sacks that contained a mixture of different grain sizes. Therefore, it was necessary to sieve the sand to obtain the desired grain sizes. This was done by hand sieving 100 grams at a time using two sieves. One to pass all the grains and the other to retain the size needed. The sand then was washed with tap water to get rid of all the dust and foreign objects, then was allowed to dry in the oven at $70^{\circ} \mathrm{F}$. The dry sand then was placed in the appropriate column so that the depth of media was about 5 inches above the required depth of 17 inches. The sand bed was then backwashed for 1 hour. Backwashing resulted in the expansion of the sand bed so that fine and less dense materials would rest on top of the heavier media. The sand bed was allowed to settle very slowly in the column, after which the top 5 inches were scraped off and discarded. The 5 inches of sand that were scraped off comprised the fine media that did not pass through the bottom sieve during sieving, as well as the media that was not sand but had a lower density with the same size as the sand. 


\subsection{Experimental procedure}

The experimental work was divided into three phases. Phase 1 was the far test experiments; phase 2 was the mixing tank experiments; and phase 3 was the pilot plant experiments. Prior to each phase there were certain parameters that needed to be investigated which had a direct influence on the outcome of the main experiments. Dilution water, dilution ratio, calibration of mixers, and the influence of rapid mixing were the major pre-experimental work that had to be performed.

\section{Jar testing}

The purpose of the jar test experiments was to study the effect of mixing intensity, coagulant dosage, $\mathrm{pH}$ and mixing time on the number of particles remaining, as well as the particle size distribution. Figure 3.8 is a flow chart diagram of the jar test experiments. In all, a series of six experiments were performed. The first three experiments were performed in order to study the influence of the mixing intensity on the remaining particles and their particle size distribution by varying the mixing time and coagulant dosages. The coagulant dosages that were used were $0,2,5$, 8, 11 , and $15 \mathrm{mg} / \mathrm{L}$ of aluminum sulfate. The solution $\mathrm{pH}$ was kept constant at 7.0 . The mixing intensities were 20,40 , and $65 \mathrm{~s}^{-1}$ for experiments 2,1 , and 3 , respectively. The fourth experiment was performed to study the influence of the solution $\mathrm{pH}$ on the same parameters using a single 


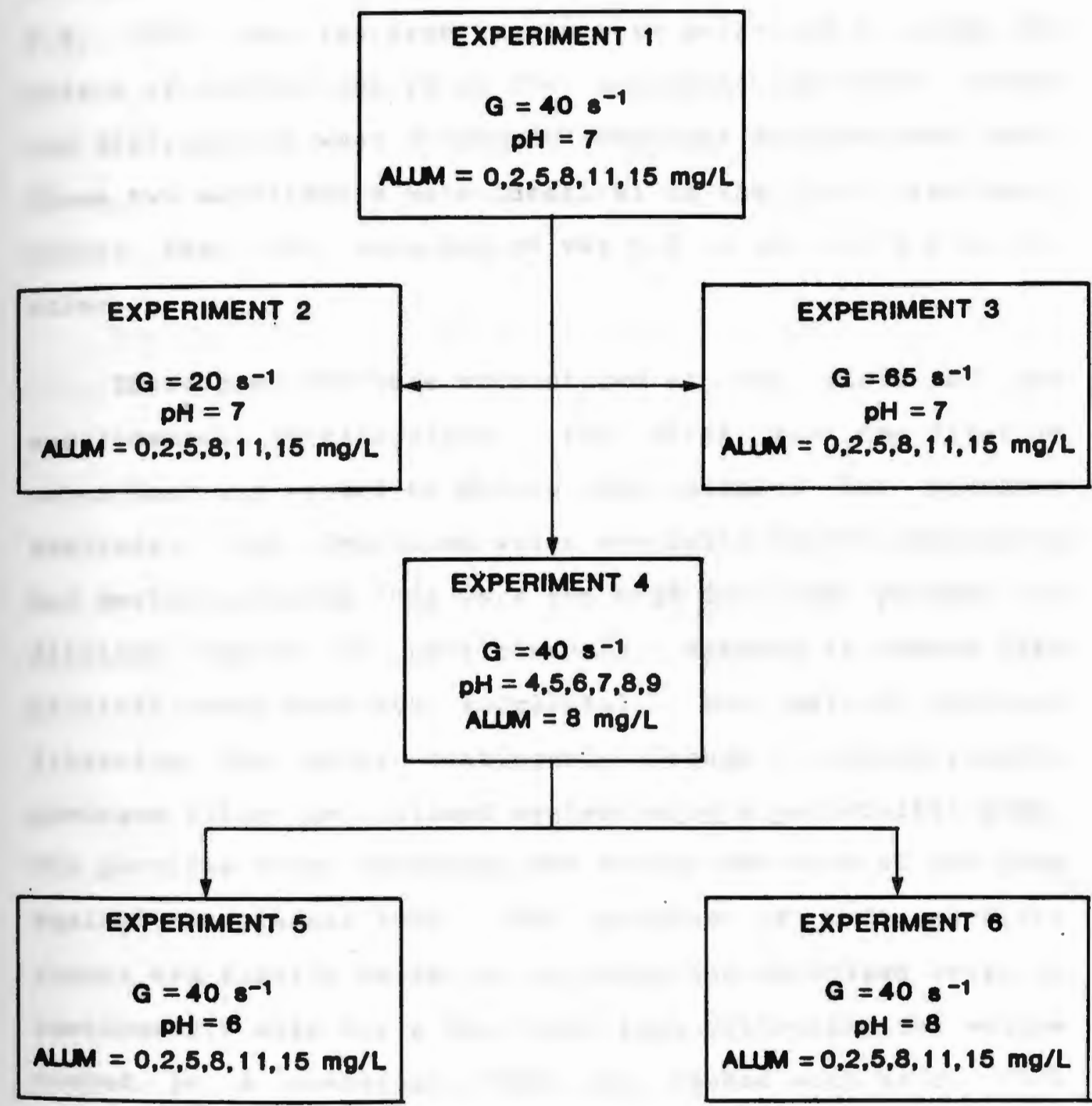

$$
\text { * } G \text { = Mixing Intensity, } 8^{-1}
$$

Figure 3.8 Flow chart of the jar test experiments. 
coagulant dosage $(8 \mathrm{mg} / \mathrm{L})$ and a mixing intensity of $40 \mathrm{~s}^{-1}$. The solution $\mathrm{pH}$ were set at $4.0,5.0,6.0,7.0,8.0$. and 9.0. The last two experiments were performed to study the effect of varying the $\mathrm{pH}$ on the remaining particle counts and distribution when different coagulant dosages were used. These two experiments were identical to the first experiment except that the solution $\mathrm{pH}$ was 6.0 in one and 8.0 in the other.

Three problems were encountered at the start of the experimental investigation. The first was the dilution water that was needed to dilute the samples for particle analysis. The deionized water available in the laboratory had particle counts that were too high for the purpose of dilution (about 20 particles/ml). Methods to reduce this particle count were not successful. One method involved filtering the water continously through a 0.45 micrometer membrane filter in a closed system using a peristaltic pump. The particle count increased due to the abrasion of the pump against the plastic tube. The problem of high particle counts was finally solved by allowing the deionized water to continuously drip for a few hours then collecting the volume needed in a container that was washed with acid. This method resulted in a particle count of less than 4 particles/ml. 
The second problem was the increase in the number of particle counts after the pH adjustment. The pH was adjusted by using calcium hydroxide (lime). It happened that the lime had particles that were causing an increase in the particle count. Switching to sodium hydroxide solved the problem. Particle counts did not increase after the addition of sodium hydroxide.

The third problem was the increase in the suspension $\mathrm{pH}$ after the start of the jar test experiments. The water used was the university tap water which was from a ground water supply. The pH of the water averaged about 5.7. The loss of carbon dioxide during stirring caused the pH to increase. The same problem was reported by other investigators such as Dempsey et al. (1984a). One solution was to add acid during the flocculation period in order to keep the pH constant over the length of the experiment. However, this method required the use of $6 \mathrm{pH}$ meters to monitor each beaker since all 6 beakers had different coagulant dosages. The solution to the problem was to mix the tap water for few hours so that most of the carbon dioxide was released to the atmosphere. By doing this, the pH increased to a range between 6.10 to 6.40 after about 3 hours of mixing. This method produced a constant pH during the entire length of the experiments. 
Dilution of the samples was necessary because the maximum total particle count which could be measured with the available sensor was 2,000 particles/ml as was recommended by the manufacturer. This is to avoid the shadowing and clustering effects. clustering is when a cluster of small particles are flowing through the sensor and counted as one large particle. Shadowing is when a larger particle shields one or more smaller particles as they pass through the sensor.

Determination of the dilution ratio was accomplished by a sequential dilution of a stock solution of the particles as was suggested by the manufacturer. With a low dilution ratio and a high particle count, the measured particle count is low due to clustering and shadowing effects. As the dilution ratio increases, the number of particles counted increases to a maximum value which then decreases with more dilution. A stock solution was prepared from the clay. Nine different concentrations were prepared from the stock solution. The dilution ratios were $1,2,4,8,16,32,64$, 128 and 256. Particle counts for each of the nine samples were performed. The same sample was analyzed three times. The three values were then totaled and were averaged for plotting purposes.

The total number of particles was plotted against the dilution ratio on a log-log paper as shown in Figure 3.9. The minimum dilution was that value corresponded to the intersection of the tangent line to the negatively sloped 


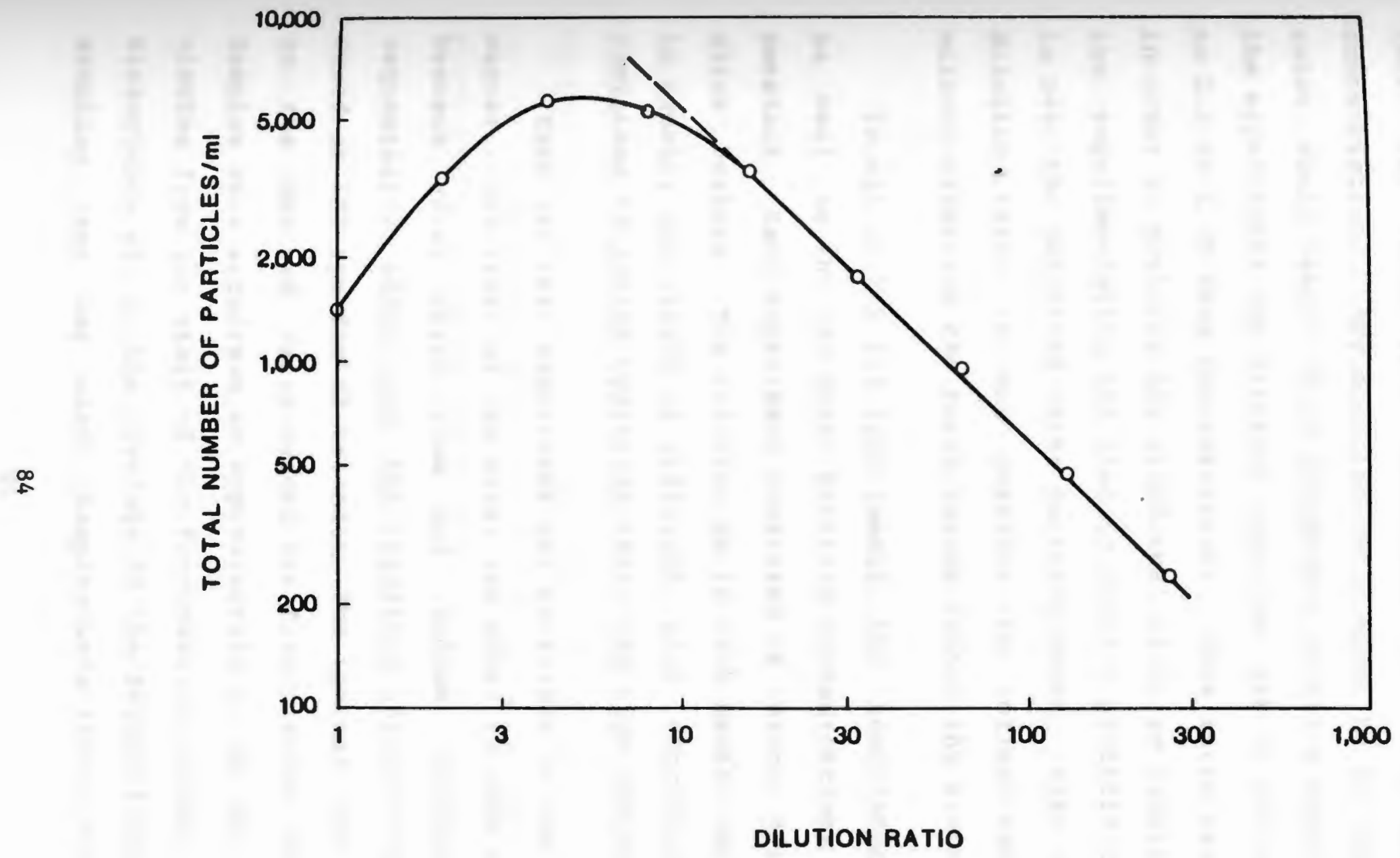

Figure 3.9 Total number of particles/ml with different dilution ratios of MIN-U-GEL 200. 
portion of the curve. In Figure 3.9 the minimum dilution ratio was about 16.0 (equivalent to $6.25 \mathrm{mg} / \mathrm{L}$ of mass concentration). Any dilution ratio equal to or above that value would result in an acceptable particle count. In all the experiments the dilution ratio was set at 40 (equivalent to $2.5 \mathrm{mg} / \mathrm{L}$ of mass concentration). This ratio was selected in order to minimize the withdrawal of large samples during the experimentation and also to avoid a particle count that is near the deionized water particle count. Also with this dilution, ratio it was possible to collect many samples without affecting the total volume within the mixing unit.

In all of the six experiments, the temperature $\left(12^{\circ} \mathrm{C}\right)$ as well as the raw water particle concentrations were kept constant. Each experiment consisted of using 6-one liter glass beakers. The solution $\mathrm{pH}$ in each beaker was adjusted to counter the effect of different alum concentrations by additions of sodium hydroxide after the alum addition.

Each jar test experiment was performed in the following manner. One liter of raw water was added to each of the six beakers after which alum and sodium hydroxide were sequentially added and the resulting solution was rapidly mixed at $100 \mathrm{rpm}$ for 45 seconds. The rpm was then adjusted to the desired value needed for flocculation (30-70 rpm). Samples were withdrawn at approximately 1, 10, 20, 30 and 40 minutes from the start of the flocculation period. To avoid disturbance of, or the breakage of the formed flocs, extreme sampling care was used. Samples were slowly withdrawn by 
using wide mouth $25 \mathrm{ml}$ graduated pipets. All samples were withdrawn at a depth of one inch below the surface. Each water sample was exactly $20 \mathrm{ml}$ in volume. The $20 \mathrm{ml}$ samples were diluted with deionized water containing a known particle count using $190 \mathrm{ml}$ special cylindrical sampling bottles. The sampling glass bottles had a plastic cap with a teflon lining so that particles would not be released during the capping and uncapping of the bottle. The bottles were first cleaned with laboratory detergent, then rinsed with distilled water and acid solution, after which they were baked in an oven for at least 2 hours.

At the end of each series of experiments, water samples were analyzed for $\mathrm{pH}$ to make sure that the $\mathrm{pH}$ did not change during the time of experimentation. In cases where the $\mathrm{pH}$ was different from the required value, the experiment was repeated. Throughout the experiment there was no noticeable change in the solution temperature. After the measurement of the $\mathrm{pH}$, a particle size analysis was quickly performed so as to minimize any settling or flocculation that could take place within the sampling bottles.

Each sample was sequentially analysed three times utilizing the HIAC PC-320 particle size analyzer equipped with an automatic bottle sampler. Each time, 30 ml was allowed to pass through the particle counter sensor and readings of the number of particles were stored in 12 different size intervals ranging from 2-150 microns. Since the samples were diluted, the actual number of particles 
present in the sample were computed by subtracting the dilution water particles from the sample. The following formula was used in the computation:

$$
\mathbf{N}_{A}=\frac{\mathbf{v}_{T} \mathbf{N}_{\mathbf{P}}-\mathbf{v}_{\mathbf{D}} \mathbf{N}_{\mathbf{D}}}{\mathbf{v}_{S}}
$$

where

$N_{A}$ - actual number of particles present in the original sample before dilution in a specific size range

$N_{p}=$ number of particles present in the diluted sample in a specific size range

$N_{D}=$ number of particles present in the deionized water in a specific size range

$V_{T}=$ total volume in the sampling bottle

$v_{D}=$ volume of deionized water

$v_{s}=$ volume of original sample

The actual number of particles in each size interval as well as the slope of the distribution function ( $\beta$ ) were calculated for each reading by using a computer program. The computer program is listed in Appendix A. The average of the three readings were then used in plotting the total number of particles and the distribution slope against mixing time.

The particle counter sensor was cleaned before each experiment by carefully blowing air through the opening. The flow of sample through the sensor was maintained 
constant by regular calibration. For the sensor used, the flow had to be $150 \pm 50 \mathrm{ml} / \mathrm{min}$.

The first experiment was used to quantify the standard conditions. The mixing intensity, $40 \mathrm{~s}-1$, is a typical value used in practice. The $\mathrm{pH}$ of 7.0 is within the range for optimum turbidity removal by aluminum sulphate.

\section{Mixing tank experiments}

The purpose for this phase of experimental investigation was to study the influence of mixing intensity and time on the particle size distribution when utilizing a pilot-scale reactor vessel. The reasons for using a large mixing tank as follows:

(1) It was possible to obtain as many samples as possible in a short time interval;

(2) the mixing time can be extended without affecting the remaining solution; and

(3) the same tank was used in the pilot plant studies.

Four different experiments were performed to study the effect of mixing intensity on the particle size distribution when using a larger size tank. The alum dose $(8.00 \mathrm{mg} / \mathrm{L})$, the solution $\mathrm{pH}$, and the particle concentration (about 20,000 particles/ml) were all kept constant in each of the four experiments. The mixing intensities used were 25,50 , 75 , and $100 \mathrm{~s}^{-1}$. The choice of a $17 \times 3$ inch mixing blade size was based on the fact that it gave homogenous mixing in 
the tank and the fact that it resulted in the required mixing intensities with the available rpm which could be supplied by the mixer.

The velocity gradient in the mixing tank was determined by using the torque meter at different rotational speeds. The torque meter was placed on the shaft between the motor and the blade. The velocity gradient was then calculated using equation 5 in the literature review chapter. The $G$ values are shown in Table 3.2 for the $17 \times 3$ inch blade size. Figure 3.10 is a plot of the velocity gradient as a function of the shaft rpm for two different blade sizes for the same tank size and water temperature. Since the same calibration curve was used when conducting pilot plant experiments, an investigation of the influence of continuous flow on the relationship between the shaft speed and velocity gradient was performed. No differences from the batch results were observed at the maximum flow expected through the system.

The same procedure of sampling and particle analysis was followed, as was used in the jar test experiments. The only differences were the sampling time and the location of sampling within the tank. Samples were collected from a fixed location of 4 inches away from the shaft and 5 inches below the surface. 
Table 3.2- Calibration of the slow Mixing Unit

\begin{tabular}{lccc}
\hline RPM & $\begin{array}{c}\text { Torque } \\
(\text { oz-in) }\end{array}$ & $\begin{array}{c}\text { Power Input } \\
(\mathrm{ft}-1 \mathrm{~b} / \mathrm{s})\end{array}$ & Mixing $\begin{array}{c}\text { Intensity, G } \\
(\mathrm{s}-1)\end{array}$ \\
\hline 11.70 & 5.00 & 0.032 & 19.0 \\
14.30 & 7.50 & 0.058 & 25.7 \\
17.10 & 10.00 & 0.093 & 32.5 \\
22.20 & 15.00 & 0.180 & 45.3 \\
25.00 & 17.50 & 0.240 & 52.0 \\
27.30 & 20.00 & 0.300 & 58.1 \\
31.60 & 25.00 & 0.430 & 69.8 \\
35.30 & 28.00 & 0.540 & 78.2 \\
37.50 & 32.50 & 0.660 & 86.8 \\
40.00 & 39.00 & 0.850 & 98.1 \\
42.90 & 47.50 & 1.110 & 112.2 \\
48.00 & 52.50 & 1.370 & 124.8 \\
52.20 & 62.50 & 1.780 & 142.0 \\
57.10 & 75.00 & 2.330 & 162.7 \\
\hline & & & \\
\hline
\end{tabular}

Note: water temperature: $12^{\circ} \mathrm{C}$

Tank volume: 25.8 gallons ( 97.5 liters)

Blade size: $17 \times 3$ in. 


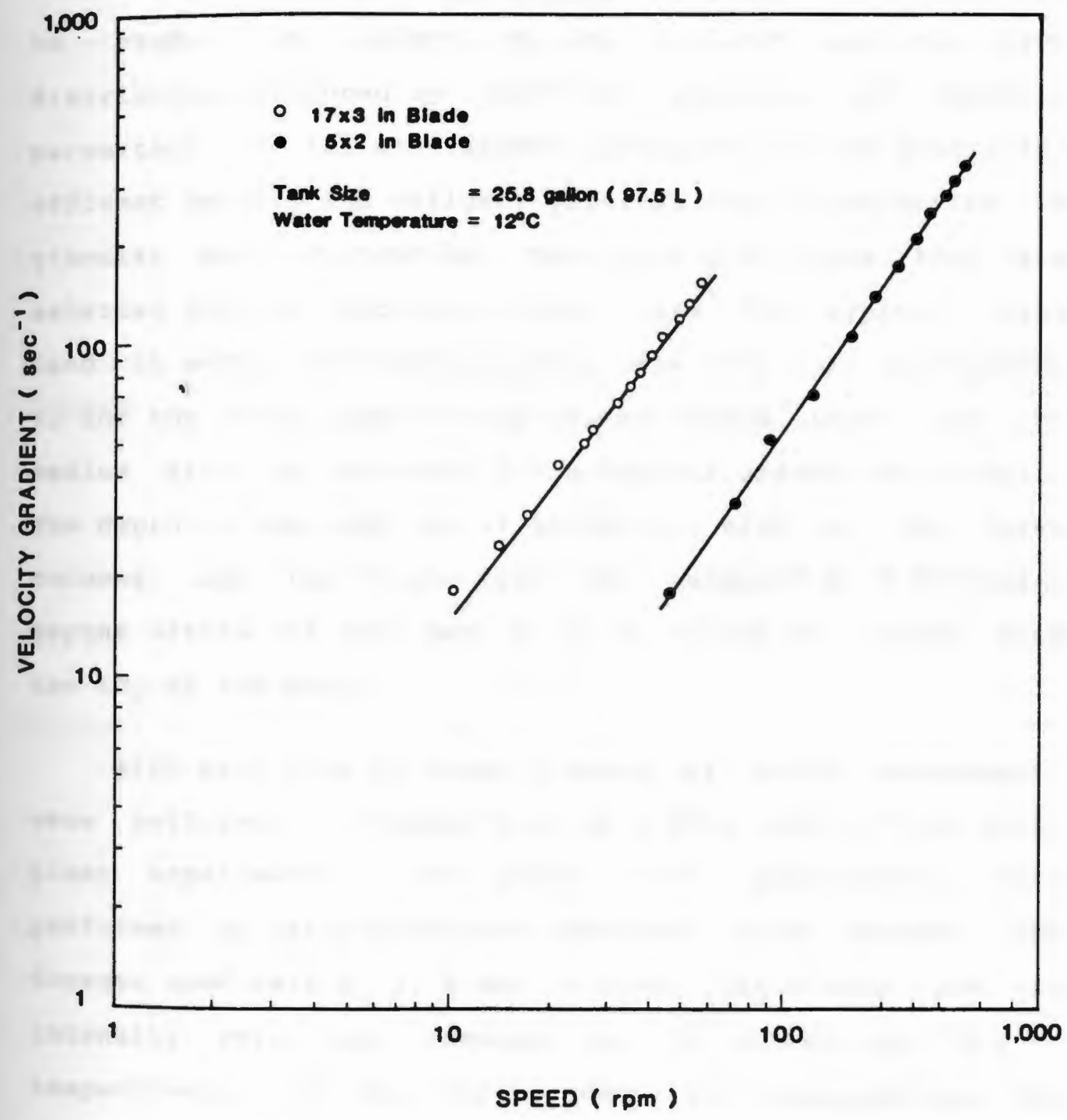

Figure 3.10 Velocity gradient versus rpm for the pilot plant mixing tank with two different blade sizes. 


\section{Pilot-plant operation}

The purpose of this phase of the experimental work was to study the effect of the influent particle size distribution produced by both the physical and chemical parameters in the pretreatment processes on the head loss, effluent quality and effluent particle size distribution in granular media filtration. The three grain sizes that were selected for the filtration units were the typical sizes used in actual treatment plants. The fine size corresponds to the top layer, coarse size to the bottom layer and the medium size in between for a typical graded sand filter. The depth of the sand was 17 inches in each of the three columns, and the head loss was measured at 5 different depths within the sand bed; $1,5,9,13$ and 17 inches from the top of the media.

with each size of sand, a total of eight experiments were performed. Figure 3.11 is a flow chart of the pilot plant experiments. The first four experiments were performed by using different coagulant (alum) dosages. The dosages used were $0,2,8$ and $16 \mathrm{mg} / \mathrm{L}$. The mixing time and intensity were kept constant at 30 minutes and $50 \mathrm{~s}^{-1}$, respectively. In the fifth and sixth experiments, the mixing intensity was set at 25 and $100 \mathrm{~s}^{-1}$. The coagulant dosage and mixing time were kept constant at $8 \mathrm{mg} / \mathrm{L}$ and 30 minutes. In the last two experiments, the mixing time was set at 15 and 45 minutes. The coagulant dosage and the mixing intensity were kept constant at $8 \mathrm{mg} / \mathrm{L}$ and $50 \mathrm{~s}^{-1}$. 


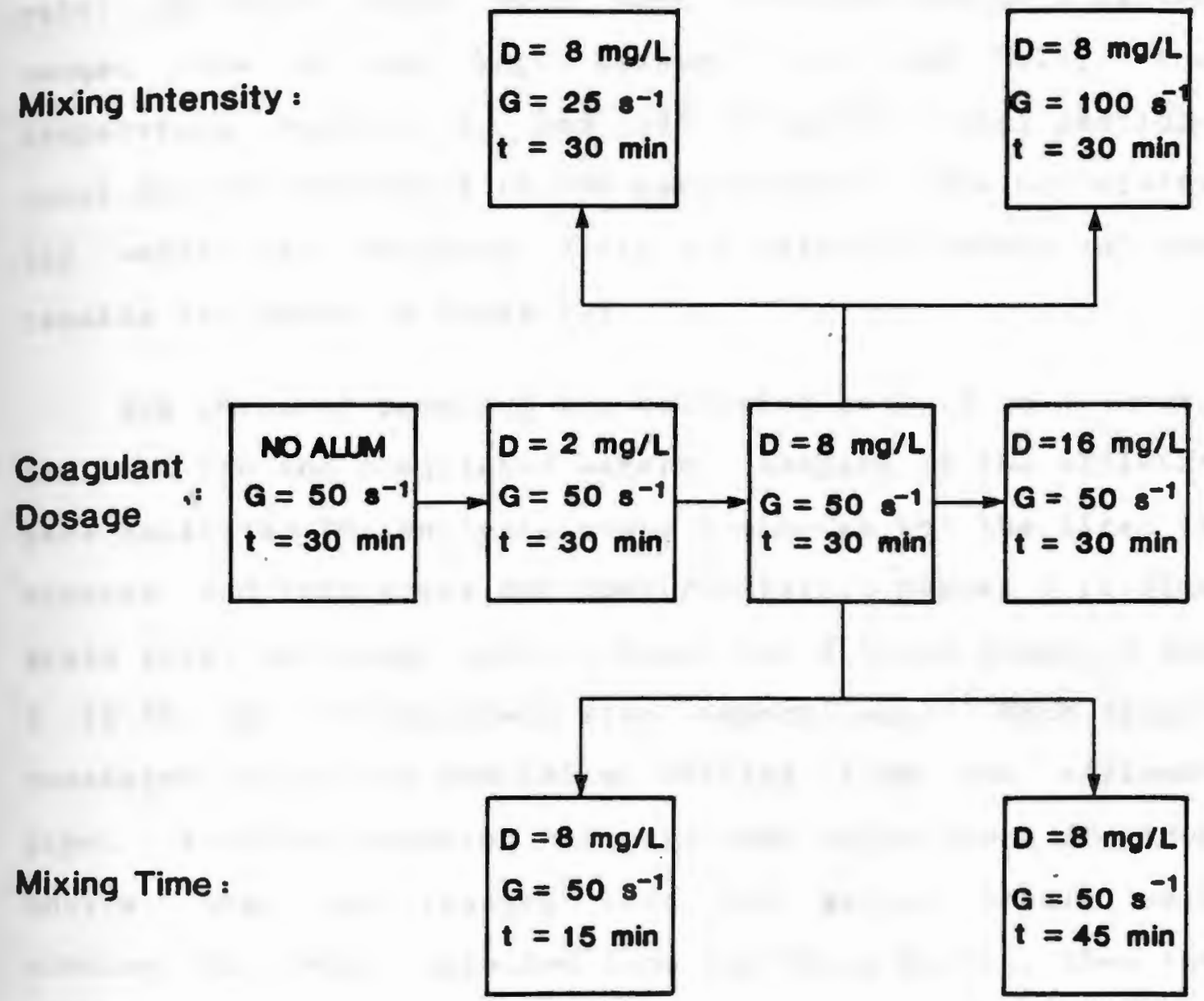

\section{KEY :}

G = Mbxing intenaity, $8-1$

$t=$ Mixing Tine, minutes

D = Coagulant Dosage, $\mathrm{mg} / \mathrm{L}$

Figure 3.11 Flow chart of the sand filtration runs. 
In all of the 24 runs, the solution $\mathrm{pH}$, temperature and raw water particle count were kept constant within a narrow range. The $\mathrm{pH}$ was kept between 6.90 and 7.15 , the temperature between 12 and $14^{\circ} \mathrm{C}$ and the total particle count had an average of 16,700 particles/m1. The university tap water was analyzed daily for particle counts and the results are shown in Table 3.3 .

The influent sampling was collected every 2 to 4 hours for both raw and coagulated waters. Samples of the effluent were coliected for analysis every 5 minutes for the first 15 minutes and then every one hour for filter number 1 ( $0.35 \mathrm{~mm}$ grain size) and about every 2 hours for filters number 2 and $3(0.50$ and $1.00 \mathrm{~mm}$ grain size respectively). Each sample consisted of filling two-190 ml bottles from the effluent pipe. Particle counting was performed three times for each bottle. When the results from the second bottle were similar to those obtained from the first bottle, then the last three readings were discarded. Therefore, each point on the curves related to the effluent quality, to be presented in the next chapter, represents the average of 3 to 6 values.

The flow rate through the media was kept constant at $0.50 \mathrm{gpm}$. This was equivalent to $2.55 \mathrm{gpm} / \mathrm{sq} \mathrm{ft}$ which is typical of what is used in most treatment plants. With the size of the pilot plant mixing tank, it was possible to vary the detention time from 15 to 45 minutes. The flow rate was kept constant by periodically adjusting the flow to 0.50 
rable 3.3- University Tap Water Particle size Analysis*

Channel Average size Number of particles/ml standard Numbe 5

(Microns) Maximum Minimum Average

Deviation

\begin{tabular}{rrrrrr}
\hline 1 & 3.5 & 216.10 & 2.40 & 27.40 & 40.05 \\
2 & 7.5 & 18.01 & 0.63 & 3.53 & 4.15 \\
3 & 12.5 & 3.48 & 0.01 & 0.50 & 0.74 \\
4 & 17.5 & 1.54 & 0.00 & 0.20 & 0.29 \\
5 & 22.5 & 0.57 & 0.00 & 0.09 & 0.12 \\
6 & 27.5 & 0.20 & 0.00 & 0.03 & 0.05 \\
7 & 32.5 & 0.10 & 0.00 & 0.02 & 0.03 \\
8 & 37.5 & 0.04 & 0.00 & 0.01 & 0.02 \\
9 & 45.0 & 0.11 & 0.00 & 0.01 & 0.03 \\
10 & 62.5 & 0.04 & 0.00 & 0.01 & 0.02 \\
11 & 87.5 & 0.00 & 0.00 & 0.00 & - \\
12 & 125.0 & 0.00 & 0.00 & 0.00 & - \\
\hline
\end{tabular}

* Based on 90 samples 
gpm.

For any particular run, the total flow rate was determined based on the detention time required plus a fraction for the overflow. As an example, for the run where the required detention time was 30 minutes, the total flow rate was set as follows: for the 25.8 gallon mixing tank and 30 minutes of detention time, the flow rate required is $0.86 \mathrm{gpm}(25.8 / 30)$. The flow through the filter was 0.50 gpm, so $0.36 \mathrm{gpm}(0.86-0.50)$ was wasted through the overflow pipe. The overflow pipe was installed at the bottom of the tank so that all water entering the mixing tank was mixed for a time equal to the detention time (see Figure 3.3). The chemical requirements were set according to the total flow rate, and the water temperature was set by adjusting the cold and hot water valves.

Since the suspension was rapidly mixed, it was necessary to investigate the influence of rapid mixing on the particle size distribution of the coagulated water since with different detention times, the flow rate was different. This resulted in different detention times in the rapid mixing unit which could influence the rate of initial flocculation. To accomplish this, two flow rates were selected that would give different detention times. These were 0.58 and $1.72 \mathrm{gpm}$ resulting in 2.74 and 0.91 minutes of detention time in the rapid mixing unit. samples were collected for three different mixing intensities; 200 ; 500 and $700 \mathrm{~s}-1$. The calibration of the rapid mixing unit was 
performed in advance and the calculation of the velocity gradient for different speeds is listed in Table 3.4 and plotted in Figure 3.12. For each mixing intensity, three samples were collected and each was analyzed three times giving a total of nine observation points for each sample. The results indicated that after a Gt value of 28,000 , there was no significant change in the particle size distribution. Gt being defined as the mixing intensity multiplied by the mixing time. Therefore, during the operation of the pilot plant, Gt, was kept constant at 28,000 by adjusting the speed of the rapid mixer. The temperature $\left(12^{\circ} \mathrm{C}\right)$ and $\mathrm{pH}(7.0)$ were kept constant during the length of experiments.

The three peristaltic pumps that pumped clay suspensions, alum and sodium hydroxide were calibrated prior to the start of the pilot plant experiments. This was done volumetrically. The pump controller was set for the desired flow rate in ml/min. To assure precise measurements, the pump was tested by recording the time required to deliver exactly 4 liters of solution. The tubes were changed regularly to avoid changes in flow rates and possible leaks.

Prior to the start of filtration, the system was allowed to stabilize for at least 3 hours. This was to ensure a constant $\mathrm{pH}$, temperature, water particle count and desired mixing intensity. 
Table 3.4-Calibration of the Rapid Mixing Unit

\begin{tabular}{lccc}
\hline RPM & $\begin{array}{c}\text { Torque } \\
(\text { oz-in) }\end{array}$ & $\begin{array}{c}\text { Power Input } \\
(\mathrm{ft}-1 \mathrm{~b} / \mathrm{s})\end{array}$ & $\begin{array}{c}\text { Ming Intensity, G } \\
\left(\mathrm{s}^{-1}\right)\end{array}$ \\
\hline 147 & 5 & 0.40 & 249 \\
167 & 7 & 0.64 & 314 \\
194 & 9 & 0.95 & 384 \\
221 & 10 & 1.20 & 432 \\
239 & 12 & 1.56 & 493 \\
258 & 15 & 2.11 & 572 \\
276 & 17 & 2.56 & 630 \\
295 & 20 & 3.22 & 706 \\
309 & 22 & 3.70 & 758 \\
326 & 25 & 4.44 & 830 \\
339 & 27 & 4.99 & 957 \\
361 & 30 & 5.90 & 880 \\
\hline
\end{tabular}

Note: Water temperature: $12^{\circ} \mathrm{C}$

Tank volume: 1.59 gallon (6 liters)

Blade size: $2-31 / 2 \times 2$ in. 


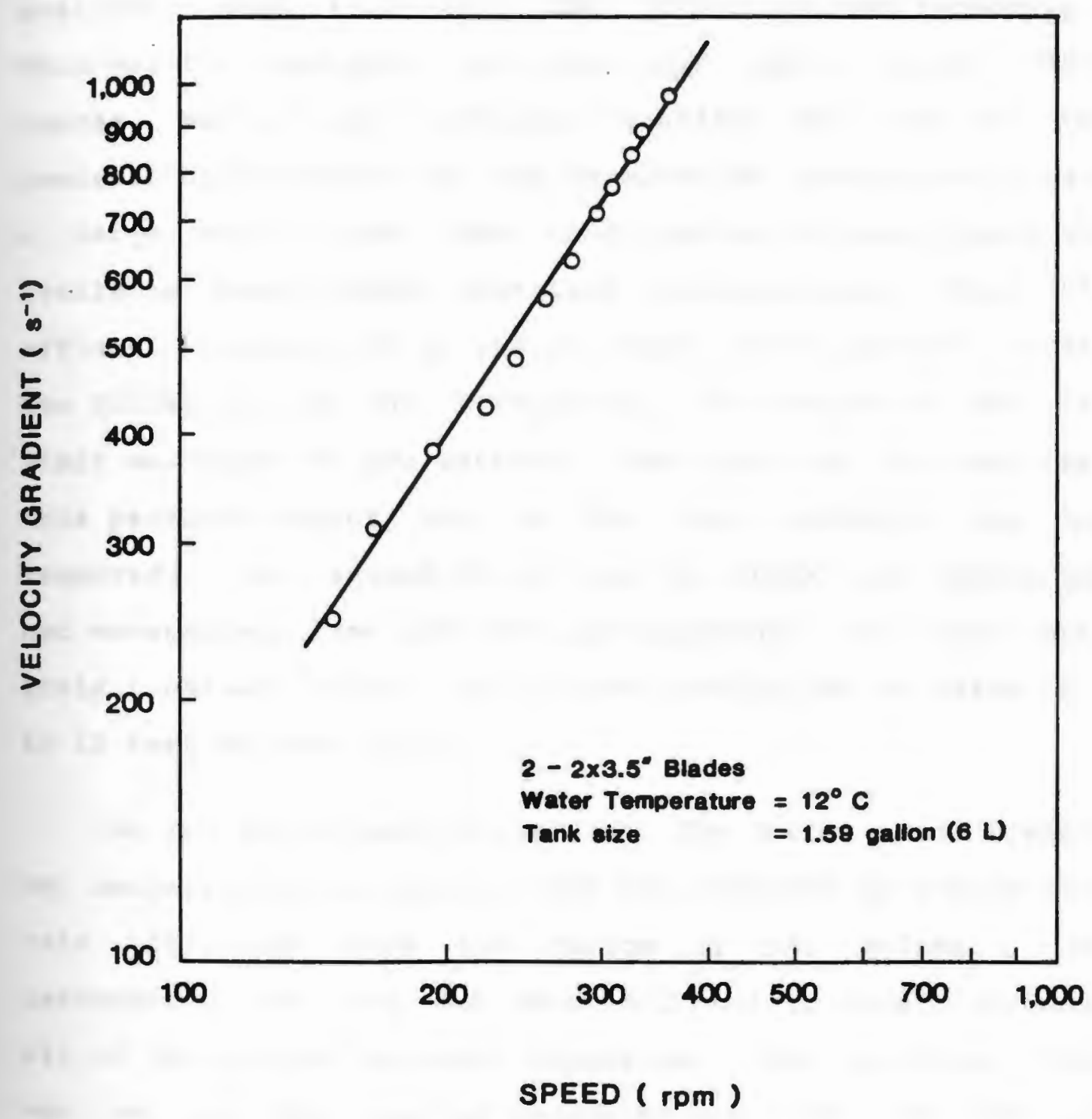

Figure 3.12 velocity gradient versus rpm for the rapid mixing unit. 
The filter run termination was based on two performance parameters, the effluent quality and the head loss. When the head loss across the entire sand bed reached the available head $(7.5 \mathrm{ft})$, the filter run was terminated. This was the case with the fine and medium sand. With coarse media the effluent quality was used as the controlling parameter for the termination, because with such a large media size, the head loss would never reach the avallable head before particle breakthrough. When the effluent, reached $10 \%$ of the raw water total particle count, the filter run was then terminated. The choice of the $10 \%$ limit was based on two factors. The first was the fact that this particle count was so low that dilution was not required. The second factor was the filter run length was not excessively long with this arrangement. In most full scale treatment plants, the filter termination is based on 6 to 10 feet of head loss.

At the end of each filter run, the filter was cleaned by backwashing the media. This was achieved by a high flow rate introduced from the bottom of the column. The backwashing was set for about $1 / 2$ hour in order to remove all of the solids that were deposited. The backwash rate was set so that the bed expansion was 1008. For fine and medium size media, this was in the range of 3-4 gpm. For coarse media, $100 \%$ expansion was not possible so the backwash rate was set at the maximum of 5 gpm and was extended for about another 15 minutes. The bed expansion 
was about 50\%. The backwashing rates used in the study were higher than what is used in practice. The reason for higher backwashing rates was to return the filter bed to its original state so comparisons can be made. Before the termination of backwashing, samples were taking for particle analysis to make. sure that the sand bed was free from previously deposited particles. Closure of the backwash valve was done in intervals so that the sand bed would return to its original configuration. 


\section{RESULTS AND DISCUSSION}

The results and discussion are divided into two main sections. In the first section, the results of the pretreatment (coagulation/flocculation) experiments will be discussed. In the second section, the results of the pilot plant experiments will be presented and discussed.

\subsection{Pretreatment}

The results to be presented in this section are those that were obtained from the six jar test experiments as well as those obtained from the four pilot plant mixing tank experiments. The discussion will be focused on the change of the total number of particles as well as the change in the distribution slope, $\beta$, with different operational parameters.

\subsubsection{Jar Test Results}

The purpose of the jar test experiments was to study the effect of mixing intensity, coagulant dosage, pH, and mixing time on both the number of particles remaining in solution as well as the particle size distribution. 
The first experiment was used to define and quantify the standard conditions. The data that were collected from all the six jar test experiments are listed in Appendix B. The raw data were fitted to first and second order polynomial functions. The best fit was found to be a second order polynomial function. The rationale for this kind of fitting as well as a discussion of the statistics that compare the two fittings will be presented in the following section. Each point on the curves represents the average of three values. An attempt to show the maximum and minimum around each point resulted in confusion from the crowding of lines. The curve that represents no coagulant addition was not fitted to any equation, instead, the mean value of all the observation data was used in plotting the line.

For each experiment, there will be three figures. The first figure is a plot of the remaining total number of particles against mixing time. The second figure is a plot of the distribution slope (from equation 15 in the literature review chapter) against mixing time. The last figure is a plot of the remaining total number of particles and the distribution slope at two different mixing times against the coagulant dosage or suspension pH. The two mixing times that were used to plot the third figure were 30 minutes and the mixing time that occurs at the zero slope of the curves in the first two figures. In most of the six experiments, 30 minutes was about the optimum mixing time. 
Figure 4.1 is a plot of the total number of particles per ml against mixing time for different alum dosages. When only sodium hydroxide was added and no alum was used, there was no significant change in the total number of particles with mixing and time. This indicates that no flocculation took place even with the addition of the $5.0 \mathrm{mg} / \mathrm{L}$ of sodium hydroxide needed to raise the solution pH of the raw water from 6.15 to 7.00

Figure 4.2 is a plot of the distribution slope, $\beta$, against mixing time for the same run. When only sodium hydroxide was added, the distribution slope was shifted slightly from the raw water toward a value that would indicate either an increase in the number of small particles or a decrease in the number of the large ones. Looking at Table B-1 in Appendix $B$, it can be seen that there is a decrease in the number of the large size particles with time. The reason for this decrease is the fact that some particles settle to the bottom during the mixing of the suspension. This causes a decrease in the large size particles, hence an increase in the distribution slope.

When $2.0 \mathrm{mg} / \mathrm{L}$ of alum were added, there was a strong mixing time influence on flocculation. This was indicated by the change in both the remaining total number of particles as well as the decrease in the distribution slope. However, the rate of flocculation was not high enough to reach a minimum particle count at a short mixing time. This is attributed to the fact that the ratio of the coagulant 


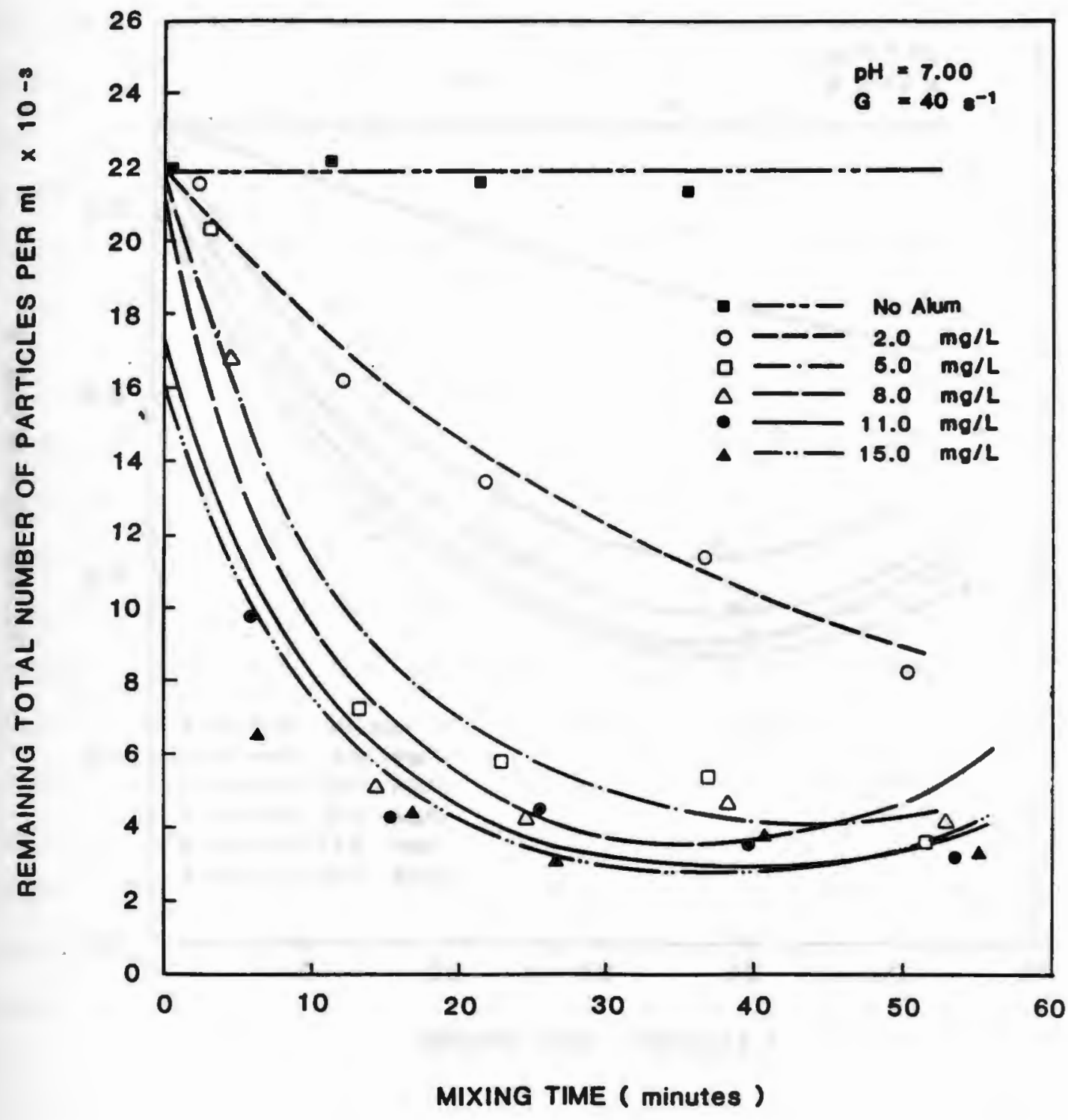

Figure 4.1 Remaining total number of particles/ml versus mixing time for far test experiment number 1 . 


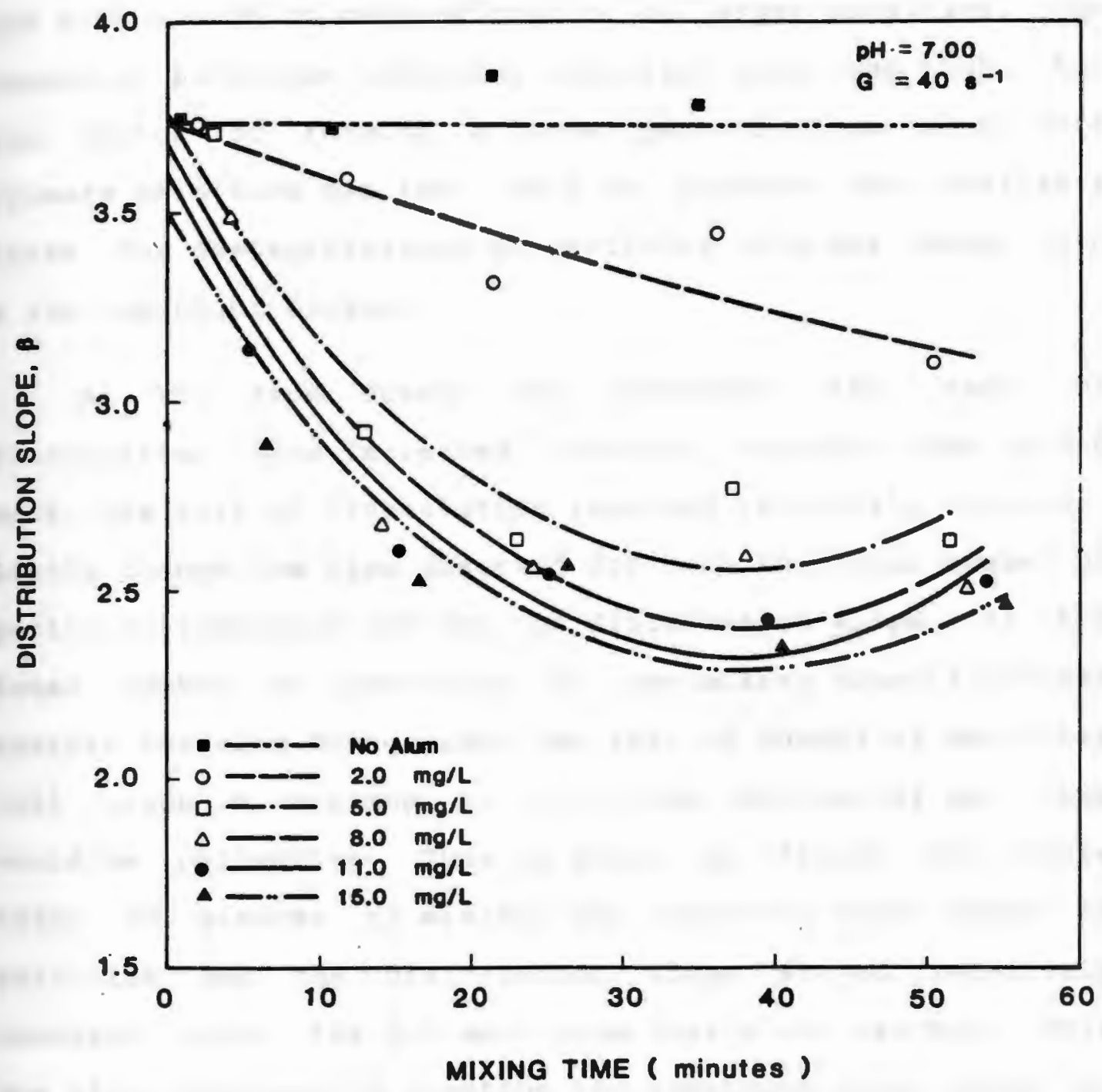

Figure 4.2 Distribution slope versus mixing time for jar test experiment number 1 . 
dose to the concentration of the particles in the water was low, therefore, the chance of contact between particles was not high enough to cause formation of larger particles. The chance of particles colliding with each other was high, but the chance of forming a larger particle from two or more primary particles was low. This is because the available sites for destabilization of particles were not enough with a low coagulant dosage.

As the alum dosage was increased, the rate of flocculation also increased. However, beyond a dose of 8.0 $\mathrm{mg} / \mathrm{L}$, the rate of flocculation remained relatively constant. Little change was also observed for both the total number of particles remaining and for the distribution slope. If the total number of particles at any mixing time is plotted against the alum dosage then the rate of change of particles will reach a minimum in which the addition of more alum would be ineffective. This is shown in Figure 4.3 where after 30 minutes of mixing, the remaining total number of particles and the distribution slope stayed relatively constant after the $8.0 \mathrm{mg} / \mathrm{L}$ alum dosage was reached. This was also confirmed by plotting the remaining total number of particles and the distribution slope against the coagulant dosage at the zero slope of the curves shown in Figures 4.1 and 4.2 . 


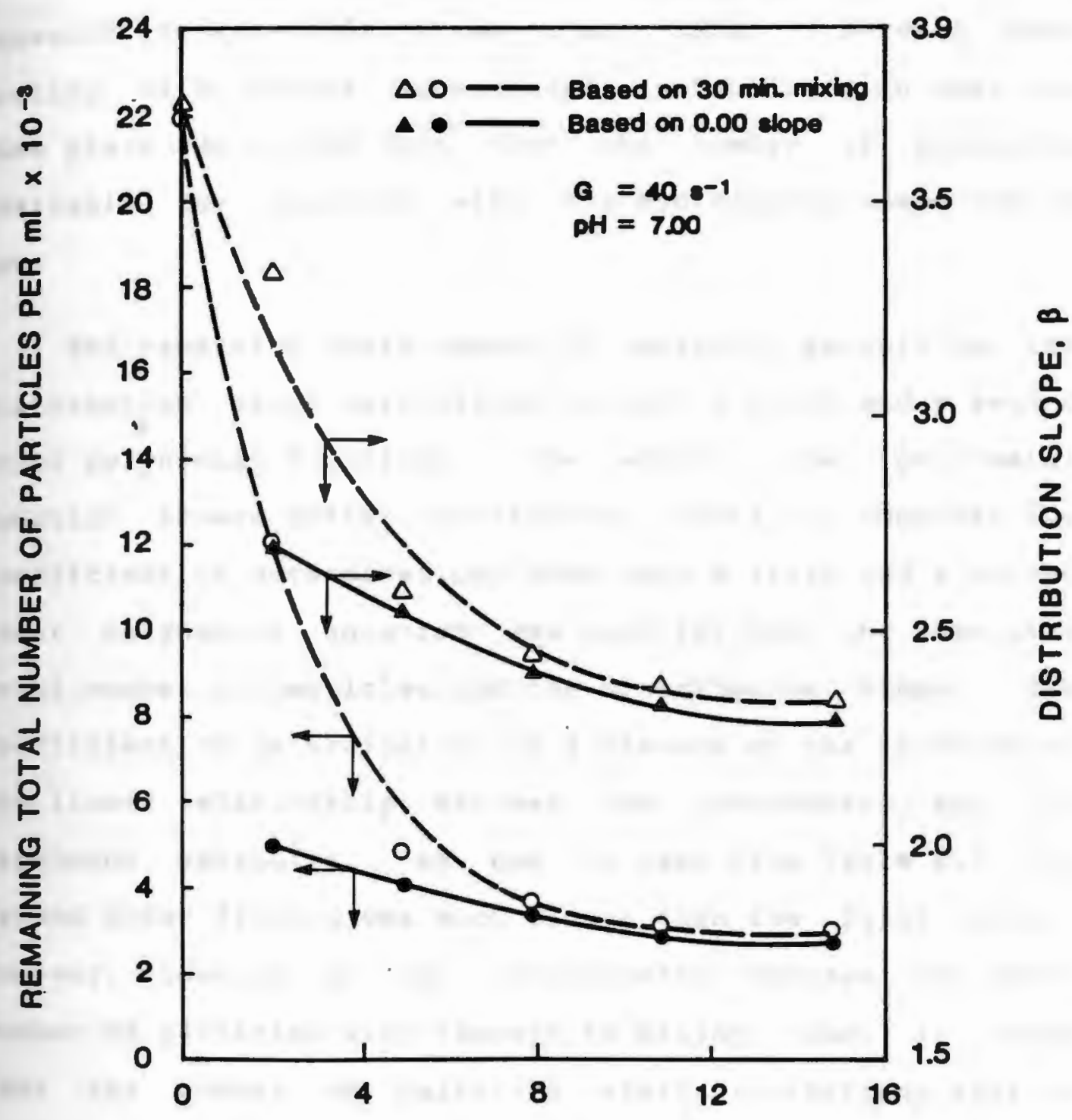

ALUM DOSE ( $\mathrm{mg} / \mathrm{L})$

Figure 4.3 Remaining total number of particles/ml and distribution slope versus coagulant dose at both 30 minutes mixing time and zero slope for jar test experiment number 1 . 
It was expected that restabilization would take place at the high alum dose (overdosing) as shown by previous research (Mackrle 1962; Stumm et al. 1968). However when dealing with dilute suspensions, restabilization does not take place due to the fact that the number of particles available for reaction with the hydrolyzing coagulant is low.

The remaining total number of particles as well as the distribution slope were fitted to both a first and a second order polynomial functions: The second order polynomial function showed better correlation. Table 4.1 compares the coefficient of determinations when both a first and a second order polynomial equation was used for both the remaining total number of particles and the distribution slope. The coefficient of determination is a measure of the strength of the linear relationship between the independent and the dependent variables. As can be seen from table 4.1, the second order fitting was much closer than the first order. However, looking at the relationship between the total number of particles with respect to mixing time, it seems that the number of particles starts to increase after a certain mixing time. This is shown in Table B-1 (Appendix B). This resulted in a closer curve fitting of a second order polynomial function. After a finite flocculation time, the maximum floc formation is reached and extended mixing will result only in the breakage of the flocs that have been previously formed. Prior to the optimum mixing 
Table 4.1- Coefficients of Determination Based on First and second order Polynomial Functions for Experiment Number 1

\begin{tabular}{|c|c|c|c|c|}
\hline $\begin{array}{l}\text { Coagulant } \\
\text { Dosage } \\
\text { (mg/L) }\end{array}$ & $\begin{array}{l}\text { First } \\
\text { distribution } \\
\text { slope }\end{array}$ & $\begin{array}{l}\text { Coefficient } \\
\text { order } \\
\text { remaining } \\
\text { particles }\end{array}$ & $\begin{array}{c}\text { of Determinat } \\
\text { second } \\
\text { distribution } \\
\text { slope }\end{array}$ & $\begin{array}{l}\text { ion } \\
\text { Order } \\
\text { remaining } \\
\text { particles }\end{array}$ \\
\hline 2 & 0.84 & 0.98 & 0.85 & 0.98 \\
\hline 5 & 0.70 & 0.81 & 0.91 & 0.93 \\
\hline 8 & 0.68 & 0.62 & 0.93 & 0.91 \\
\hline 11 & 0.64 & 0.66 & 0.96 & 0.90 \\
\hline 15 & , 0.61 & 0.55 & 0.90 & 0.87 \\
\hline
\end{tabular}

Table 4.2- Coefficients of Determination Based on First and second Order Polynomial Functions for Experiment Number 2

\begin{tabular}{ccccc}
$\begin{array}{c}\text { Coagulant } \\
\text { Dosage } \\
\text { (mg/L) }\end{array}$ & $\begin{array}{c}\text { First } \begin{array}{c}\text { Coefficient of } \\
\text { order } \\
\text { slope }\end{array} \\
\text { semaining } \\
\text { particles }\end{array}$ & $\begin{array}{c}\text { distribution } \\
\text { second order } \\
\text { slope }\end{array}$ & $\begin{array}{c}\text { oremaining } \\
\text { particles }\end{array}$ \\
\hline 2 & 0.71 & 0.89 & 0.75 & 0.99 \\
5 & 0.63 & 0.91 & 0.91 & 0.99 \\
8 & 0.81 & 0.86 & 0.98 & 0.99 \\
11 & 0.82 & 0.77 & 0.96 & 0.98 \\
\hline
\end{tabular}


time, the rate of flocculation exceeded the rate of floc breakage. When all particles were flocculated, then the rate of floc breakage was the dominant mechanism. The degree of floc breakage depends not only on the mixing time and intensity but also on factors such as the coagulant type and the nature of the particles present in the raw water. The flocs that are produced in water treatment are usually very soft and tend to break very easily. Their strength depends largely on the nature of the coagulant that is being used. Aluminum sulphate is known to produce soft flocs which have a low shear strength (Adin et al. 1974). The breakage occurs after the optimum mixing time is reached and it is more significant at the higher mixing intensities than at the low values. Therefore the data up to the optimum mixing time can be best fitted to a first order polynomial function, whereas after that time the data can be best fitted to a second order polynomial function. This will be discussed later when presenting the results obtained from the pilot plant mixing tank.

In the second experiment, the mixing intensity was reduced by half from $40 \mathrm{~s}^{-1}$ to $20 \mathrm{~s}^{-1}$ while all other parameters were held at the same values as in the first experiment. The results are shown in Figures 4.4 and 4.5 . In this case the rate of flocculation was lower than that which occurred at the higher mixing intensity, the optimum mixing time was reached at a later time, and floc breakage was not as significant as before. For 30 minutes mixing 


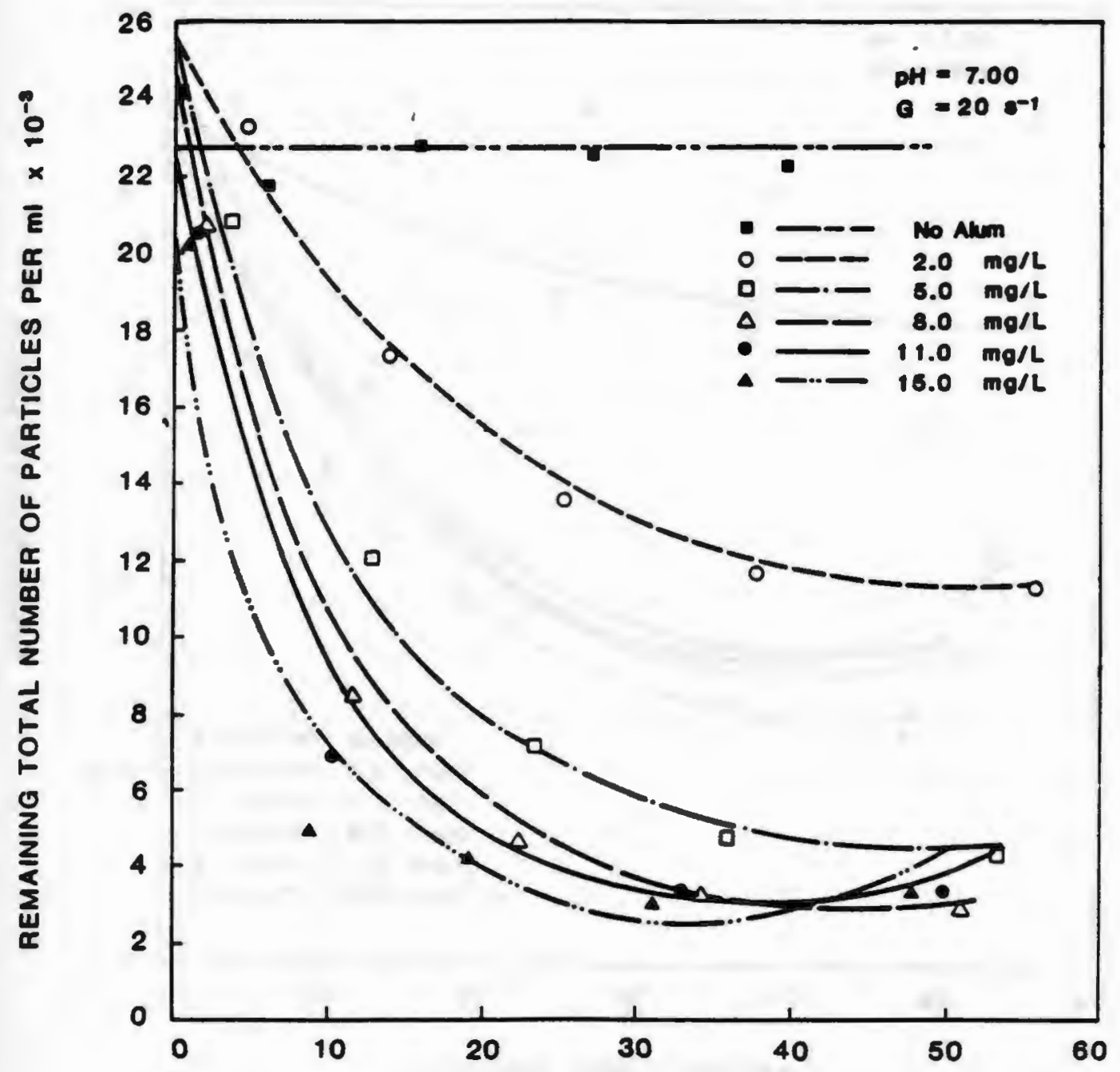

MIXING TIME ( minutes)

Figure 4.4 Remaining total number of particles/ml versus mixing time for jar test experiment number 2. 


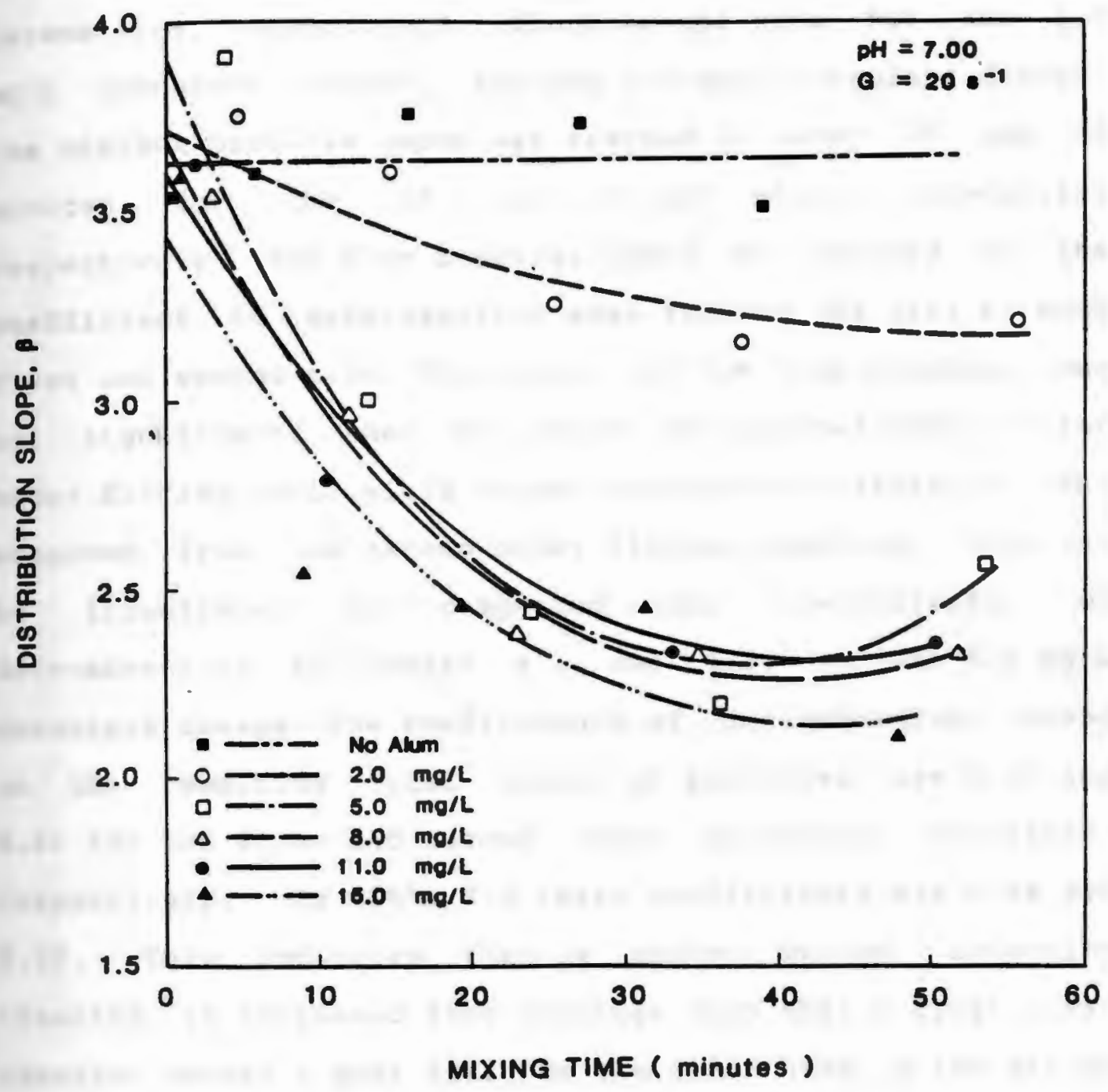

Figure 4.5 Distribution slope versus mixing time for jar test experiment number 2 . 
time, the remaining total number of particles was 12,900 and 12,000 particles/ml for the 20 and $40 \mathrm{~s}^{-1}$ mixing intensities, respectively. These counts were for the 2.0 $\mathrm{mg} / \mathrm{L}$ coagulant dosage. For the $8.0 \mathrm{mg} / \mathrm{L}$ coagulant dosage, the minimum particle count was reached at about 45 and 36 minutes for the 20 and $40 \mathrm{~s}^{-1}$ mixing intensities respectively. The floc breakage could be related to the coefficient of determination when fitting the data to both first and second order functions. If the floc breakage was not significant, then it would be expected that a first order fitting would yield higher correlation values to that obtained from the second order fitting equation. This can be illustrated by comparing the coefficients of determinations in Tables 4.1 and 4.2 . For the $8.0 \mathrm{mg} / \mathrm{L}$ coagulant dosage, the coefficients of determination (based on the remaining total number of particles) are 0.62 and 0.91 for the first and second order polynomial equations, respectively. In Table 4.2 these coefficients are 0.86 and 0.99. This indicates that a higher mixing intensity resulted in increased floc breakage such that a first order equation showed a poor fit. On the other hand, a low mixing intensity resulted in less floc breakage and this is reflected in the high value in the coefficient of determination for the first order equation.

Figure 4.6 was plotted the same way as Figure 4.3. As can be seen from the figure, the optimum alum dose is 8.0 $m g / L$ even at the low mixing intensity. However, in 


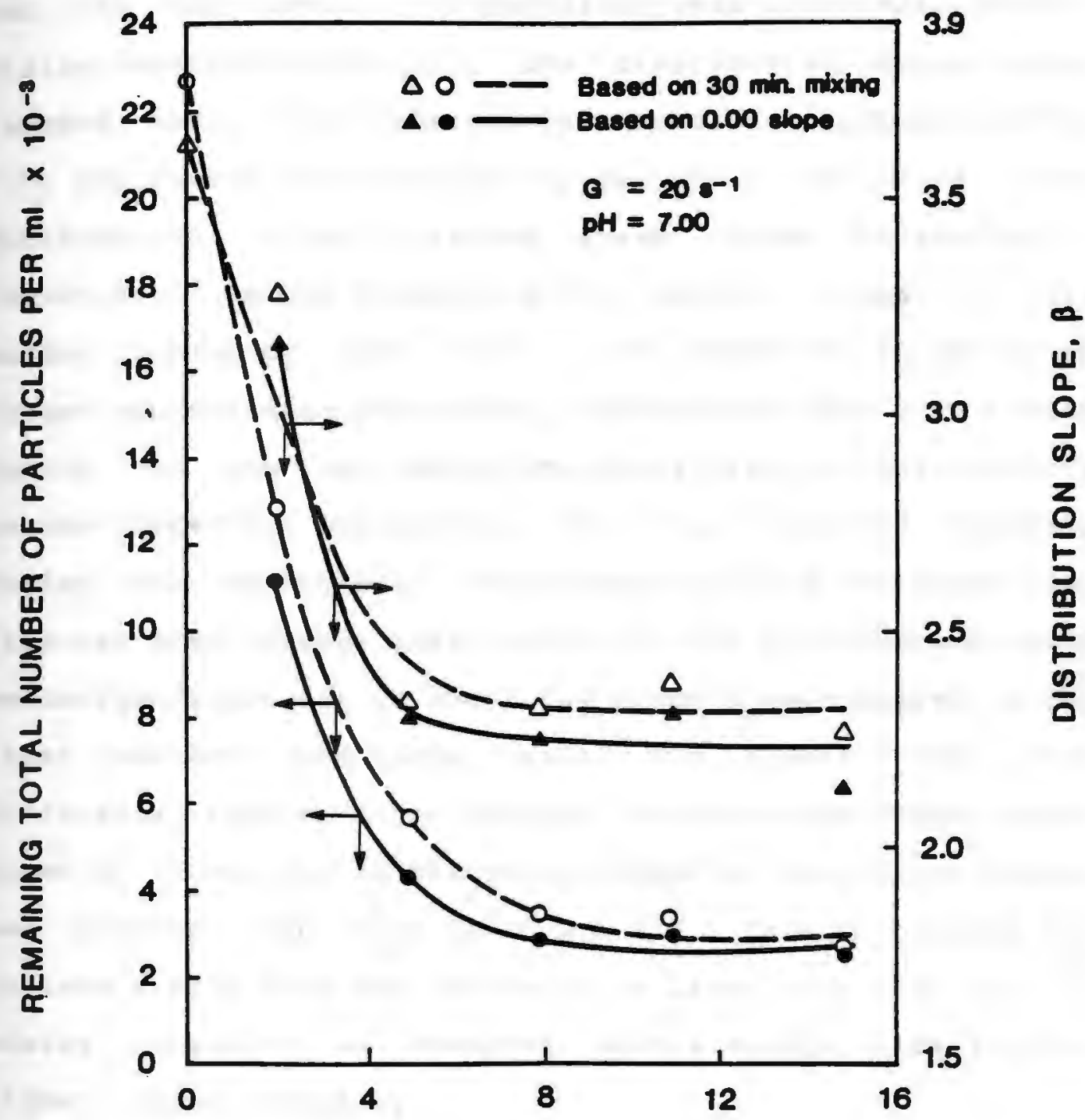

ALUM DOSE ( $\mathrm{mg} / \mathrm{L}$ )

Figure 4.6 Remaining total number of particles/ml and distribution slope versus coagulant dose at both 30 minutes mixing time and zero slope for jar test experiment number 2 . 
comparison to Figure 4.3 , the remaining total number of particles at the 30 minutes mixing time was almost the same, but the distribution slope values were different. with a nixing intensity of $20 \mathrm{~s}^{-1}$, the distribution slope value reached about 2.24 whereas at the mixing intensity of 40 $s^{-1}$, the lowest distribution slope value was 2.34 . The distribution slope reached lower values indicating a formation of larger flocs from the smaller flocs at this mixing intensity, and hence, the reduction in the total number of remaining particles. The maximum floc size formed during the run was above the upper limit of the particle counter detection capability. This was observed visually during the experiment. No attempt to find the large floc size was made because their effect on the distribution would be insignificant due to their few numbers as compared to the other smaller detectable sizes. In Figure 4.6, the difference between the minimum distribution slope values based on 30 minutes of mixing and based on zero slope values was greater than that in Figure 4.3. This is because the optimum mixing time was reached at a later time with the low mixing intensity as compared with a shorter time for the higher mixing intensity.

When the mixing intensity was increased to $65 \mathrm{~s}^{-1}$, the only significant change was in the rate of flocculation which was higher than in the previous cases. After 30 minutes of mixing, the remaining total number of particles were 3,300 and 5,760 particles/ml for the mixing intensities 
of 65 and $20 \mathrm{~s}^{-1}$, respectively. These values were for the $5.0 \mathrm{mg} / \mathrm{L}$ coagulant dosage. The optimum mixing time was shorter than that at the lower mixing intensities. For the $8.0 \mathrm{mg} / \mathrm{L}$ coagulant dosage, the zero slope for the remaining total number of particles was reached in 31 and 43 minutes for the mixing intensities of 65 and $20 \mathrm{~s}^{-1}$, respectively. For the $40 \mathrm{~s}^{-1}$ mixing intensity the zero slope was reached in 36 minutes. Figures 4.7 , and 4.8 are the plots of the remaining total number of particles and the distribution slope against mixing time. The best fit was found to be a second order polynomial equation as can be seen in Table 4.3 where the coefficients of determinations are listed for both first and second order fitting equations. Figure 4.9 was plotted the same way as Figures 4.3 and 4.6 which indicates that the optimum coagulant dosage is slightly below the 8.0 mg/L with this mixing intensity based on both the remaining total number of particles as well as the distribution slope.

From the above discussion it is clear that the mixing intensity has a direct effect on the rate of flocculation, the number of particles remaining, and on the distribution of the particles. This can be seen when comparing the results shown in Figures 4.3, 4.6, and 4.9. The distribution slope changed in the same way as the remaining number of particles. The distribution slope reached its minimum value at the same time as the total number of particles did. This indicates that the distribution of particles follows the same trend as the remaining number of 


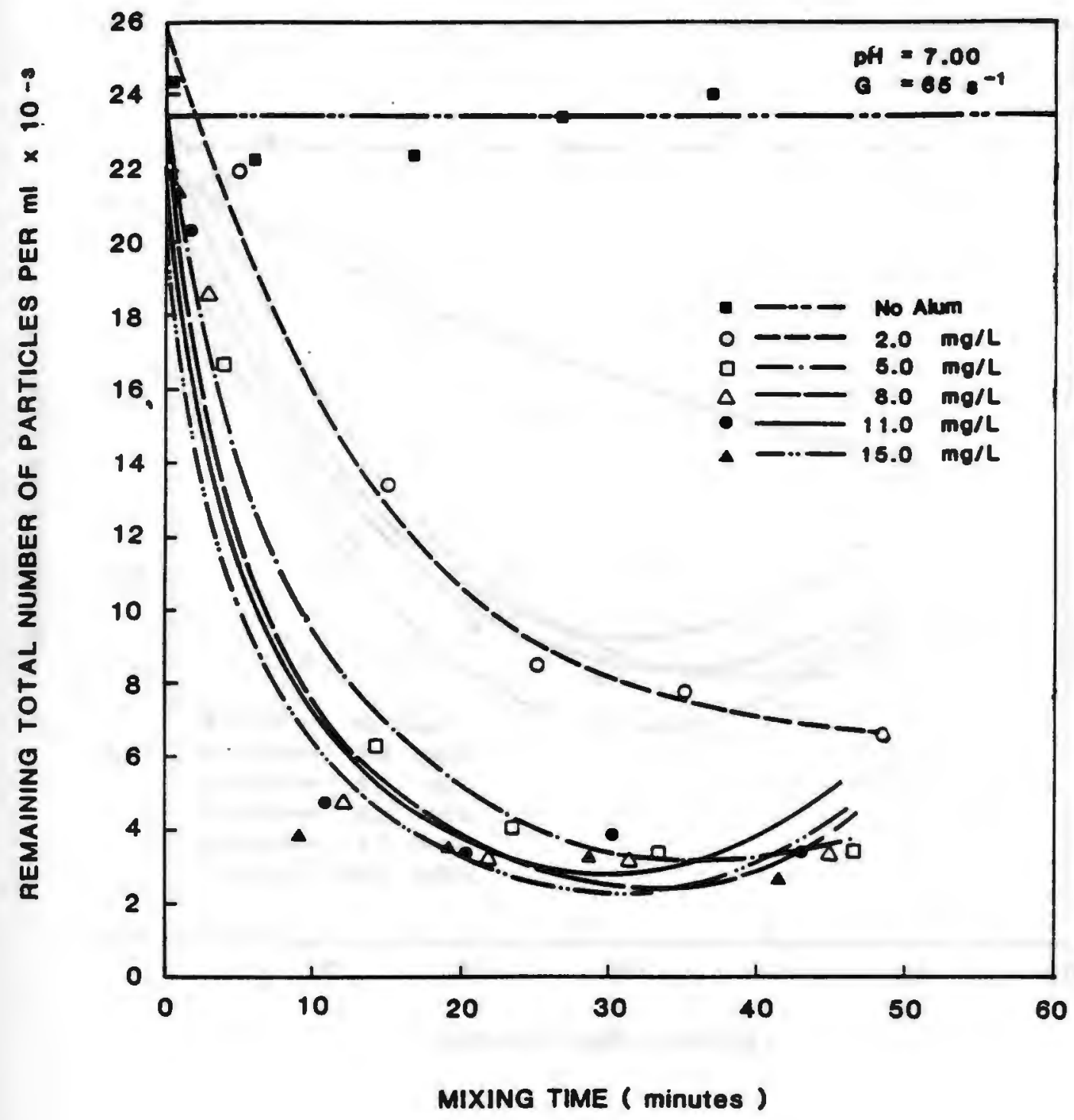

Figure 4.7 Remaining total number of particles/ml versus mixing time for jar test experiment number 3 . 


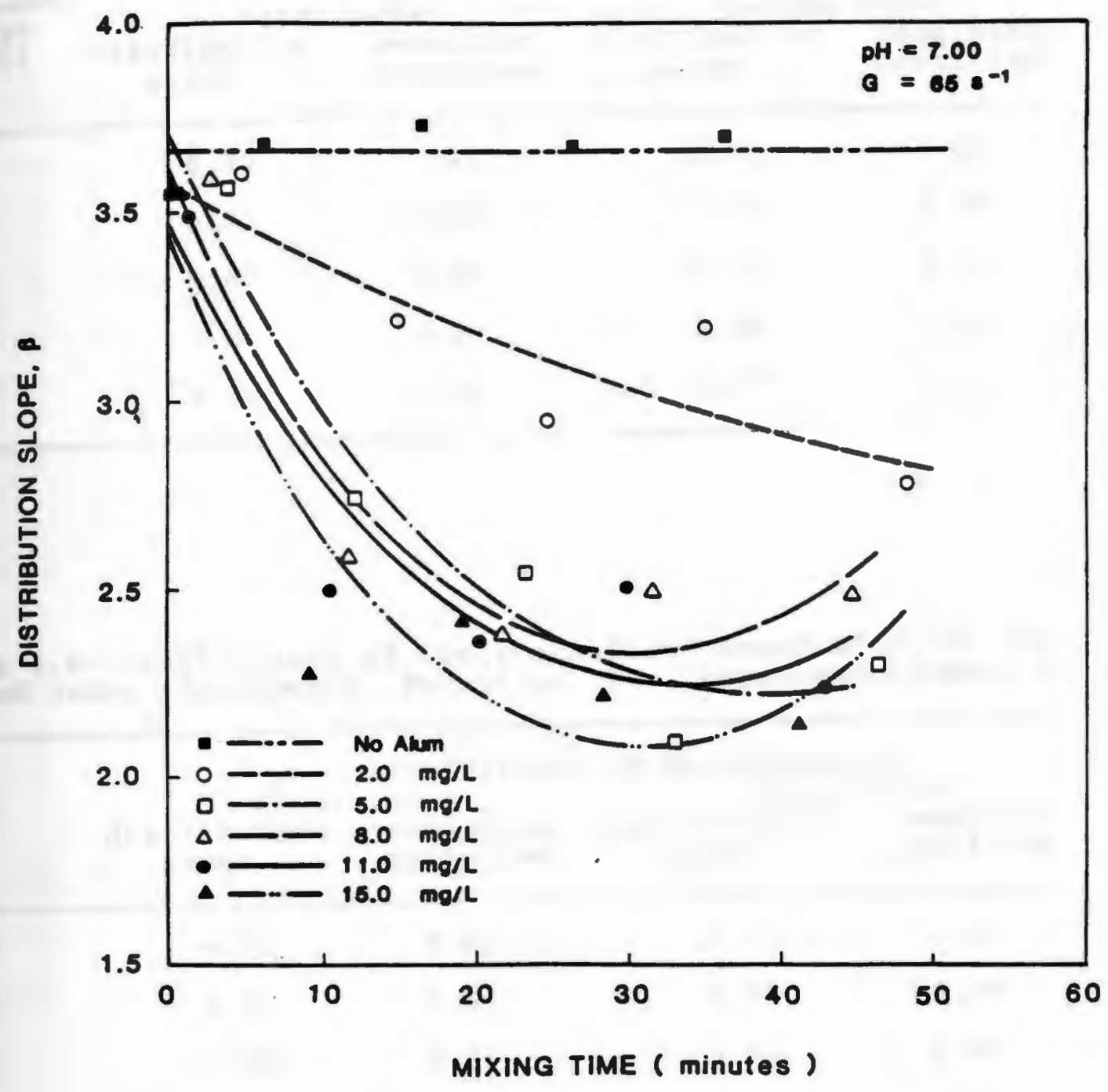

Figure 4.8 Distribution sloge versus mixing time for jar test experiment number 3 . 
Table 4.3- Coefficients of Determination Based on First and second Order Polynomial Functions for Experiment Number 3

\begin{tabular}{|c|c|c|c|c|}
\hline $\begin{array}{l}\text { Coagulant } \\
\text { Dosage } \\
(m g / L)\end{array}$ & $\begin{array}{l}\text { First } \\
\text { distribution } \\
\text { slope }\end{array}$ & $\begin{array}{l}\text { Coefficient } \\
\text { order } \\
\text { remaining } \\
\text { particles }\end{array}$ & $\begin{array}{c}\text { of Determinati } \\
\text { second } \\
\text { distribution } \\
\text { slope }\end{array}$ & $\begin{array}{l}\text { on } \\
\text { Order } \\
\text { remaining } \\
\text { particles }\end{array}$ \\
\hline 2 & 0.79 & 0.92 & 0.81 & 0.99 \\
\hline 5 & 0.82 & 0.80 & 0.95 & 0.99 \\
\hline 8 & 0.63 & 0.68 & 0.92 & 0.97 \\
\hline 11 & 0.91 & 0.67 & 0.88 & 0.94 \\
\hline 15 &,$\quad 0.68$ & 0.69 & 0.87 & 0.90 \\
\hline
\end{tabular}

Table 4.4- Coefficients of Determination Based on First and second order Polynomial Functions for Experiment Number 4

\begin{tabular}{ccccc}
\hline pH & $\begin{array}{c}\text { First } \\
\text { distribution } \\
\text { slope }\end{array}$ & $\begin{array}{c}\text { Coeficient of } \\
\text { remaining } \\
\text { particles }\end{array}$ & $\begin{array}{c}\text { dermination } \\
\text { distribution } \\
\text { slope }\end{array}$ & $\begin{array}{c}\text { order } \\
\text { remaining } \\
\text { particles }\end{array}$ \\
\hline 4 & 0.01 & 0.87 & 0.37 & 0.88 \\
5 & 0.01 & 0.98 & 0.75 & 0.98 \\
6 & 0.62 & 0.81 & 0.92 & 0.93 \\
7 & 0.70 & 0.57 & 0.90 & 0.93 \\
9 & 0.53 & 0.63 & 0.94 & 0.94 \\
\hline
\end{tabular}




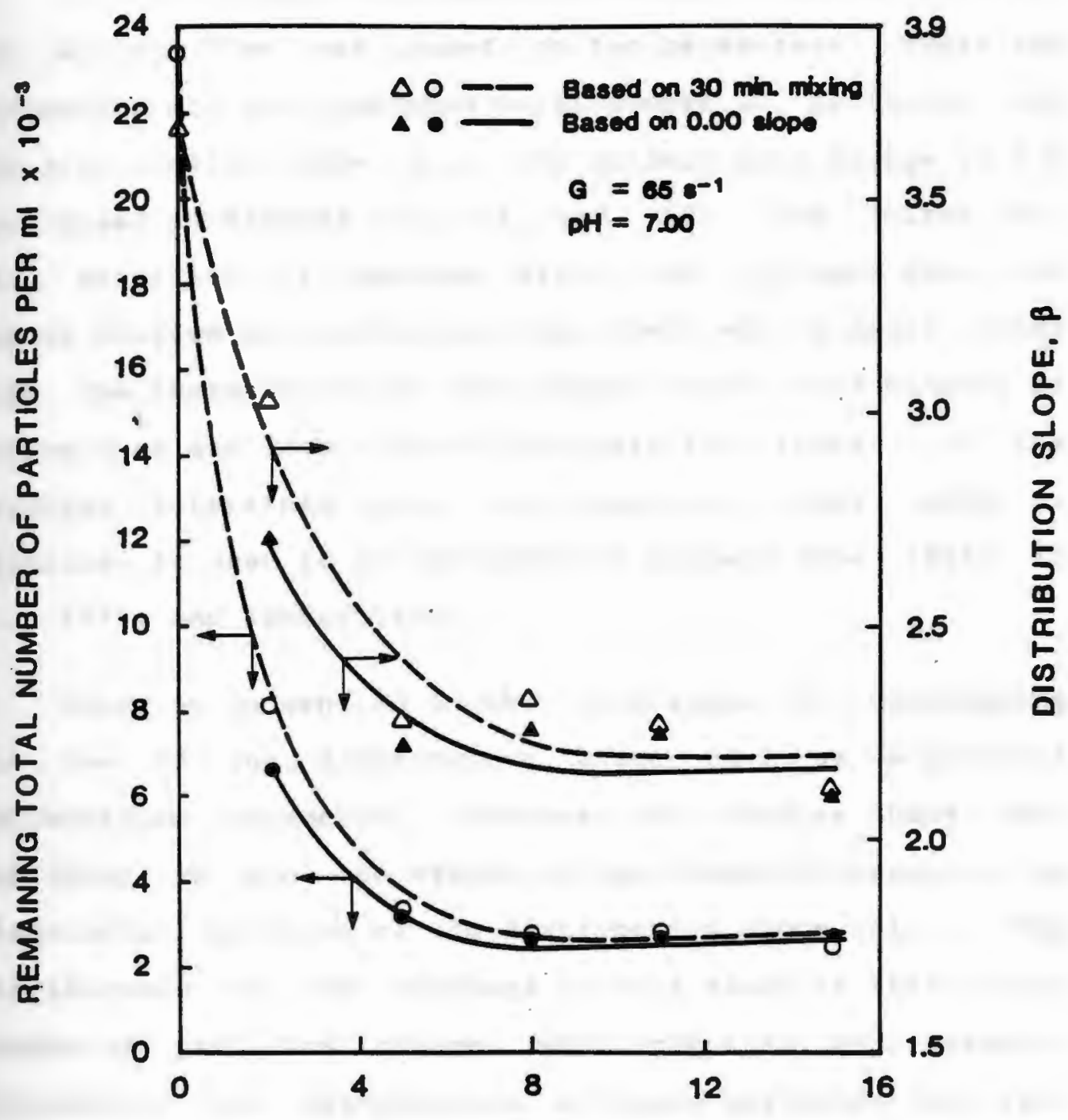

ALUM DOSE ( $\mathrm{mg} / \mathrm{L})$

Figure 4.9 Remaining total number of particles/ml and distribution slope versus coagulant dose at both 30 minutes mixing time and zero slope for jar test experiment number 3 . 
particles does.

In this study, the selection of the optimal alum dosage and mixing time was based on two parameters. These two parameters are the remaining total number of particles and the distribution slope $(\beta)$. The optimal alum dosage is 8.0 $m / L$ based on Figures 4.3, 4.6, and 4.9. The reason for this selection is because after the $8.0 \mathrm{mg} / \mathrm{L}$ dose, the curves flatten out indicating that there was no major change with the increase in the alum dosage based on 30 minutes of mixing time and zero slope of the best fit lines. In the existing literature only the remaining total number of particles is used as an indicator of optimum dose (Tate et al. 1977; and Tanaka 1986).

There is no mention in the literature of considering the use of the distribution slope $(\beta)$ as a possible optimization parameter. Moreover no studies have been published to show the effect of the chemical dosage on the distribution in terms of the distribution slope value. The significance of the findings in this study is that as the number of particles changes with physical and chemical parameters, the distribution of these particles will also change in the same direction. Underdosing will cause a slight change in the remaining particles as well as the distribution slope value which is an indicator of the change in the distribution of the particles. Another finding is the fact that the flocculation of particles was not a first but a second order reaction when the mixing time was 
extended beyond the optimum.

The last three experiments were performed to study the effect of the $\mathrm{pH}$ on the particle size distribution. For experiment number 4 , the mixing intensity was kept at 40 $\mathrm{s}^{-1}$, and the alum dose used was $8.0 \mathrm{mg} / \mathrm{L}$. The results are shown in Figures 4.10 , and 4.11 . In Figure 4.10 it is clear that the total number of particles went down with time and the rate of flocculation increased with an increase in the pH. Based on Figure 4.10 , the rate of flocculation increased from 149 to 681 particles/ml-min when the pH was increased from 4.00 to 9.00 based on 30 minutes of mixing time.

At $\mathrm{pH} 4.00$ the rate of flocculation was very low. This is because at this pa most of the alum is in the soluble form as shown by the solubility curves of aluminum sulphate by O'Melia (1969), O'Melia (1972), Benefield et al. (1982), Edwards et al. (1985) and AWWA (1971). When aluminum (III) salt is added to water in concentrations less than the solubility limit of the metal hydroxide, the hydrolysis products will form and adsorb onto the particles, causing destabilization by charge neutralization only. When the metal salt concentration is in excess of the solubility of the metal hydroxide, a metal hydroxide precipitate is formed, and both charge neutralization and enmeshment in the precipitate contribute to coagulation. At low $\mathrm{pH}$ values, most of the alum is in the soluble form which results in less coagulation effectiveness. 


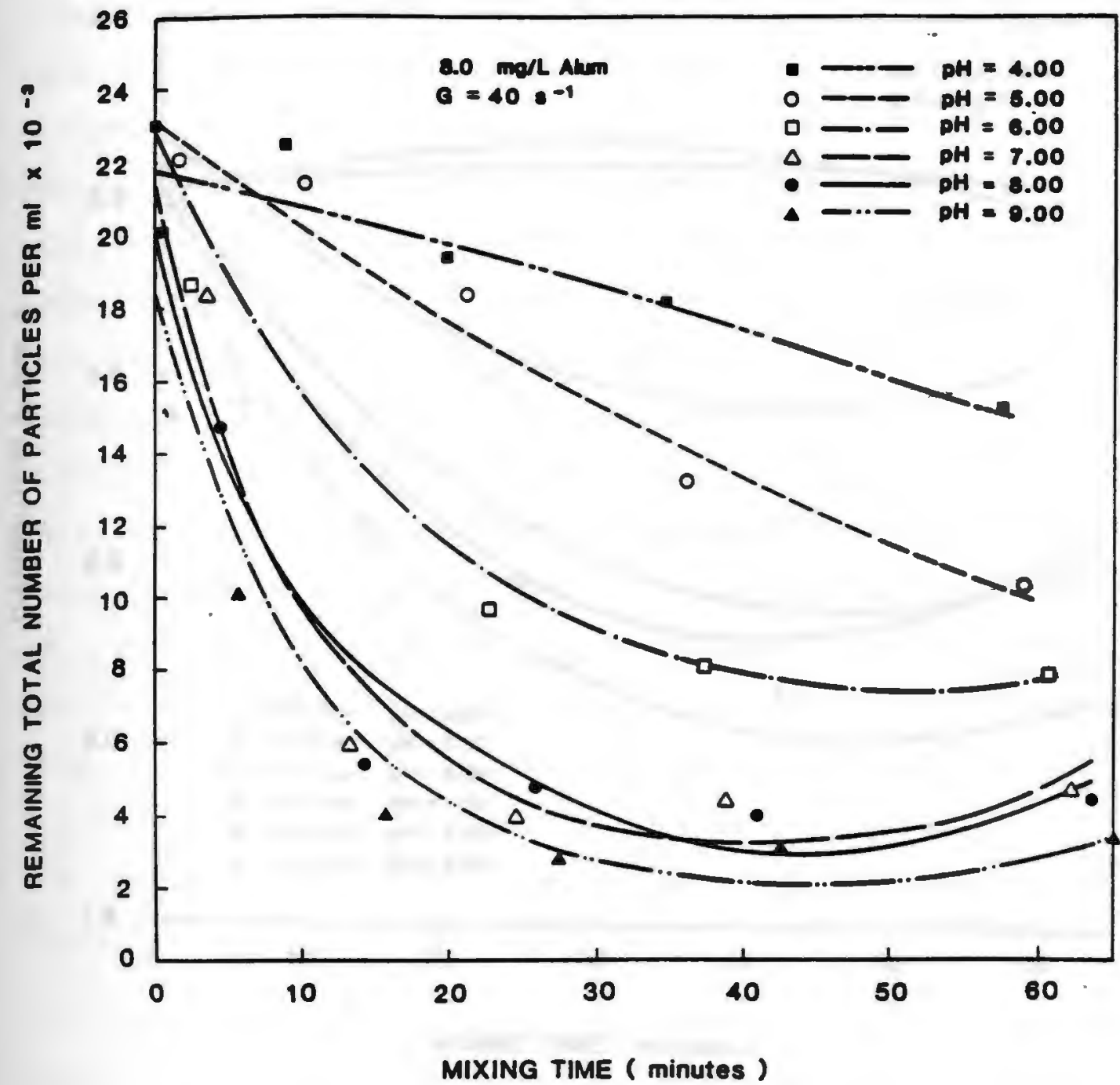

Pigure 4.10 Remaining total number of particles/ml versus mixing time for far test experiment number 4. 


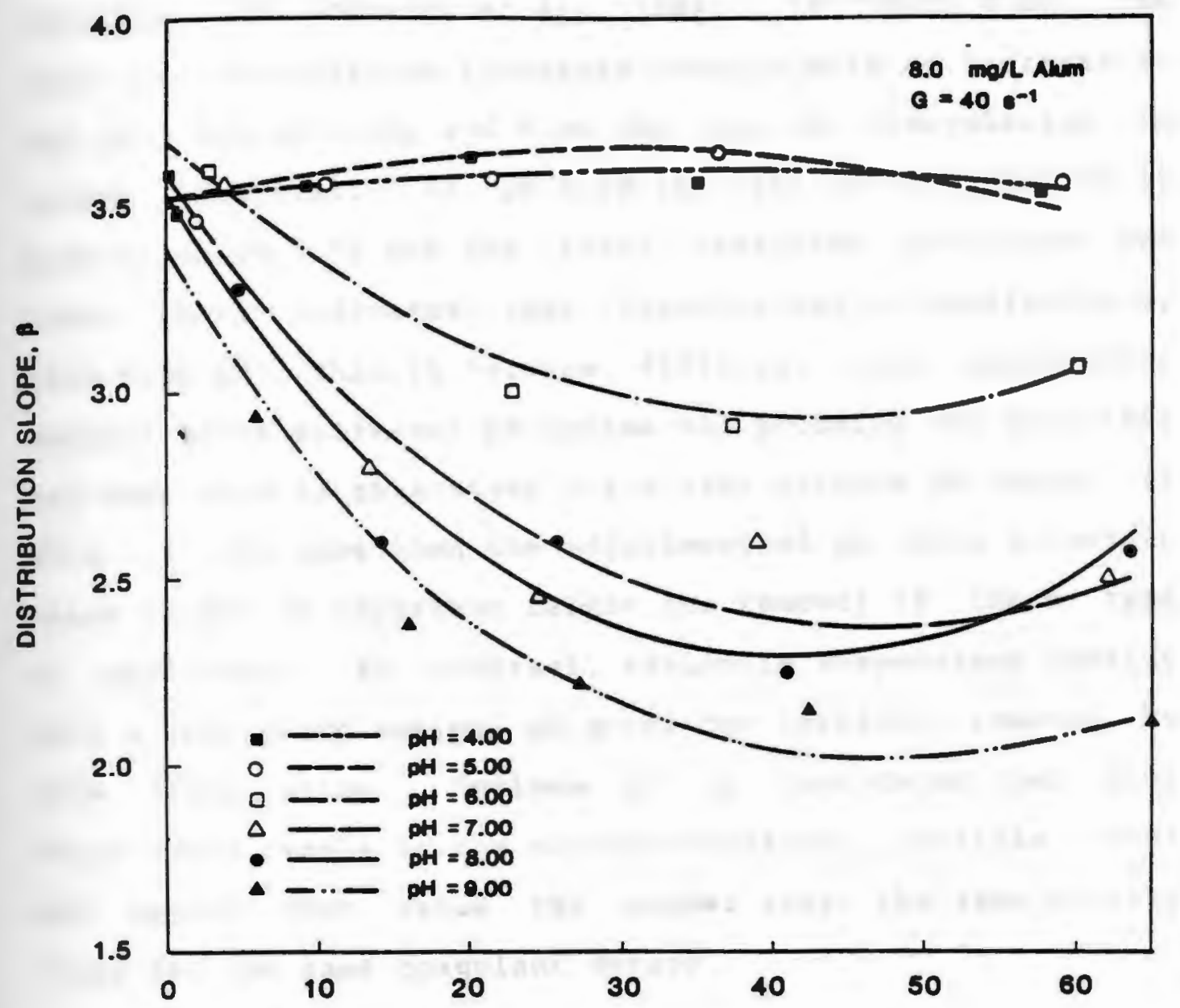

MIXING TIME (minutes)

Figure 4.11 Distribution slope versus mixing time for jar test experiment number 4 . 
In practice, the optimum pH range for the removal of turbidity by alum flocculation is generally 6.00-7.80 (Rawamura 1976, Edwards et al. 1985). In Figure 4.10, the rate of flocculation increases sharply with an increase in the pH. For pH 7.00 , and 8.00 the rate of flocculation is almost identical. At pH 9.00 the rate of flocculation is similar to pH 7.00 but the total remaining particles are lower which indicates that flocculation is continuing at this high pH. This is because different clay suspensions exhibit quite different $\mathrm{pH}$ optima and probably the clay that has been used in this study has a wide optimum ph range. If this is the case then the adjustment of $\mathrm{pH}$ above a certain value is not an important factor for removal of these type of particles. In contrast, kaolinite suspensions usually have a very sharp optimal pH point for turbidity removal by alum flocculation. Optimum $\mathrm{pH}$ is considered that value which would result in the minimum remaining particle count and beyond that value the number stays the same or very close for the same coagulant dosage.

In Figure 4.11 the slope of the distribution function stayed relatively constant for $\mathrm{pH} 4.00$ and 5.00 . However, the total number of particles for these two pH values declined, as can be seen in Figure 4.10, indicating the existence of some kind of flocculation. One explanation for this discrepancy is the possibility that the rate of flocculation is the same as the rate at which the submicron particles (not measured)are transferred to larger sizes, 
therefore the whole curve is shifted downward, keeping the distribution the same but with a decrease in the total remaining particles. Since the particle counter does not detect below the 2 micron size, then it would be difficult to verify this conclusion. visual observation during the course of the experiment indicated no change in the size of particles at these two pH values as compared to the other four. In Table B-4, Appendix $B$ it is clear that the number of particles below the 12.5 micron size decreased and above this size the number increased with time at pH 4.00 . However, at pH 5.00, there is no clear pattern of the change in the number of particles with size and time.

Figure 4.12 is a plot of the remaining total number of particles and the distribution slope values versus pt for 30 minutes mixing time and zero slope of the curves in Figures 4.10 and 4.11 . The figure shows that at low pH values 14.00 and 5.00) the remaining total number of particles decreased as the $\mathrm{pH}$ was increased but the distribution slope value started declining after a pH value of 5.00. As was mentioned before, the possible cause for this disagreement is the submicron particles and the rate of flocculation for the micron sized particles. The change in the remaining numbers and the distribution slope value during the time interval between the 30 minutes mixing time and the time needed to reach zero slope of the curves is shown in Figures 4.10 and 4.11 . As the $\mathrm{pH}$ increased, the gap between the two curves for the remaining total number of particles in Figure 


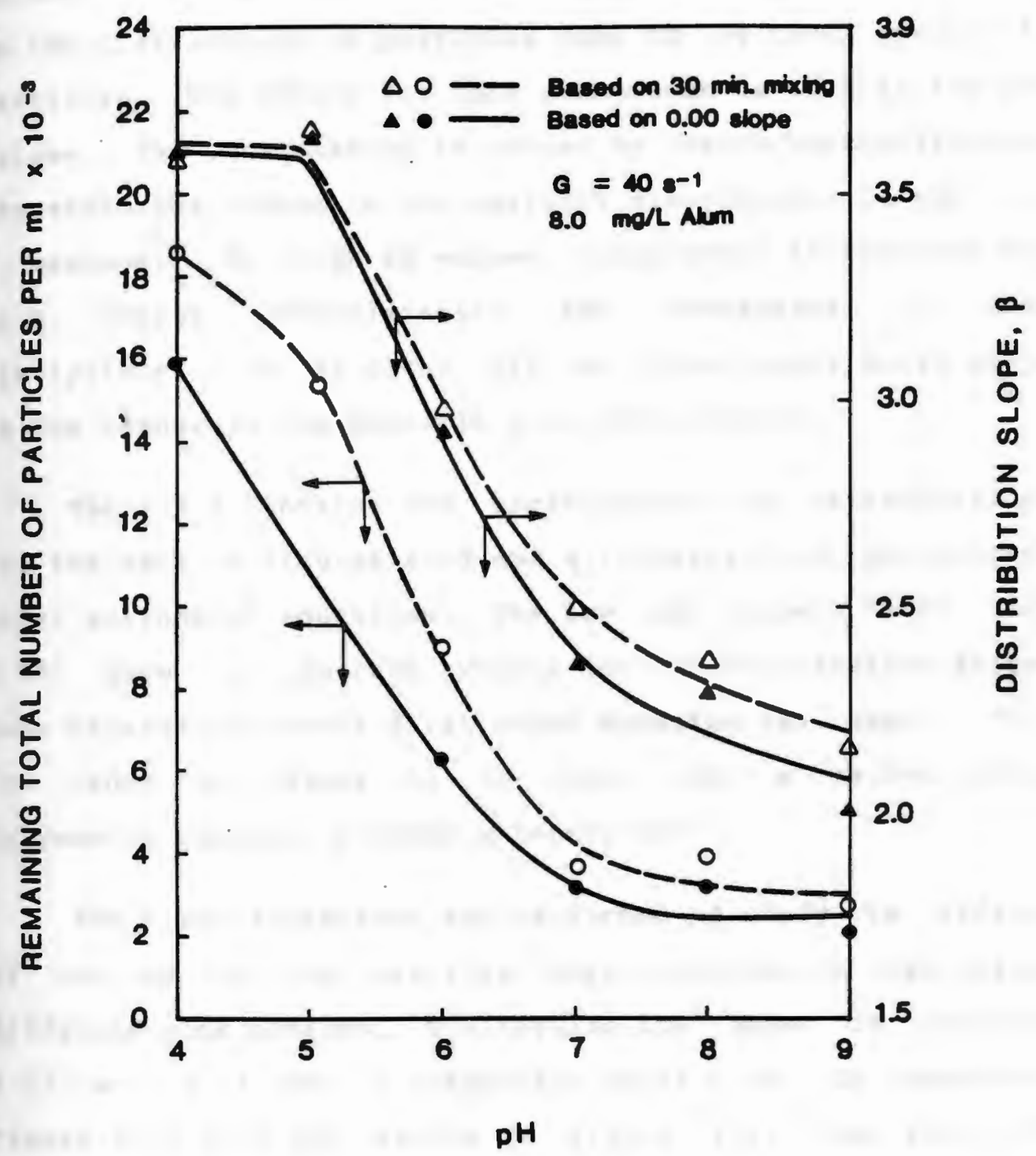

Figure 4.12 Remaining total number of particles/ml and distribution slope versus solution pli at both 30 minutes mixing time and zero slope for jar test experiment number 4. 
4.12 decreased but for the distribution slope, the gap increased. This indicates that the pH has a greater impact on the distribution of particles than on the total number of particles. The reason for this phenomenon is that at low pH values, the coagulation is caused by charge neutralization therefore the change in the particle distribution is not at a maximum. At high pi values, coagulation is achieved by both charge neutralization and enmeshment in the precipitate. It is clear that the latter contributes more to the change in the particle size distribution.

Table 4.4 contains the coefficients of determination for the data in Figures 4.10 and 4.11 using first and second order polynomial equations. The low $\mathrm{pH}$ values $(4.00$ and 5.00) gave the poorest values for the distribution slope data especially when a first order equation was used. For the other $\mathrm{pH}$ values it is clear that a second order polynomial equation yielded a better fit.

The fifth experiment was performed to study the effect of low $\mathrm{pH}$ on the particle size distribution when using different alum dosages. The results are shown in Figures 4.13 and 4.14 for a suspension pH of 6.00. In comparing Figure 4.13 with the results in Figure 4.1 , the rate of flocculation in the former figure at different dosages is low and the time required to reach the minimum particle count is high. With $8 \mathrm{mg} / \mathrm{L}$ of coagulant, the remaining total particles were 5,948 and 3,529 particle/ml for pH 6.00 and 7.00 respectively based on the zero slope values. The 


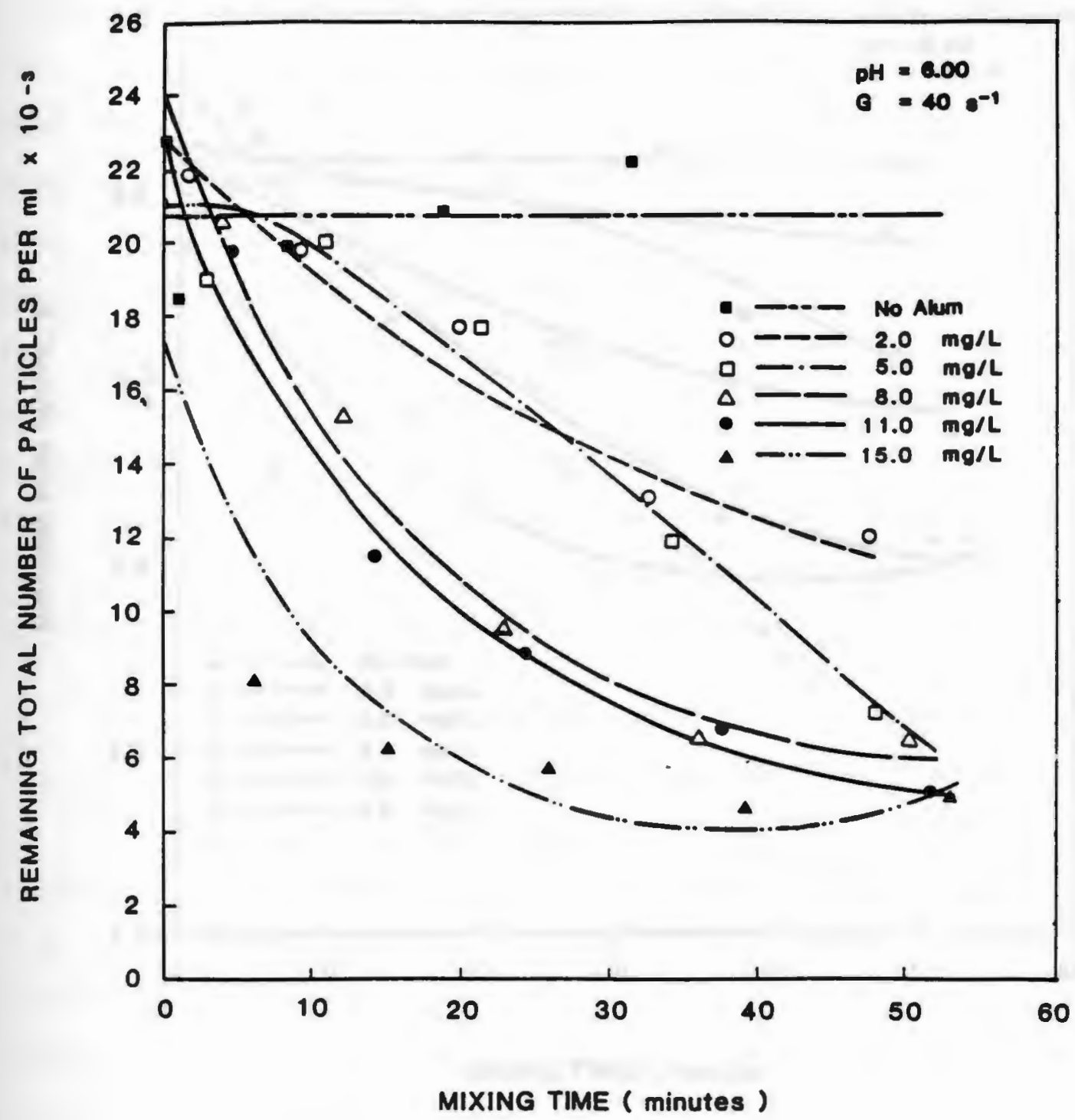

Figure 4.13 Remaining total number of particles/ml versus mixing time for jar test experiment number 5 . 


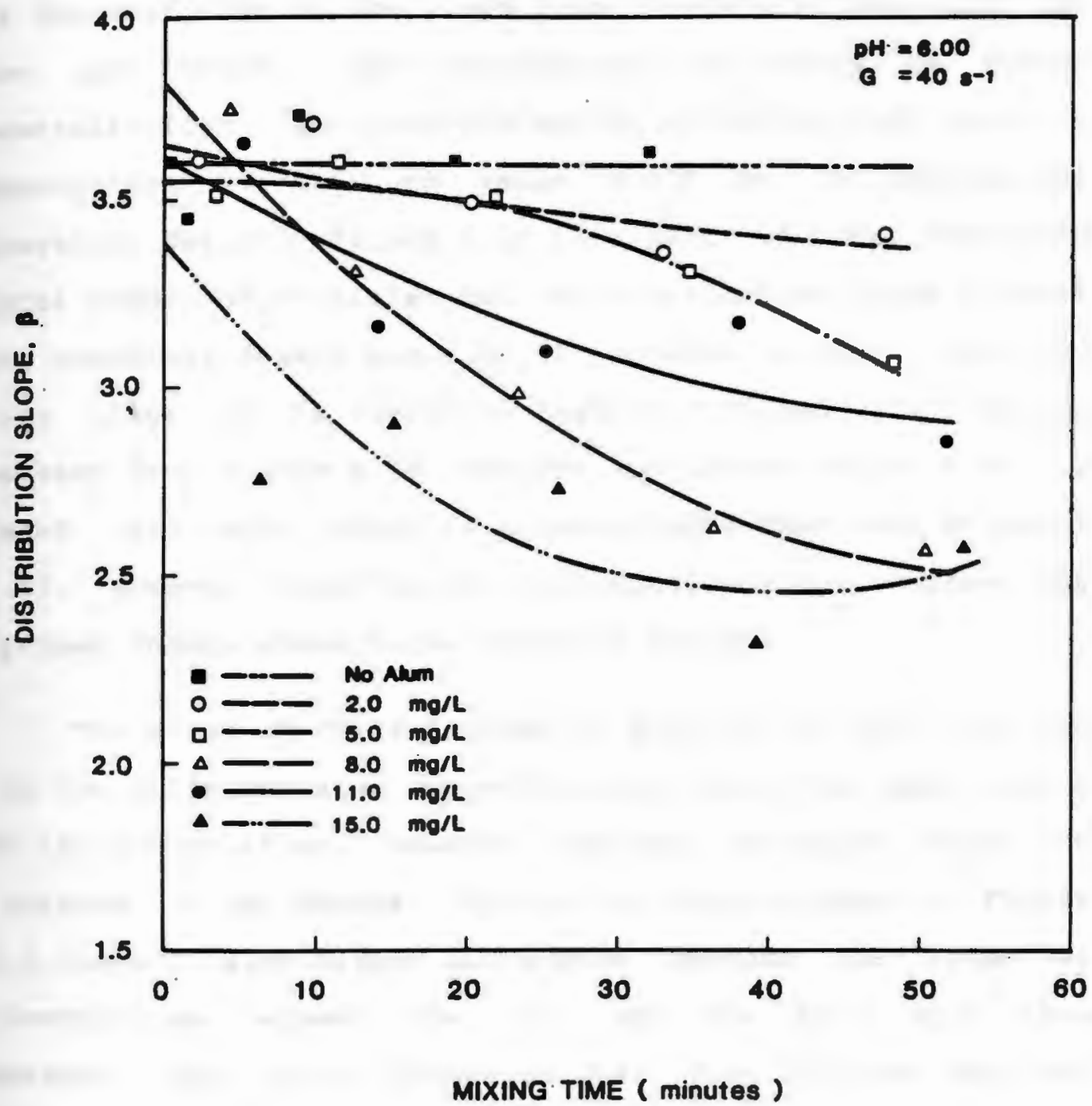

Figure 4.14 Distribution slope versus mixing time for jar test experiment number 5 . 
time to reach those counts was 55 and 36 minutes for $\mathrm{pH} 6.00$ and 7.00 respectively. The reason for this low flocculation is the fact that at the given alum concentrations and the low pt value, the coagulation is mostly by charge neutralization. One possible way of achieving high particle coagulation at this pH value would be to increase the coagulant dosage. Figure 4.15 is a plot of the remaining total number of particles and the distribution slope against the coagulant dosage based on 30 minutes mixing time and zero slope of the curves in Figures 4.13 and 4.14. As can be seen from Figure 4.15, the optimum dosage at pH 6.00 is about $15.0 \mathrm{mg} / \mathrm{L}$ which is almost double than that at $\mathrm{pH}$ of 7.00. However, based on the distribution slope values the optimum dosage seems to be about $12.0 \mathrm{mg} / \mathrm{L}$.

The slope of the distribution function at this low pH for the different alum concentrations shows the same results of low flocculation. However, the rate increases with the increase in the dosage. Unlike the results shown in Figure 4.2 there is significant difference between the rates of flocculation between the 11.0 and the $15.0 \mathrm{mg} / \mathrm{L}$ alum dosages. This is an indication that flocculation has not reached a maximum value at this $\mathrm{pH}$ and low alum concentration. The reason that the flocculation rate is high at the $15.0 \mathrm{mg} / \mathrm{L}$ alum is the fact that at this dose and $\mathrm{pH}$, the coagulation is achieved by both charge neutralization and enmeshment. 


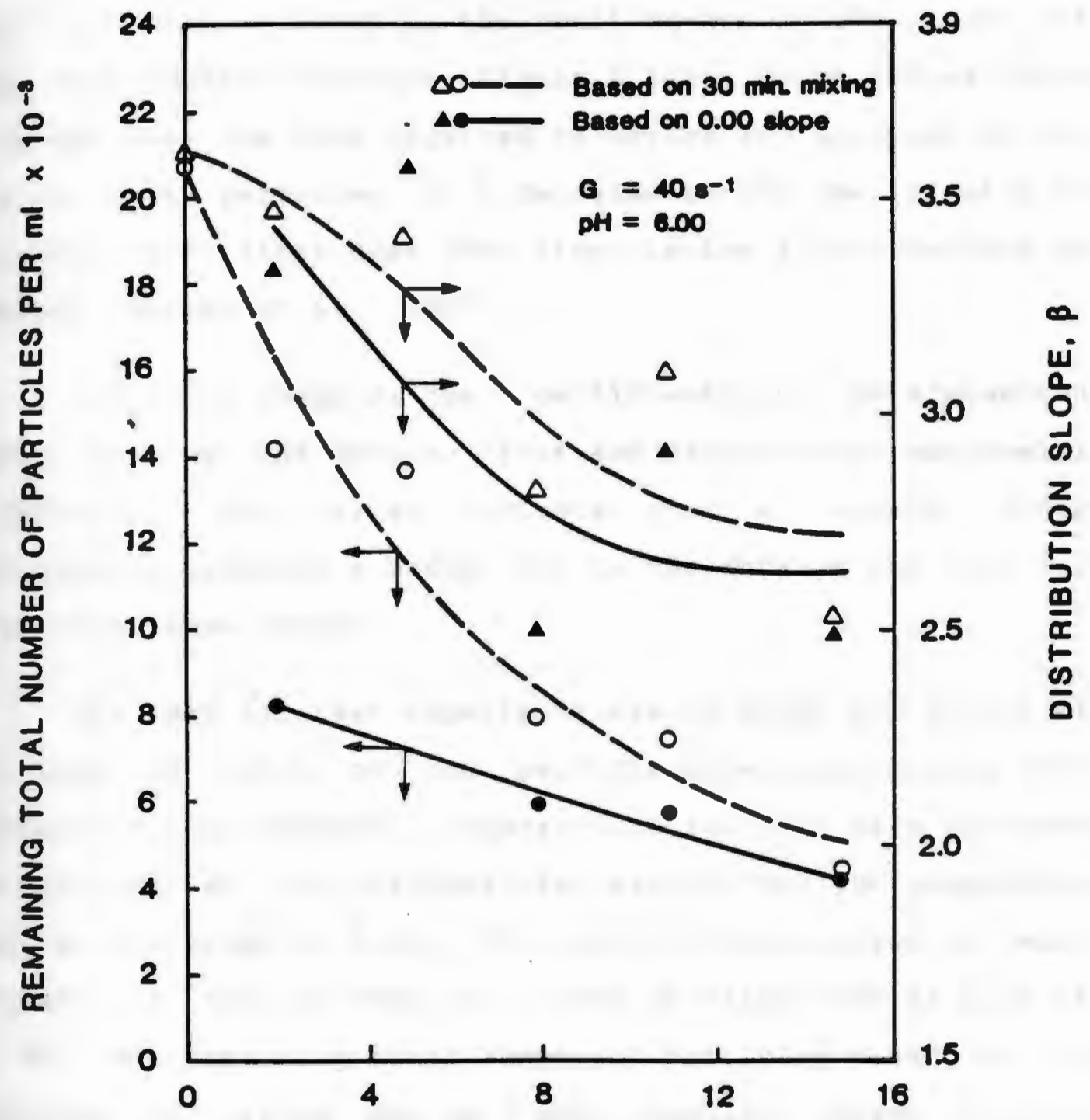

ALUM DOSE ( $\mathrm{mg} / \mathrm{L})$

Figure 4.15 Remaining total number of particles/ml and distribution slope versus coagulant dose at both 30 minutes mixing time and zero slope for jar test experiment number 5 . 
The time required to cause any change in the number of particles of any certain size is much longer at pH 6.0 than at 7.0. This is shown by the small change in the slope of the distribution function (Figure 4.14). At pH values below the optimum, the time required to notice any increase in the large size particles or a decrease in the smaller size is usually three times that when flocculating at the optimum pH values (Hannah et al. 1967).

Table 4.5 compares the coefficients of determination when fitting the data to first and second order polynomial equations. The values indicate that a second order polynomial provides a better fit to the data as was true for the other experiments.

The last jar test experiment was to study the effect of a high pH value on the particle size distribution with different alum dosages. Figures 4.16 and 4.17 have the same conditions as the previous case except that the suspension pH was increased to 8.00 . The rate of flocculation is much higher at this pH than at a lower pH value even at a pH of 7.00. The remaining total number of particles based on 30 minutes of mixing and an $8 \mathrm{mg} / \mathrm{L}$ coagulant dosage is 7,890 and 3,308 particles/ml for the $\mathrm{pH}$ of 6.00 and 8.00 respectively. The minimum total number of particles was identical for both 5.0 and $8.0 \mathrm{mg} / \mathrm{L}$ alum dosages indicating that at this high pH value a lower alum dose was sufficient to bring about the maximum particle destabilization. No major change occurred after the $5.0 \mathrm{mg} / \mathrm{L}$ coagulant dosage in 
Table 4.5- Coefficients of Determination Based on First and second order Polynomial Functions for Experiment Number 5

\begin{tabular}{ccccc}
$\begin{array}{c}\text { Coagulant } \\
\text { Dosage } \\
(\text { ag/L) }\end{array}$ & $\begin{array}{c}\text { First } \begin{array}{c}\text { Coefficient of Determination } \\
\text { order } \\
\text { slope }\end{array} \\
\text { semaining } \\
\text { particles }\end{array}$ & $\begin{array}{c}\text { distribution } \\
\text { order } \\
\text { slope }\end{array}$ & $\begin{array}{c}\text { remaining } \\
\text { particles }\end{array}$ \\
\hline 2 & 0.66 & 0.97 & 0.67 & 0.98 \\
5 & 0.86 & 0.91 & 0.98 & 0.98 \\
8 & 0.91 & 0.94 & 0.93 & 0.99 \\
11 & 0.79 & 0.95 & 0.81 & 0.97 \\
15 & 0.58 & 0.60 & 0.74 & 0.87 \\
\hline
\end{tabular}

Table 4.6- Coefficients of Determination Based on First and second Order Polynomial Functions for Experiment Number 6

\begin{tabular}{|c|c|c|c|c|}
\hline $\begin{array}{l}\text { Coagulant } \\
\text { Dosage } \\
(\mathrm{mg} / \mathrm{L})\end{array}$ & $\begin{array}{c}\text { First } \\
\text { distribution } \\
\text { slope }\end{array}$ & $\begin{array}{l}\text { Coefficient } \\
\text { order } \\
\text { remaining } \\
\text { particles }\end{array}$ & $\begin{array}{c}\text { of Determinati } \\
\text { second } \\
\text { distribution } \\
\text { slope }\end{array}$ & $\begin{array}{l}\text { on } \\
\text { Order } \\
\text { remaining } \\
\text { particles }\end{array}$ \\
\hline 2 & 0.88 & 0.87 & 0.95 & 0.99 \\
\hline 5 & 0.81 & 0.89 & 0.98 & 0.99 \\
\hline 8 & 0.75 & 0.73 & 0.93 & 0.95 \\
\hline 11 & 0.61 & 0.69 & 0.96 & 0.98 \\
\hline 15 & 0.79 & 0.62 & 0.88 & 0.94 \\
\hline
\end{tabular}




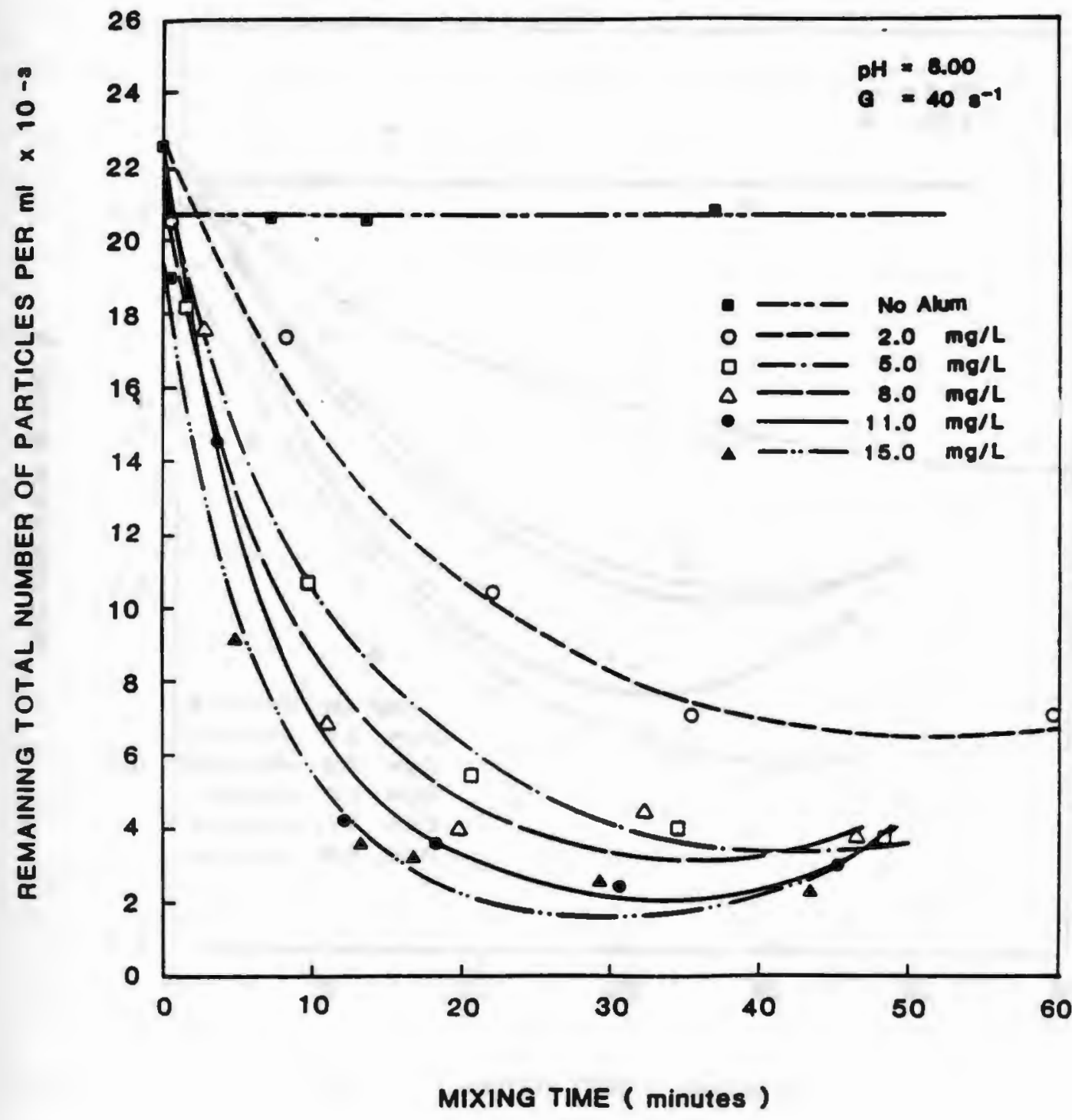

Figure 4.16 Remaining total number of particles/ml versus mixing time for jar test experiment number 6 . 


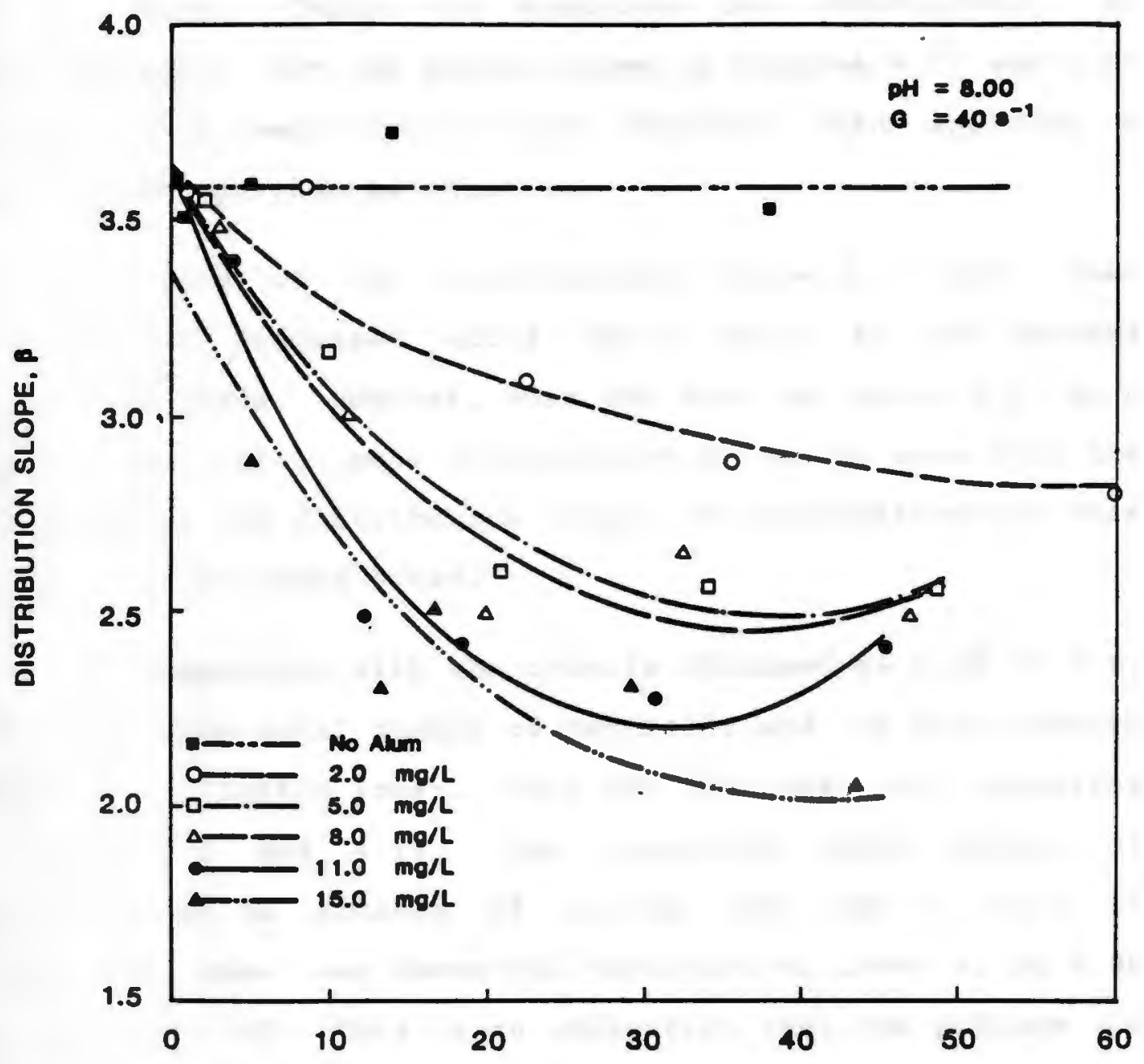

MIXING TIME ( minutes)

Figure 4.17 Distribution slope versus mixing time for jar test experiment number 6 . 
terms of the remaining total number of particles. This is shown in Figure 4.18 which was plotted the same way as Figure 4.15. Table 4.6 contains the coefficients of determination for the curves shown in Figures 4.16 and 4.17 along with a comparison to those obtained when applying a first order polynomial equation.

In term of the distribution slope, $\beta$, the same conclustons discussed above would apply to the dosages mentioned above. However, when the dose was above $8.0 \mathrm{mg} / \mathrm{L}$ there was still more flocculation as can be seen from the decrease in the distribution slope. No explanation for this result has yet been given.

In comparison with the results obtained at a pH of 7.0 , the remaining total number of particles and the distribution slope are slightly lower. This can be seen by comparing Figures 4.3 and 4.18 . The remaining total number of particles at 30 minutes of mixing time and $5 \mathrm{mg} / \mathrm{L}$ of coagulant dose was about 900 particles/ml lower at pH 8.00 than at $\mathrm{pH} 7.00$. This is an indication that the optimum pH for this kind of suspension is above 7.0. Different waters have different optimum $\mathrm{pH}$ for best particle removal. Rawamura (1976) investigated three types of water and concluded that different clay suspensions exhibit quite different pH optima. The three waters were a natural river water, a bentonite suspension synthetic water, and a kaolinite suspension synthetic water. The bentonite clay had a wide optimum $\mathrm{pH}$ range of 4 to about 6.2 , the kaolinite 


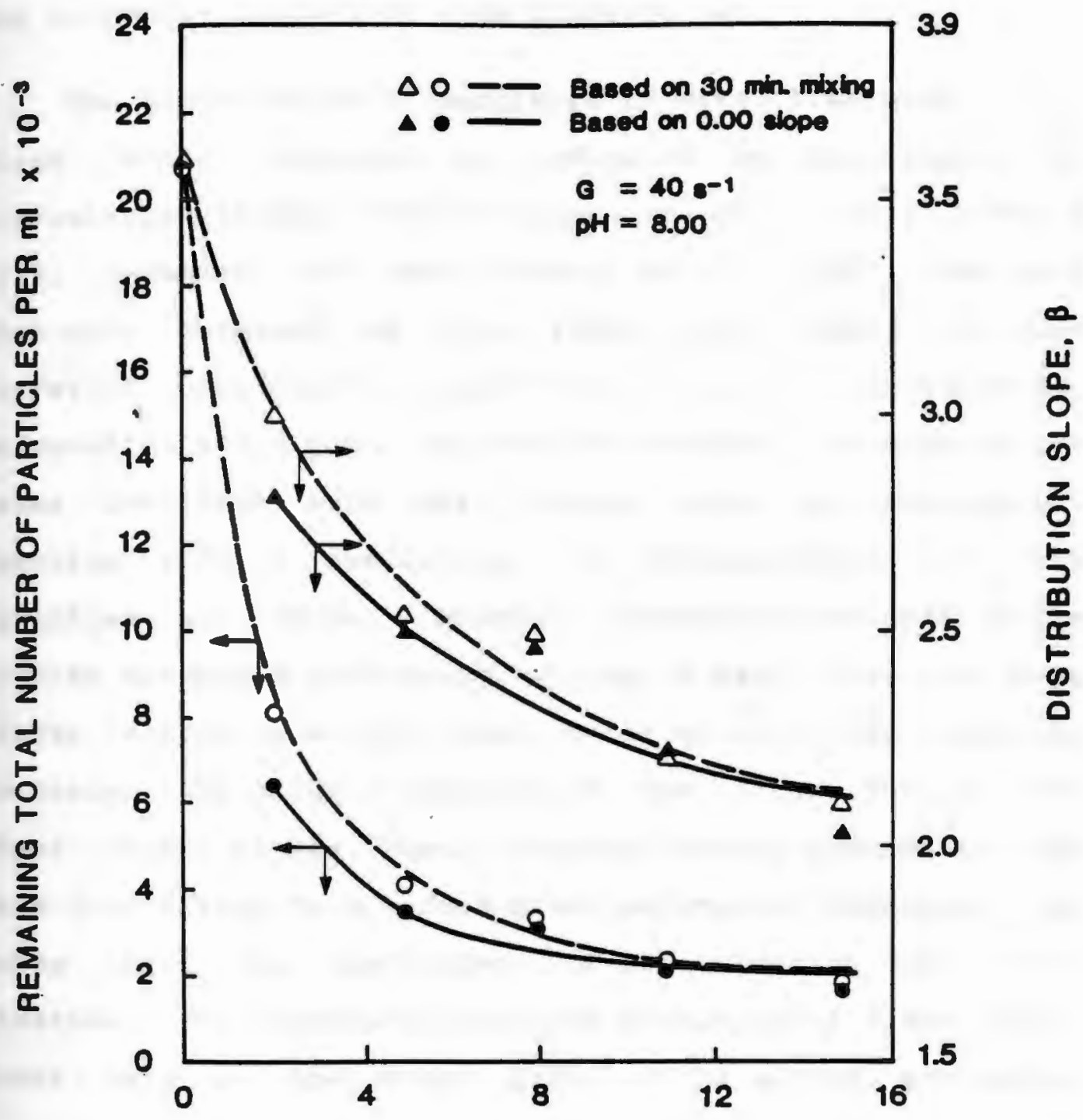

\section{ALUM DOSE ( $\mathrm{mg} / \mathrm{L}$ )}

Figure 4.18 Remaining total number of particles/ml and distribution slope versus coagulant dose at both 30 minutes mixing time and zero slope for jar test experiment number 6 . 
suspension had a very sharp optimal pH point at about 7.3 and the natural river water had an optimal pH of about 7.0 for turbidity removal by alum flocculation.

The flocculation of particles in water treatment is a first order reaction as indicated by the theory of flocculation (Audson 1965; Texippe et al. 1971; O'Melia 1972; Rawamura 1976; and Cornwell et al. 1983). The data that were obtained in this study were fitted to four different regression equations, linear, logarithmic, exponential and a power regression function. In most of the cases the data were best fitted with the exponential function with a coefficient of determination in the seventies and above. However, observing the data in the figures discussed previously, it can be seen that at some finite mixing time the total number of particles starts to increase, indicating a breakage in the flocs due to the shear forces on the flocs. Because of this phenomenon, the data were fitted to a second order polynomial function. By doing this, the coefficient of determination rose to the nineties. This indicates that the formation of flocs takes place only in the first part of the mixing, any mixing beyond this time will cause flocs to break resulting in a deviation from the theory of flocculation. The degree of breakage is a function of both the mixing intensity and the strength of the formed flocs. In a later discussion it will be shown that at a certain mixing intensity the total number of particles remaining will stay constant even at very long 
mixing times.

\subsubsection{Pilot Plant Mixing Tank Results}

Plots of the remaining total number of particles and the distribution slope as a function of mixing time for four different mixing intensities are shown in Figures 4.19, and 4.20, respectively. The data used for plotting are shown in Appendix B. The curves were drawn visually without using any kind of data fitting. From the two figures, 4.19 and 4.20 , it is clear that the rate of flocculation at the initial mixing times is proportional to the mixing intensity. Figure 4.21 is a plot of the rate of flocculation based on both the remaining particles and the distribution slope value for the initial 7 minutes of mixing. The best fit for the relationship between the rate of flocculation and the mixing intensity was found to be a linear relationship. The coefficients of determination were 0.88 and 0.78 for the relationships based on the total number of particles and the distribution slope value respectively. Similar findings were shown by Ives (1978b) and Lawler et al. (1983).

The time required to reach the maximum floc formation (optimum mixing time) is dependent on the mixing intensity. At the high mixing intensity ( $G=100 \mathrm{~s}^{-1}$ ) the optimum mixing time was about 15 minutes and 45 minutes was optimum for the low mixing intensity $(25 s-1)$. These values are based on the curves shown in Figures 4.19 and 4.20 . When the data of 


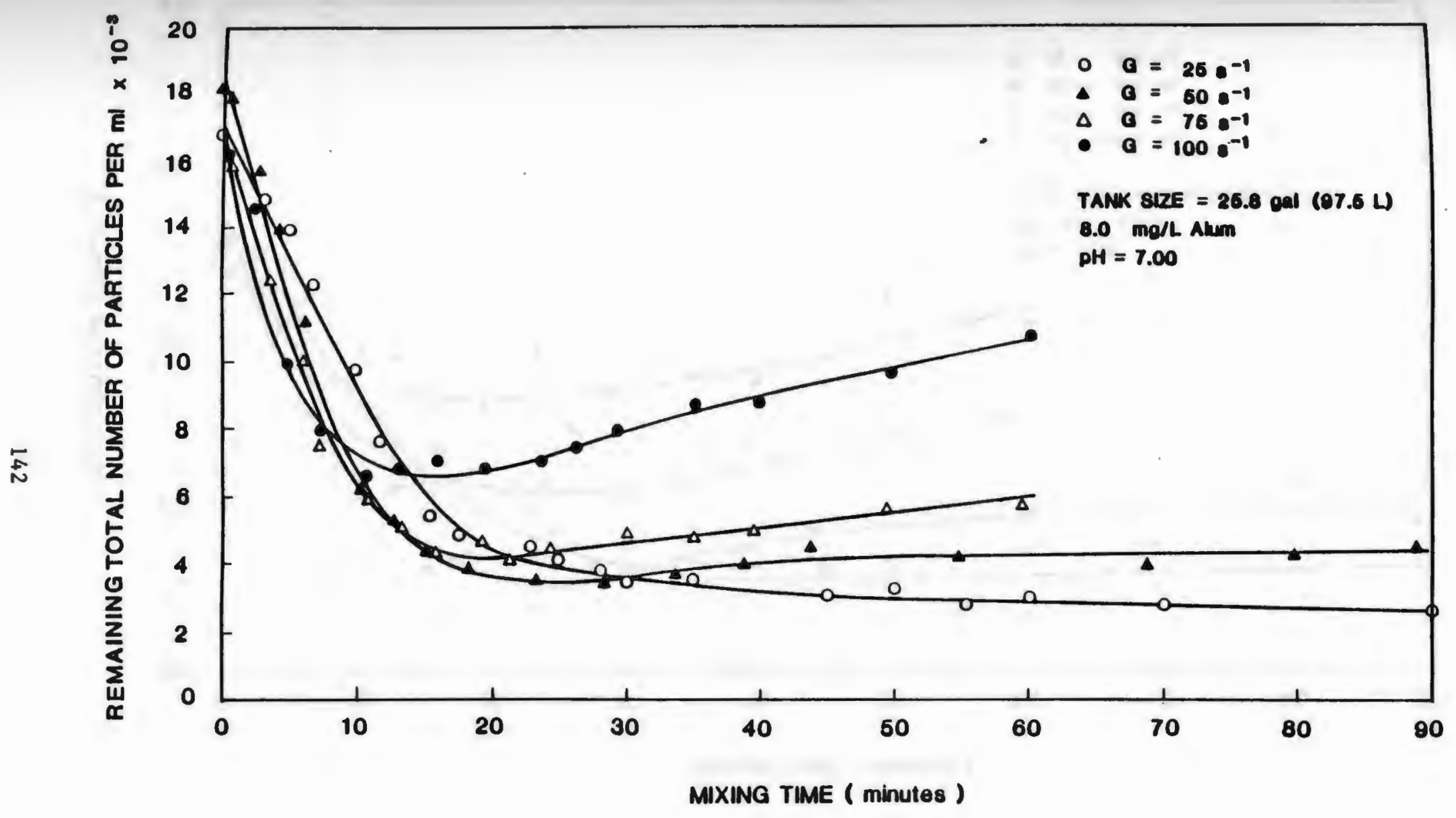

Figure 4.19 Remaining total number of particles/ml versus mixing time for four mixing intensities for the pilot plant mixing tank. 


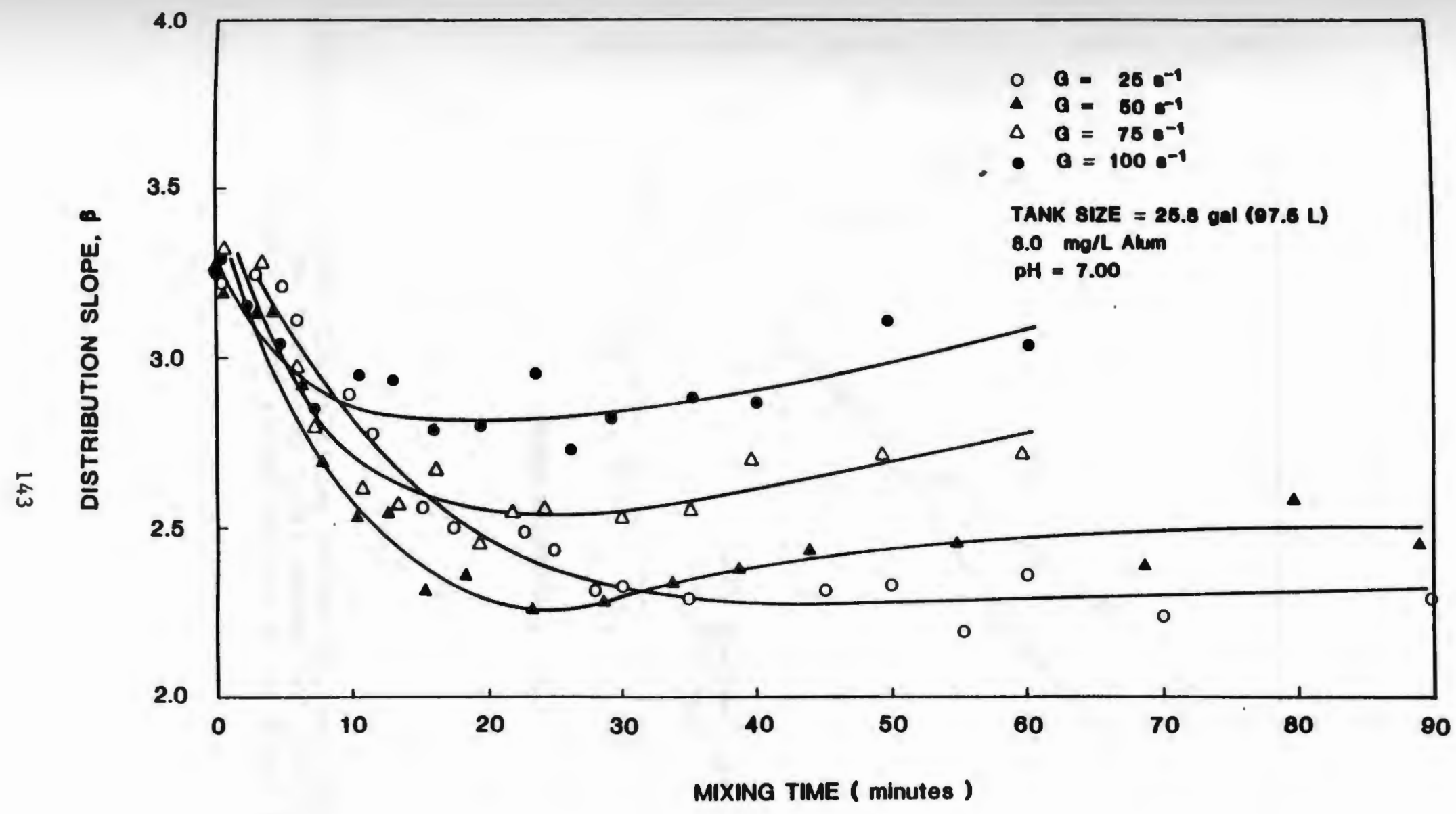

Figure 4.20 Distribution slope versus mixing time for four mixing intensities for the pilot plant mixing tank. 


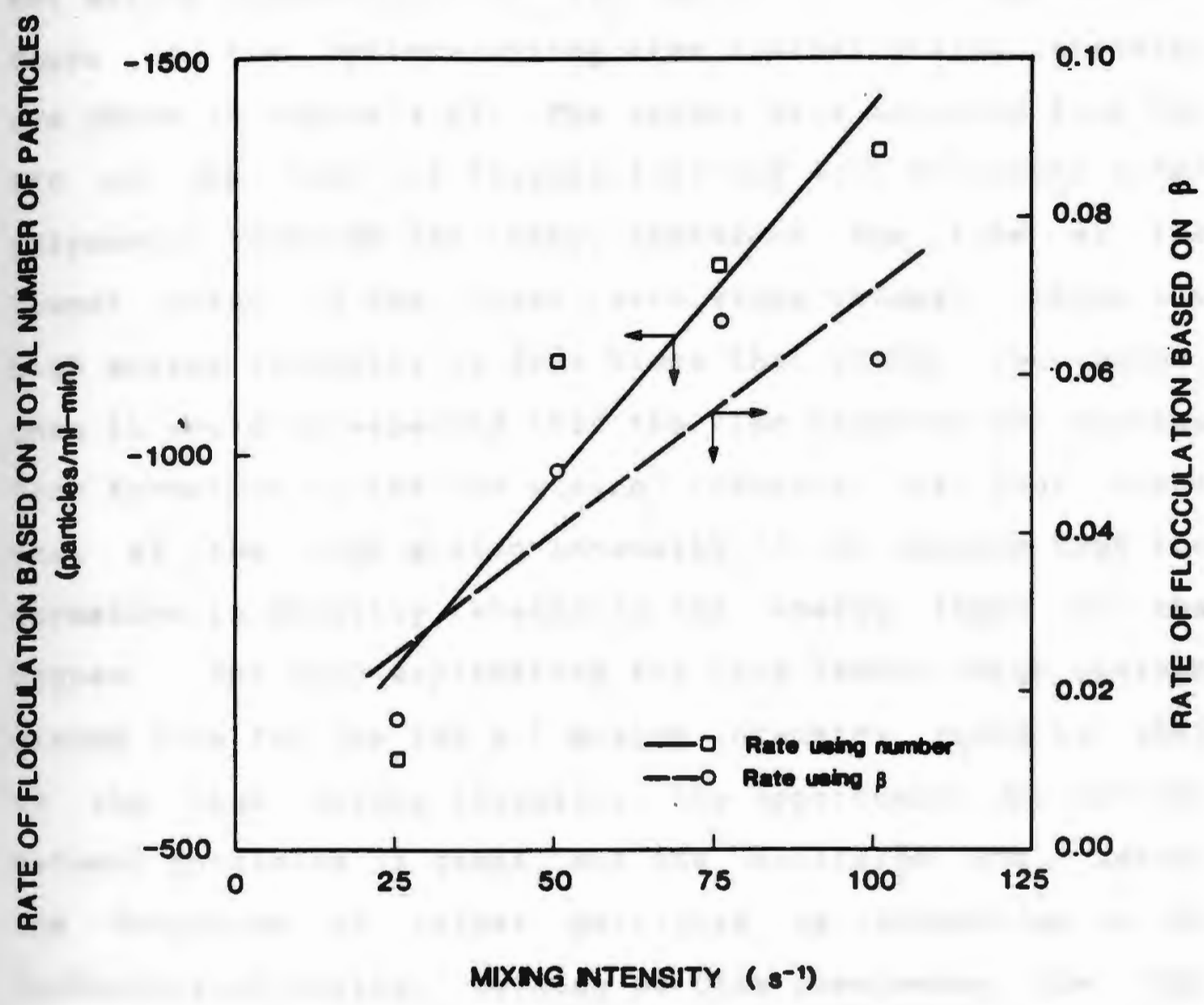

Figure 4.21 Rate of flocculation versus mixing intensity based on the remaining total number of particles and the distribution slope for the pilot plant mixing tank. 
Figures 4.19 and 4.20 were fitted to second order polynomial equation, the optimum mixing times were 30 and 45 minutes for mixing intensities of 100 and $25 \mathrm{~s}^{-1}$ respectively. plots of the optimum mixing time against mixing intensity are shown in igure 4.22 . The values were obtained from the fit of the data of Figures 4.19 and 4.20 to second order polynomial equation and they represent the time at the lowest point on the curves (zero slope values). Since the high mixing intensity is four times that of the low value, then it would be expected that the time required for maximum floc formation at the low mixing intensity is four times that at the high mixing intensity if one assumes that the formation is directly related to the energy input to the system. The only explanation for this result (high optimum mixing time for the $100 \mathrm{~s}^{-1}$ mixing intensity) could be that at the high mixing intensity, the opportunity for contact between particles is great, but the collision and, hence, the formation of larger particles is reduced due to the turbulence of mixing. Because of this phenomenon, the time needed to reach the maximum floc formation at the high mixing intensity was longer than what it should be. It is unlikely that the optimum mixing time for the low mixing intensity be 120 minutes.

After the optimum mixing time was reached, the curves start shifting upwards except at the lowest mixing intensity where it stays constant even at very high mixing time. The reason for this is the fact that flocs start to break and 


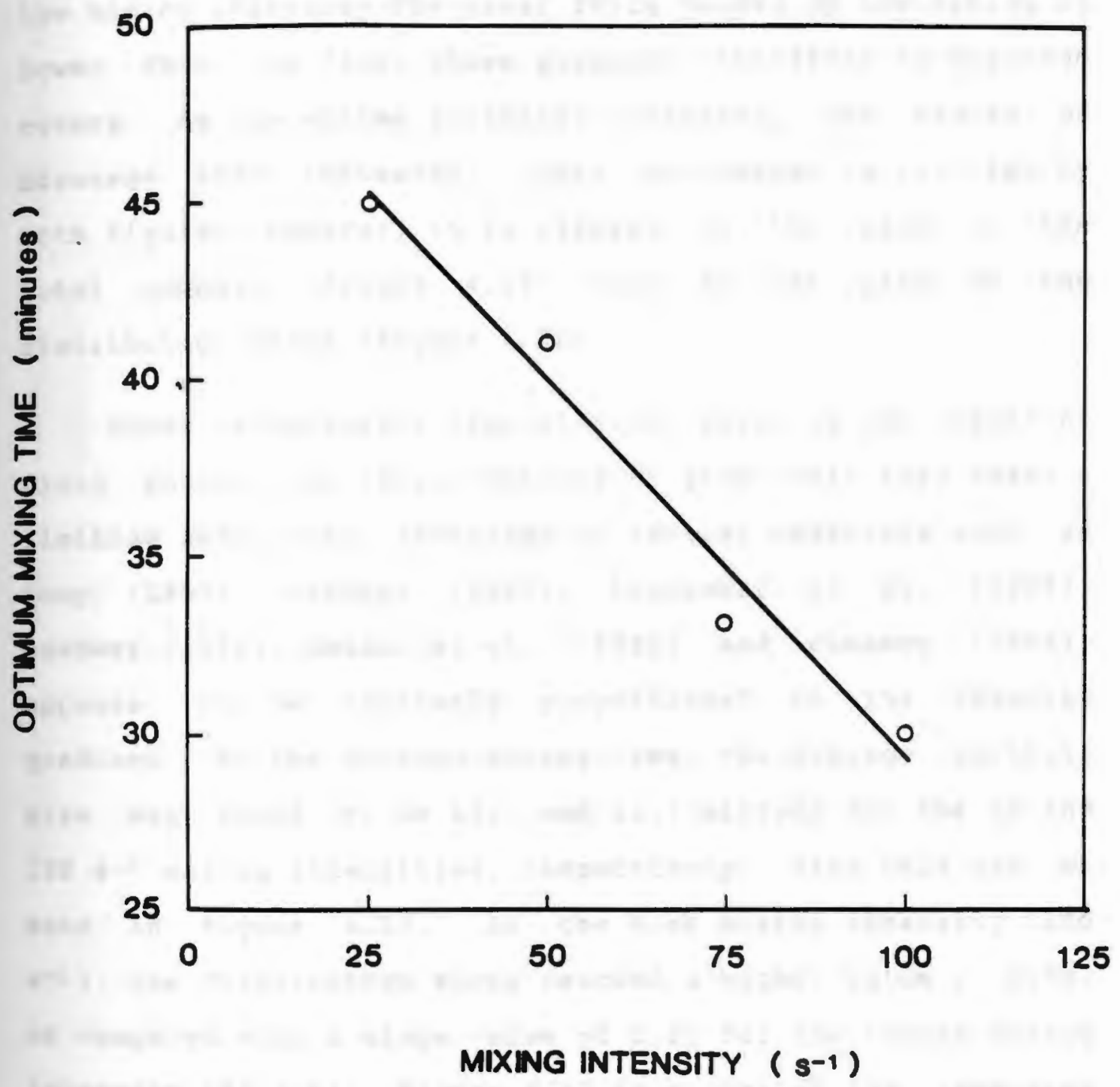

Figure 4.22 Optimum mixing time versus mixing intensity for the pilot plant mixing tank. 
the degree of breakage is a function of the mixing intensity, if all other variables are held constant. At a low mixing intensity the shear force caused by the mixing is lower than the flocs shear strength, therefore no breakage occurs. As the mixing intensity increases, the degree of breakage also increases. This phenomenon is verified by both figures, however, it is clearer in the plot of the total numbers (Figure 4.19) than in the plot of the distribution slope (Figure 4.20).

Under orthokinetic flocculation, which is the result of fluid motion, the flocs continue to grow until they reach a limiting size, that, according to several observers such as Camp (1955), Shinnar (1961); Lagvankar et al. (1968), Boadway (1978), Matsuo et al. (1981) and Cleasby (1984), appears to be inversely proportional to the velocity gradient. At the optimum mixing time, the average particle size was found to be 14.2 and 11.7 microns for the 25 and $100 \mathrm{~s}^{-1}$ mixing intensities, respectively. Also this can be seen in Figure 4.20. At the high mixing intensity $(100$ $s^{-1}$ ), the distribution slope reached a higher value, 2.80 , as compared with a slope value of 2.27 for the lowest mixing intensity $\left(25 \mathrm{~s}^{-1}\right)$. Figure 4.23 is a plot.of the remaining total number of particles and the distribution slope value against the mixing intensity. The points that were used in plotting the two curves represent the values at the optimum mixing time when the data of Figures 4.19 and 4.20 were fitted to a second order polynomial function. A high slope 


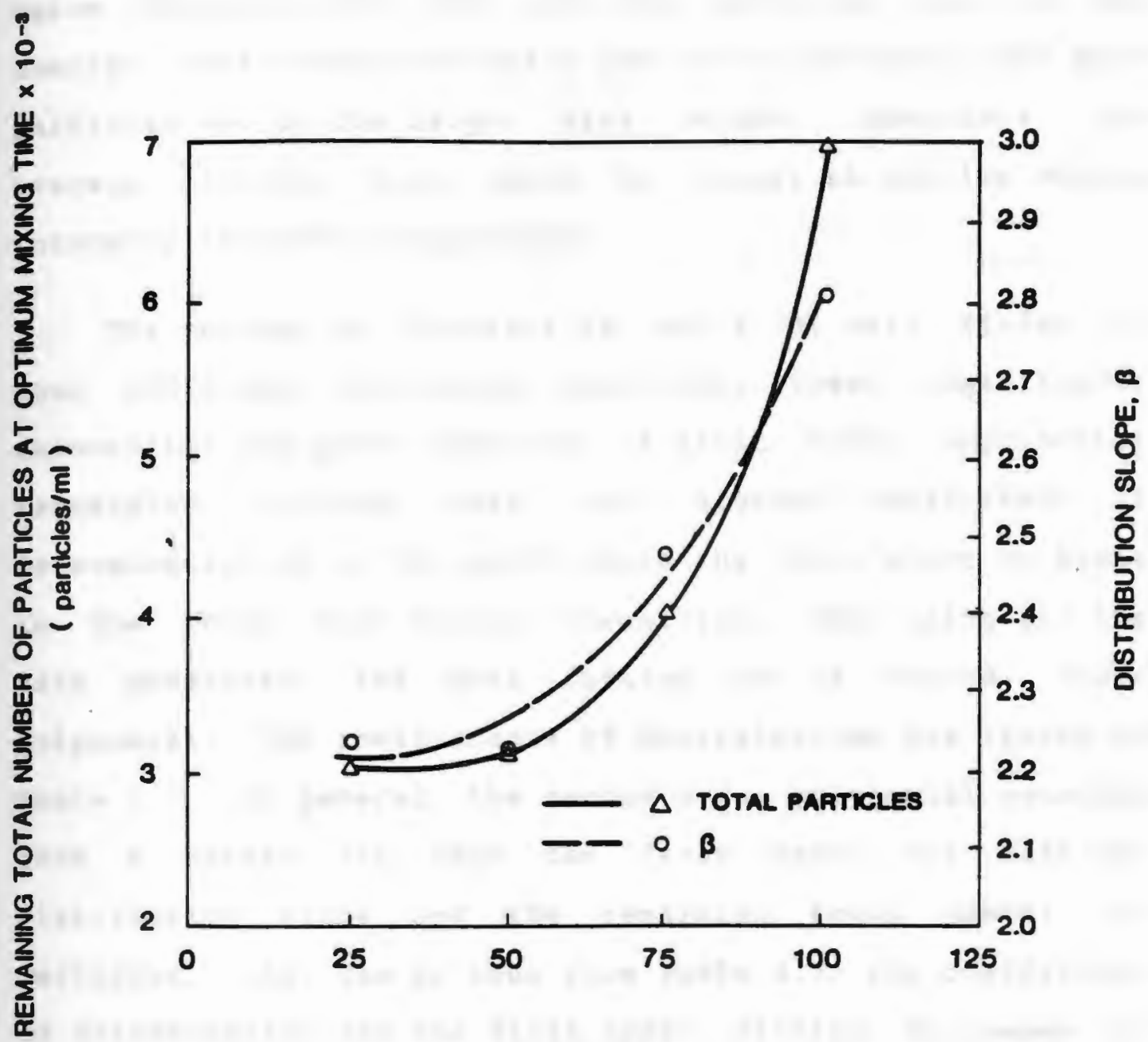

MIXING INTENSITY $\left(\boldsymbol{s}^{-1}\right)$

Figure 4.23 Remaining total number of particles/ml and distribution slope at optimum mixing time versus mixing intensity for the pilot plant mixing tank. 
value indicates that most of the particles are in the smaller size ranges whereas a low value indicates that more particles are in the larger size ranges. Therefore, the average particle size would be larger at the low mixing intensity in such a suspension.

The curves, in Figures 4.19 , and 4.20 , were fitted to four different regression equations, linear, logarithmic, exponential and power function. A first order exponential regression fitting gave the highest coefficient of determination up to the point where the flocs start to break in the three high mixing intensities. When using all the data generated, the best fitting was a second order polynomial. The coefficients of determination are listed in rable 4.7. In general, the second order polynomial equation gave a better fit than the first order for both the distribution slope and the remaining total number of particles. As can be seen from Table 4.7, the coefficient of determination for the first order fitting decreases in value as the mixing intensity increases for both the number and the slope. This indicates that the higher the mixing intensity, the more floc breakage. Figures $4.23 \mathrm{a}$ and $4.23 \mathrm{~b}$ were plotted in order to clear the point regarding the breakage of flocs after the optimum mixing time.

In both figures, the data from the 75 s-1 mixing intensity experiment were fitted to both a first and a second order polynomial equations for mixing times of 19 and 60 minutes, respectively. As can be seen from both figures 
Table 4.7- Coefficients of Determination Based on First and second Order. Polynomial Functions for Pllot plant Mixing rank

yixing

Intensity

$\left(s^{-1}\right)$
Coefficient of Determination

First Order

distribution

slope remaining distribution

particles second order slope remaining particles

\begin{tabular}{rllll}
25 & 0.75 & 0.79 & 0.96 & 0.98 \\
50 & 0.44 & 0.51 & 0.80 & 0.85 \\
75 & 0.27 & 0.35 & 0.78 & 0.84 \\
100 & 0.08 & 0.06 & 0.68 & 0.63 \\
\hline
\end{tabular}




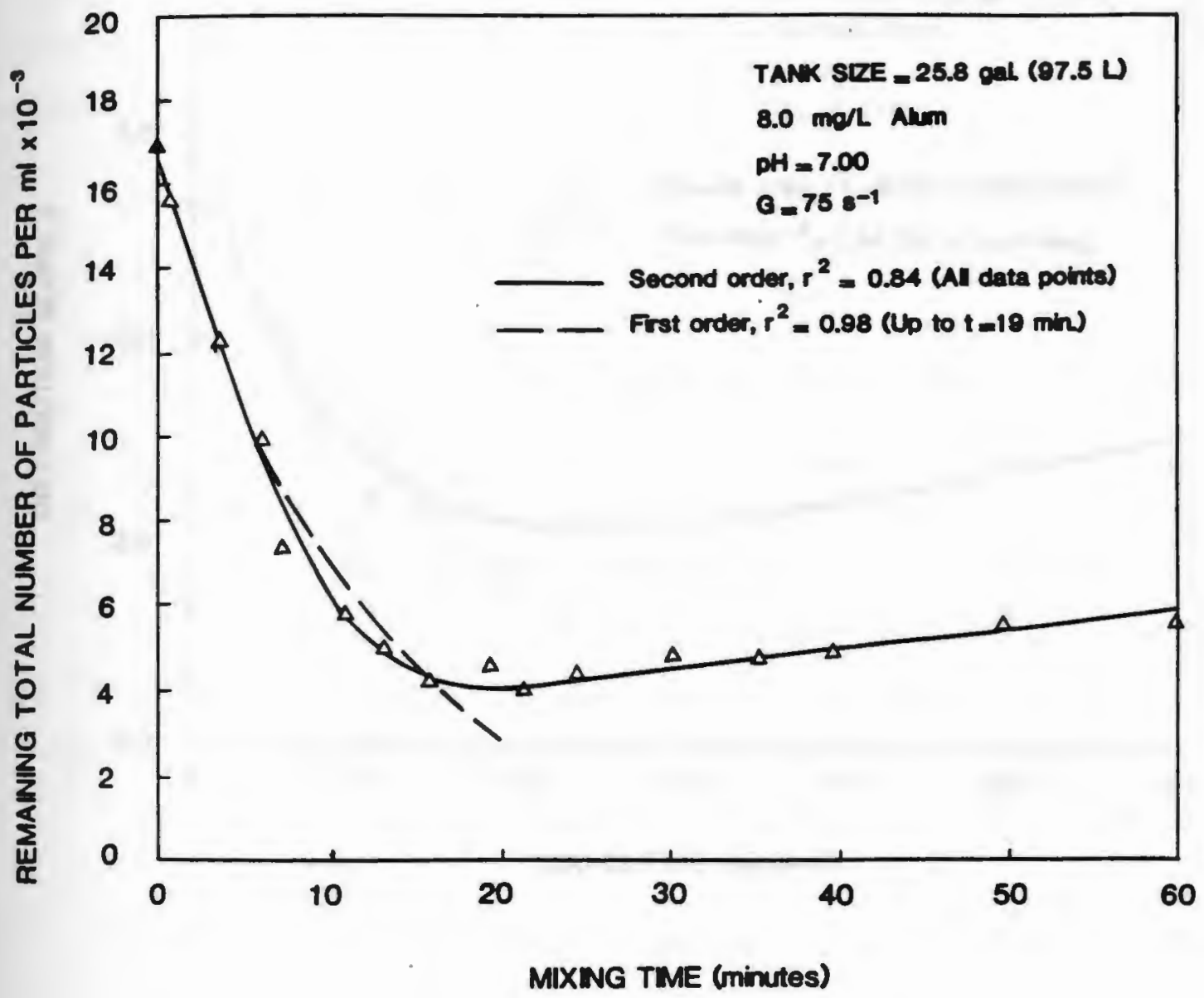

Figure 4.23a Comparison between first and second order polynomial equations for the pilot plant mixing tank using the remaining total number of particles data. 


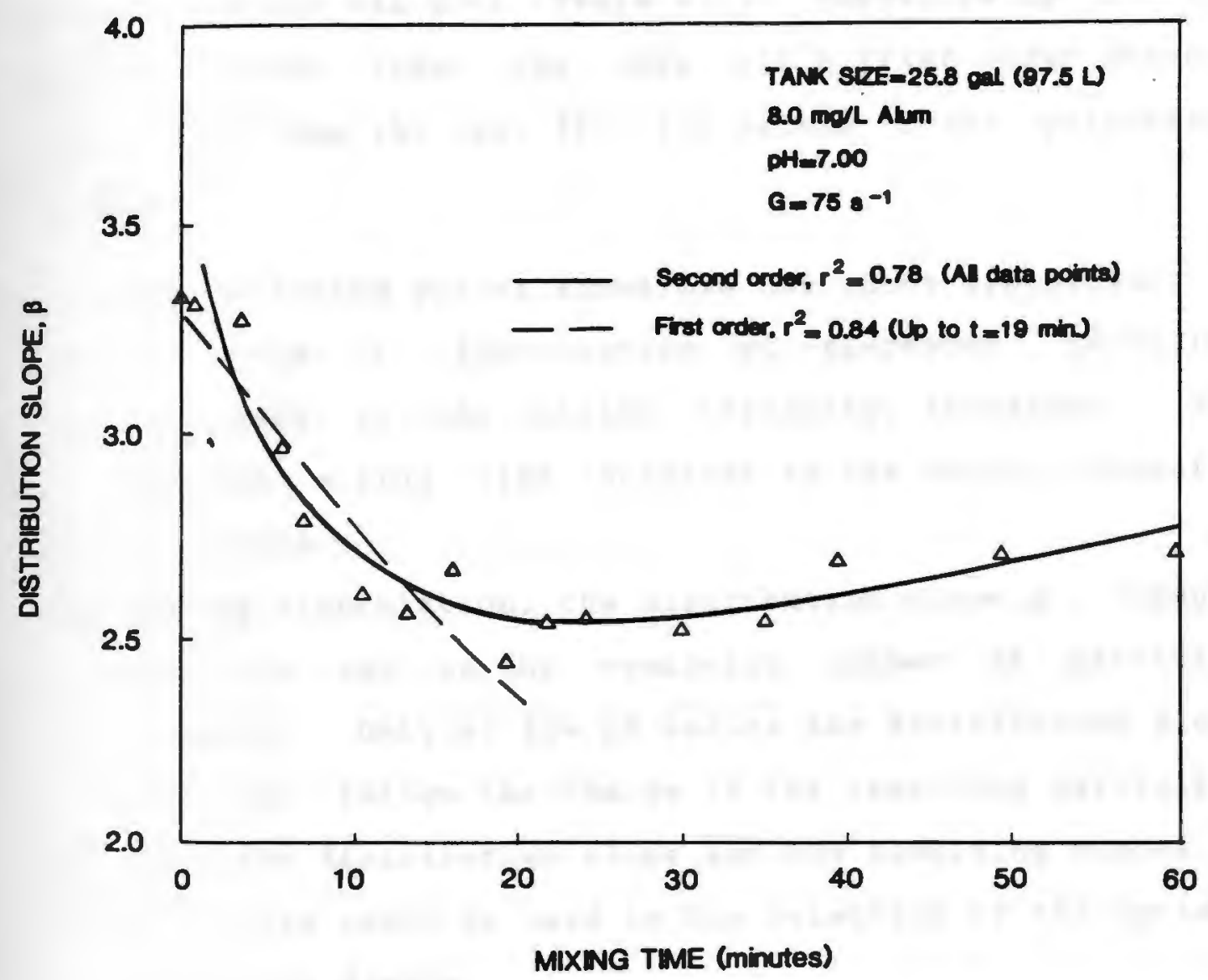

Figure 4.23b Comparison between first and second order polynomial equations for the pilot plant mixing tank using the distribution slope data. 
the first order polynomial functions gave a good fit up to a mixing time of 19 minutes but for a mixing time of 60 minutes the fit was poor (Table 4.7). Therefore up to the optimum mixing time, the data fit a first order whereas beyond that time the best fit is a second order polynomial function.

The following points summarize the above discussion:

(1) The rate of flocculation of suspended particles increases as the mixing intensity increases. The optimum mixing time increases as the mixing intensity decreases.

(2) During flocculation, the distribution slope, $\beta$, changes the same way as the remaining number of particles changes. Only at low pir values the distribution slope does not follow the change in the remaining particles.

(3) Both the distribution slope and the remaining number of particles could be used in the selection of the optimum coagulant dosage.

(4) The floc breakage is a function of the mixing intensity.

(5) Flocculation of suspended particles is a first order reaction only to a point where the primary flocs are formed. Above the optimum mixing time, the reaction was found to be second order due to floc breakage. 
4.2 Pilot plant Filtration

In the pilot plant filtration section, the results that were obtained from the 24 runs of the pilot plant will be presented and discussed. The section is divided into two subsections. The first subsection deals with the influence of the influent particle size distribution (PSD) on the effluent quality and PSD. The second subsection is a discussion of the influence of the influent PSD on the head loss which occurs during granular media filtration.

4.2.1 Influence of Influent Particle size distribution on Effluent Quality and Particle size Distribution

The three operational parameters that were used to change the particle size distribution of the raw water were the coagulant dosage, mixing intensity and time. The influence of the particle size distribution produced by each of these three parameters on the filtered water quality and PSD will be discussed. The data generated from all of the 24 runs are 1 isted in Appendix $C$.

The data that were obtained from the pilot plant were used to calculate the filter coefficient, specific deposit, and the Filterability Number. The parameters are required in order to be able to compare the effect of the different influent suspensions on the effluent quality. However, due to the fact that the ratio of the influent to the effluent particle count is very high, those parameters did not prove 
to be useful tools for comparison purposes. As an example, the influent particle count was about 16,000 particles/ml and with fine media $(0.35 \mathrm{~mm})$, the effluent particle count was less than 10 particles/ml in most of the runs independent of influent conditions. Therefore, no obvious trend in the data were observed with respect to the above three parameters. Due to these problems, qualitative comparisons and observations will be presented.

\subsubsection{Influence of Coagulant Dosage}

Figure 4.24 is a plot of the effluent total number of particles/ml against the run time for the fine sand media $(0.35 \mathrm{~mm})$. Each curve represents a different coagulant dosage. When no alum was added to the influent, the effluent total particle count started at a high value then it improved rapidly reaching its lowest value at time 3 hours from the start. No constant effluent concentration was observed in this condition. The filtration efficiency remained constant for a short period and then rapidly deteriorated. With the addition of $2.0 \mathrm{mg} / \mathrm{L}$ of alum the removal of particles improved and the rate of deterioration was not as rapid. The rate of deterioration is defined as the total number of particles that pass through the sand bed per milliliter per one hour of run time. The rate of deterioration is calculated for the region after which the effluent particle count starts rising. The rate of deterioration was 29.3 and 0.30 particles/ml-hour for the 


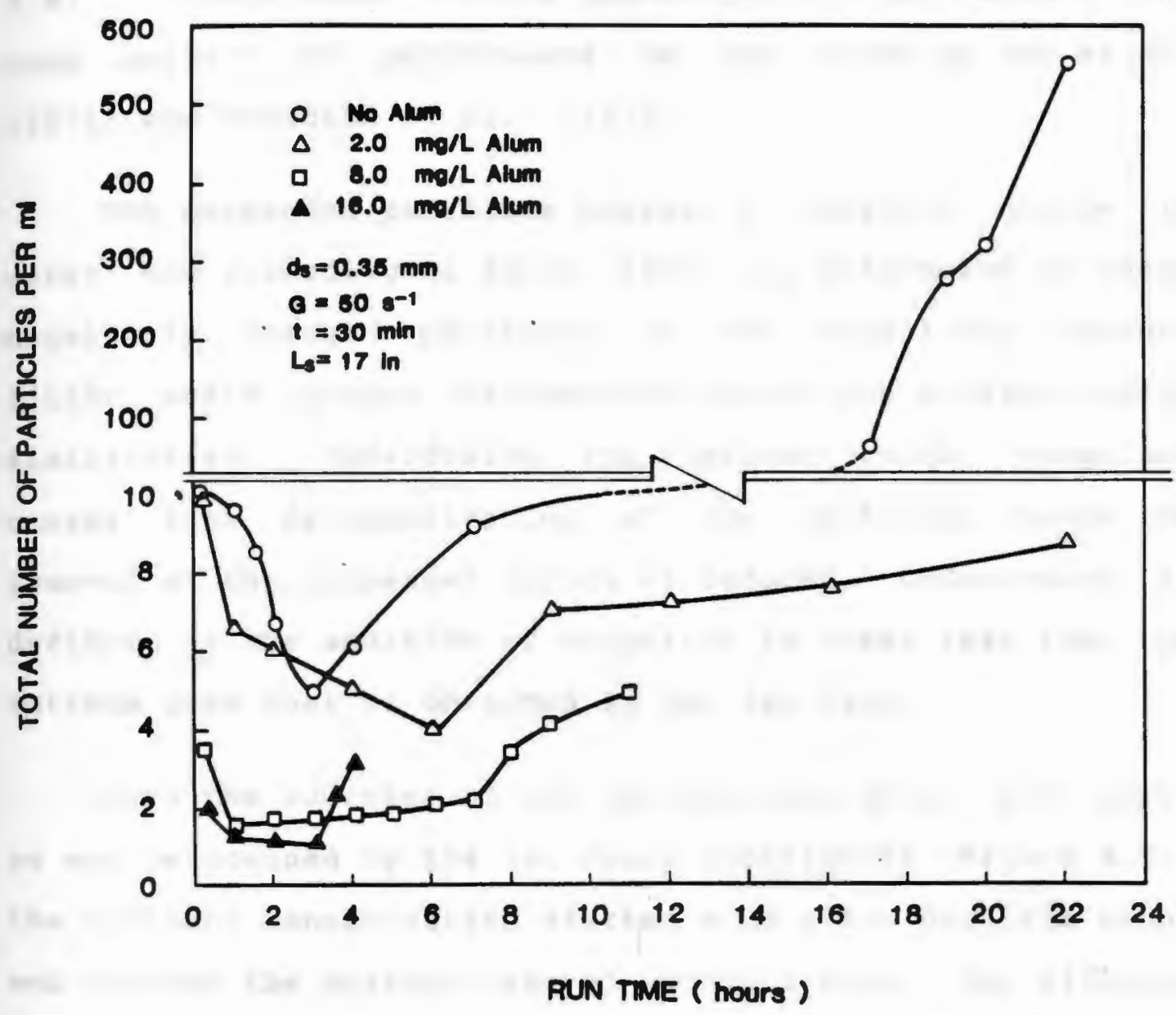

Figure 4.24 Effluent total number of particles/ml versus run time for fine sand with various alum dosages. 
0.0 and $2.0 \mathrm{mg} / \mathrm{L}$ influent alum dosages, respectively (Table 4.8). Filters which receive underdosed influent showed the same pattern of performance as was shown by yao et al. (1971) and Habibian et al. (1975).

The suspended particles possess a negative charge in water and according to Smith (1967) the attachment of these negatively charged particles to the negatively charged filter media grains is impaired due to the surface charge similarities. Underdosing the influent with coagulant causes less destabilization of the particles hence the removal of the suspended solids is reduced. Underdosing is defined as the addition of coagulant in doses less than the optimum dose that is obtained in the jar test.

With the addition of the optimum alum dose, $8.0 \mathrm{mg} / \mathrm{L}$, as was determined by the jar tests experiments (Figure 4.3), the effluent concentration started with a low particle count and reached the maximum removal within 1 hour. The effluent quality stayed constant for a longer period (about 7 hours) then deteriorated at a rate higher than when using $2.0 \mathrm{mg} / \mathrm{L}$ coagulant dosage. The rate of deterioration was 0.70 particles/ml-hour (Table 4.8). This rapid deterioration was not expected to occur. This is because the use of optimum dosage along with fine media would result in the termination of the run before any breakthrough would occur. 
Table 4.8- Rates of Effluent Quality Deterioration for Filter No. 1

\begin{tabular}{|c|c|c|c|c|c|}
\hline $\begin{array}{l}\text { Influent } \\
\text { Type }\end{array}$ & $\begin{array}{l}\text { Dete } \\
\text { start } \\
(\text { hr) }\end{array}$ & $\begin{array}{r}\text { oration } \\
\text { End } \\
(\mathrm{hr})\end{array}$ & $\begin{array}{c}\text { Total Particle } \\
\text { start }\end{array}$ & $\begin{array}{l}\text { Count at } \\
\text { End }\end{array}$ & $\begin{array}{l}\text { Rate of } \\
\text { Deterioration } \\
\text { (number } / \mathrm{ml}-\mathrm{hr} \text { ) }\end{array}$ \\
\hline 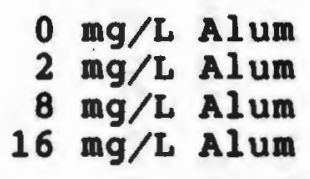 & $\begin{array}{l}3 \\
6 \\
7 \\
3\end{array}$ & $\begin{array}{r}22 \\
22 \\
11 \\
4\end{array}$ & $\begin{array}{l}4.9 \\
3.9 \\
2.3 \\
1.1\end{array}$ & $\begin{array}{r}558.3 \\
8.9 \\
4.9 \\
3.2\end{array}$ & $\begin{array}{r}29.1 \\
0.3 \\
0.7 \\
2.0\end{array}$ \\
\hline $\begin{array}{l}G=25 s^{-1} \\
G=50 \\
s^{-1} \\
G=100 s^{-1}\end{array}$ & $\begin{array}{l}6 \\
7 \\
6\end{array}$ & $\begin{array}{l}9 \\
11 \\
12.75\end{array}$ & $\begin{array}{l}0.9 \\
2.3 \\
2.3\end{array}$ & $\begin{array}{l}2.6 \\
4.9 \\
4.7\end{array}$ & $\begin{array}{l}0.6 \\
0.7 \\
0.4\end{array}$ \\
\hline $\begin{array}{l}t=15 \mathrm{~min} \\
t=30 \mathrm{~min} \\
t=45 \mathrm{~min}\end{array}$ & $\begin{array}{l}4 \\
7 \\
3\end{array}$ & $\begin{array}{l}4.75 \\
11 \\
12\end{array}$ & $\begin{array}{l}4.6 \\
2.3 \\
1.5\end{array}$ & $\begin{array}{l}6.0 \\
4.9 \\
4.3\end{array}$ & $\begin{array}{l}1.8 \\
0.7 \\
0.3\end{array}$ \\
\hline
\end{tabular}


overdosing $(16.0 \mathrm{mg} / \mathrm{L})$ the influent with coagulant caused a slight decrease in the initial particle count and a short region of constant particle count ( 2 hours). The effluent deteriorated very rapidly ( 2.0 particles/ml-hour) after 3 hours from the start. At this alum concentration $(16.0 \mathrm{mg} / \mathrm{L})$, the flocs that were formed were large in size (visual observations) and the mass of solids was high compared to the other coagulant dosages, 0,2 , and $8 \mathrm{mg} / \mathrm{L}$. This caused rapid head loss development, therefore a shorter filter run. It is expected that the effluent quality will remain constant at the end of the run. This is because solid loading was high which resulted in rapid head loss development therefore the run was terminated before any particles start to pass through the sand bed. The possible cause of effluent deterioration could be the detachment of particles that were previously retained within the bed.

The runs were terminated because the head available was exhausted. In all the runs for filter number $1(0.35 \mathrm{~mm})$ the effluent never reached $10 \%$ of the influent total particle count even for the run with no alum. The $10 \%$ influent corresponds to about $0.35 \mathrm{NTU}$ which is below the U.S. EPA standards. Influent as well as effluent turbidities were measured from time to time as quick indicator of the quality of either suspensions. 
Using the fine sand media, almost all of the influent particles were removed even when no alum was added to the influent. The removal was largely due to straining and sedimentation in the top layer of the filter. The addition of coagulant improved the effluent quality but the filter run length was shortened. The improvement in the effluent quality was not significant with the increase in the coagulant dosage. The effluent quality was very good even at the $2.0 \mathrm{mg} / \mathrm{L}$ dose. This indicates that in direct filtration, the majority of particles can be removed by using fine media sand and low coagulant dosages (below the optimum). There appears to be no need to add coagulant in concentrations equal to the optimum or greater as determined by the jar test experiments $(8.0 \mathrm{mg} / \mathrm{L})$. High coagulant dosages will result in a short filter run and only a slight improvement in the effluent quality.

Based on these results, $8.0 \mathrm{mg} / \mathrm{L}$ alum provides the longest run time, the longest constant effluent region and gives the minimum effluent particle count based on the total volume produced compared with the other dosages investigated.

As the sand grain diameter increases, the effluent quality deteriorates. This is shown in Figure 4.25 for filter number $2(0.50 \mathrm{~mm})$. Only when no alum was added did the effluent reach $10 \%$ of the influent total particle count. The other alum dosages caused the filter to be clogged before $10 \%$ was reached. Therefore, the run termination was 


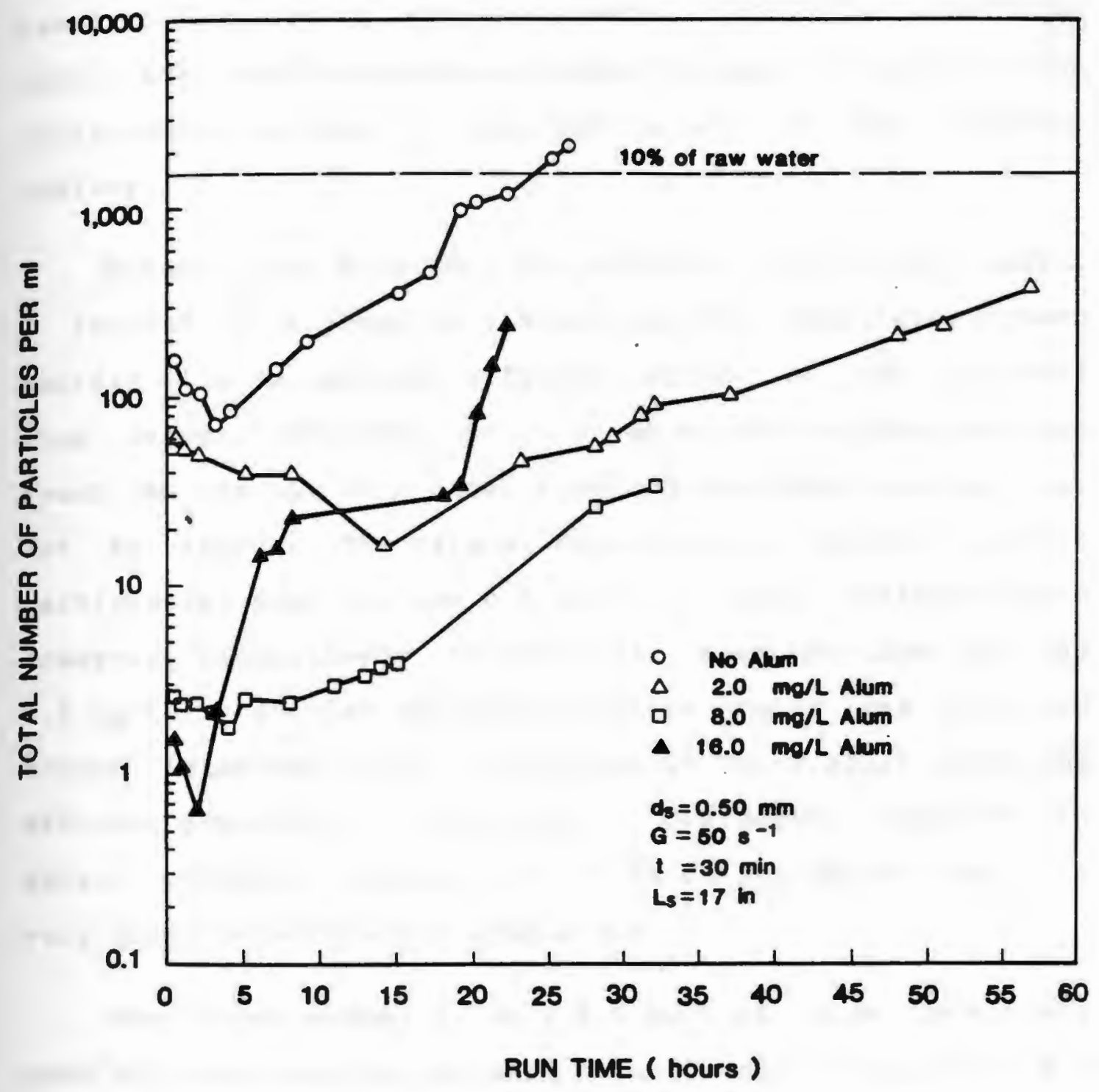

Figure 4.25 Effluent total number of particles/ml versus run time for medium sand with various alum dosages. 
based on the loss of available head $(7.5 \mathrm{ft})$. As with finer sand, the same observations could be made in regard to the relationship between the coagulant dosage and the effluent quality.

without alum addition, the effluent from filter number 2 reached a minimum in 3 hours and then deteriorated very rapidly with no constant effluent region. As the influent alum dosage increased to $2.0 \mathrm{mg} / \mathrm{L}$, the minimum particle count was reached at a later time and the deterioration was not as rapid. The rate of deterioration was 59.9 and 8.8 particles/ml-hour for the 0.0 and $2.0 \mathrm{mg} / \mathrm{L}$ influent alum dosages, respectively (Table 4.9). When the alum dose was $8.0 \mathrm{mg} / \mathrm{L}$ the initial effluent particle count was low and stayed relatively low for about 15 hours after which the effluent started to deteriorate. Overdosing resulted in better effluent quality for a short period of time but a very sharp deterioration (Table 4.9).

For filter number 2 , only $8.0 \mathrm{mg} / \mathrm{L}$ of alum gave very good effluent quality for more than 20 hours. Below the 8.0 $\mathrm{mg} / \mathrm{L}$ dose, the effluent quality was poor and above that dose the rate of deterioration was very high. Therefore, only the optimum dosage seem to give the best results with medium size media.

Using coarse media (filter number 3 , sand size $1.00 \mathrm{~mm}$ ) the effluent quality was not as good as that produced by fine and medium sand. The minimum particle count was about 
Table 4.9- Rates of Effluent Quality Deterioration for Filter No. 2

\begin{tabular}{|c|c|c|c|c|c|c|}
\hline $\begin{array}{l}\text { Influent } \\
\text { Type }\end{array}$ & $\begin{array}{l}\text { Det } \\
\text { start } \\
\text { (hr) }\end{array}$ & $\begin{array}{l}\text { tion } \\
\text { End } \\
(\mathrm{hr})\end{array}$ & $\begin{array}{r}\text { Total Pa } \\
\text { start }\end{array}$ & t1c1e & $\begin{array}{l}\text { Count at } \\
\text { End }\end{array}$ & $\begin{array}{c}\text { Rate of } \\
\text { Deterioration } \\
\text { (number } / m \text { m-hr) }\end{array}$ \\
\hline $\begin{array}{rll}0 & \mathrm{mg} / L & \text { Al um } \\
2 & \mathrm{mg} / L & \text { Al um } \\
8 & \mathrm{mg} / L & \text { Al um } \\
16 & \mathrm{mg} / \mathrm{L} & \text { Al um }\end{array}$ & $\begin{array}{r}3 \\
14 \\
8 \\
2\end{array}$ & $\begin{array}{l}22 \\
57 \\
32 \\
22\end{array}$ & $\begin{array}{r}73 \\
16 \\
2 \\
0.6\end{array}$ & & $\begin{array}{r}1211 \\
396 \\
34 \\
246\end{array}$ & $\begin{array}{r}59.9 \\
8.8 \\
1.3 \\
12.3\end{array}$ \\
\hline $\begin{array}{l}G=25 s^{-1} \\
G=50 \\
s^{-1} \\
G=100 s^{-1}\end{array}$ & $\begin{array}{r}20 \\
8 \\
22\end{array}$ & $\begin{array}{l}35.25 \\
32 \\
38\end{array}$ & $\begin{array}{l}0.9 \\
2 \\
2\end{array}$ & & $\begin{array}{r}92 \\
34 \\
731\end{array}$ & $\begin{array}{r}6 \\
1 \\
46\end{array}$ \\
\hline $\begin{array}{l}t=15 \mathrm{~min} \\
t=30 \mathrm{~min} \\
t=45 \mathrm{~min}\end{array}$ & $\begin{array}{l}7 \\
8 \\
6\end{array}$ & $\begin{array}{l}14 \\
32 \\
36\end{array}$ & 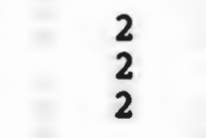 & & $\begin{array}{r}17 \\
34 \\
317\end{array}$ & $\begin{array}{r}2 \\
1 \\
11\end{array}$ \\
\hline
\end{tabular}


150 particles/ml when the coagulant dosage was $16.0 \mathrm{mg} / \mathrm{L}$ (Figure 4.26). The effluent particle count improved with the increase in the coagulant dosage but still remained very high compared to average public water supplies. At high alum dosage it was expected that the effluent quality would improve and stay constant for a longer period of time before starting to deteriorate. This is because at this coagulant dosage $(16.0 \mathrm{mg} / \mathrm{L})$, the solids loading to the filter is high and the flocs are large in size. Therefore most of the formed flocs would be removed by straining on top of the media. These deposited flocs would then act as a fine media in which the effluent quality would improve later in the run. But looking at Figure 4.26 and Table 4.10, the effluent deteriorated very rapidly after just 6 hours from the start of the run. It is possible that at this high dosage particles are detached very easily from the sand grains causing the rapid increase in the effluent particle count. Another explanation is that the media grains are completely covered with the large flocs and the particle to particle attachment was not enough to cause the removal of particles. Some researchers believe that high coagulant dosages produce weak flocs that do not easily attach to sand media or even to their own. Nevertheless, the same patterns for the effluent quality profile occurred as with the fine and medium sand. However, there is no major difference in the effluent quality between the 8.0 and $16.0 \mathrm{mg} / \mathrm{L}$ alum dosages at the early hours of operation (the total particle count at 3 hours of run time was 183 and 142 particles/mI 


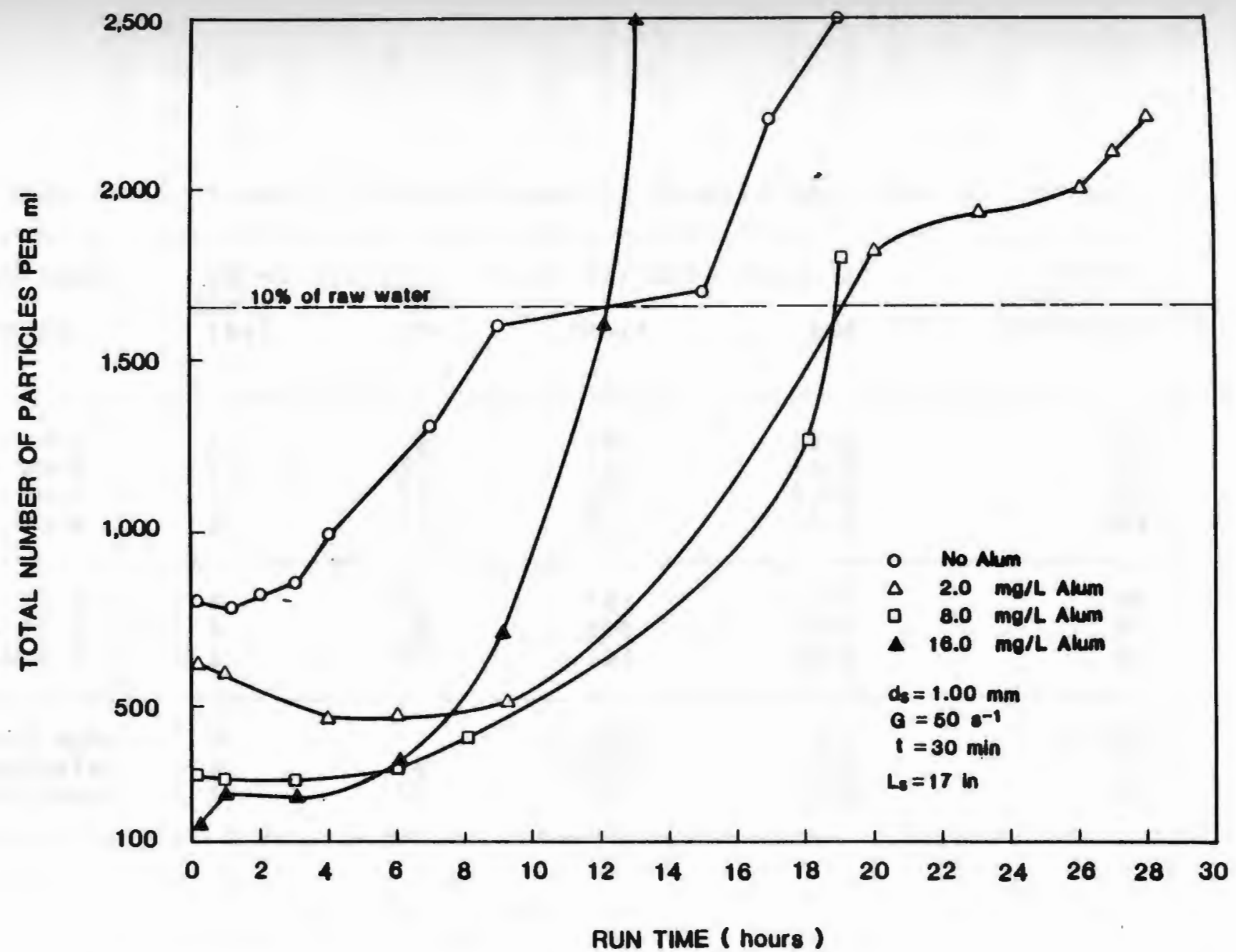

Figure 4.26 Effluent total number of particles/ml versus run time for coarse sand with various alum dosages. 
Table 4.10- Rates of Effluent Quality Deterioration for Filter No. 3

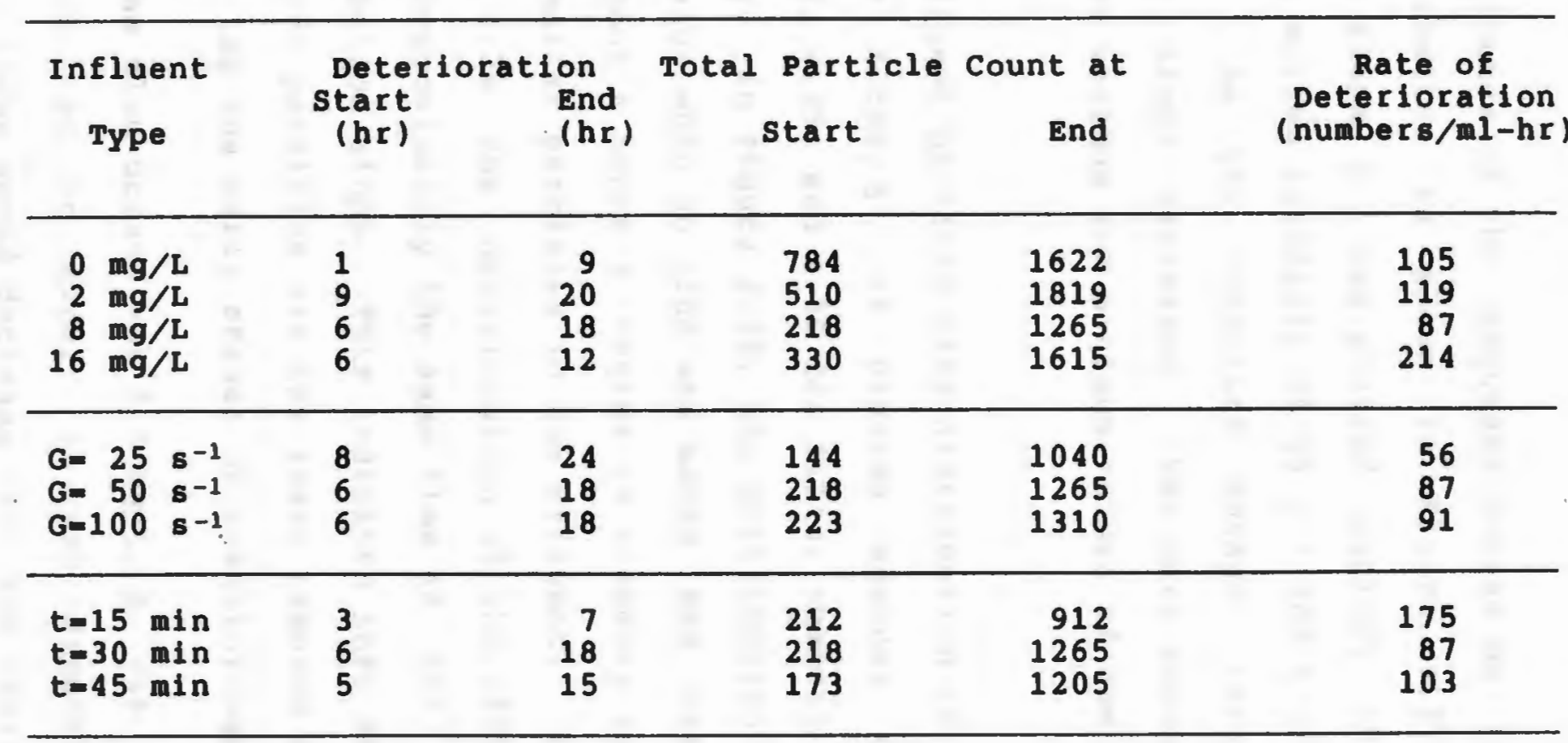


for 8.0 and $16.0 \mathrm{mg} / \mathrm{L}$ of influent coagulant dosages, respectively).

The influence of the coagulant dosage on the particle size distribution is shown in Figure 4.27 where the distribution slope, $\beta$, was plotted against the coagulant dosage for a mixing intensity of $50 \mathrm{~s}^{-1}$ and a mixing time of 30 minutes. As the coagulant dosage increased, the distribution slope decreased. The bars around each point represent the maximum and minimum values of the distribution slope.

The effluent particle size distribution in terms of the distribution slope, $\beta$, is plotted against run time in Figures $4.28,4.29$, and 4.30 for filter numbers 1,2 , and 3 , respectively. In Figure 4.28 , the distribution slope starts at a high value when no alum was added and decreases with time. At about 4 hours $\beta$ begins to steadily increase which indicates smaller particles in the effluent. Looking back at Figure 4.24 the deterioration of the effluent quality started at approximately the same time as the increase in the distribution slope. This indicates that with fine sand media, smaller particles are the least removed and the most dominant during the early stages of breakthrough.

When the alum dosage was $2.0 \mathrm{mg} / \mathrm{L}, \beta$ was unexpectedly higher than at no alum. It was expected that the distribution slope would decrease with the increase in the alum dosage as with the 8.0 and $16.0 \mathrm{mg} / \mathrm{L}$ dosages. One 


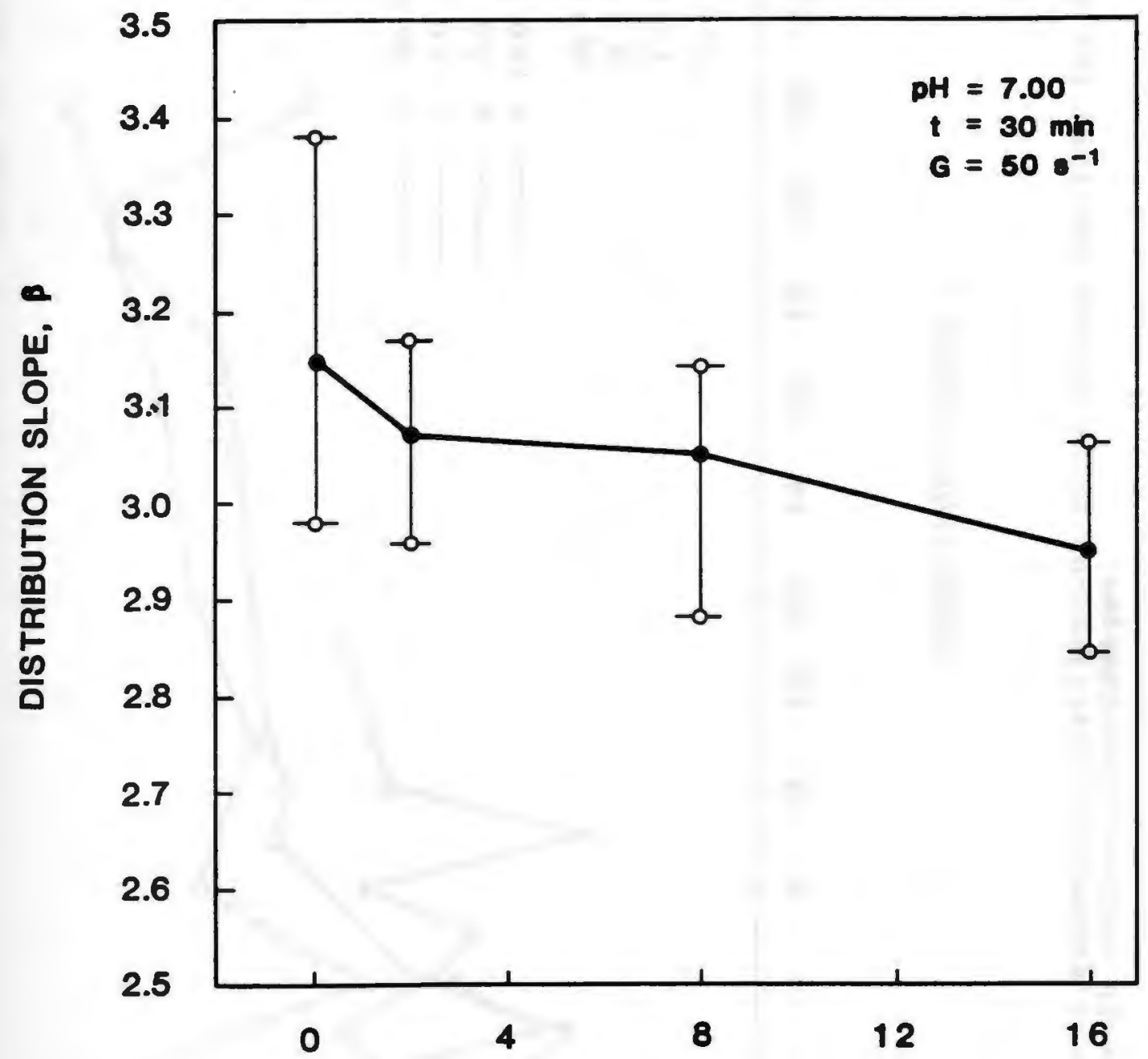

ALUM DOSE ( $\mathrm{mg} / \mathrm{L}$ )

Figure 4.27 Influent suspension distribution slope versus coagulant dose for the pilot plant experiments. 


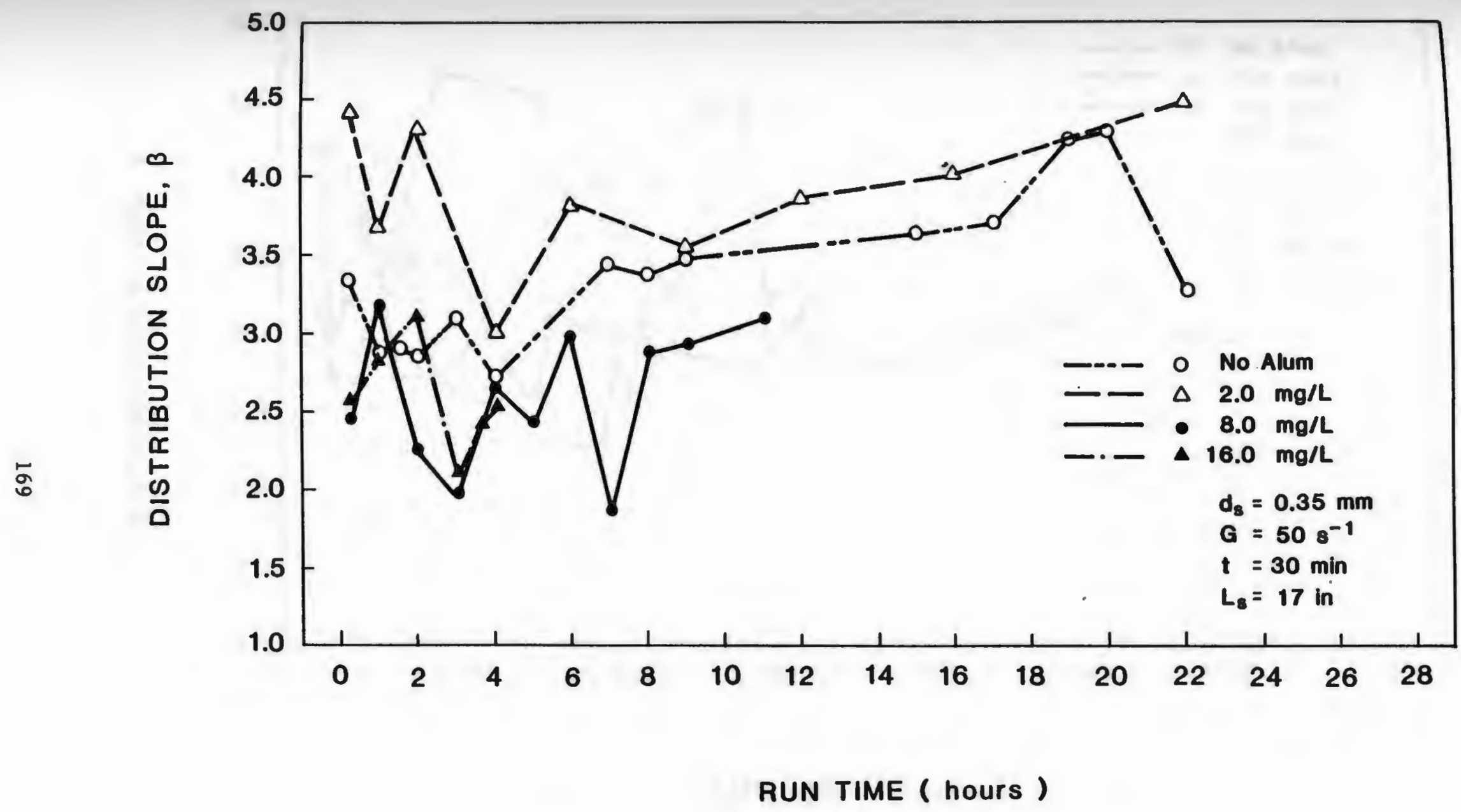

Figure 4.28 Effluent suspension distribution slope versus run time for fine sand with various alum dosages. 


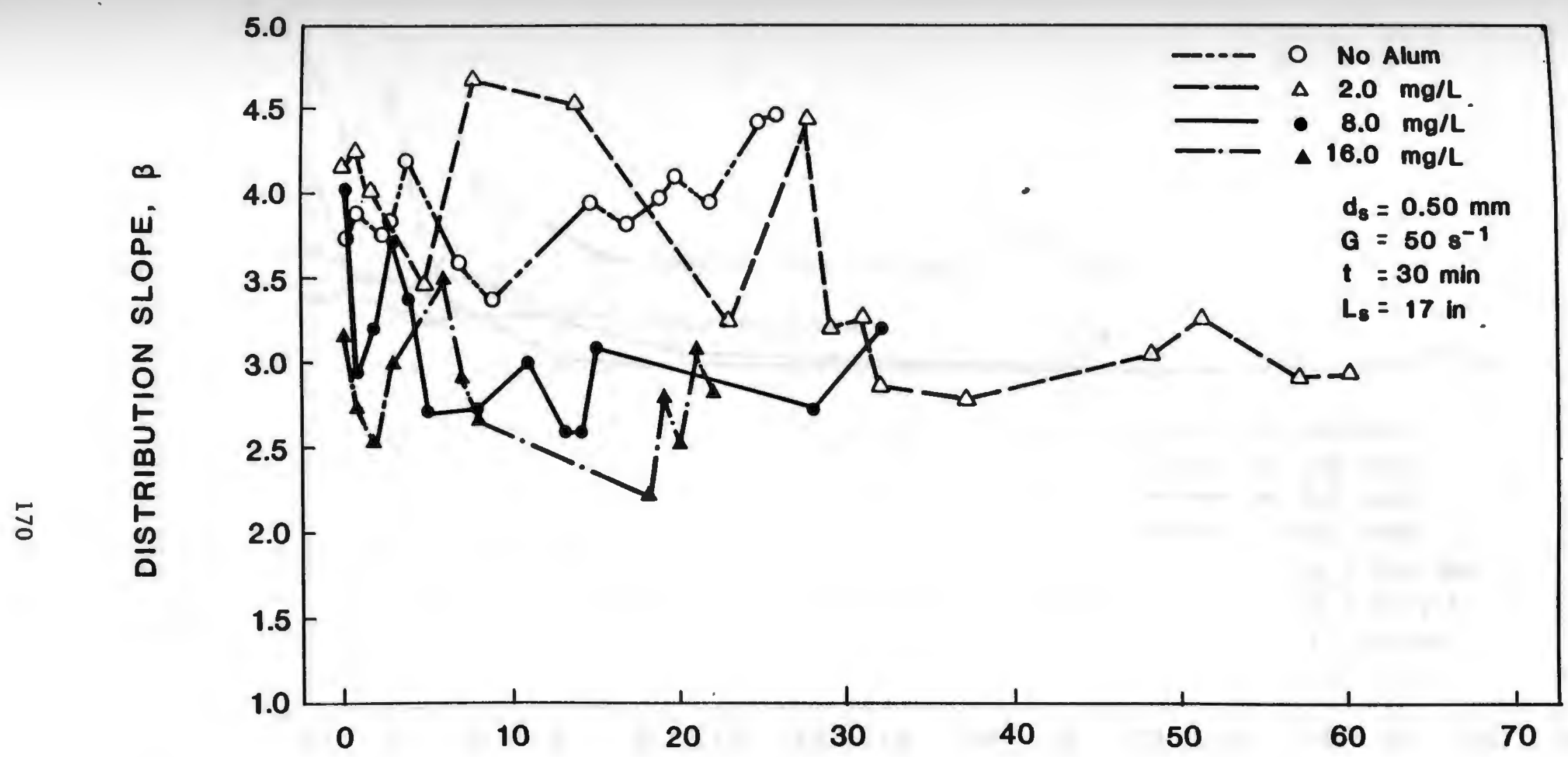

RUN TIME ( hours )

Figure 4.29 Effluent suspension distribution slope versus run time for medium sand with various alum dosages. 


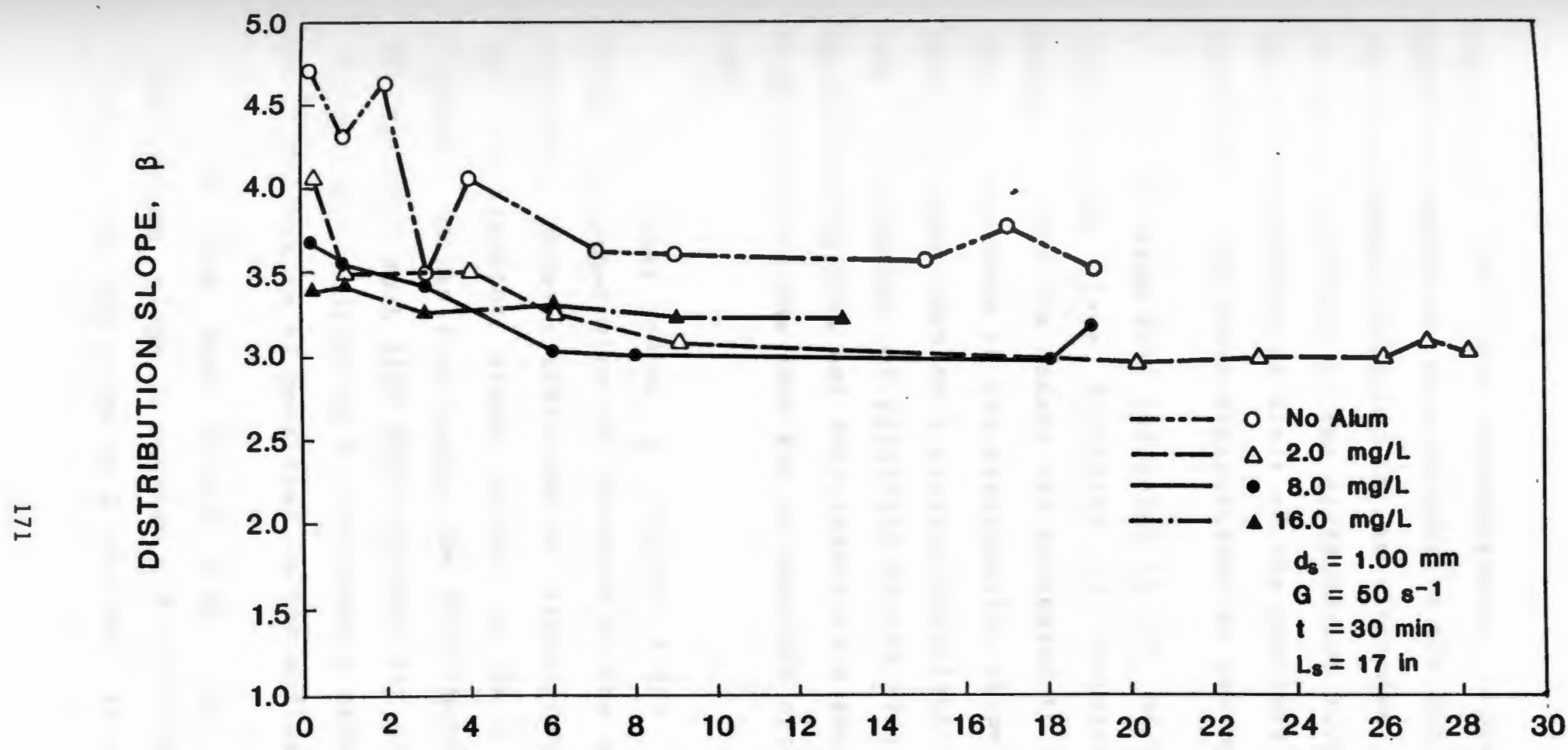

RUN TIME ( hours )

Figure 4.30 Effluent suspension distribution slope versus run time for coarse sand with varlous alum dosages. 
explanation for this inconsistency might be that the submicron particles were brought to the micro size with this low alum dosage resulting in an increase in the smaller particles and hence in the distribution slope values. Since the minimum detection limit of the particle analyzer is 2.0 micrometer, the above explanation can not be verified.

As the alum dose increased to $8.0 \mathrm{mg} / \mathrm{L}$, the effluent distribution slope decreased as compared to the lower dosages. When the dosage was increased to $16.0 \mathrm{mg} / \mathrm{L}$, there was no decrease in the distribution slope as was expected. This indicates that at a certain coagulant dose and beyond, the distribution of particles in the effluent is the same. so overdosing will not only result in a short filter run but also will give the same PSD as when the optimum alum dose is used.

For filter number 2 (Figure 4.29) the distribution slope decreased with the increase in the alum dosage as was expected. However there was no significant difference in the distribution slope values at the 0 and $2.0 \mathrm{mg} / \mathrm{L}$ alum dosages. Unlike fine media, the distribution slope values for the $16.0 \mathrm{mg} / \mathrm{L}$ alum dose influent are slightly lower than the $8.0 \mathrm{mg} / \mathrm{L}$ indicating a relationship between the media and the distribution of particles in the effluent.

With fine sand Figure 4.28 the range of the distribution slope is between $1.80-4.45$ and for medium sand (Figure 4.29) the range is 2.10-4.60. It was expected that 
for finer media the distribution slope will have higher values as compared to the coarser media in filter number 2 but this was not the case. With fine media most of the particles in the effluent are within the small size range therefore the distribution slope values are high. Since there is no significant difference between the media sizes in filter numbers 1 and $2(0.35$ versus $0.50 \mathrm{~mm})$, then the dominant removal mechanisms are known to be straining and sedimentation because of the sand size.

As the media size increases to $1.00 \mathrm{~mm}$ (Figure 4.30) the distribution slope values were high when no alum was added and decreased with time indicating that a breakthrough with coarse media is caused by larger particles. The distribution slope values are very much the same for the other alum dosages $2.0,8.0$, and $16.0 \mathrm{mg} / \mathrm{L}$. The effluent distribution slope is similar to that of the influent. This indicates that the pore spaces are large enough to allow for most of the influent particles to pass through the media without being removed. Also, an indication that suspended solids removals are not achieved by straining and sedimentation alone but by other mechanisms such as particle-particle interactions.

Unlike fine media, the coarse media gave narrower range in the values of the effluent distribution slope with all of the alum dosages used. This is because the effluent water from the coarse media had a wide range of particles in all sizes at any time during the run. Therefore, an increase or 
a decrease in the number of particles in any size interval by a few particles did not affect the distribution very much.

\subsubsection{Influence of Mixing Intensity}

The mixing intensity influences the particle size distribution in that it determines the largest floc size that can be formed. As the mixing intensity increases, smaller particles are formed giving a higher distribution slope value.

Figures $4.31,4.32$, and 4.33 are the plots of the total effluent particle count/ml versus run time for three different mixing intensities for the three filters, 1,2 , and 3 , respectively. In all three figures, the effluent quality improves with the decrease in the mixing intensity. Higher mixing intensities produce smaller flocs that tend to penetrate deeper in the bed and cause some particles to show up in the effluent. Because of particle penetration, particle to particle removal mechanism is reduced especially at the surface of the media. In this case the dominant removal mechanisms are molecular diffusion and interception. straining and sedimentation mechanisms are not so significant except with fine media. Low mixing intensities produce larger flocs that are mostly removed by straining and sedimentation mechanisms. These flocs act as a filter in which they improve the removal of particles. Therefore, the result is less particles pass through a filter when a 


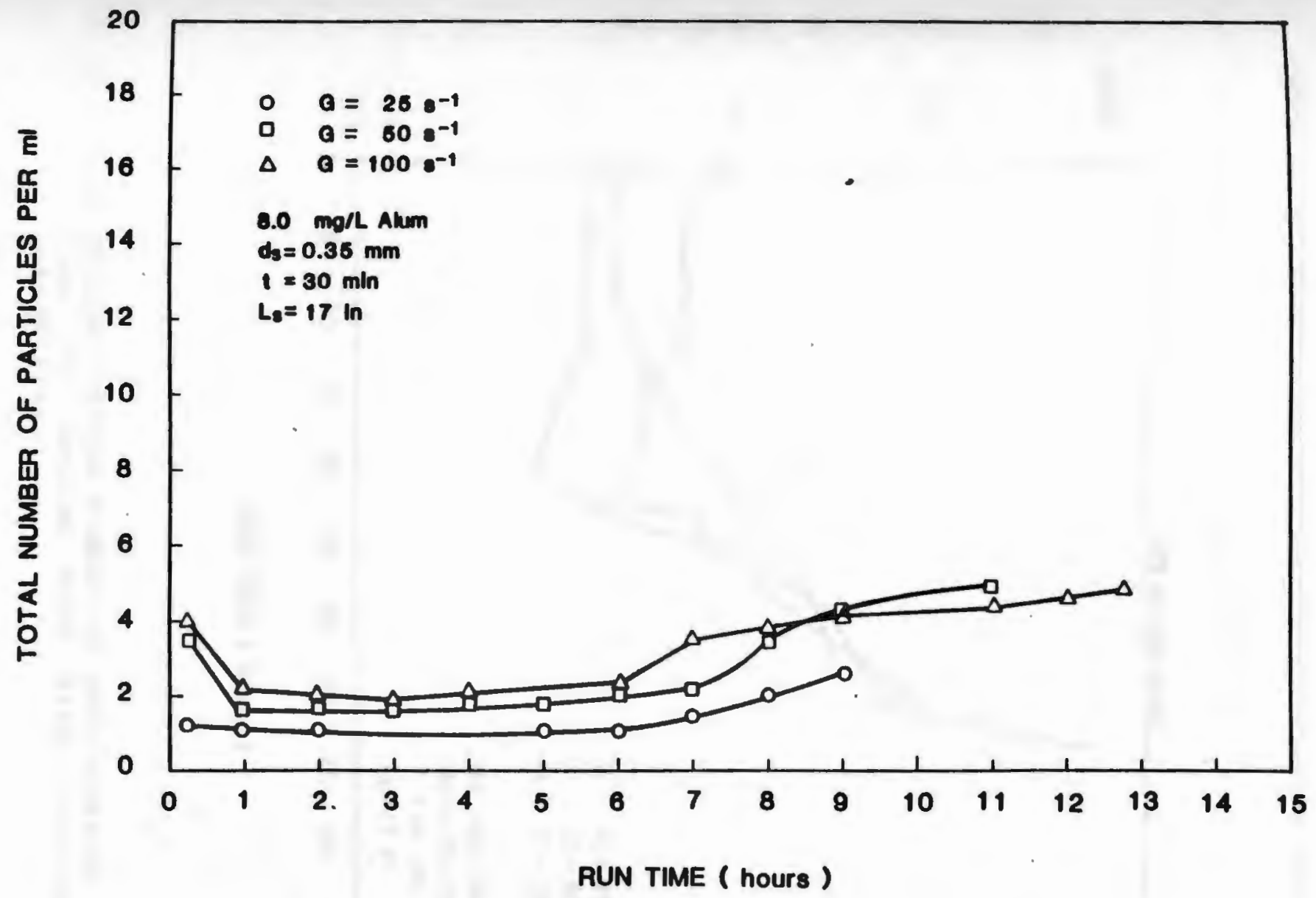

Figure 4.31 Effluent total number of particles/ml versus run time for fine sand with various mixing intensities. 


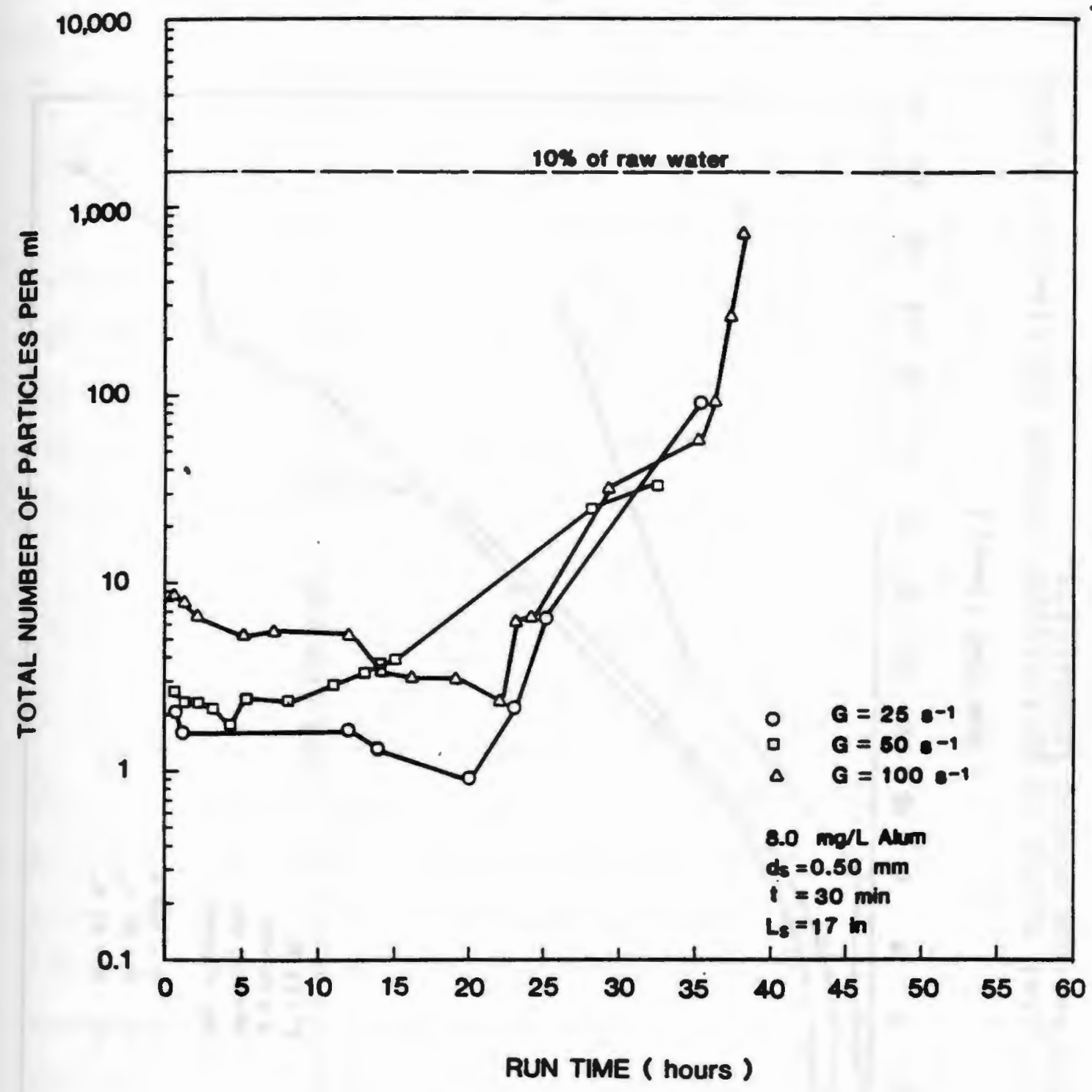

Figure 4.32 Effluent total number of particles/ml versus run time for medium sand with various mixing intensities. 


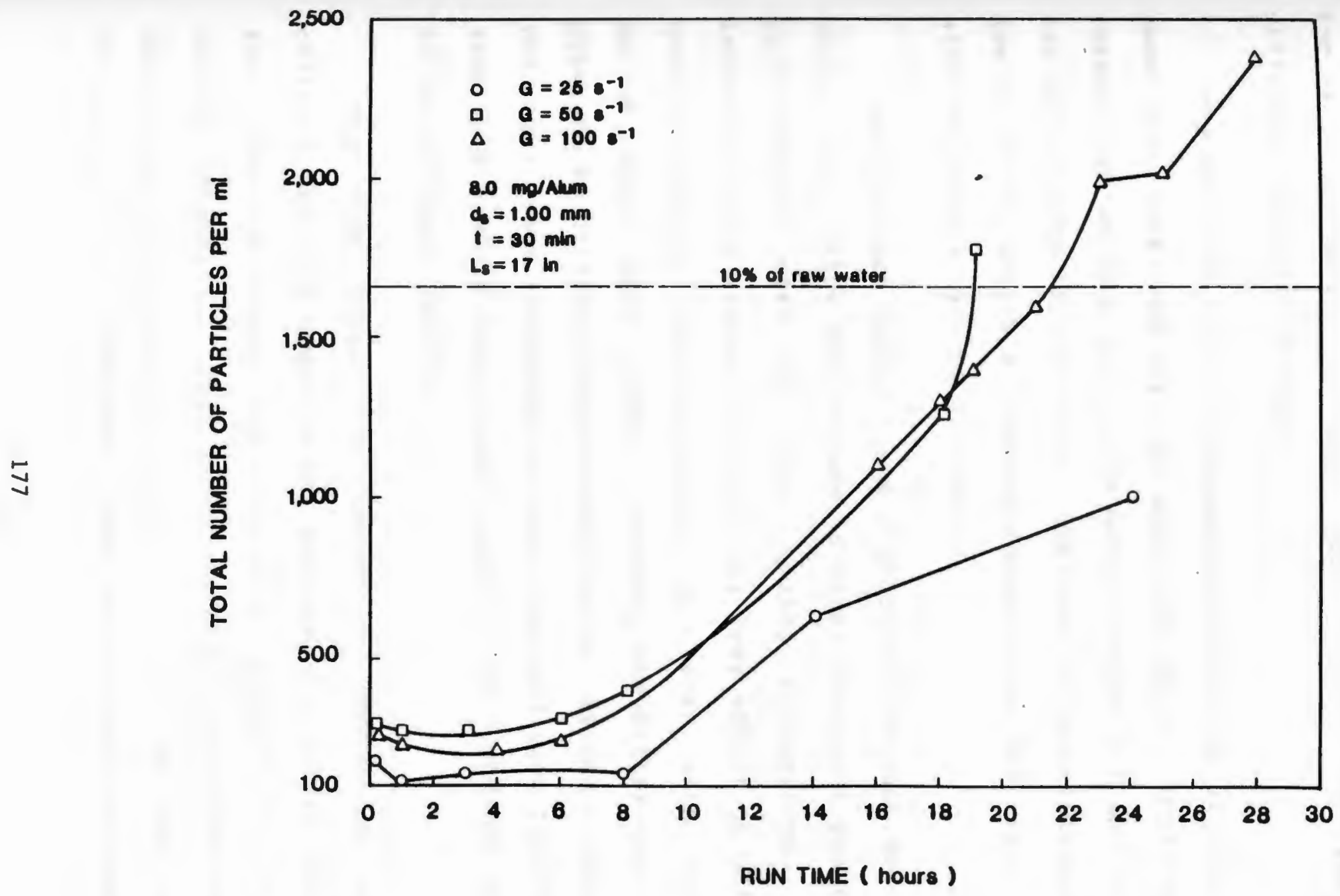

Figure 4.33 Effluent total number of particles/ml versus run time for coarse sand with various mixing intensities. 
low mixing intensity is used. In all of the three mixing intensities, the effluent total particle count was constant for a long period of time which was not the case with different coagulant dosages.

The effluent quality started deteriorating at about the same run time and with the same rate for all three mixing intensities in fine and coarse media (Tables 4.8 and 4.10 ). For medium sand (Figure 4.31), the rate of deterioration was low at the 25 and $50 \mathrm{~s}^{-1}$ mixing intensities but very high with the $100 \mathrm{~s}^{-1}$ mixing intensity.

Both filters number 1 and 2 produced effluent qualities which were below the average of water treatment plants $(10$ particles/ml) with all three mixing intensities. This indicates that mixing intensity has less effect on effluent quality than the chemical dosage, at least with fine to medium sized sand media. However, the filter run length seems to be directly proportional to the mixing intensity. The runs were terminated because the head loss reached the available head in both filter number 1 and 2 and not because of the effluent quality.

When the filter run length is controlled by the effluent particle count as was the case for filter number 3 , the filter run length was inversely proportional to the mixing intensity. This shows the relationship between the media size and the particle size in term of the removal mechanisms. It indicates that straining is an important 
removal mechanism.

The influent distribution slope, $\beta$, is expected to have a direct relationship with the mixing intensity. Figure 4.34 is a plot of the distribution slope, $\beta$, against the mixing intensity for an alum dosage of $8.0 \mathrm{mg} / \mathrm{L}$ and 30 minutes of mixing time. The 25 and $50 \mathrm{~s}^{-1}$ mixing intensities resulted in a distribution slope value of around 3.06 whereas the $100 \mathrm{~s}^{-1}$ mixing intensity gave a distribution slope value of about 3.15. Low mixing intensity results in flocs that are mostly removed by straining and sedimentation. Therefore, only smaller particles will pass through the filter bed giving high distribution slope values in the effluent. The bars around each point represent the maximum and minimum values of the distribution slope.

Plots of the distribution slope against the run time are shown in Figures $4.35,4.36$ and 4.37 for the three filters with three different mixing intensities. In Figure 4.35, the distribution slope values as was expected reached the highest for the low mixing intensity and stayed almost constant for most of the run. When the mixing intensity was increased to $50 \mathrm{~s}^{-1}$, the distribution slope decreased as was expected. However there is a great deal of fluctuation in the distribution slope values as compared to the lower mixing intensity. With a mixing intensity of $100 \mathrm{~s}-1$, the distribution slope unexpectedly rose to higher values. This is inconsistent with the hypothesis given before. 


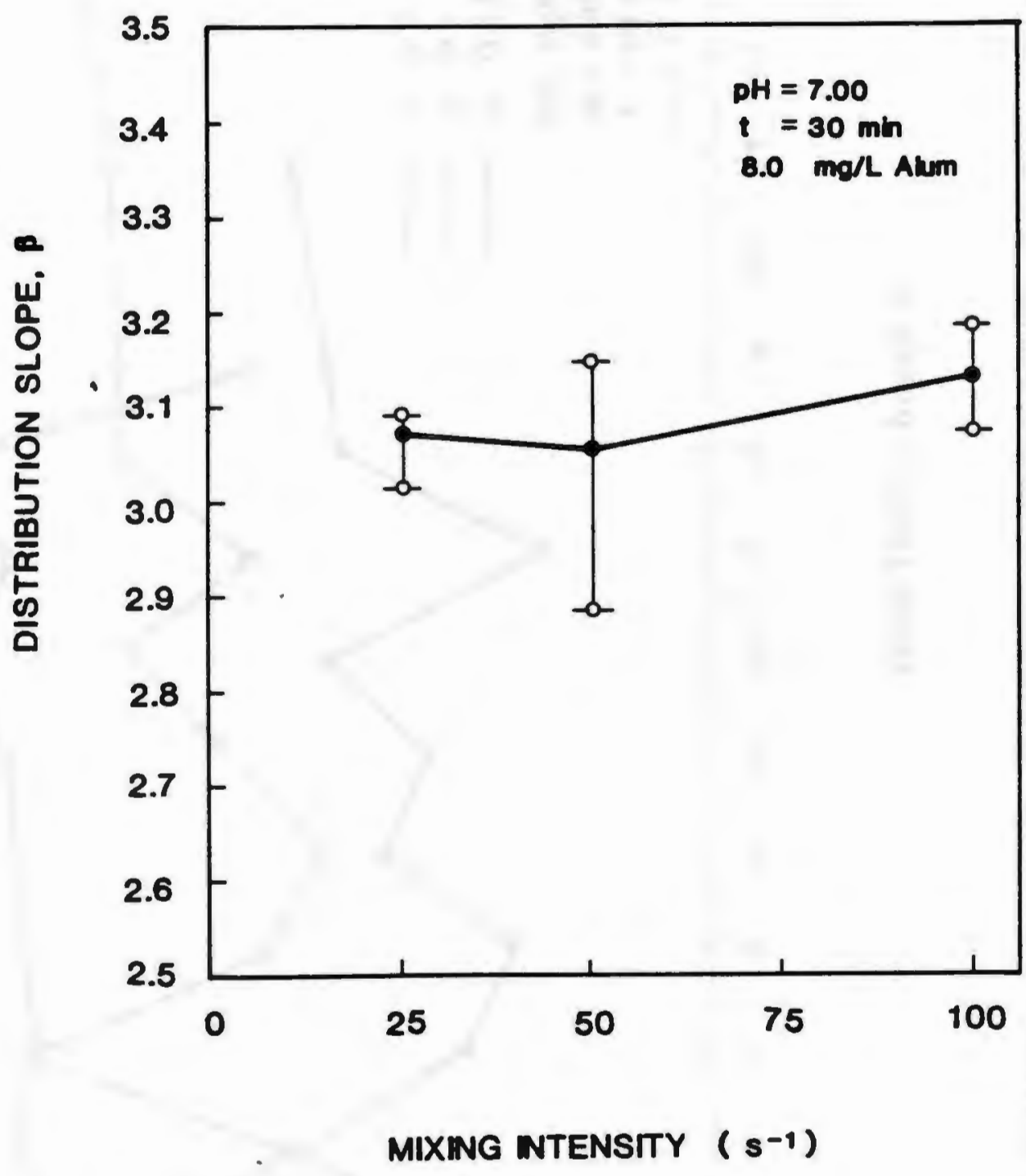

Figure 4.34 Influent suspension distribution slope versus mixing intensity for the pilot plant experiments. 


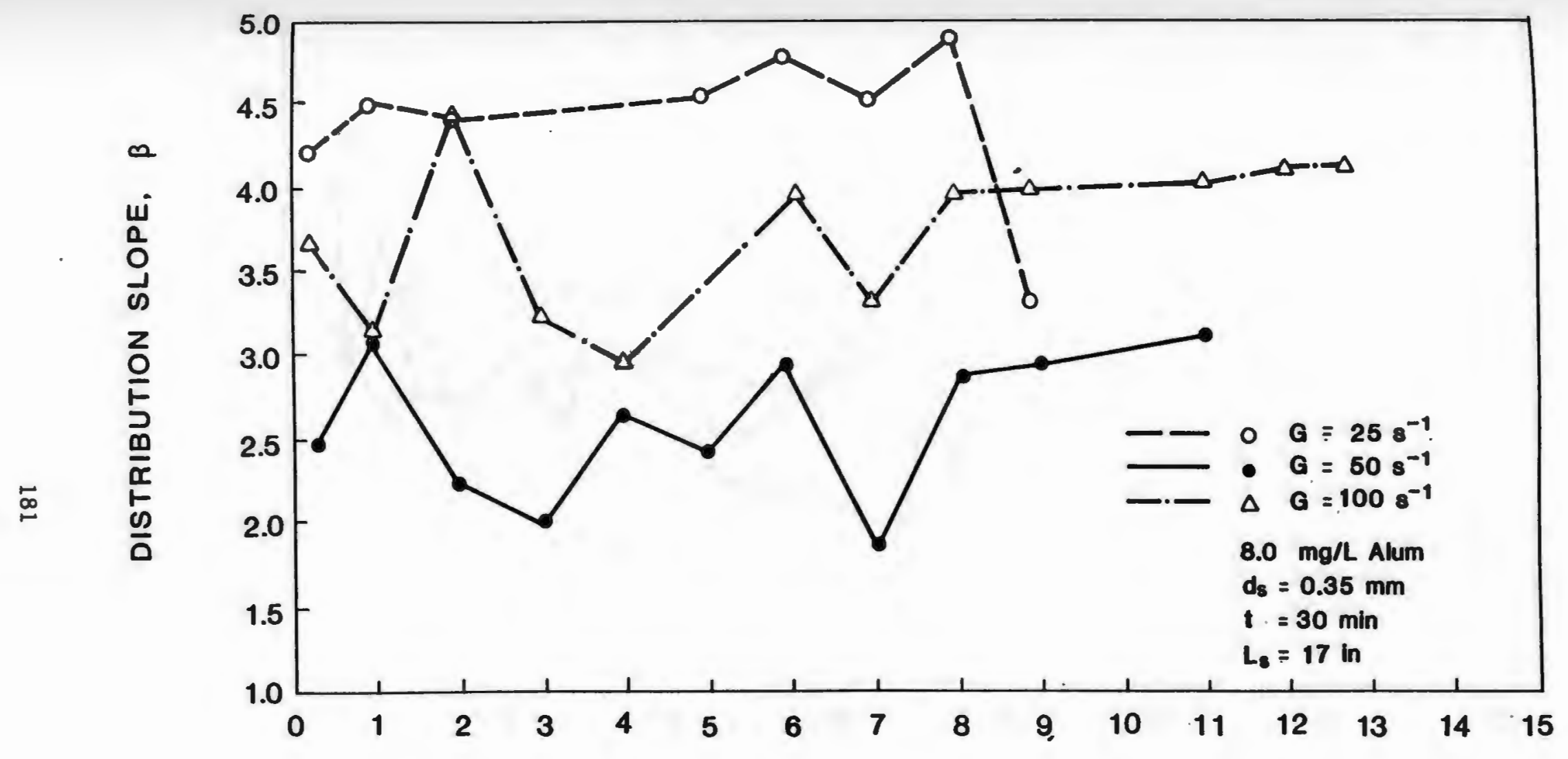

RUN TIME ( hours )

Figure 4.35 Effluent suspension distribution slope versus run time for fine sand with various mixing intensities. 


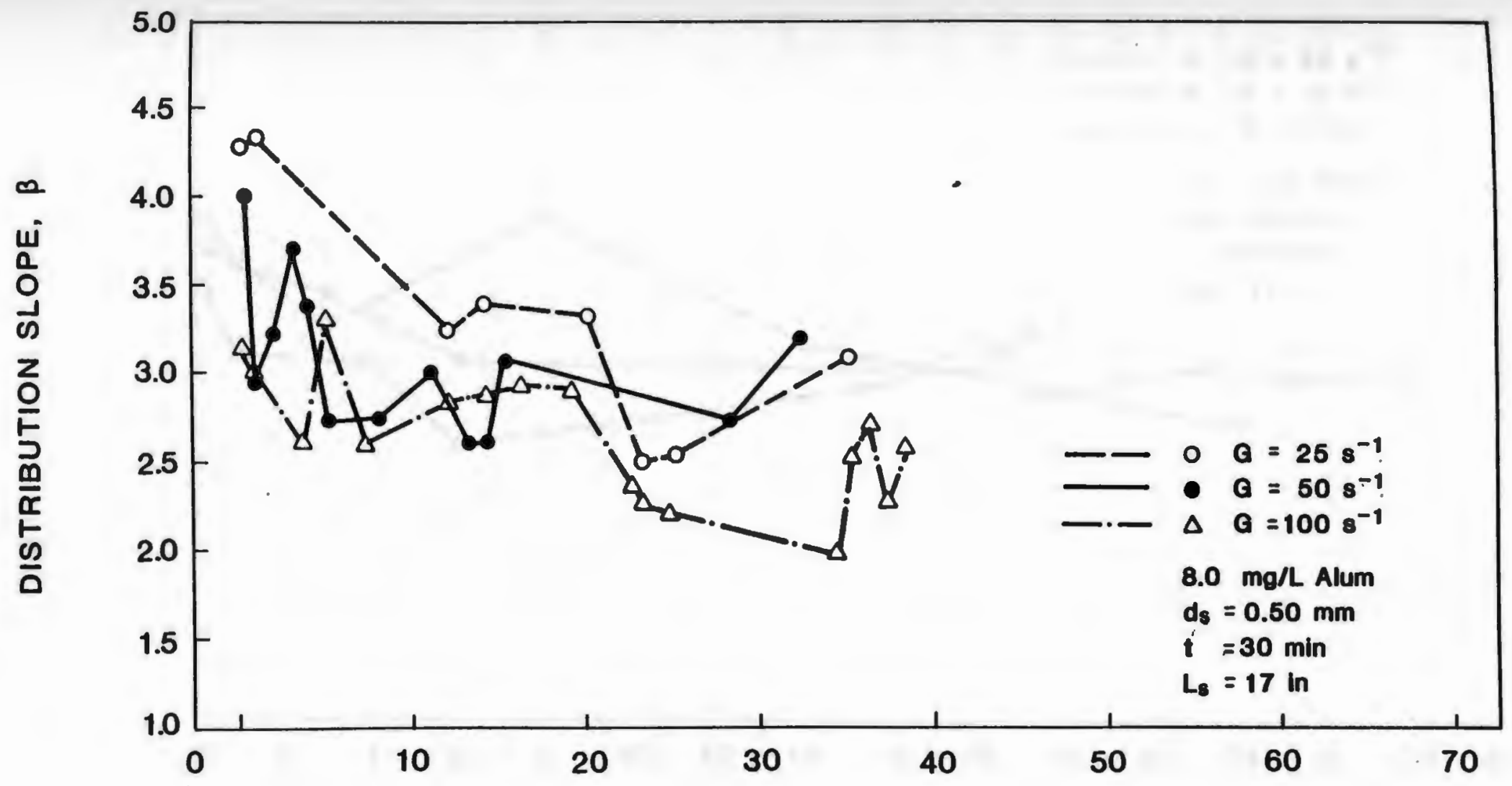

RUN TIME ( hours )

Figure 4.36 Effluent suspension distribution slope versus run time for medium sand with various mixing intensities. 


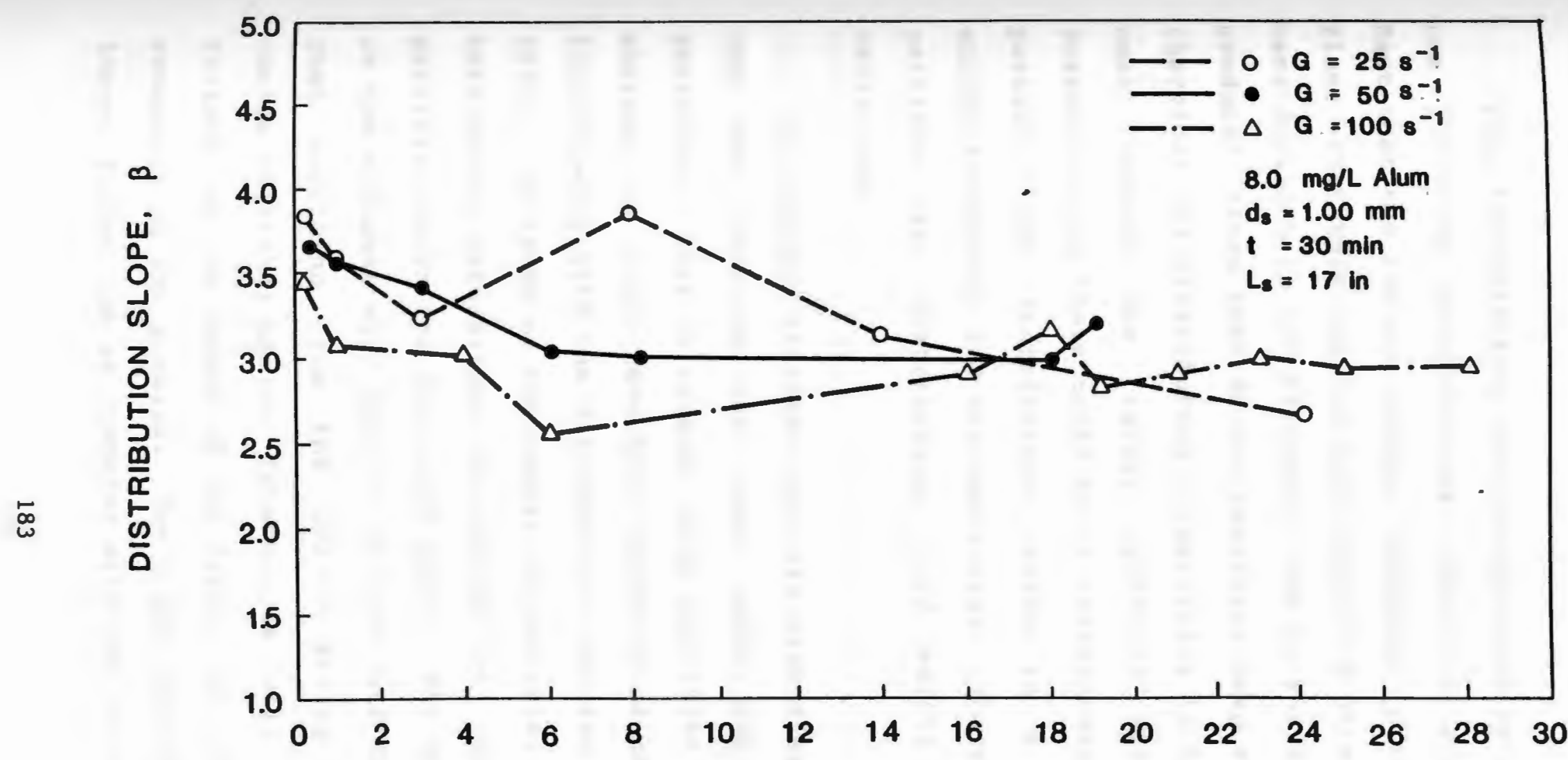

RUN TIME ( hours )

Figure 4.37 effluent suspension distribution slope versus run time for coarse sand with various mixing intensities. 
This inconsistency can be explained by one or both of the following possibilities. The first possibility is the fact that the $100 \mathrm{~s}^{-1}$ mixing intensity produced pin-point flocs that were removed with depth and only small particles were dominant in the effluent. The $50 \mathrm{~s}^{-1}$ mixing intensity produced flocs that do not penetrate deep enough in the bed therefore the distribution of particles in the effluent is more toward the larger particle sizes. The other possibility is there could be a relationship between the particle size distribution, which is a function of the mixing intensity, and the media size. Therefore, an optimum particle size distribution could exists for any specific media size.

The optimum effluent particle size distribution is the one that contains the least number and smallest size of particles. This is because large particles (greater than 15 microns in size) have been suggested as the major cause of interference with the disinfection process (Beard et al. 1977). In terms of the number of particles in the effluent, both mixing intensities ( 25 and $100 \mathrm{~s}-1$ ) gave close total particle counts for fine sand media. The dominant particles in the effluent were smaller in size with the 25 s-1 than that resulting from the $100 \mathrm{~s}^{-1}$ mixing intensities. The choice of either mixing intensities will depend on such factors as the length of the filter run achieved and on the economics of the process. The higher mixing intensity gave longer filter run as compared with the low value. 
Figure 4.36 is a plot for filter number 2 and the results match very much the hypothesis. The effluent distribution slope values decreased with the increase in the wixing intensity and with run time. The curves for the 50 $\mathrm{s}^{-1}$ and $100 \mathrm{~s}^{-1}$ mixing intensities are similar indicating that at a certain mixing intensity the effluent distribution slope starts to level off. All three mixing intensities indicate that particles in the effluent increase in size as filtration proceeds. With fine media this was not the case because the filter run was terminated before any significant effluent deterioration took place.

As the media size increases, the influence of the mixing intensity on the effluent particle size distribution does not show any significant difference between the high and low mixing intensities that were used in the study. In Figure 4.37 (filter number 3 ), the effluent distribution slope is almost identical with all three mixing intensities. The effluent distribution slope is identical to the influent distribution slope except at the early hours of operation where the former is slightly higher.

with the increase in the media size, the distribution slope region for all three mixing intensities narrows. For the fine media, the distribution slope range is 1.80-4.80, for medium sand $1.90-4.30$ and for coarse sand the range is 2.5-4.0. This is an indication of the dependance of the effluent particle size distribution on both the influent characteristics and the media size. With fine media sand, 
the effluent particle size distribution was very much dependent on the influent particle size distribution which was shown by the wide range of the distribution slope value. with coarse media, the effluent particle size distribution did not show great dependence on the influent particle size distribution except at the early filtration times.

\subsubsection{Influence of Hixing Time}

For the $0.35 \mathrm{~mm}$ media (Figure 4.38) the effluent quality improved with the increase in the mixing time. When the mixing time was 15 minutes, the initial effluent total particle count was high indicating that the small particles in the influent just passed through the sand since no previous larger flocs were available to retain them. The effluent quality improved with the run time because the deposited particles aided in the removal of the smaller ones. The effluent quality stayed relatively constant with no apparent sign of effluent deterioration. The deposited flocs on top of the media caused a rapid head loss which resulted in the termination of the filter run before effluent deterioration occurred.

As the mixing time increased to 30 minutes, the initial effluent quality improved and a longer region of constant effluent particle count was observed. Effluent deterioration is seen to take place at the end of the run. The 45 minutes mixing time gave similar effluent guality as the 30 minute mixing time. However, effluent deterioration 


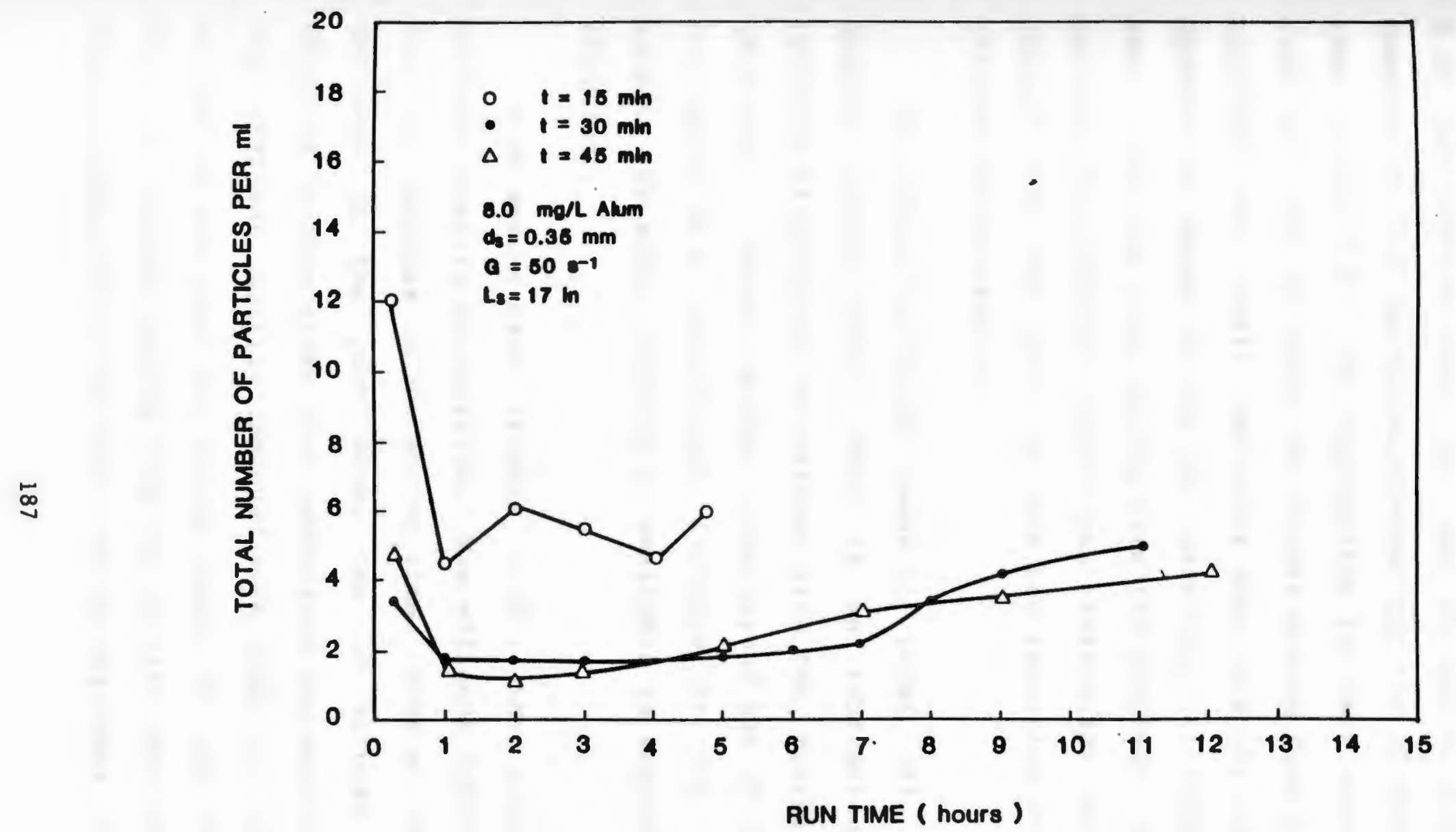

Figure 4.38 Effluent total number of particles/ml versus run time for fine sand with various mixing times. 
was not as rapid. The rate of effluent deterioration was 0.30 particles/ml-hour for the 45 minutes mixing time as compared to 0.70 particles/ml-hour for the 30 minutes mixing time (Table 4.8). The explanation for this outcome is that with this size of media, the higher mixing time (45 minutes) produced very small particles that have the capability of penetrating deeper in the bed therefore utilizing all the bed. With the lower mixing time (30 minutes), the influent particle distribution caused less penetration as a result clogged the top part of the bed resulting in more rapid effluent deterioration.

The filter run length seems to level off at the 45 minutes mixing time. This is an indication that floc breakage is reaching the maximum with the operating mixing intensity. Excess mixing time beyond the 45 minutes will not result in a significant increase in the filter run length. No other evidence is available to support the above statement.

With medium sand (Figure 4.39), the same trend in effluent quality is observed. The effluent quality improved with the increase in the mixing time. However, due to the increase in the pore size, the 15 minutes mixing time resulted in floc sizes that penetrated the sand bed in which the effluent quality improved with time to a level similar to that of the other two mixing times, 30 and 45 minutes. with 15 minutes mixing time the initial particle count was high as compared to the other two mixing times $(15.5$ versus 


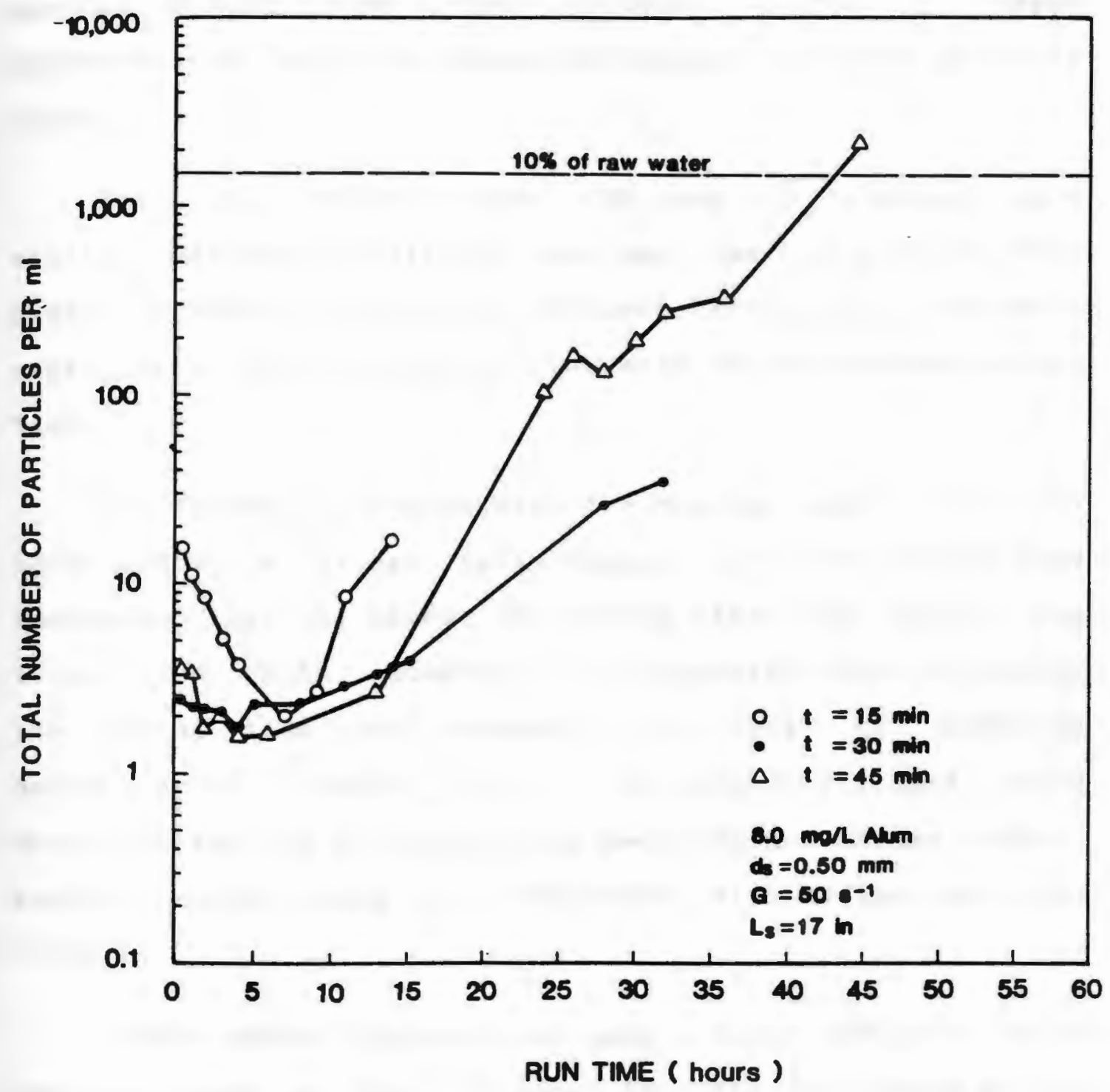

Figure 4.39 Effluent total number of particles/ml versus run time for medium sand with various mixing times. 
2.5 and 3.5 particles/ml). With this size of media and 15 minutes mixing time the effluent showed a rapid deterioration with no region of constant effluent particle count.

The higher mixing times ( 30 and 45 minutes) gave similar effluent qualities as was observed with the fine media. However, the rate of effluent deterioration was more rapid with the 45 minutes than with the 30 minutes mixing time.

The filter run length with the medium sand $(0.50 \mathrm{~mm})$ have almost a linear relationship with the mixing time indicating that the higher the mixing time the longer the filter run length. However, it is expected that the higher the mixing time the steeper the rate of effluent deterioration (Table 4.9). To avoid effluent rapid deterioration and to provide the best maximum volume output, medium mixing times are desirable with medium sand size filters.

Coarse media (Figure 4.40) gave a lower effluent total particle count for the 15 minutes than the 30 minutes mixing time. The region of constant effluent particle count increased but the deterioration was as rapid as before. With a low mixing time, the flocs that have been formed have penetrated the sand bed and were removed mostly by sedimentation on media grains. 


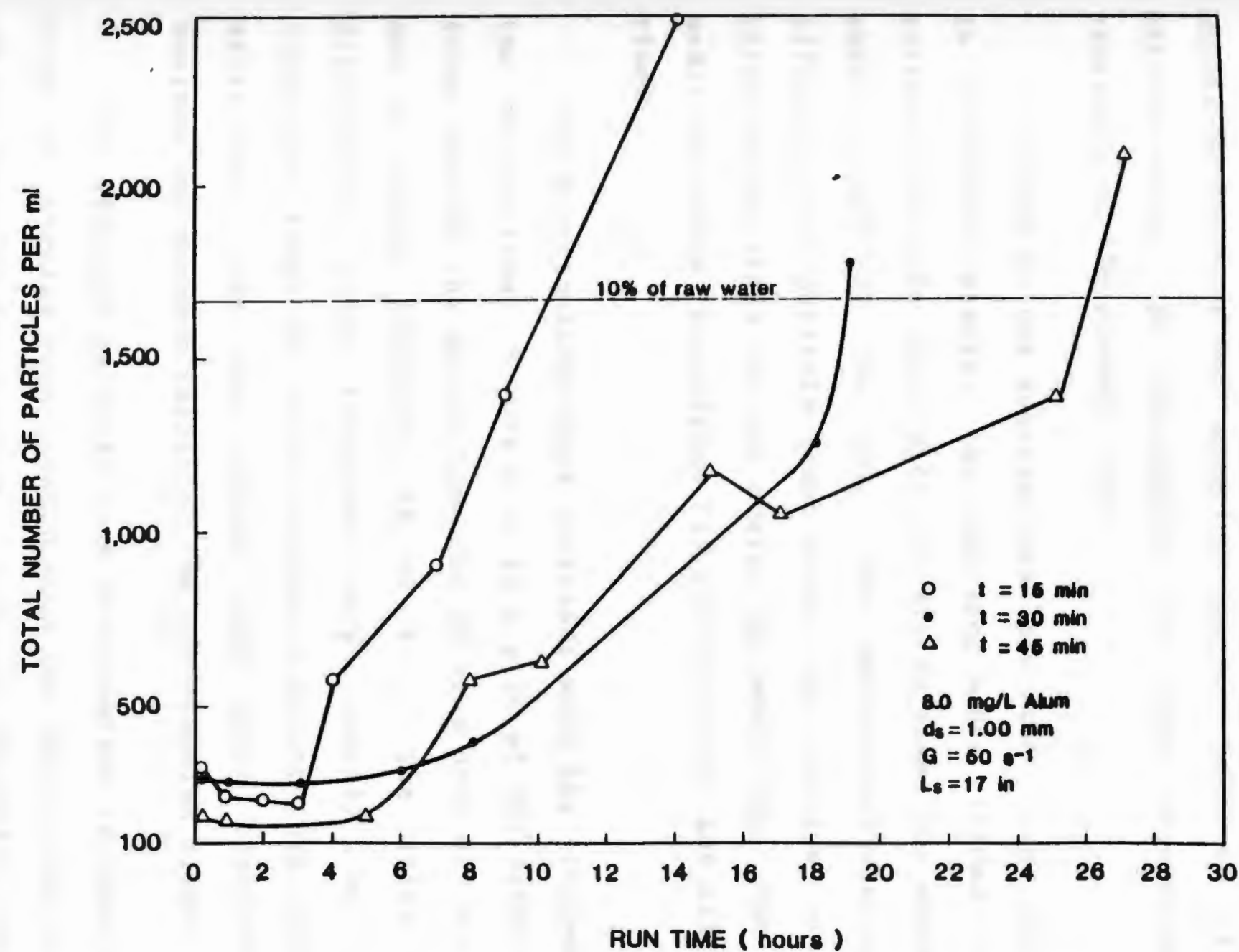

Figure 4.40 Effluent total number of particles/ml versus run time for coarse sand with various mixing times. 
Unlike fine and medium sand, the effluent particle count from the coarse media showed improvement with the 45 minute mixing time but with a similar rate of effluent deterioration. No explanation for this observation is available at the present time.

Compared to the average total particle count achieved in treatment plants, the fine sand media yielded a better effluent particle count with all mixing times for almost the whole length of the run. The medium sand also gave an effluent total particle count below the average with all three mixing times but not during the whole run. The coarse media was above the average ( 10 particles/ml) for all mixing times.

The distribution slope increases with the increase in the mixing time. Figure 4.41 is a plot of the distribution slope against the mixing time for an alum dose of $8.0 \mathrm{mg} / \mathrm{L}$ and a mixing intensity of $50 \mathrm{~s}^{-1}$. The value of the distribution slope increases very rapidly as mixing increased from 15 to 30 minutes of mixing then levels off after that. The bars around each point represent the maximum and minimum values of the distribution slope.

The effluent particle size distribution is expected to have an inverse relationship with the mixing time in terms of the distribution slope, $\beta$. However, the media size could alter this generalized rule because of factors such as the pore space available for particle deposition. With fine 


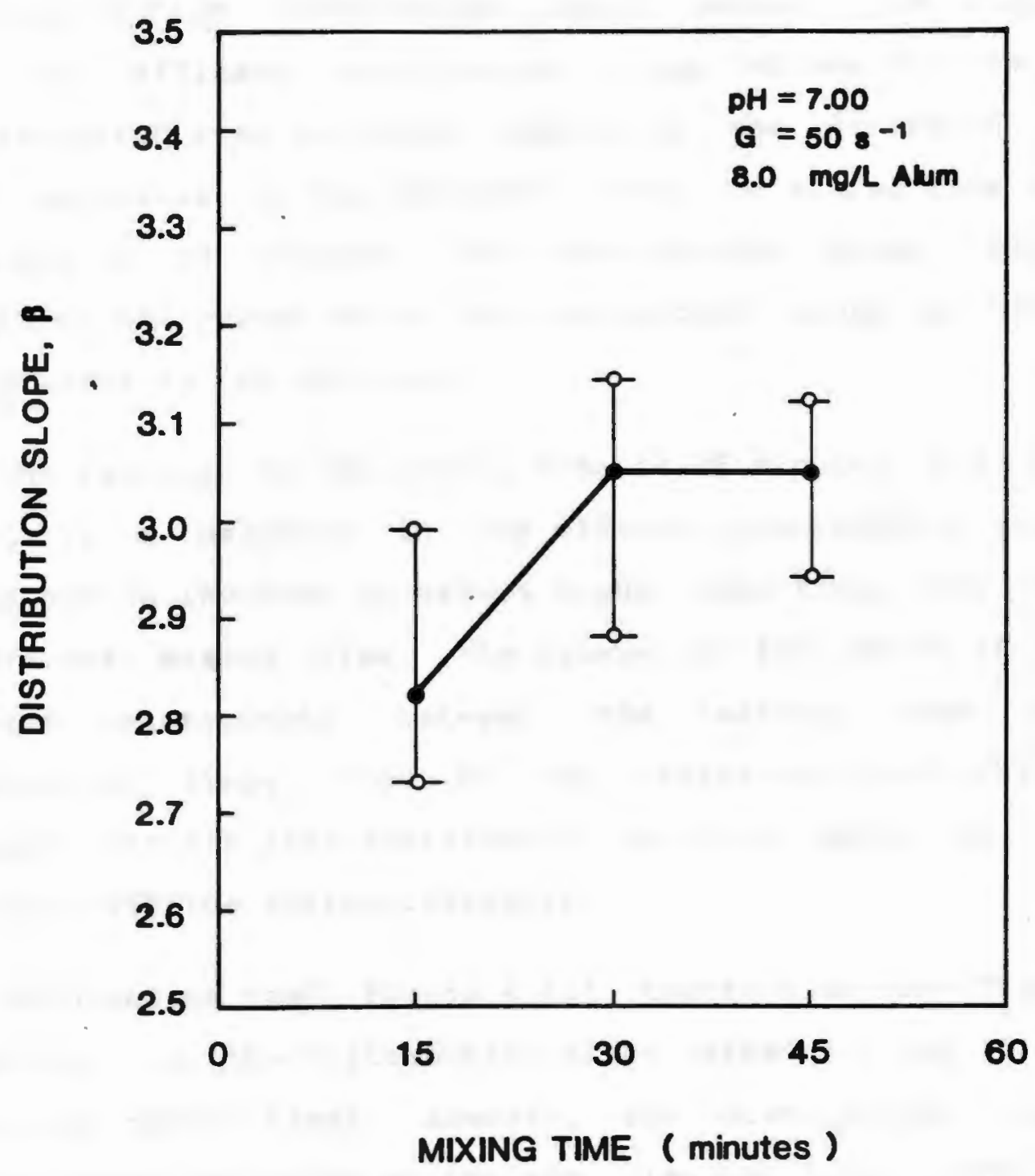

Figure 4.41 Influent suspension distribution slope versus mixing time for the pilot plant experiments. 
sand, removal is mostly achieved by straining when large flocs are present in the influent suspension. Therefore, small particles are expected to dominate in the effluent resulting in high distribution slope values. In Figure 4.42, the effluent distribution slope values for the 15 minutes mixing time are high indicating the dominance of small particles in the effluent. When the mixing time was increased to 30 minutes, the distribution slope values dropped to the lowest value indicating that larger particles were present in the effluent.

The increase in the mixing time to 45 minutes did not result in a decrease in the effluent distribution slope values but an increase to values higher than those for the 30 minutes mixing time. The reason for this shift in the expected relationship between the mixing time and distribution slope could be the existence of an optimum influent particle size distribution for fine media as was the case with the mixing intensity.

with medium sand (Figure 4.43), there is no significant difference in the distribution slope values for the 15 and 30 minutes mixing times. However, the distribution slope values start declining at the same time the total number of particles in the effluent starts inclining. This is an indication that the effluent deterioration associated with medium sand is the result of breakthrough of larger particles. 


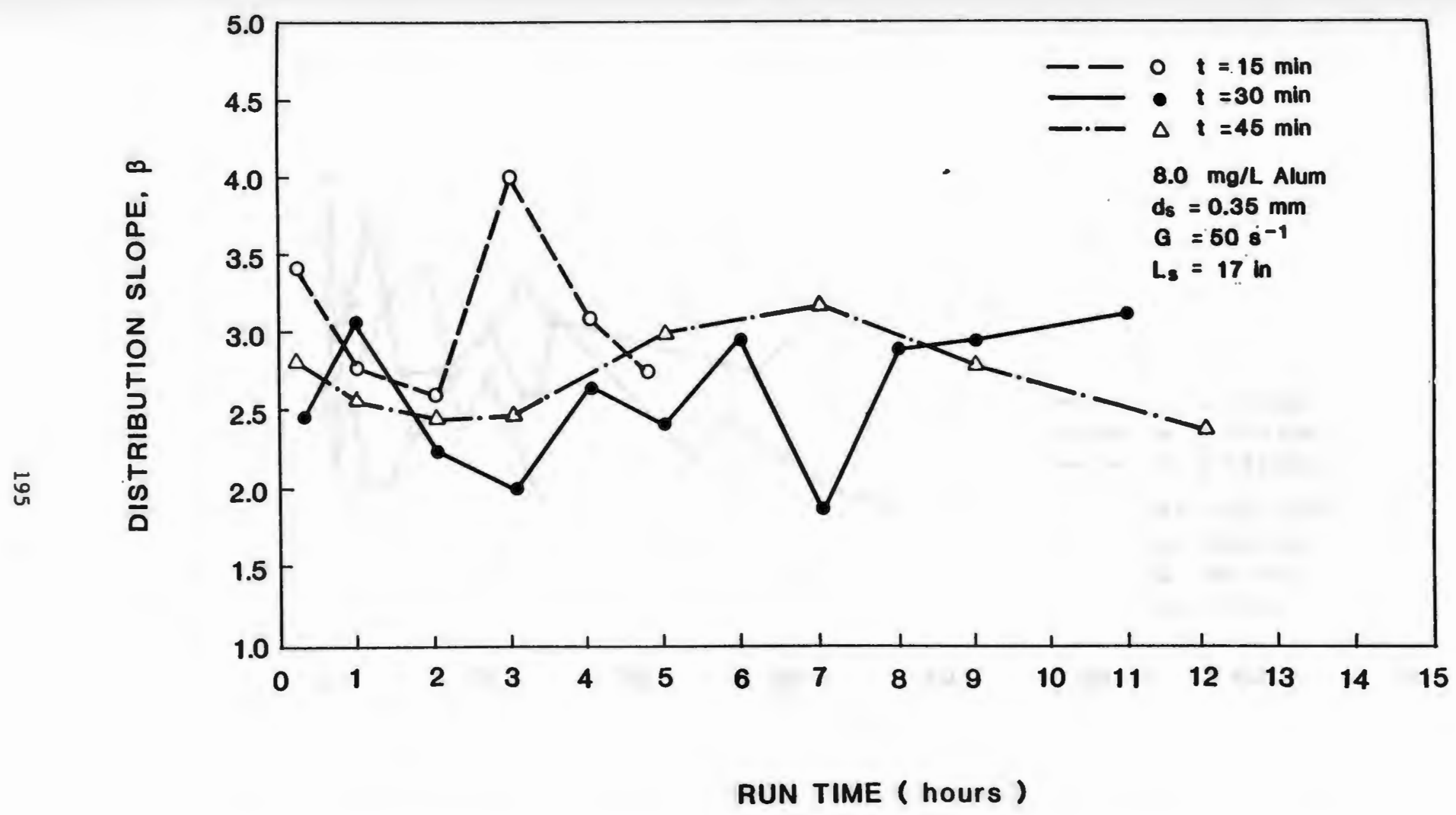

Figure 4.42 Effluent suspension distribution slope versus. run time for fine sand with various mixing times. 


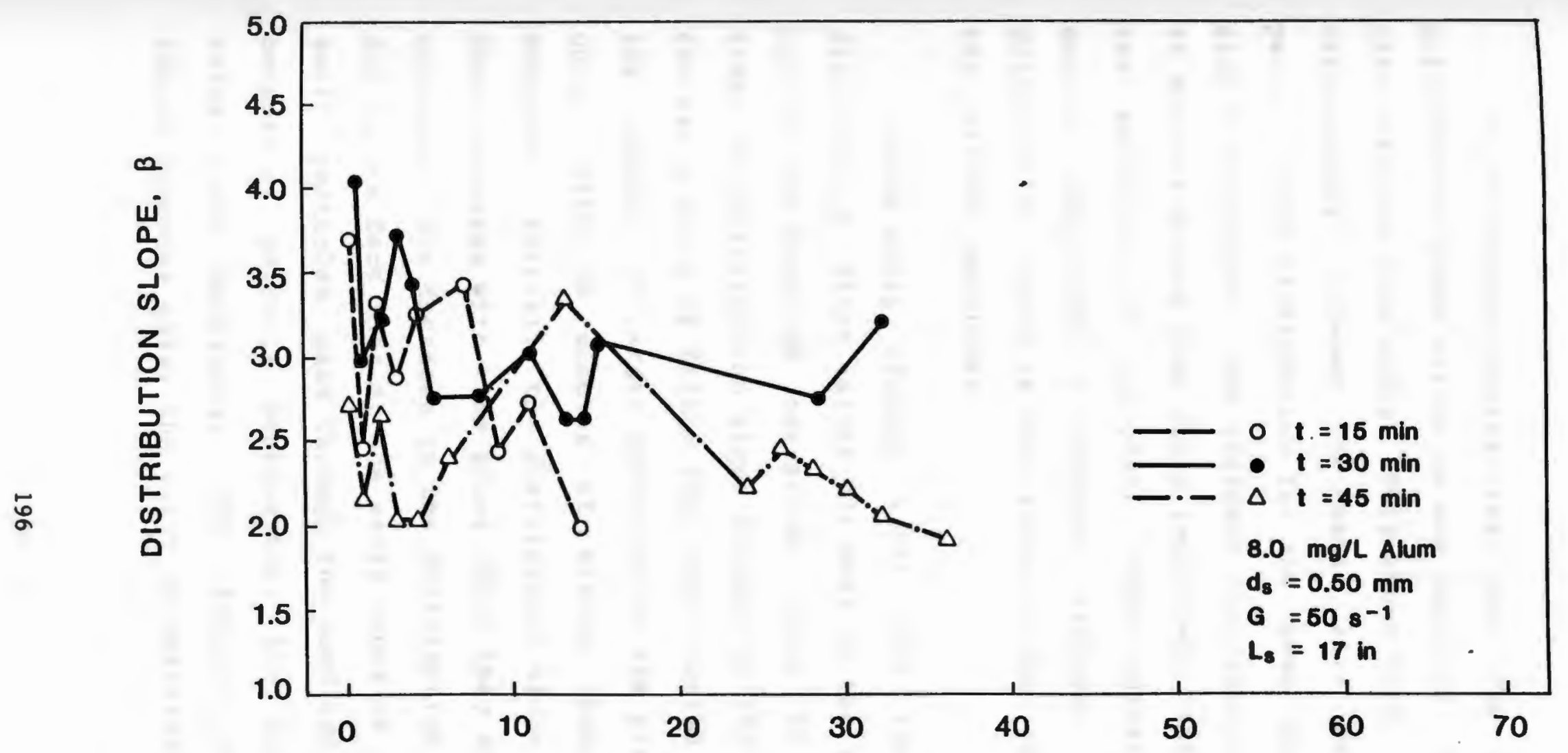

RUN TIME ( hours )

Figure 4.43 Effluent suspension distribution slope versus run time for medium sand with various mixing times. 
The 45 minutes mixing time gave the lowest effluent distribution slope values as was expected. This was not the case with the fine media. This shows that there exists a relationship between the media size and the effluent particle size distribution for the same influent particle size distribution. The effluent distribution slope with the 45 minutes mixing time shows relatively constant values with the exception of one point. This indicates that a higher mixing time gives a uniform effluent particle size distribution which is the result of deep penetration of all the influent particles.

Coarse media (Figure 4.44) gave identical effluent distribution slope values for most of the run-except at the initial few hours of operation. With 15 minutes mixing time, the distribution slope dropped to the lowest values at the early hours of filtration, then started rising due to the removal of larger particles by the previously retained ones. With 30 minutes of mixing time, the opposite occurred. Initially the distribution slope values were high then decreased with time after which they stayed relatively constant. The decrease in the distribution slope values was due to the fact that at the early hours of filtration only small particles pass through the sand bed, and, with time, because of particle penetration, the distribution slope value start declining. The effluent distribution slope stayed constant after the start of deterioration. 


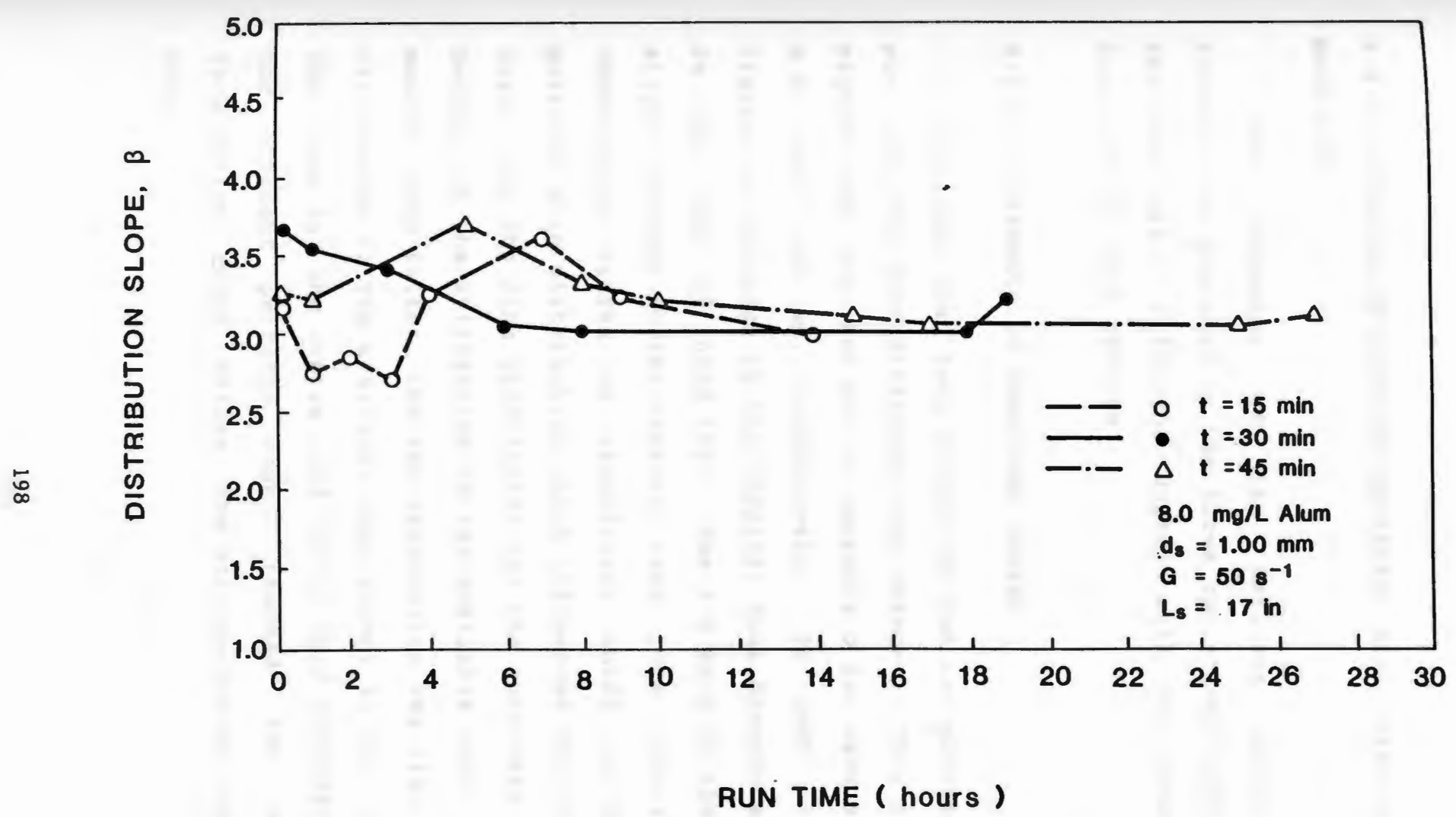

Figure 4.44 Effluent suspension distribution slope versus run time for coarse sand with various mixing times. 
4.2.2 Influence of Influent Particle size Distribution on Head Loss

The influence of the influent particle size distribution produced by the three operational parameters on the head loss at different depths will be presented and discussed in this section.

\subsubsection{Influence of Coagulant Dosage}

The tbtal head loss across the bed is plotted against run time for four different alum dosages. This is shown in Figures $D-1, D-2$, and $D-3$ in Appendix $D$ for sand sizes 0.35 , 0.5 , and $1.00 \mathrm{~mm}$, respectively. In each of the three figures an increase in the chemical dose brought an increase in the rate of head loss. The $2.0 \mathrm{mg} / \mathrm{L}$ of alum showed a slight increase in the rate of head loss indicating that underdosing causes no significant shift in the influent particle size distribution which influences the rate of head loss. For the fine sand filter all the runs were terminated because of the utilization of the available head. For the medium sand filter, the run termination was also due to the utilization of the available head except in two runs. All the runs for the coarse sand filter were terminated because the effluent particle count reached the established termination count before the utilization of the available head. 
The best fit for the curves in Figures $D-1, D-2$, and D-3 was found to be a linear regression with a coefficient of determination of 0.98 and above (Tables 4.11, 4.12, and 4.13). This implies that the deposition of particles is within the bed (in depth filtration)for all the dosages used or the contribution of the initial readings to the line are insignificant therefore the best fit is a linear type instead of an exponential. It was expected that as the coagulant dosage increased, the size and mass of solids are also incteased, therefore more particles would be deposited on top of the media resulting in an exponential type of relationship instead of linear. A mat of solids was observed to form on top of the sand media which tended to increase in thickness with an increase in the coagulant dosage and decreases in thickness with an increase in media size. When the removal of particles is achieved by straining alone, then the head loss is expected to have an exponential profile with run time. When the removal of particles is within the sand bed (in depth filtration) then the head loss profile is expected to have a linear relationship with run time.

The slope of the curves in Figures $D-1, D-2$, and $D-3$ is the rate of head loss in inches/hour. These rates for the different depths are shown in Tables $E-1, E-2$, and $E-3$ (Appendix E) for filters number 1, 2, and 3, respectively. Those same rates are plotted in Figures 4.45, 4.46, and 4.47. With fine media (Figure 4.45), most of the head loss 
Table 4.11- Coefficients of Determination for the Rates of Bead Loss for Filter No. 1

\begin{tabular}{|c|c|c|c|c|c|}
\hline $\begin{array}{l}\text { Influent } \\
\text { Type }\end{array}$ & 1 & $\underset{5}{\text { Sand }}$ & Depth (inches) & 13 & 17 \\
\hline $0 \mathrm{mg} / \mathrm{L}$ Alum & 0.9980 & 0.9990 & 0.9990 & 0.9990 & 0.9990 \\
\hline $2 \mathrm{mg} / \mathrm{L}$ Alum & 0.9990 & 0.9990 & 0.99 .90 & 0.9990 & 0.9990 \\
\hline $8 \mathrm{mg} / \mathrm{L}$ Alum & 0.9960 & 0.9940 & 0.9940 & 0.9940 & 0.9940 \\
\hline $16 \mathrm{mg} / \mathrm{L}$ Alum & 0.9996 & 0.9980 & 0.9996 & 0.9996 & 0.9990 \\
\hline$G=25 \mathrm{~s}^{-1}$ & 0.9996 & 0.9998 & 0.9998 & 0.9998 & 0.9998 \\
\hline$G=50 \mathrm{~s}^{-1}$ & 0.9960 & 0.9940 & 0.9940 & 0.9940 & 0.9940 \\
\hline$G=100 s^{-1}$ & 0.9964 & 0.9956 & 0.9956 & 0.9958 & 0.9956 \\
\hline$t=15 \min$ & 0.9934 & 0.9924 & 0.9926 & 0.9926 & 0.9926 \\
\hline$t=30 \mathrm{~min}$ & 0.9960 & 0.9940 & 0.9940 & 0.9940 & 0.9940 \\
\hline$t=45 \mathrm{~min}$ & 0.9932 & 0.9924 & 0.9926 & 0.9930 & 0.9934 \\
\hline
\end{tabular}


Table 4.12- Coefficients of Determination for the Rates of Head Loss for Filter No. 2

\begin{tabular}{|c|c|c|c|c|c|}
\hline $\begin{array}{l}\text { Influent } \\
\text { Type }\end{array}$ & 1 & $\begin{array}{l}\text { Sand } \\
5\end{array}$ & Depth (inches) & 13 & 17 \\
\hline $0 \mathrm{mg} / \mathrm{L}$ Alum & 0.9864 & 0.9882 & 0.9892 & 0.9896 & 0.9896 \\
\hline $2 \mathrm{mg} / \mathrm{L}$ Alum & 0.9966 & 0.9960 & 0.9942 & 0.9954 & 0.9936 \\
\hline $8 \mathrm{mg} / \mathrm{L}$ Alum & 0.9936 & 0.9803 & 0.9791 & 0.9787 & 0.9783 \\
\hline $16 \mathrm{mg} / \mathrm{L}$ Alum & 0.9962 & 0.9910 & 0.9894 & 0.9890 & 0.9857 \\
\hline$G=25 \mathrm{~s}^{-1}$ & 0.9992 & 0.9922 & 0.9904 & 0.9894 & 0.9890 \\
\hline$G=50 \mathrm{~s}^{-1}$ & 0.9936 & 0.9803 & 0.9791 & 0.9787 & 0.9783 \\
\hline$G=100 \mathrm{~s}^{-1}$ & 0.9986 & 0.9870 & 0.9843 & 0.9839 & 0.9825 \\
\hline$t=15 \min$ & 0.9986 & 0.9976 & 0.9976 & 0.9976 & 0.9978 \\
\hline$t=30 \mathrm{~min}$ & 0.9936 & 0.9803 & 0.9791 & 0.9787 & 0.9783 \\
\hline$t=45 \mathrm{~min}$ & 0.9934 & 0.9841 & 0.9845 & 0.9847 & 0.9849 \\
\hline
\end{tabular}


Table 4.13-Coefficient of Determination for the Rate of Head Loss for Filter No. 3

\begin{tabular}{|c|c|c|c|c|c|}
\hline $\begin{array}{l}\text { Influent } \\
\text { Type }\end{array}$ & 1 & $\underset{5}{\text { Sand }}$ & Depth ${ }_{9}^{\text {(inches) }}$ & 13 & 17 \\
\hline $0 \mathrm{mg} / \mathrm{L}$ Alum & 0.9423 & 0.9524 & 0.9469 & 0.9580 & 0.9553 \\
\hline $2 \mathrm{mg} / \mathrm{L}$ Alum & 0.9954 & 0.9966 & 0.9958 & 0.9980 & 0.9980 \\
\hline $8 \mathrm{mg} / \mathrm{L}$ Alum & 0.9982 & 0.9960 & 0.9948 & 0.9948 & 0.9942 \\
\hline $16 \mathrm{mg} / \mathrm{L}$ Alum & 0.9988 & 0.9990 & 0.9994 & 0.9994 & 0.9998 \\
\hline$G=25 \mathrm{~s}^{-1}$ & 0.9960 & 0.9956 & 0.9906 & 0.9904 & 0.9908 \\
\hline$G=50 s^{-1}$ & 0.9982 & 0.9960 & 0.9948 & 0.9948 & 0.9942 \\
\hline$G=100 s^{-1}$ & 0.9946 & 0.9946 & 0.9960 & 0.9948 & 0.9944 \\
\hline$t=15 \mathrm{~min}$ & 0.9964 & 0.9990 & 0.9970 & 0.9974 & 0.9978 \\
\hline$t=30 \mathrm{~min}$ & 0.9982 & 0.9960 & 0.9948 & 0.9948 & 0.9942 \\
\hline$t=45 \mathrm{~min}$ & 0.9976 & 0.9932 & 0.9894 & 0.9896 & 0.9900 \\
\hline
\end{tabular}




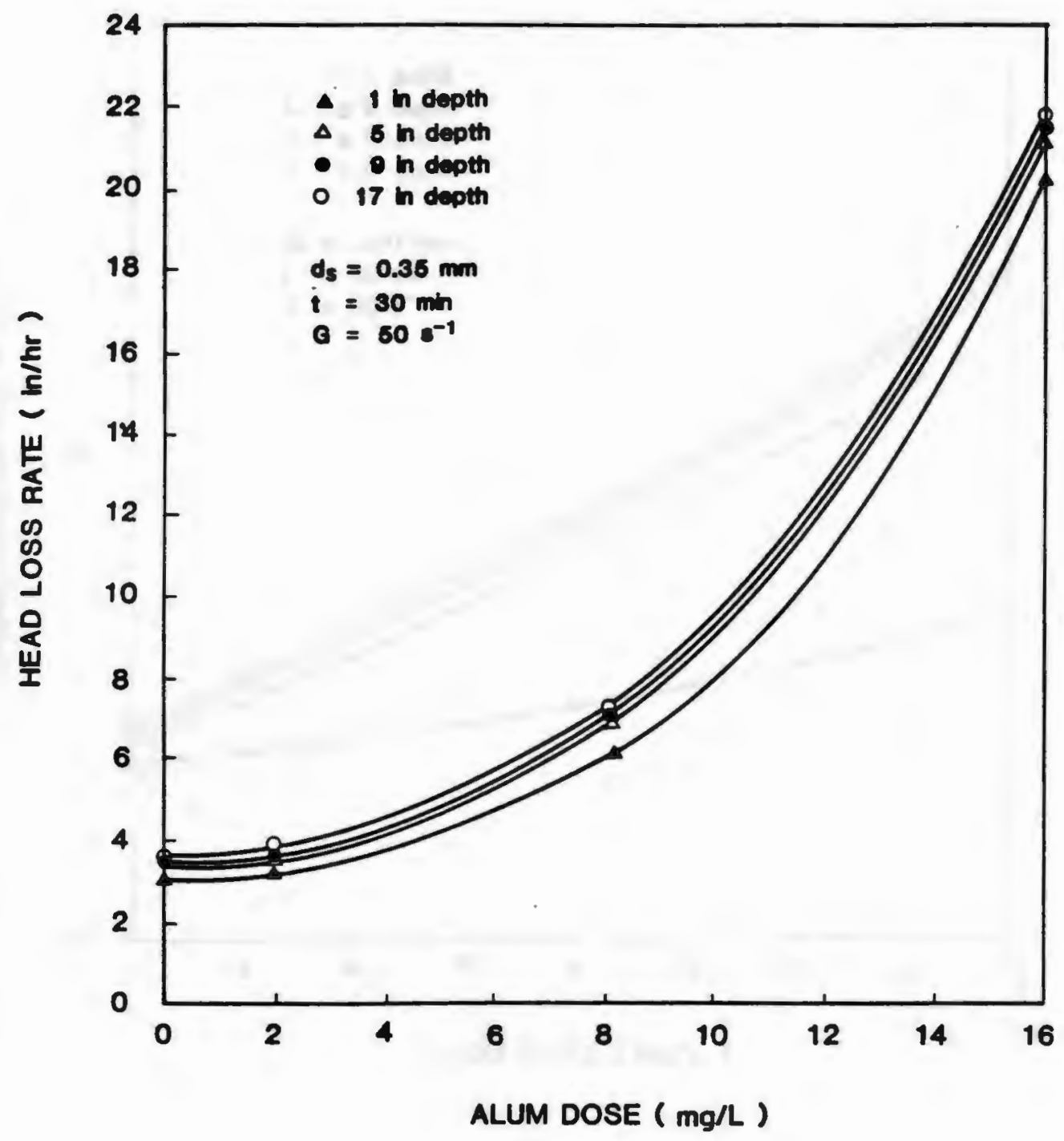

Figure 4.45 Rate of head loss development versus alum dose for fine media at various sand depths. 


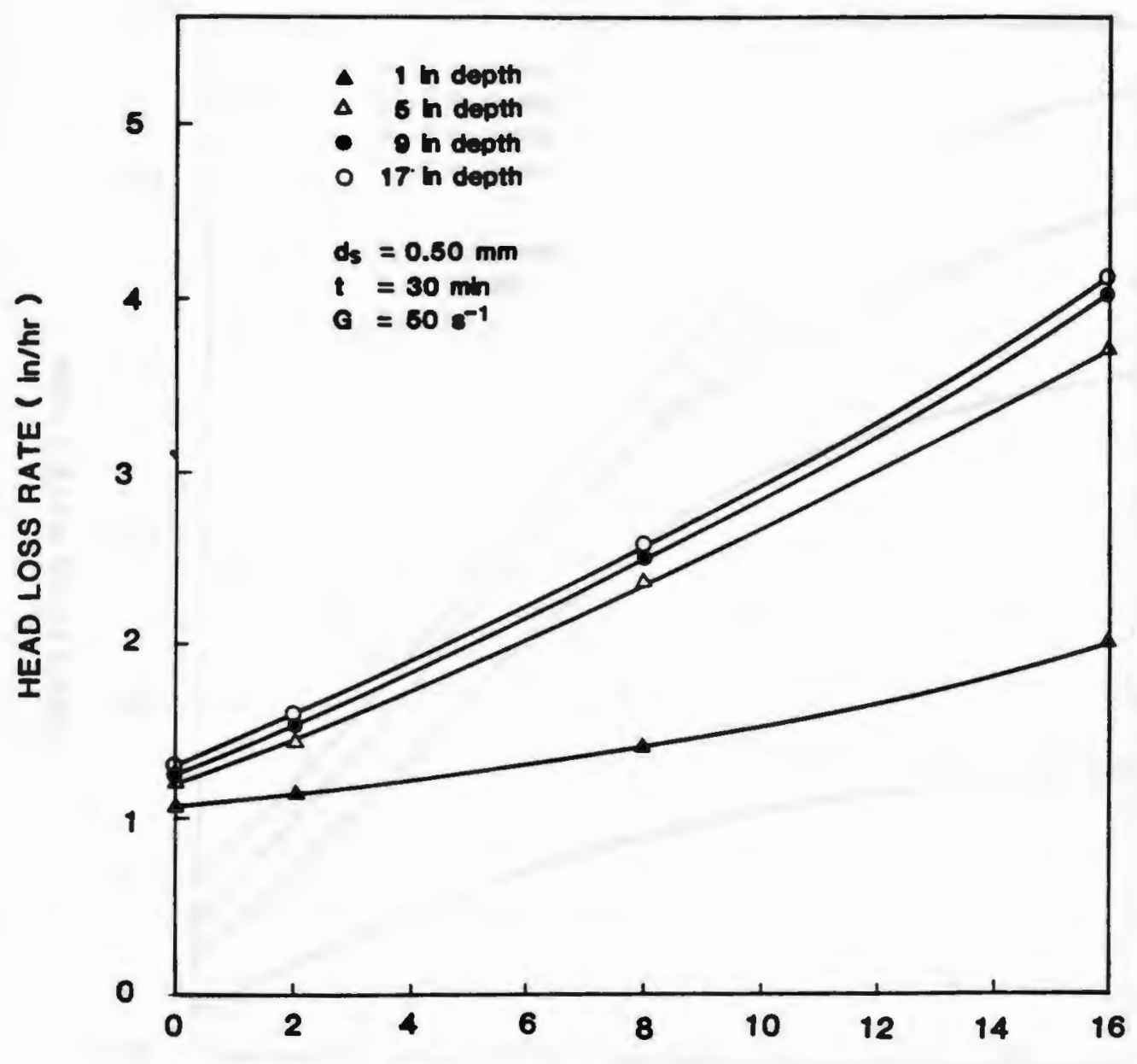

ALUM DOSE ( $\mathrm{mg} / \mathrm{l}$ )

Figure 4.46 Rate of head loss development versus alum dose for medium sand at various sand depths. 


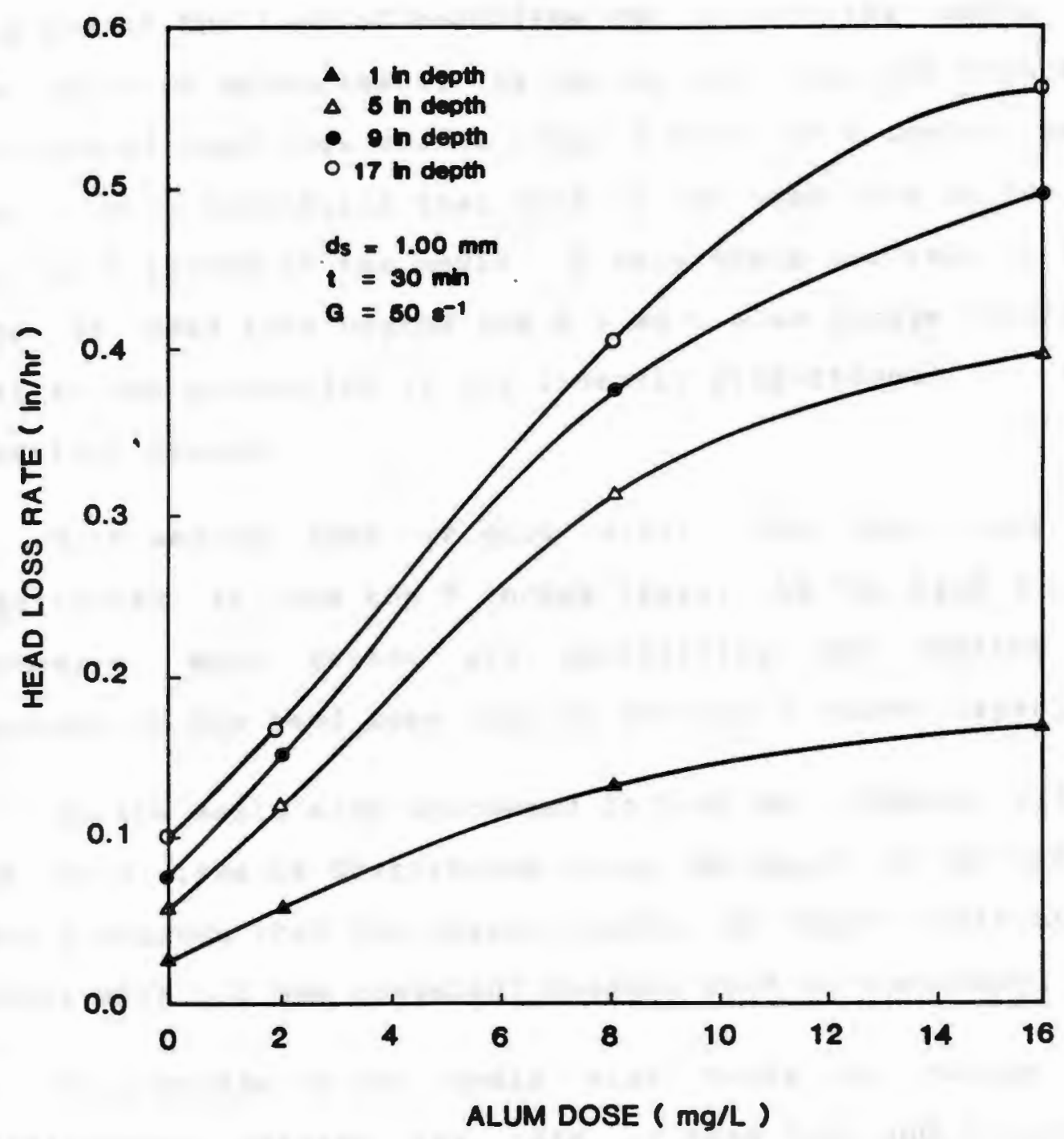

Figure 4.47 Rate of head loss development versus alum dose for coarse sand at various sand depths. 
is caused by the top one inch layer for all coagulant dosages. In the figures, each line represents the head loss rate due to the loss of head from the top of the media to the point of measurement. As can be seen from the figures, the rate of head loss curves after a depth of 5 inches were very tight indicating that most of the head loss is due to the top 5 inches of the media. A very sharp increase in the rate of head loss beyond the $8.0 \mathrm{mg} / \mathrm{L}$ alum dosage suggests that solids production is not linearly proportional to the coagulant 'dosage.

With medium sand (Figure 4.46), the head loss is distributed in the top 5 inches layer. As the alum dosage increases, more solids are penetrating and causing an increase in the head loss only in the top 5 inches layer.

As the media size increased to $1.00 \mathrm{~mm}$ (Figure 4.47), the head loss is distributed along the depth of the media. This indicates that for coarse media in depth filtration occurs with all the coagulant dosages used in the study.

An increase in the media size tends to change the relationship between the rate of head loss and coagulant dose. With fine media, a strong exponential relationship exists between the rate of head loss and alum dose. With coarse media, the rate of head loss tends to level off at the $16.0 \mathrm{mg} / \mathrm{L}$ alum dose (compare Figures 4.45 and 4.47 ). This is because for all of the dosages used, the particles were deposited within the sand bed (in depth filtration) for 
the coarse media filter. For the fine media filter, the higher the coagulant dosage the higher the rate of head loss at the top layer of the media.

The best fit for the curves in Figures 4.45 and 4.46 was found to be an exponential type. The coefficient of determination is 0.97 and above (Table 4.14). For the coarse media (Figure 4.47 ), the best fit was found to be a linear regression equation with a coefficient of determination of 0.93 and above (Table 4.14 ).

\subsubsection{Influence of wixing Intensity}

Plots of the total head loss against run time for three different mixing intensities are shown in Figures $D-4, D-5$, and D-6 (Appendix D) for filters number 1, 2, and 3 , respectively. In all the three figures, an increase in the mixing intensity from 25 to $100 \mathrm{~s}^{-1}$ brought about a decrease in the rate of head loss. However, with fine media there exists a significant difference between the influence of low and high mixing intensities. The coarse media showed less differences due to the size of pores within the media.

The best fit for the curves was also found to be a linear equation with a high coefficient of determination values (Tables $4.11,4.12$, and 4.13 ). The rate of the head loss for different depths is plotted in Figures $4.48,4.49$, and 4.50 and listed in Tables E-1, E-2, and E-3 (Appendix E) . 
Table 4.14- Coefficients of Determination for the Relationships between the Coagulant Dosage, Mixing Intensity, Mixing Time and the Rate of Head Loss for the three Sand Filters

\begin{tabular}{|c|c|c|c|c|c|c|}
\hline $\begin{array}{l}\text { Independent } \\
\text { Variable }\end{array}$ & $\begin{array}{l}\text { Filter } \\
\text { No. }\end{array}$ & 1 & $\begin{array}{l}\text { Sand } \\
5\end{array}$ & $\underset{9}{\text { Depth }}$ & $\begin{array}{c}\text { nches ) } \\
13\end{array}$ & 17 \\
\hline Coagulant & 1 * & 0.9736 & 0.9862 & 0.9860 & 0.9859 & 0.9855 \\
\hline \multirow{2}{*}{ Dosage } & $2 *$ & 0.9934 & 0.9890 & 0.9876 & 0.9868 & 0.9859 \\
\hline & $3 * *$ & 0.9341 & 0.9403 & 0.9518 & 0.9592 & 0.9702 \\
\hline \multirow{3}{*}{$\begin{array}{l}\text { Mixing } \\
\text { Intensity }\end{array}$} & $1 *$ & 0.8591 & 0.9584 & 0.9807 & 0.9688 & 0.9730 \\
\hline & 2 * & 0.9988 & 0.9793 & 0.9783 & 0.9791 & 0.9807 \\
\hline & $3 * *$ & 0.8132 & 0.9368 & 0.8082 & 0.9215 & 0.8473 \\
\hline \multirow{3}{*}{$\begin{array}{l}\text { Mixing } \\
\text { Time }\end{array}$} & $1 *$ & 0.9390 & 0.9586 & 0.9590 & 0.9592 & 0.9598 \\
\hline & 2 * & 0.9297 & 0.9596 & 0.9635 & 0.9645 & 0.9641 \\
\hline & $3 * *$ & 0.9086 & 0.8714 & 0.8604 & 0.8709 & 0.8692 \\
\hline
\end{tabular}

* Exponential relationship

* Linear relationship 


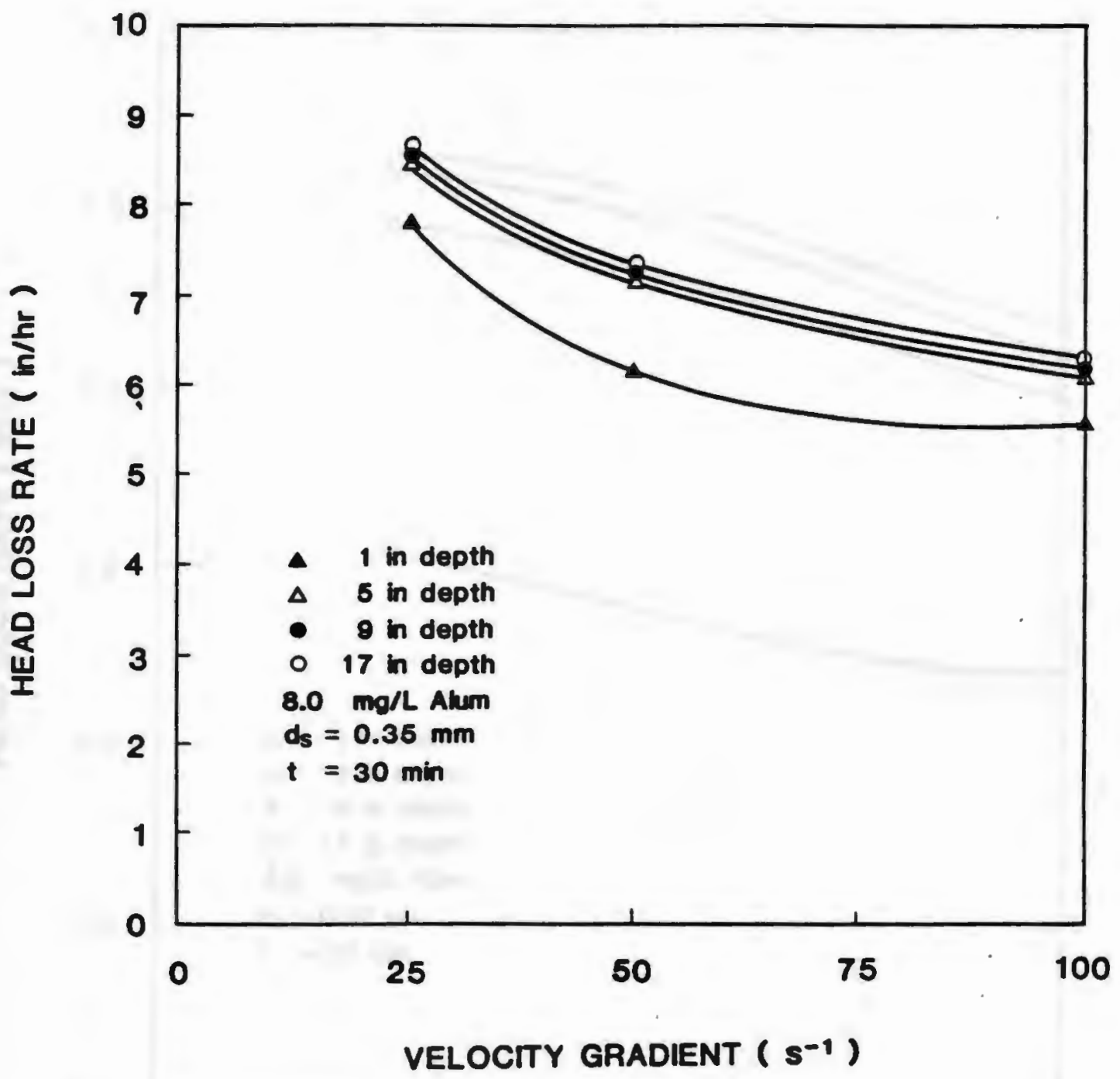

Figure 4.48 Rate of head loss development versus mixing intensity for fine sand at various sand depths. 


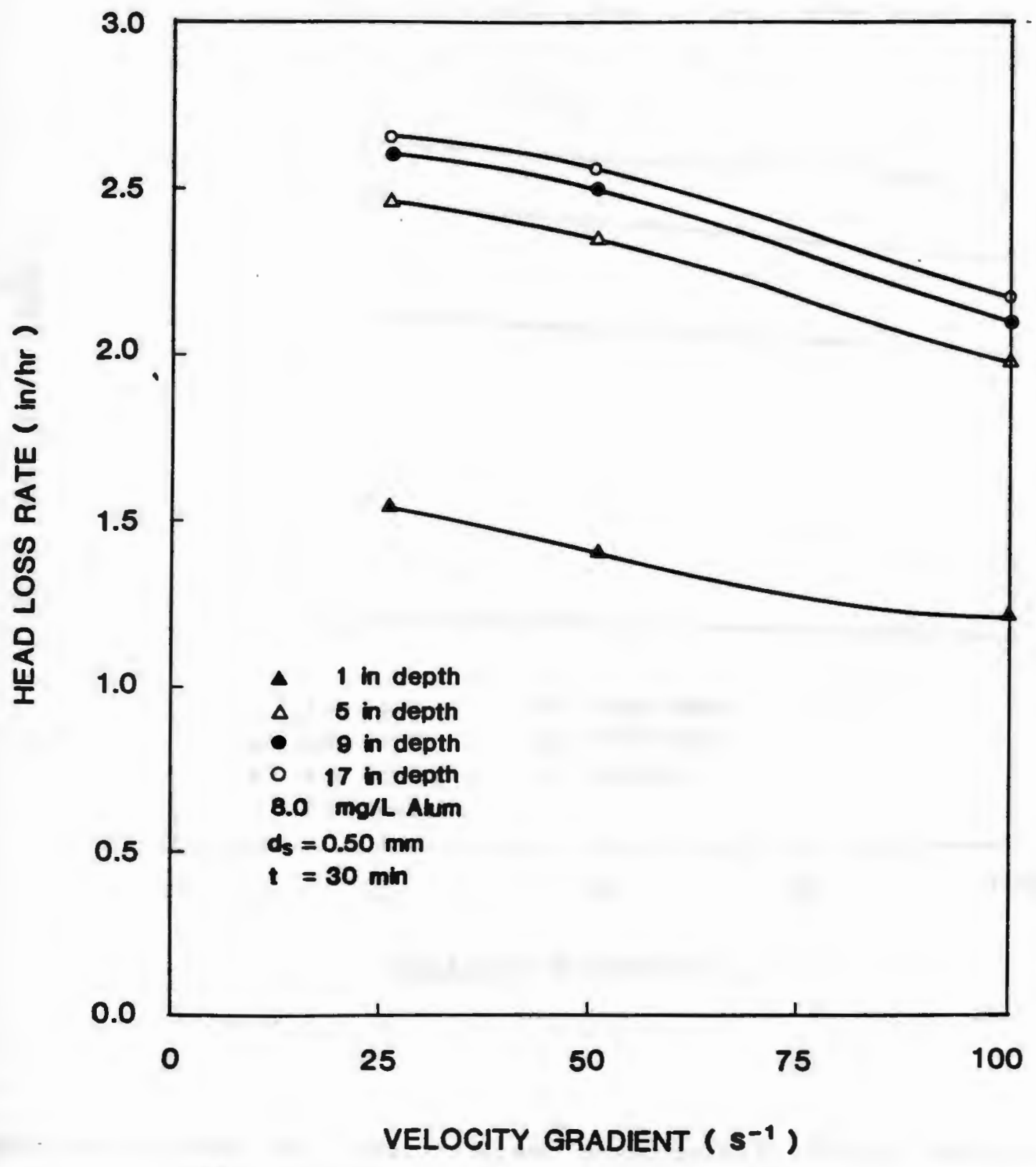

Figure 4.49 Rate of head loss development versus mixing intensity for medium sand at various sand depths. 


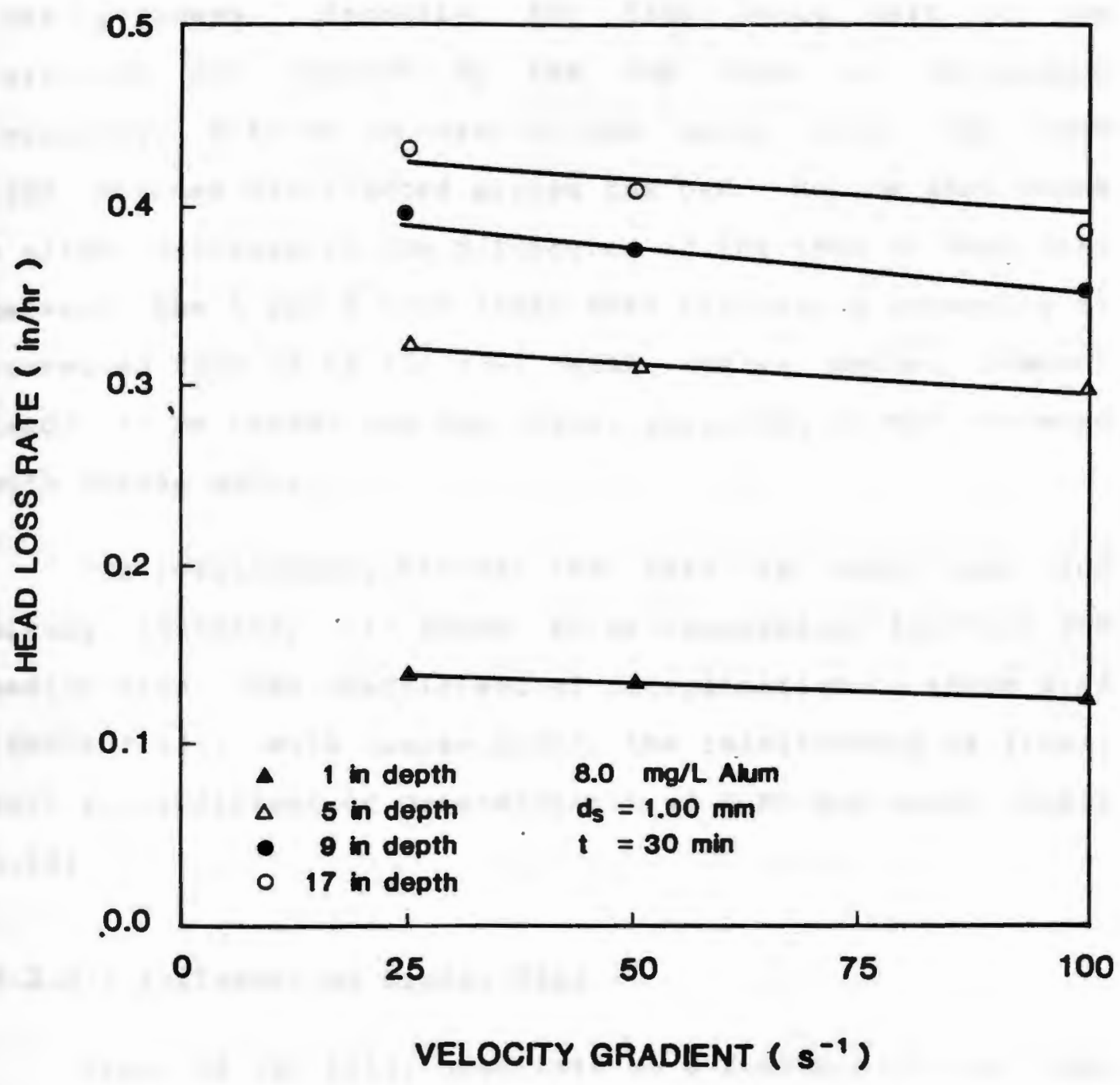

Figure 4.50 Rate of head loss development versus mixing intensity for coarse sand at various sand depths. 
The first observation is that the rate of head loss becomes less dependent on the mixing intensity as the media size increases. Secondly, for fine media most of the particles are removed by the top layer at low mixing intensity. With an increase in the media size, the head loss becomes distributed across the bed. Medium sand shows a slight decrease in the difference in the rate of head loss between the 1 and 5 inch layer when the mixing intensity is increased from 25 to $100 \mathrm{~s}^{-1}$. With coarse media, removal tends to be inside the bed, hence straining is not achieved with coarse media.

The relationship between the rate of head loss and mixing intensity is found to be exponential for fine and medium sand. The coefficient of determination is above 0.85 (Table 4.14). With coarse media, the relationship is linear with a coefficient of determination of 0.80 and above (Table 4.14).

\subsubsection{Influence of Mixing Tine}

Plots of the total head loss as a function of run time for three different mixing times are shown in Figures D-7, D-8, and D-9 (Appendix D) for filter number 1, 2, and 3, respectively. In all of the three figures, the 15 minutes mixing time brought the highest head loss development. This is because the 15 minutes gave the largest floc formation as mentioned earlier. With the increase in the mixing time, a decrease in the rate of head loss is observed. However, the 
decrease is not linearly proportional to the mixing time.

As the media size increases, the influence of high mixing time becomes less significant in terms of the head loss. This can be seen by comparing Figures $D-7$ and $D-9$. In Figure $D-7$ there is a significant difference in the rate of head loss between mixing times of 30 and 45 minutes $(2.28$ in/hr based on the total depth) whereas in Figure D-9 the difference is not very significant $10.05 \mathrm{in} / \mathrm{hr}$ based on the total depth).

Again the curves in Figures $D-7, D-8$, and $D-9$ were best fit to a linear regression equation. The slope of the lines is the rate of head loss and these are plotted in Figures 4.51, 4.52, and 4.53 and listed in Tables E-1, E-2, and E-3 (Appendix E).

The first observation from the three figures is that with all mixing times, the removal of particles with fine media is accomplished by surface straining. With medium sand, more solids are deposited in the one to five inch layer from the top of the sand bed and with coarse media, the head loss is distributed in descending manner with depth. Secondly, as the mixing time increases, the difference in the rate of head loss between the first and second layer decreases with all three sizes of media. In Figure 4.51, the difference in the rate of head loss between the 1 and 5 inch depth at times 15 and 45 minutes is 0.79 and $0.59 \mathrm{in} / \mathrm{hr}$, respectively. For Figure 4.52 these values 


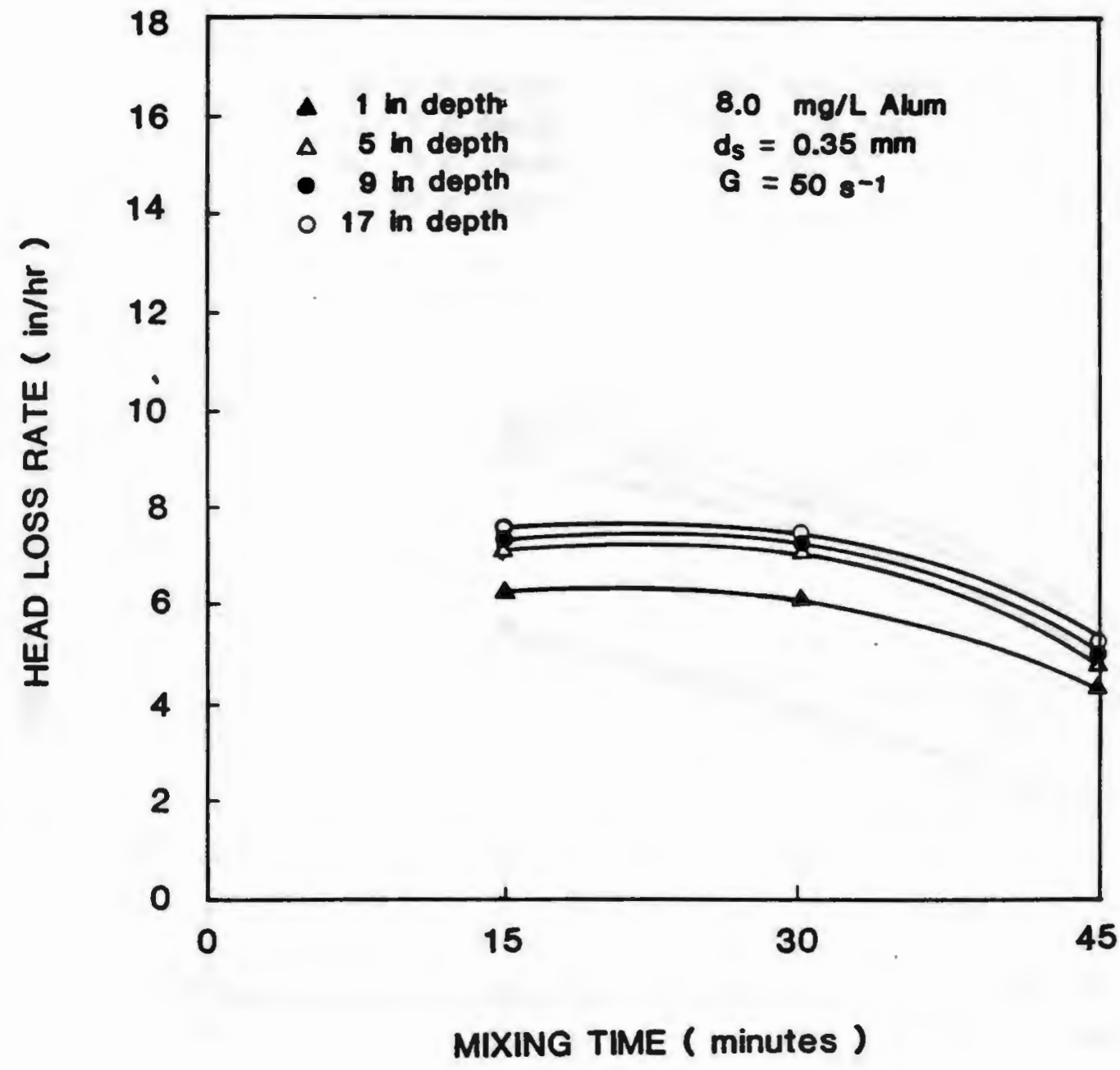

Figure 4.51 Rate of head loss development versus mixing time for fine sand at various sand depths. 


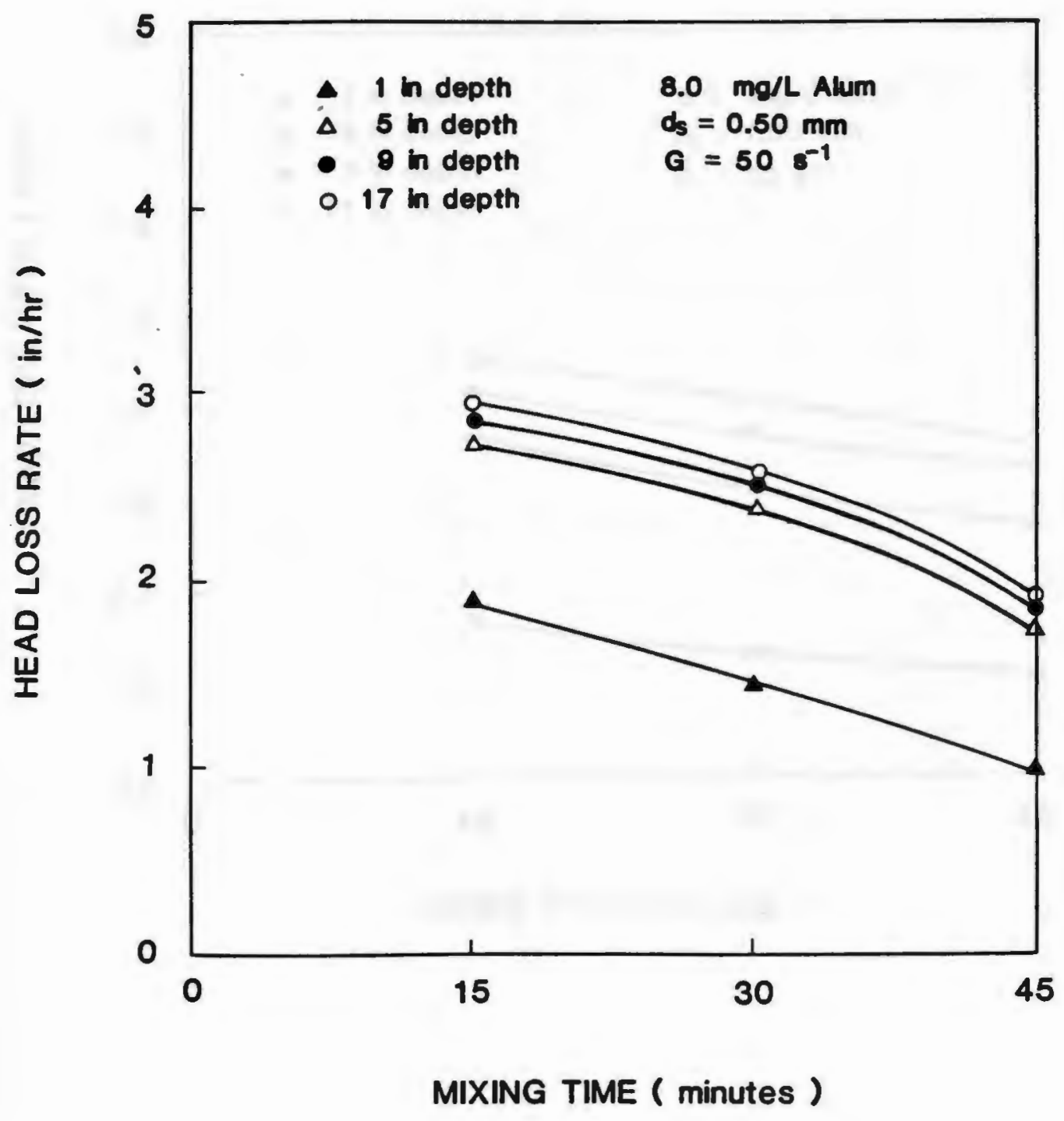

Figure 4.52 Rate of head loss development versus mixing time for medium sand at various sand depths. 


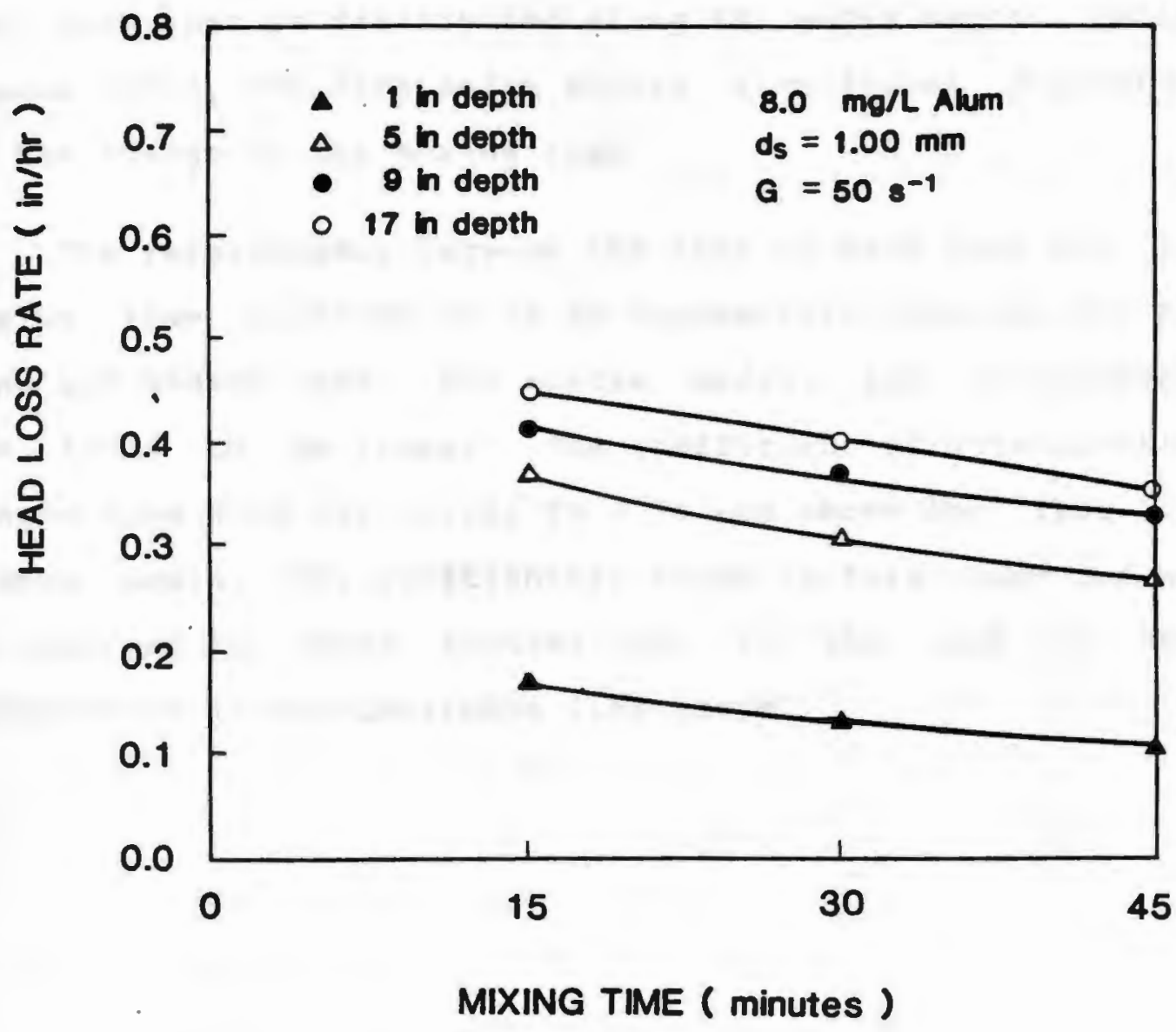

Figure 4.53 Rate of head loss development versus mixing time for coarse sand at various sand depths. 
are 0.81 and $0.73 \mathrm{in} / \mathrm{hr}$ whereas for Figure 4.53 they are 0.20 and $0.17 \mathrm{in} / \mathrm{hr}$. This indicates that an increase in mixing time causes deeper particle penetration, therefore the head loss is distributed along the media depth. Unlike coarse media, the fine media showed significant dependence on the change in the mixing time.

The relationship between the rate of head loss and the mixing time is found to be an exponential function for the fine and medium sand. For coarse media, the relationship was found to be linear. The coefficient of determination ranged from 0.99 for coarse to 0.74 and above for fine and medium media. The relationship found in this study can not be compared to other studies due to the lack of such information in the available literature. 


\section{CONCLUSIONS}

Based on the results obtained from this study, the following conclusions can be drawn:

1. $\beta$, the slope of the particle size distribution curve, can be used as an optimization parameter for coagulant dosage, mixing intensity and mixing time for low suspended solids content waters having a pH between 6 and 9 .

2. Up until the optimum flocculation time, the change in $\beta$ as well as the remaining particles can be modeled by a first order reaction equation, however, in order to model flocculation up to a point beyond the optimum mixing time, a second order polynomial is required.

3. As the coagulant dosage increased, the distribution slope, $\beta$, decreased. The coagulant dosage was the most influential on the filter effluent quality. The distribution slope increases with the increase in the mixing intensity or time (physical variables). These physical variables have less influence on the effluent quality as compared to the coagulant dosage.

4. As the influent distribution slope decreases, the rate of head loss development increases due to the formation of larger flocs which have less tendancy to deposit within the sand bed.

5. The effluent quality and particle size distribution 
from the filters packed with smaller diameter filter media proved to be more sensitive to changes in the influent particle size distribution than did filters with coarser media.

6. The relationship between the rate of head loss development and the influent particle size distribution was found to be an exponential function for both fine and medium filter media and linear for coarse media. 


\section{RECOMMENDATIONS}

Further research is needed in the area of particle size distribution as related to water treatment practices. More specific recommendations for future research in this area includes the following:

1. Investigation of the change of the particle size distribution when dealing with waters that have high concentrations of suspended particulate matter. This includes the investigation of the influence of sedimentation prior to granular media filtration on the particle size distribution.

2. The influence of rapid mixing on the particle size distribution and the influence it has on granular media filtration in the contact flocculation treatment process.

3. The influence of graded, dual and multi media filtration on the effluent particle size distribution with relation to the influent particle size distribution.

4. Selection of an optimum granular media gradation based on the influent suspended solids concentration. 


\section{REFERENCES}

1. Adin, A. and Rebhun, M. (1974). "High-Rate Contact Flocculation-Filtration with Cationic Polyelectrolytes. J. AWWA, 66(2), 109-117.

2. American Water Works Association (1968). "Quality Goals for Potable Water." J. AWWA, 60(12), 1317-1322.

3. Amirtharajah, A. (1978). "Design of Rapid Mix Units." In Water Treatment Plant Design., Sanks, R.L., Ed., Ann Arbor Science, Ann Arbor, Michigan. 131-148.

4. Amirtharajah, A. and Mills, K.M. (1982). "Rapid-Mix Design for Mechanisms of Alum Coagulation." J. AWWA, $74(4), 210-216$.

5. Amirtharajah, A. and Trusler, S.L. (1986). "Destabilization of Particles by Turbulent Rapid Mixing." J. Env. Eng. Div. ASCE, 112(EE6), 1085-1108.

6. Argaman, Y. and Kaufman, W.J. (1970). "Turbulence and Flocculation." J. San. Eng. Div. ASCE, 96(SA2), 223-241

7. Argaman, Y.(1971). "Pilot Plant studies of Flocculation" J. AWWA, 63(12), 775-777.

8. Beard II, J.D. and Tanaka, T.S. (1977). "A Comparison of Particle Counting and Nephelometry." J.AWWA, 69(10) , 533-538.

9. Benefield, L.D.; Judkins, J.F.; and Weand, B.L. (1982). "Process Chemistry for Water and wastewater Treatment" Prentice-Hall, Inc., Englewood Cliffs, N.J.

10. Bernardo, L.D., and Cleasby, J.L. (1980). "DecliningRate Versus Constant Rate Filtration." J. Env. Eng. Div. ASCE, 106 (EE4), 1023-1041.

11. Bitton, G. and Mitchell, R. (1974a)." Effects of Colloids on the survival of Bacteriophages in sea water." water Research, 8(4), 227-229.

12. Bitton, G. and Mitchell, R. (1974b). "Protection of E. Coli by Montmorillonite in Seawater." J. Env. Eng. Div. ASCE, 100 (EE6), 1310-1313.

13. Black, A.P.; Birkner, F.B. and Morgan, J.J. (1965a). " Destabilization of Dilute clay suspensions with Labeled Polymers." J. AWWA, 57(12), 1547-1560.

14. Black, A.P. and Hannah, S.A. (1965b). " Measurement of Low Turbidities." J. AWWA, 57(7), 901-916. 
15. Boadway, J.D. (1978). " Dynamics of Growth and Breakage of Alum Floc in Presence of Fluid Shear." J. Env. Eng. Div. ASCE, 104(EE5), 901-915.

16. Camp, G.M. (1955). "Flocculation and Flocculation Basins." J. San. Eng. Div. ASCE, 120(SA1), 1-16.

17. Camp, T.R. (1968). "Floc Volume Concentration." J. AWWA, $60(6), 656-673$.

18. Cleasby, J.L. and Baumann, E.R. (1962). " selection of Sand Filtration Rates." J. AWWA, 54(5), 579-602.

19. Cleasby, J.L., Williamson, M.M., and Bauman, E.R.(1963) "Effect of Filtration Rate changes on Quality." $\mathrm{J}$. AWWA, 55(7), 869-877.

20. Cleasby, J.L.(1969a). "Filter Rate Control without Rate Controliers." J. AWWA, 61(4), 181-185.

21. Cleasby, J.L. (1969b). "Approaches to a Filtrability Index for Granular Filters." J. AWWA, 61(8), 372-381.

22. Cleasby, J.L. and Bernardo, L.D. (1980). "Hydraulic Considerations in Declining-Rate Filtration." J. Env. Eng. Div. ASCE, $106($ EE6), 1043-1055.

23. Cleasby, J.L. (1984). "Is Velocity Gradient a Valid Turbulent Flocculation Parameter ? "J. Env. Eng. Div. ASCE, 110(EE5), 875-898.

24. Committee Report (1971)." state of the Art of Coagulation." J. AWWA, 63(2), 99-108.

25. Committee Report $(1980)$." The status of Direct Filtration." J. AWWA, 72(7), 405-411.

26. Collins, M.R., Amy, G.L. and Bryant, C.W. (1987). "Evaluation of Factors Affecting Performance of Direct Filtration." J. Env. Eng. Div. ASCE, 113(EE2), 330-344.

27. Cookson, J.T., Jr. (1970). " Removal of Submicron Particles in Packed Beds." J. Environmental Science and Technology, 4(2), 128-134.

28. Cornwell, D.A., and Bishop, M.M. (1983). " Determining velocity Gradients in Laboratory and Full-scale Systems." J. AWWA, 75(9), 470-475.

29. Culp,R.L. (1977). "Direct Filtration." J. AWWA, 69(7), 375-378.

30. Dempsey, B.A.; Ganho, R.M. and O'Melia, C.R. (1984a). "The Coagulation of Humic Substances by Means of 
Aluminum Salts." J. AWWA, 76(4), 141-150.

31. Dempsey, B.A. and O'Melia, C.R. (1984b). "Removal of Naturaliy Occurring Compounds by Coagulation and sedimentation." Critical Reviews in Environmental Control, 14(4), 311-330.

32. Edwards, G.A., and Amirtharajah, A. (1985). "Removing Color Caused by Humic Acids." J. AwWA, 77(3), 50-57.

33. Fair, G.M. and Gemme11, R.S. (1964). "A Mathematical Model of Coagulation." J. Colloid Science, 19(4), 360-372.

34. Foley, P.D. (1980). "Experience with Direct Filtration at ontario's Lake Huron Treatment Plant." J. AWWA, $72(3), 162-164$.

35. Ghosh, M.M., Jordan, T.A., and Porter, R.L. (1975). " Physicochemical Approach to water and Wastewater Filtration." J. Env. Eng. Div. ASCE, 101(EE1), 71-86.

36. Habibian, M.T., and O'Melia, C.R. (1975). "Particles, Polymers, and Performance in Filtration." J. Env. Eng. Div. ASCE, 101(EE4), 567-583.

37. Hannah, S.A.: Cohen, J.M. and Robeck, G.G. (1967). "Measurement of Floc strength by Particle Counting." J. AWWA, 59(7), 843-858.

38. Harris, H.S.: Raufman, W.J. and Rrone, R.B. (1966). "Orthokinetic Flocculation in Water Purification." J. San. Eng. Div. ASCE, 92(SA6), 95-111.

39. Huang, J.Y.C. and Garcia-Maura, F. (1986). "Effect of Influent Property on Filter Performance." J. Env. Eng. Div. ASCE, 112(EE4), 701-717.

40. Hudson, H.E.(1965). "Physical Aspects of Flocculation." J. AWWA, 57(7), 885-892.

41. Hudson, H.E., and wolfner, J.P. (1967). "Design of Mixing and flocculating Basins." J. AWWA, 59(10). 1257-1267.

42. Hudson, H.E., Jr. (1969)." Physical Aspects of Filtration." J. AWWA, 61(1), 3-7.

43. Hudson, H.E.(1973). "Evaluation of Plant Operating and Jar-Test Data." J. AWWA, 65(5), 368-375.

44. Hudson, H.E., and Wagner, E.G.(1981). "Conduct and Uses of Jar Tests." J. AWWA, 73(4), 218-223.

45. Hutchinson, C.w. (1985). "On-Line Particle counting 
Improves Filter Efficiency." Water/Engineering and Management, $132(7), 20-25$.

46. Hutchison, W. and Foley, P.D. (1974). "Operational and Experimental Results of Direct Filtration." J. AWWA, $66(2), 79-87$.

47. Hutchison, W.R. (1976). "High-Rate Direct Filtration." J. AWWA, 68(6)292-298.

48. Ives, K.J. and Sholji, I. (1965). "Research on Variables Affecting Filtration." J. San. Eng. Div. ASCE, 91(SA4), $1-18$.

49. Ives, K.J. and Bhole, A.G. (1973)." Theory of Flocculation for Continous Flow Systems." J. Env. Eng. Div. ASCE, 99 (EE1), 17-34.

50. Ives; K.J. (1978a). "A New Concept of Filterability." Progress in water Technology,10(5/6), 123-137.

51. Ives, R.J. (1978b). "Experimental Methods (2) Dilute Suspensions." In The scientific Basis of Flocculation, Ives, R.J., Ed., Sijthoff and Noordhoff Int., Alphen aan den Rijn. The Netherlands, 165-192.

52. Iwasaki, T.(1937). "Some Notes on Sand Filtration." J. AWWA, 29(10), 1591-1602.

53. Jordan, T.A.; Ghosh, M.M. and Boyd, R.H., Jr. (1974). "Physico-Chemical Aspects of Deep-Bed Filtration." J. water Pollution Control Federation, 46(12), 2745-2754.

54. Jung, H. and Savage, E.S. (1974). "Deep-Bed Filtration." J. AWWA, 66(2), 73-78.

55. Kane, J.C.; La Mer, V.K. and Linford, H.D. (1963). "The Filtration of Silica Dispersions Flocculated by High Polymers." J. Physical Chemistry, 67(10), 1977-1981.

56. Kavanaugh, M.C., Tate, C.H., Trussell, A.R., Trussell, R.R. and Treweek, G. (1980). "Use of Particle Size Distribution Measurements for selection and Control of Solid/Liquid separation Processes." In Particulates in water: Characterization, Fate, Effects, and Removal, Kavanaugh, M.C. and Leckie, J.O. Editors, American Chemical Society, washington, D.C., 305-328.

57. Kawamura, S. (1976). " Considerations on Improving Flocculation." J. AWWA, 68(6), 328-336.

58. Kirchman, W.B. and Jones, W.H. (1972). "High Rate Filtration." J. AWWA, 64(3), 157-161.

59. Lagvankar, A.L., and Gemme11, R.S. (1968). "A Size- 
Density Relationship for Flocs." J. AWWA, 60(9), 1040-1046.

60. Lai, R.J.: Hudson, H.E., and Singley, J.E. (1975). "Velocity Gradient Calibration of Jar-Test Equipment." J. AWWA, 67(10),553-557.

61. Lawler, D.F.. Izurieta, E., and Kao, C.(1983). " Change in Particle size Distributions in Batch Flocculation." J. AWWA, 75(12), 604-612.

62. Leclerc, A.F. and Tri Vu, T. (1972). "Desorption and Retentivity of Filtering Materials." J. San. Eng. Div. ASCE, $98($ SÁ 6$), 937-949$.

63. Letterman, R.D.: Quon, J.E. and Gemmell, R.S. (1973). "Influence of Rapid-Mix Parameters on Flocculation." J. AWWA, 65(11), 716-722.

64. Letterman, R.D., Sama, R.R. and Didomenico, E.S.(1979). "Direct Filtration Using Polyelectrolyte Coagulants." J. AWWA, $71(6), 332-338$.

65. Loganathan, P. and Maier, W.J. (1975). "Some surface Chemical Aspects in Turbidity Removal by sand Filtration." J. AWWA, 75(6), 336-342.

66. Logsdon, G.S. (1978). "Direct Filtration- Past, Present, Future." Civil Engineering, ASCE, 48(7), 68-73.

67. Mackrle, v. and Mackrle, s. (1961)." Adhesion in Filters." J. San. Eng. Div. ASCE, 87(SA5), 17-32.

68. Mackrle, S. (1962). "Mechanism of Coagulation in water Treatment." J. San. Eng. Div. ASCE, 88(SA3), 1-13.

69. Matsuo, T. and Unno, H. (1981). "Forces Acting on Floc and strength of Floc." J. Env. Eng. Div. ASCE, $107($ EE3), 527-545.

70. McCormick, R.F. and King, P.H. (1982). "Factors that Affect Use of Direct Filtration in Treating surface Waters." J. AWWA, 74(5), 234-242.

71. McCoy, W.F. and Olson, B.H.(1986). "Relationship Among Turbidity, Particle Counts and Bacteriological Quality Within Water Distribution Lines." Water Research. $20(8)$, 1023-1029.

72. Monscvitz, J.J.. Rexing, D.J., Williams, R.G. and Heckler, J.(1978). "Some Practical Experience in Direct Filtration." J. AWWA, 70(10), 584-588.

73. Monscuitz, J.J. and Rexing, D.J. (1983). "Direct Filtration control by particle counting." Proc. Ann. 
AWWA Conf., 1169-1184.

74. O'Connell, R.T. (1978). " Suspended Solids Removal." In Water Treatment Plant Design, Sanks, R.L. Ed. Ann Arbor Science Publishers, Ann Arbor, Mich.,283-298.

75. O'Melia, C.R. and Crapps, D.K. (1964). "Some Chemical Aspects of Rapid Sand Filtration." J. AWWA, 56(10), 1326-1344.

76. O'Melia, C.R. (1965). "Discussion of Theory of water Filtration." J. San. Eng. Div. ASCE, 91(SA2), 92-98.

77. O'Melia, C.R. and Stumm, พ. (1967a). " Aggregation of silica Dispersions by Iron (III)." J. Colloid and Interface Science, $23(3), 437-447$.

78. O'Melia, C.R. and Stumm, w. (1967b). "Theory of water Filtration." J. AWWA, 59(11), 1393-1412.

79. O'Melia, C.R. (1969). "A Review of the Coagulation Process." Public Works, 100(5), 87-98.

80. O'Melia, C.R. (1972). "Coagulation and Flocculation." In Physiochemical Processes for water Quality Control, Weber, W.J., Jr., Ed., John Wiley and Sons, New York, N.Y., 61-109.

81. O'Melia, C.R. (1978a). " Coagulation in wastewater Treatment." In The Scientific Basis of Flocculation, Ives, R.J., Ed., Sijthoff and Noordhoff Int., Alphen aan den Rijn, The Netherlands, 219-268.

82. O'Melia, C.R. and $A l i, W .(1978 b)$. "The Role of Retained Particles in Deep Bed Filtration." Progress in Water Technology, 10(5/6), 167-182.

83. O'Melia, C.R. (1978c). "Coagulation." In water Treatment Plant Design for the Practicing Engineer, Sanks, R.L., Ed.. Ann Arbor Science, Ann Arbor, Mich., 65-82.

84. O'Melia, C.R. (1980). "Aquasols: the Behavior of Small Particles in Aquatic Systems." J. Environmental Science and Technology, 14(9), 1052-1060.

85. O'Melia, C.R. (1985). "Particles, Pretreatment, and Performance in water Filtration." J. Env. Eng. Div. ASCE, 111(EE6), 874-889.

86. Ott, C.R. and Bogan, R.H. (1970). "Theoretical Evaluation of Filter Modeling Experiments." J. San. Eng. Div. ASCE, 96(SA2), 455-465.

87. Packham, R.F. (1965). " Some studies of the Coagulation of Dispersed Clays with Hydrolyzing Salts." J. Colloid 
science, 20(1), 81-92.

88. Parker, D.S.; Kaufman, W.J. and Jenkins, D. (1972). "Floc Breakup in Turbulent Flocculation Processes." J. San. Eng. Div. ASCE, 98(SAl), 79-99.

89. Ramaley, B.L.; Lawler, D.F.; Wright, W.C. and O'Melia, C.R. (1981)." Integral Analysis of water Plant Performance." J. Env. Eng. Div. ASCE, 107(EE3),547-562.

90. Reed, G.D., and Mery, P.C. (1986). "Influence of Floc Size Distribution on Clarification." J. AWWA, 78(8), $75-80$.

91. Sakthivadival, R.: Thanikachalam, V. and Seetharaman, S. (1972). "Head-Loss Theories in Filtration." J. AWWA, $64(4), 233-238$.

92. Shea,,T.G.: Gates, W.E. and Argaman, Y.A. (1971). " Experimental Evaluation of operating variables in contact Flocculation." J. AWWA, 63(1), 41-48.

93. Shinnar, R. (1961)." On the Behaviour of Liquid Dispersions in Mixing Vessels." J. Fluid Mechanics, $10(2), 259-275$.

94. Shull, K.E. (1967). "Filtrability Techniques for Improving Water Clarification." J. AWWA, 59(9), 1164-117i.

95. Silverman, G.S.; Nagy, L.A. and Olson, B.H. (1983). "Variations in Particulate Matter, Algae, and Bacteria in an Uncovered, Finished-Drinking Water Reservoir." J. AWWA, 75(4), 191-195.

96. Smith, C.V.(1967). " Determination of Filter Media zeta Potential." J. San. Eng. Div. ASCE, 93(SA5), 91-107.

97. Spielman, L.A. and Goren, S.L. (1970). "Capture of Small Particles by London Forces from Low-Speed Liquid Flows" $\mathrm{J}$. Environmental Science and Technology, 4(2), 135-140.

98. Stumm, W., and O'Melia, C.R. (1968). "stoichiometry of Coagulation." J. AWWA, 60(5), 514-539.

99. Stumm, W. (1977). "Chemical Interaction in Partial Separation." J. Environmental Science and Technology, $11(11)$, 1066-1070.

100. Sweeney, G.E. and Prendiville, P.W. (1974). " Direct Filtration: An Answer to a City's water Needs." $\mathrm{J}$. AWWA, 66(2), 65-71.

101. Tanaka, T.S., and Pirbazari, M. (1986). "Effects of Cationic Polyelectrolytes on the Removal of suspended 
Particulates During Direct Filtration." J. AWWA, 78(12), 57-65.

102. Tate, C.H.: Lang, J.S., and Hutchinson, H.L. (1977). "Pilot plant Tests of Direct Filtration." J. AWWA, $69(7), 379-384$.

103. Tate, C.H. and Trussell, R.R. (1978). "The Use of Particle Counting in Developing Plant Design Criteria." J. AWWA, 70(12), 691-698.

104. TeKippe, R.J.., and Ham, R.K. (1970). " Coagulation Testing: A Comparison of Techniques-- Part I." J. AWWA, $62(9), 594-602$.

105. TeKippe, R.J., and Ham, R.K. (1970). " Coagulation Testing: A Comparison of Techniques-- Part II."J. AWWA, $62(10), 620-628$.

106. TeRippe, R.J., and Ham, R.K.(1971). "Velocity-Gradient Paths in Coagulation." J. AWWA, 63(7), 439-448.

107. Toregas, G. (1983). " Using Backwashing Kinetics to Evaluate Attachment Mechanisms and Forces During Eiltration." J. AWWA, 75(5), 254-260.

108. Treweek, G.P. and Morgan, J.J. (1977). " size Distributions of Flocculated Particles: Application of Electronic Particle Counters." J. Environmental science and Technology, 11(7), 707-714.

109. Treweek, G.P.(1979). "Optimization of Flocculation Time Prior to Direct Filtration." J. AWWA, 71(2), 96-101.

110. Treweek, G.P. and Morgan, J.J. (1980). "Prediction of Suspension Turbidities from Aggregate size Distribution ." In Particulates in water: Characterization, Fate, Effects, and Removal, Ravanaugh, M.C. and Leckie, J.O. Editors, American Chemical Society, washington, D.C., 329-351.

111. U.S. Environmental Protection Agency (1975). "National Interim Primary Drinking Water Regulations." Federal Register, 40:59566.

112. Vigneswaran, S. and Aim, R.B. (1985). "The Influence of Suspended Particle size Distribution in Deep-Bed Filtration." J. AICHE, 31(2), 321-324.

113. Vrale, L. and Jordan, R.M. (1971)." Rapid Mixing in Water Treatment." J. AWWA, 63(1), 52-58.

114. Wagner, E.G. and Hudson, H.E., Jr. (1982). "Low-Dosage High-Rate Direct Filtration." J. AWWA, 74(5), 256-261. 
115. Wiesner, M.R.; O'Melia, C.R. and Cohen, J.L. (1987). "Optimal Water Treatment Plant Design." J. Env. Eng. Div. ASCE, 113(EE3), 567-584.

116. Westerhoff, G.P.; Hess, A.F. and Barnes, M.J. (1980). "Plant-Scale Comparison of Direct Filtration Versus Conventional Treatment of a Lake Erie Water." J. AWWA, $72(3), 148-155$.

117. Yao, K., Habibian, M.T. and O'Melia, C.R.(1971). "Water and wastewater Filtration: Concepts and Applications." J. Environmental science and Technology 5(11), 1105-1112.

118. Yeh, H., and Ghosh, M.M.(1981). "Selecting Polymers for Direct Filtration." J. AWWA, 73(4), 211-218. 
APPENDIX A

List of the Computer Program 


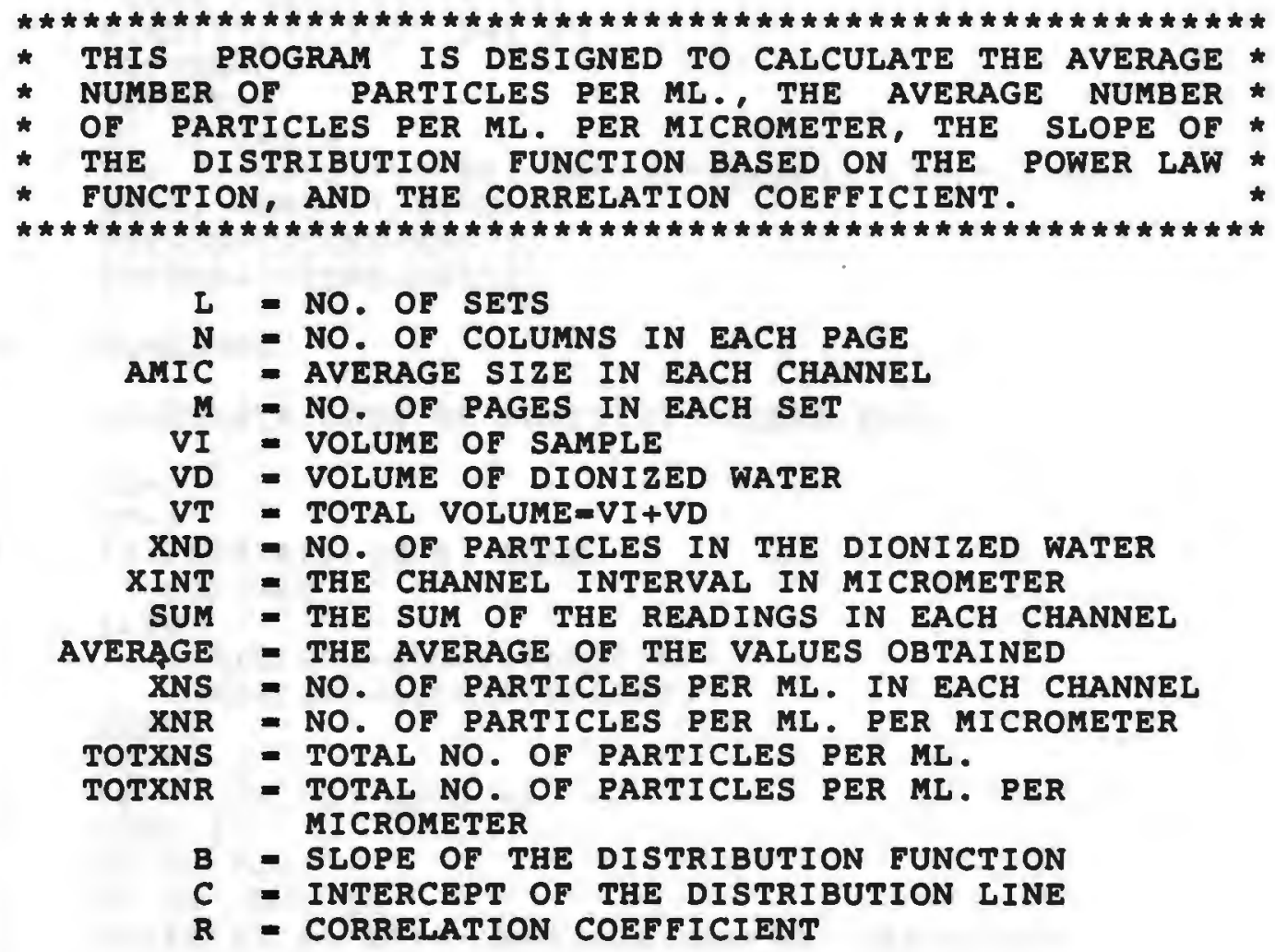

DIMENSION SUM $(1000), \operatorname{AVG}(1000), X N S(1000)$

DIMENSION XNR (1000), YAXIS(20), XAXIS(20)

DIMENSION VT( 20$), \operatorname{VD}(20), V I(20), X N D(200)$

DIMENSION M(30), X(1000), AMIC(12),D(12)

DIMENSION $A(5,5), F(5,5), \operatorname{XINT}(1000)$

$\mathrm{L}=$ ?

$\mathrm{N}=12$

$\operatorname{READ}(? ?, *)$ ( $\operatorname{MMIC}($ IH ) , I H= 1,N)

DO $10 \mathrm{KK}=1$, L

$\operatorname{READ}(? ?, *) M(K K)$

$\operatorname{READ}(? ?, *) \operatorname{VI}(\mathrm{KK}), \operatorname{VD}(\mathrm{KK}), \operatorname{VT}(\mathrm{KK})$

$\operatorname{READ}(? ?, *)$ ( XND ( I I ), I I = 1,N)

$\operatorname{READ}(? ?, *)(X I N T(J J), J J=1, N)$

$M M=M(K K)$

DO $15 I=1, M M$

WRITE $(? ?, 20)$

FORMAT(1H1,T19,'X(J)',T32,' NS ',T47,'NR',T59, 


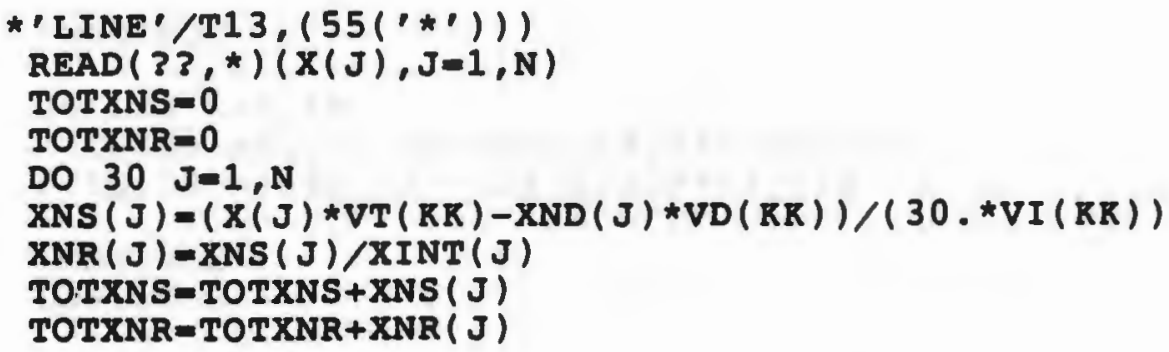

30 CONTINUE

C CALCULATE LOGS OF POSITIVE VALUES ONLY

$I G=0$

$\mathrm{KF}=1$

35 IF (XNR(KF).LE.0) THEN

$$
\begin{aligned}
& \text { I G }=I G+1 \\
& \text { ELSE }
\end{aligned}
$$

$$
\text { XAXIS (KF)-ALOG10 (AMIC (KF)) }
$$

ENDIF

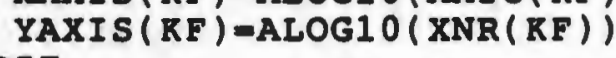

$\mathrm{KF}=\mathrm{KF}+1$

IF(RF.GT.12) GOTO 40

GOTO 35

40 LH=KE-IG-1

DO 50 IO- 1 , LH

WRITE $(? ?, 60) \times(I O), X N S(I O), X N R(I O), Y A X I S(I O)$

60 FORMAT(T15,4E13.5)

50 CONTINUE

C CALCULATE COEFFICIENTS OF EQUATIONS

C INITIALIZE ARRAYS TO 0

DO $70 \quad K M=1,5$

DO $80 \mathrm{KL}=1,5$

$A(K M, K L)=0.0$

$F(K M, K L)=0.0$

80 CONTINUE

70 CONTINUE

DO $90 \mathrm{KN}=1,12$

$D(\mathbf{K N})=0$

90 CONTINUE

DO $100 I Q=1,2$

DO $110 I R=1,2$

IF(IQ+IR.GT.2) GOTO 120

$A(I Q, I R)=L H$

GOTO 110 
120 CONTINUE

DO $130 \mathrm{~K}=1, \mathrm{LH}$

$A(I Q, I R)=A(I Q, I R)+\operatorname{XAXIS}(R) * *(I Q+I R-2)$

$F(I Q, I R)=F(I Q, I R)+\operatorname{YAXIS}(R) * *(I Q+I R-2)$

30 CONTINUE

110 CONTINUE

DO $140 \mathrm{~K}=1$, $\mathrm{LH}$

$I F(I Q . G T .1) \quad D(I Q)=D(I Q)+\operatorname{YAXIS}(R) * \operatorname{XAXIS}(R) * *(I Q-1)$

$I F(I Q \cdot L E .1) \quad D(I Q)=D(I Q)+Y A X I S(K)$

140 CONTINUE

100 CONTINUE

$B=(A(1,1) \star D(2)-A(1,2) \star D(1)) /(A(1,1) \star A(2,2)-A(1,2) \star \star 2)$

$C=(D(1)-B * A(1,2)) / A(1,1)$

$E=L H * F(2,2)-D(1) * \star 2$

$\mathrm{DEN}=\mathrm{LH} * \mathrm{~A}(2,2)-\mathrm{A}(1,2) \star \star 2$

$R=B *((D E N / E) \star \star .5)$

WRITE $(? ?, 150)$ TOTXNS, TOTXNR, B, C, R

150 FORMAT ( $/ 2 \mathrm{X}$, ' TOTAL-XNS=', F10.2,/,2X, 'TOTAL-XNR='

*, F10.2, $/ .2 \mathrm{X}, '$ SLOPE=', F10.6, $/, 2 \mathrm{X}$, ' INTERCEPT='

*,F10.6,/.2X,' CORRELATION COEFEICIENT=',F10.4)

15 CONTINUE

10 CONTINUE

STOP

END 


\section{APPENDIX B}

\section{Pretreatment Data}

- Jar Test

- Pilot-Plant mixing Tank 
Table B-1 - Remaining Number of Particles per ml for $\mathrm{G}=40 \mathrm{~s}^{-1}$, and $\mathrm{pH}=7.00$

\begin{tabular}{|c|c|c|c|c|c|c|c|c|c|c|c|c|c|c|c|}
\hline \multirow{2}{*}{$\begin{array}{l}\text { Alum } \\
\text { Dose } \\
\mathrm{mg} / \mathrm{L}\end{array}$} & \multirow[b]{2}{*}{$\begin{array}{l}\text { Time } \\
\text { min. }\end{array}$} & \multicolumn{13}{|c|}{ Average Particle $\$ 120$, Merons } & \multirow[b]{2}{*}{$\begin{array}{c}\text { Distribution } \\
\text { slope, } \beta\end{array}$} \\
\hline & & 3.5 & 7.5 & 12.5 & 17.5 & 22.5 & 27.5 & 32.5 & 37.5 & 45 & 62.5 & 87.5 & 125 & Total & \\
\hline 5.0 & $\begin{array}{r}3.00 \\
12.65 \\
22.50 \\
36.67 \\
51.00\end{array}$ & $\begin{array}{r}10907 \\
2911 \\
2301 \\
2039 \\
1421\end{array}$ & $\begin{array}{r}6651 \\
1766 \\
1393 \\
1254 \\
810\end{array}$ & $\begin{array}{r}1849 \\
830 \\
663 \\
641 \\
384\end{array}$ & $\begin{array}{l}748 \\
587 \\
478 \\
491 \\
296\end{array}$ & $\begin{array}{l}313 \\
431 \\
356 \\
386 \\
267\end{array}$ & $\begin{array}{l}106 \\
272 \\
236 \\
266 \\
200\end{array}$ & $\begin{array}{r}44 \\
192 \\
189 \\
201 \\
175\end{array}$ & $\begin{array}{r}14 \\
90 \\
92 \\
100 \\
91\end{array}$ & $\begin{array}{r}22 \\
143 \\
168 \\
161 \\
189\end{array}$ & $\begin{array}{l}10 \\
34 \\
50 \\
44 \\
63\end{array}$ & $\begin{array}{l}1.8 \\
3.1 \\
3.2 \\
3.6 \\
4.5\end{array}$ & $\begin{array}{l}.62 \\
.80 \\
.62 \\
.71 \\
.44\end{array}$ & $\begin{array}{r}20666 \\
7260 \\
5929 \\
5586 \\
3900\end{array}$ & $\begin{array}{l}3.72 \\
2.92 \\
2.62 \\
2.77 \\
2.60\end{array}$ \\
\hline
\end{tabular}


Table B-1 cont'd

\begin{tabular}{|c|c|c|c|c|c|c|c|c|c|c|c|c|c|c|c|}
\hline $\begin{array}{l}\text { Alun } \\
\text { Dose } \\
\text { mg/L }\end{array}$ & $\begin{array}{l}\text { Time } \\
\text { min. }\end{array}$ & 3.5 & 7.5 & $\begin{array}{l}\text { Ave } \\
12.5\end{array}$ & $\begin{array}{l}\text { lage } \\
17.5\end{array}$ & $\begin{array}{l}\text { DEtCl } \\
22.5\end{array}$ & $\begin{array}{l}081 \\
27.5\end{array}$ & $\begin{array}{l}30, \pi \\
32.5\end{array}$ & $\begin{array}{l}\text { cron } \\
37.5\end{array}$ & 45 & 62.5 & 87.5 & 125 & Total & $\begin{array}{c}\text { Distribution } \\
\text { slope, } \beta\end{array}$ \\
\hline 8.0 & $\begin{array}{r}4.00 \\
13.83 \\
23.67 \\
37.67 \\
52.17\end{array}$ & $\begin{array}{l}8257 \\
1863 \\
1525 \\
1800 \\
1542\end{array}$ & $\begin{array}{r}5364 \\
1082 \\
912 \\
1101 \\
902\end{array}$ & $\begin{array}{r}1812 \\
528 \\
455 \\
496 \\
462\end{array}$ & $\begin{array}{l}801 \\
391 \\
372 \\
347 \\
369\end{array}$ & $\begin{array}{l}373 \\
312 \\
312 \\
265 \\
332\end{array}$ & $\begin{array}{l}166 \\
230 \\
228 \\
190 \\
253\end{array}$ & $\begin{array}{r}90 \\
185 \\
198 \\
165 \\
196\end{array}$ & $\begin{array}{r}34 \\
99 \\
100 \\
94 \\
92\end{array}$ & $\begin{array}{r}46 \\
175 \\
178 \\
180 \\
163\end{array}$ & $\begin{array}{l}15 \\
52 \\
55 \\
55 \\
42\end{array}$ & $\begin{array}{l}2.7 \\
4.4 \\
3.9 \\
3.7 \\
2.2\end{array}$ & $\begin{array}{r}.80 \\
.98 \\
1.16 \\
1.60 \\
.53\end{array}$ & $\begin{array}{r}16963 \\
4923 \\
4340 \\
4699 \\
4355\end{array}$ & $\begin{array}{l}3.48 \\
2.65 \\
2.54 \\
2.57 \\
2.48\end{array}$ \\
\hline 11.0 & $\begin{array}{r}5.10 \\
14.83 \\
24.58 \\
38.83 \\
53.33\end{array}$ & $\begin{array}{l}4396 \\
1545 \\
1661 \\
1296 \\
1363\end{array}$ & $\begin{array}{r}2769 \\
867 \\
971 \\
749 \\
674\end{array}$ & $\begin{array}{r}1115 \\
411 \\
446 \\
365 \\
283\end{array}$ & $\begin{array}{l}615 \\
356 \\
333 \\
295 \\
213\end{array}$ & $\begin{array}{l}361 \\
288 \\
269 \\
259 \\
200\end{array}$ & $\begin{array}{l}184 \\
206 \\
199 \\
201 \\
165\end{array}$ & $\begin{array}{l}124 \\
177 \\
160 \\
175 \\
158\end{array}$ & $\begin{array}{l}57 \\
90 \\
83 \\
89 \\
89\end{array}$ & $\begin{array}{l}96 \\
169 \\
160 \\
174 \\
183\end{array}$ & $\begin{array}{l}26 \\
53 \\
53 \\
51 \\
60\end{array}$ & $\begin{array}{l}4.0 \\
3.4 \\
2.8 \\
2.9 \\
2.9\end{array}$ & $\begin{array}{l}.62 \\
.80 \\
.44 \\
.53 \\
.80\end{array}$ & $\begin{array}{l}9747 \\
4165 \\
4337 \\
3658 \\
3392\end{array}$ & $\begin{array}{l}3.15 \\
2.59 \\
2.52 \\
2.40 \\
2.50\end{array}$ \\
\hline 15.0 & $\begin{array}{r}6.30 \\
16.33 \\
26.00 \\
40.00 \\
54.67\end{array}$ & $\begin{array}{l}2838 \\
1704 \\
1162 \\
1370 \\
1421\end{array}$ & $\begin{array}{r}1718 \\
948 \\
628 \\
1016 \\
713\end{array}$ & $\begin{array}{l}727 \\
432 \\
298 \\
327 \\
306\end{array}$ & $\begin{array}{l}789 \\
332 \\
250 \\
270 \\
245\end{array}$ & $\begin{array}{l}348 \\
269 \\
231 \\
237 \\
210\end{array}$ & $\begin{array}{l}216 \\
197 \\
195 \\
187 \\
164\end{array}$ & $\begin{array}{l}163 \\
174 \\
174 \\
165 \\
159\end{array}$ & $\begin{array}{l}76 \\
90 \\
91 \\
85 \\
92\end{array}$ & $\begin{array}{l}139 \\
168 \\
168 \\
180 \\
188\end{array}$ & $\begin{array}{l}44 \\
55 \\
50 \\
57 \\
61\end{array}$ & $\begin{array}{l}3.3 \\
5.1 \\
4.5 \\
3.2 \\
4.1\end{array}$ & $\begin{array}{r}1.10 \\
1.33 \\
.44 \\
.98 \\
1.16\end{array}$ & $\begin{array}{l}6762 \\
4376 \\
3252 \\
3898 \\
3563\end{array}$ & $\begin{array}{l}2.89 \\
2.51 \\
2.55 \\
2.33 \\
2.43\end{array}$ \\
\hline
\end{tabular}


Table B-2 - Remaining Number of Particles per ml for $\mathrm{G}=20 \mathrm{~s}^{-1}$, and pH-7.00

\begin{tabular}{|c|c|c|c|c|c|c|c|c|c|c|c|c|c|c|c|}
\hline $\begin{array}{l}\text { Alum } \\
\text { Dose } \\
\text { mg/L }\end{array}$ & $\begin{array}{l}\text { Time } \\
\text { min. }\end{array}$ & 3.5 & 7.5 & $\begin{array}{r}\text { Aver } \\
12.5\end{array}$ & 17.5 & $\begin{array}{l}\text { artic } \\
22.5\end{array}$ & $\begin{array}{r}1081 \\
27.5\end{array}$ & $\begin{array}{l}3 . M \\
32.5\end{array}$ & $\begin{array}{l}\text { cron } \\
37.5\end{array}$ & 545 & 62.5 & 87.5 & 125 & Total & $\begin{array}{c}\text { Distribution } \\
\text { slope, } \beta\end{array}$ \\
\hline 5.0 & $\begin{array}{r}3.50 \\
12.75 \\
23.50 \\
36.00 \\
53.25\end{array}$ & $\begin{array}{r}10146 \\
4964 \\
2735 \\
1955 \\
1597\end{array}$ & $\begin{array}{r}6692 \\
3251 \\
1813 \\
1108 \\
971\end{array}$ & $\begin{array}{r}2309 \\
1470 \\
858 \\
523 \\
499\end{array}$ & $\begin{array}{l}879 \\
930 \\
578 \\
352 \\
386\end{array}$ & $\begin{array}{l}340 \\
586 \\
408 \\
244 \\
295\end{array}$ & $\begin{array}{l}121 \\
311 \\
248 \\
166 \\
198\end{array}$ & $\begin{array}{r}48 \\
182 \\
189 \\
132 \\
157\end{array}$ & $\begin{array}{l}19 \\
86 \\
88 \\
68 \\
81\end{array}$ & $\begin{array}{l}23 \\
113 \\
161 \\
162 \\
165\end{array}$ & $\begin{array}{r}8 \\
26 \\
54 \\
74 \\
66\end{array}$ & $\begin{array}{l}1.0 \\
2.0 \\
2.3 \\
3.6 \\
3.0\end{array}$ & $\begin{array}{l}0.27 \\
0.09 \\
0.00 \\
0.00 \\
0.27\end{array}$ & $\begin{array}{r}20886 \\
11920 \\
7133 \\
4788 \\
4416\end{array}$ & $\begin{array}{l}3.91 \\
3.01 \\
2.43 \\
2.19 \\
2.56\end{array}$ \\
\hline
\end{tabular}


Table B-2 Cont'd

\begin{tabular}{|c|c|c|c|c|c|c|c|c|c|c|c|c|c|c|c|}
\hline \multirow{2}{*}{$\begin{array}{l}\text { Alum } \\
\text { Dose } \\
\mathrm{mg} / \mathrm{L}\end{array}$} & \multirow[b]{2}{*}{$\begin{array}{l}\text { Time } \\
\text { min. }\end{array}$} & \multicolumn{13}{|c|}{ Average Particle s12e, Mcrons } & \multirow[b]{2}{*}{$\begin{array}{c}\text { Distribution } \\
\text { slope, } \beta\end{array}$} \\
\hline & & 3.5 & 7.5 & 12.5 & 17.5 & 22.5 & 27.5 & 32.5 & 37.5 & 545 & 62.5 & 87.5 & 125 & Total & \\
\hline 15.0 & $\begin{array}{r}0.50 \\
8.75 \\
19.25 \\
31.00 \\
47.75\end{array}$ & $\begin{array}{r}10125 \\
2160 \\
1678 \\
1183 \\
1433\end{array}$ & $\begin{array}{r}6255 \\
1143 \\
1024 \\
676 \\
806\end{array}$ & $\begin{array}{r}2088 \\
472 \\
512 \\
289 \\
315\end{array}$ & $\begin{array}{l}955 \\
299 \\
341 \\
210 \\
196\end{array}$ & $\begin{array}{l}470 \\
219 \\
214 \\
152 \\
149\end{array}$ & $\begin{array}{l}209 \\
147 \\
124 \\
104 \\
104\end{array}$ & $\begin{array}{r}103 \\
120 \\
96 \\
91 \\
87\end{array}$ & $\begin{array}{l}38 \\
65 \\
46 \\
53 \\
53\end{array}$ & $\begin{array}{r}48 \\
151 \\
98 \\
139 \\
140\end{array}$ & $\begin{array}{r}15 \\
98 \\
65 \\
90 \\
107\end{array}$ & $\begin{array}{r}2.4 \\
9.4 \\
7.7 \\
6.9 \\
13.2\end{array}$ & $\begin{array}{l}0.80 \\
1.07 \\
0.53 \\
0.44 \\
1.24\end{array}$ & $\begin{array}{r}20308 \\
4884 \\
4207 \\
2995 \\
3405\end{array}$ & $\begin{array}{l}3.59 \\
2.54 \\
2.44 \\
2.45 \\
2.08\end{array}$ \\
\hline
\end{tabular}


Table B-3 - Remaining Number of Particles per ml for G-65 5-1, and pH=7.00

\begin{tabular}{|c|c|c|c|c|c|c|c|c|c|c|c|c|c|c|c|}
\hline $\begin{array}{l}\text { Alum } \\
\text { Dose } \\
\mathrm{mg} / \mathrm{L}\end{array}$ & $\begin{array}{l}\text { Time } \\
\text { min. }\end{array}$ & 3.5 & 7.5 & $\begin{array}{r}\text { Aver } \\
12.5\end{array}$ & $\begin{array}{l}\text { rage } \\
17.5\end{array}$ & $\begin{array}{l}\text { ArEIC } \\
22.5\end{array}$ & $\begin{array}{r}1031 \\
27.5\end{array}$ & $\begin{array}{l}6, k] \\
32.5\end{array}$ & $\begin{array}{l}\text { crone } \\
37.5\end{array}$ & $\begin{array}{l}85 \\
48\end{array}$ & 62.5 & 87.5 & 125 & Total & $\begin{array}{c}\text { Distribution } \\
\text { slope, } \beta\end{array}$ \\
\hline 5.0 & $\begin{array}{r}3.50 \\
13.25 \\
23.00 \\
33.00 \\
46.25\end{array}$ & $\begin{array}{l}7852 \\
2297 \\
1533 \\
1390 \\
1219\end{array}$ & $\begin{array}{r}5355 \\
1470 \\
850 \\
763 \\
691\end{array}$ & $\begin{array}{r}1965 \\
696 \\
367 \\
333 \\
328\end{array}$ & $\begin{array}{l}844 \\
496 \\
256 \\
251 \\
243\end{array}$ & $\begin{array}{l}371 \\
375 \\
210 \\
198 \\
207\end{array}$ & $\begin{array}{l}140 \\
255 \\
162 \\
148 \\
157\end{array}$ & $\begin{array}{r}64 \\
197 \\
145 \\
137 \\
154\end{array}$ & $\begin{array}{r}20 \\
106 \\
92 \\
84 \\
93\end{array}$ & $\begin{array}{r}29 \\
197 \\
204 \\
214 \\
209\end{array}$ & $\begin{array}{r}8 \\
73 \\
104 \\
104 \\
92\end{array}$ & $\begin{array}{l}1.6 \\
4.4 \\
6.6 \\
4.0 \\
4.2\end{array}$ & $\begin{array}{l}0.53 \\
0.71 \\
0.36 \\
0.36 \\
0.36\end{array}$ & $\begin{array}{r}16652 \\
6167 \\
3929 \\
3626 \\
3397\end{array}$ & $\begin{array}{l}3.56 \\
2.74 \\
2.54 \\
2.08 \\
2.29\end{array}$ \\
\hline
\end{tabular}


Table B-3 Cont'd

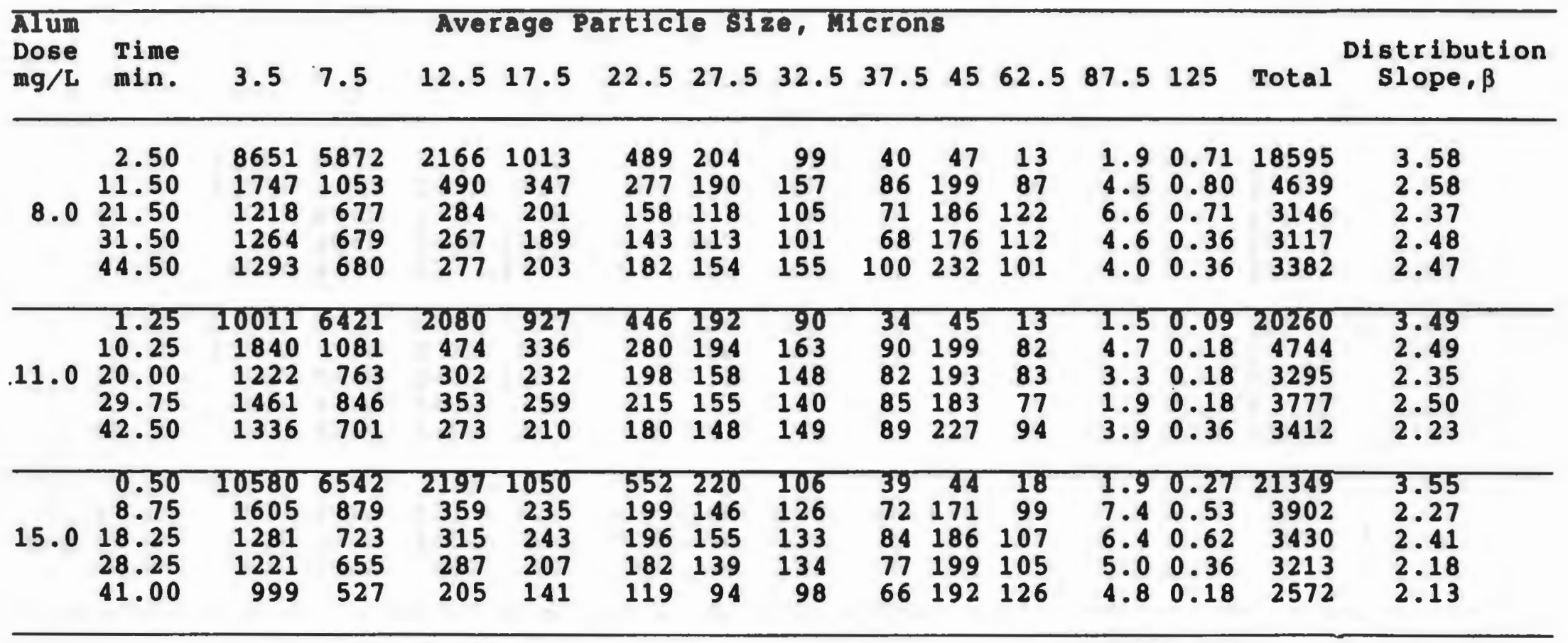


Table B-4 - Remaining Number of Particles per ml for $G=40^{\circ} \mathrm{s}^{-1}, 8.0 \mathrm{mg} / \mathrm{L} \mathrm{Alum}$

\begin{tabular}{|c|c|c|c|c|c|c|c|c|c|c|c|c|c|c|c|}
\hline $\mathrm{pH}$ & $\begin{array}{l}\text { Time } \\
\text { min. }\end{array}$ & 3.5 & 7.5 & $\begin{array}{l}\text { Avar } \\
12.5\end{array}$ & $\begin{array}{l}\text { rage } \\
17.5\end{array}$ & $\begin{array}{l}\text { articl } \\
22.5\end{array}$ & $\begin{array}{r}1.81 \\
27.5\end{array}$ & $\begin{array}{l}6, \mathrm{H} \\
32.5\end{array}$ & $\begin{array}{l}\text { cront } \\
37.5\end{array}$ & 45 & 62.5 & 87.5 & 125 & Tota1 & $\begin{array}{c}\text { Distribution } \\
\text { slope, } \beta\end{array}$ \\
\hline 4.0 & $\begin{array}{r}0.50 \\
8.50 \\
19.50 \\
34.50 \\
57.50\end{array}$ & $\begin{array}{r}11969 \\
11352 \\
9079 \\
7374 \\
6024\end{array}$ & $\begin{array}{l}6000 \\
7581 \\
6542 \\
5843 \\
4495\end{array}$ & $\begin{array}{l}1314 \\
2167 \\
2278 \\
2669 \\
2231\end{array}$ & $\begin{array}{r}460 \\
793 \\
878 \\
1307 \\
1323\end{array}$ & $\begin{array}{l}182 \\
304 \\
302 \\
523 \\
629\end{array}$ & $\begin{array}{r}82 \\
120 \\
103 \\
160 \\
215\end{array}$ & $\begin{array}{l}42 \\
59 \\
48 \\
80 \\
96\end{array}$ & $\begin{array}{l}17 \\
22 \\
17 \\
30 \\
31\end{array}$ & $\begin{array}{l}28 \\
41 \\
27 \\
46 \\
48\end{array}$ & $\begin{array}{l}12 \\
20 \\
10 \\
19 \\
21\end{array}$ & $\begin{array}{l}3.0 \\
2.8 \\
1.4 \\
2.0 \\
2.5\end{array}$ & $\begin{array}{l}0.09 \\
0.44 \\
0.09 \\
0.62 \\
0.36\end{array}$ & $\begin{array}{l}20108 \\
22462 \\
19286 \\
18053 \\
15116\end{array}$ & $\begin{array}{l}3.47 \\
3.54 \\
3.62 \\
3.56 \\
3.53\end{array}$ \\
\hline 5.0 & $\begin{array}{r}1.50 \\
9.75 \\
20.75 \\
35.75 \\
58.75\end{array}$ & $\begin{array}{r}11970 \\
10370 \\
7985 \\
6002 \\
4103\end{array}$ & $\begin{array}{l}6918 \\
7176 \\
5995 \\
4218 \\
3035\end{array}$ & $\begin{array}{l}1848 \\
2331 \\
2485 \\
1665 \\
1455\end{array}$ & $\begin{array}{r}726 \\
853 \\
1107 \\
779 \\
843\end{array}$ & $\begin{array}{l}311 \\
337 \\
417 \\
287 \\
418\end{array}$ & $\begin{array}{r}123 \\
130 \\
132 \\
87 \\
143\end{array}$ & $\begin{array}{l}66 \\
66 \\
59 \\
39 \\
52\end{array}$ & $\begin{array}{l}28 \\
29 \\
22 \\
12 \\
16\end{array}$ & $\begin{array}{l}43 \\
45 \\
36 \\
18 \\
22\end{array}$ & $\begin{array}{r}24 \\
20 \\
14 \\
9 \\
8\end{array}$ & $\begin{array}{l}3.6 \\
4.3 \\
2.0 \\
1.0 \\
1.1\end{array}$ & $\begin{array}{l}0.36 \\
0.53 \\
0.53 \\
0.44 \\
0.36\end{array}$ & $\begin{array}{l}22061 \\
21362 \\
18254 \\
13118 \\
10098\end{array}$ & $\begin{array}{l}3.46 \\
3.56 \\
3.66 \\
3.64 \\
3.46\end{array}$ \\
\hline 6.0 & $\begin{array}{r}2.25 \\
22.25 \\
37.00 \\
60.50\end{array}$ & $\begin{array}{r}11139 \\
3579 \\
2918 \\
2672\end{array}$ & $\begin{array}{l}5477 \\
2414 \\
2065 \\
1801\end{array}$ & $\begin{array}{r}1169 \\
1210 \\
1054 \\
962\end{array}$ & $\begin{array}{l}423 \\
880 \\
752 \\
781\end{array}$ & $\begin{array}{l}199 \\
611 \\
522 \\
596\end{array}$ & $\begin{array}{r}78 \\
346 \\
290 \\
364\end{array}$ & $\begin{array}{r}40 \\
212 \\
186 \\
239\end{array}$ & $\begin{array}{l}17 \\
91 \\
76 \\
95\end{array}$ & $\begin{array}{r}28 \\
124 \\
99 \\
125\end{array}$ & $\begin{array}{l}13 \\
35 \\
27 \\
32\end{array}$ & $\begin{array}{l}1.7 \\
5.1 \\
2.3 \\
3.1\end{array}$ & $\begin{array}{l}0.27 \\
0.89 \\
0.44 \\
0.36\end{array}$ & $\begin{array}{r}18585 \\
9509 \\
7991 \\
7670\end{array}$ & $\begin{array}{l}3.58 \\
2.99 \\
2.91 \\
3.06\end{array}$ \\
\hline
\end{tabular}




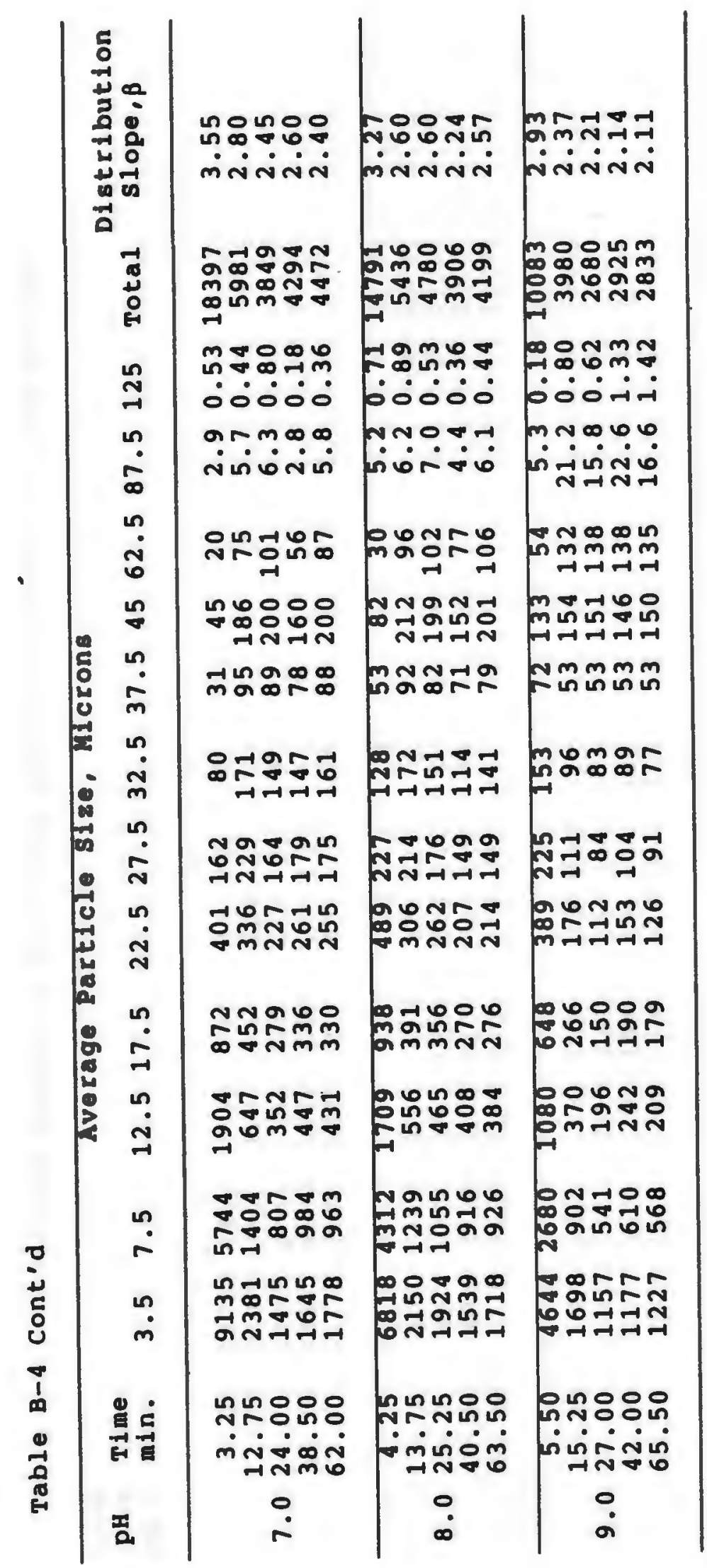


Table B-5 - Remaining Number of Particles per ml for $G^{-}=40 \mathrm{~s}^{-1}$, and $\mathrm{pH}=6.00$

\begin{tabular}{|c|c|c|c|c|c|c|c|c|c|c|c|c|c|c|c|}
\hline $\begin{array}{l}\text { MIun } \\
\text { Dose } \\
\text { mg/L }\end{array}$ & $\begin{array}{l}\text { Time } \\
\text { min. }\end{array}$ & 3.5 & 7.5 & $\begin{array}{l}\text { Aver } \\
12.5\end{array}$ & $\begin{array}{c}\text { rage } \bar{P} \\
17.5\end{array}$ & $\begin{array}{l}\text { articl } \\
22.5\end{array}$ & $\begin{array}{r}1681 \\
27.5\end{array}$ & $\begin{array}{l}\pi, \pi \\
32.5\end{array}$ & $\begin{array}{l}\text { cron: } \\
37.5\end{array}$ & 45 & 62.5 & 87.5 & 125 & Total & $\begin{array}{c}\text { D1stribution } \\
\text { slope, } \beta\end{array}$ \\
\hline 0.0 & $\begin{array}{r}0.00 \\
1.00 \\
8.25 \\
18.50 \\
31.50\end{array}$ & $\begin{array}{l}11720 \\
10387 \\
11001 \\
11272 \\
11638\end{array}$ & $\begin{array}{l}7349 \\
5616 \\
6173 \\
6600 \\
7174\end{array}$ & $\begin{array}{l}2103 \\
1377 \\
1571 \\
1778 \\
1945\end{array}$ & $\begin{array}{l}791 \\
520 \\
603 \\
667 \\
714\end{array}$ & $\begin{array}{l}340 \\
224 \\
249 \\
270 \\
301\end{array}$ & $\begin{array}{r}120 \\
86 \\
94 \\
104 \\
109\end{array}$ & $\begin{array}{l}60 \\
44 \\
45 \\
47 \\
55\end{array}$ & $\begin{array}{l}23 \\
17 \\
19 \\
17 \\
20\end{array}$ & $\begin{array}{l}33 \\
27 \\
24 \\
26 \\
28\end{array}$ & $\begin{array}{l}15 \\
14 \\
11 \\
10 \\
13\end{array}$ & $\begin{array}{l}3.3 \\
3.0 \\
2.0 \\
1.7 \\
2.6\end{array}$ & $\begin{array}{l}0.98 \\
0.62 \\
0.44 \\
0.71 \\
0.71\end{array}$ & $\begin{array}{l}22560 \\
18316 \\
19792 \\
20795 \\
22000\end{array}$ & $\begin{array}{l}3.60 \\
3.45 \\
3.72 \\
3.61 \\
3.64\end{array}$ \\
\hline 2.0 & $\begin{array}{r}1.75 \\
9.25 \\
19.75 \\
32.45 \\
47.25\end{array}$ & $\begin{array}{r}11149 \\
9259 \\
6807 \\
4847 \\
4177\end{array}$ & $\begin{array}{l}7074 \\
6777 \\
5896 \\
4133 \\
3330\end{array}$ & $\begin{array}{l}2092 \\
2280 \\
2670 \\
2072 \\
1808\end{array}$ & $\begin{array}{r}825 \\
856 \\
1207 \\
1109 \\
1229\end{array}$ & $\begin{array}{l}344 \\
323 \\
463 \\
484 \\
749\end{array}$ & $\begin{array}{l}123 \\
118 \\
140 \\
150 \\
316\end{array}$ & $\begin{array}{r}58 \\
51 \\
63 \\
53 \\
152\end{array}$ & $\begin{array}{l}21 \\
22 \\
21 \\
19 \\
46\end{array}$ & $\begin{array}{l}32 \\
35 \\
34 \\
23 \\
46\end{array}$ & $\begin{array}{l}11 \\
14 \\
13 \\
10 \\
16\end{array}$ & $\begin{array}{l}3.5 \\
1.1 \\
2.8 \\
1.8 \\
2.0\end{array}$ & $\begin{array}{l}0.71 \\
0.89 \\
0.98 \\
0.18 \\
0.62\end{array}$ & $\begin{array}{l}21734 \\
19737 \\
17318 \\
12902 \\
11871\end{array}$ & $\begin{array}{l}3.60 \\
3.71 \\
3.50 \\
3.37 \\
3.42\end{array}$ \\
\hline 5.0 & $\begin{array}{r}2.75 \\
10.75 \\
21.25 \\
34.25 \\
47.75\end{array}$ & $\begin{array}{r}10408 \\
8867 \\
6808 \\
4377 \\
2639\end{array}$ & $\begin{array}{l}5948 \\
6707 \\
5670 \\
3425 \\
1904\end{array}$ & $\begin{array}{r}1559 \\
2546 \\
2715 \\
1776 \\
968\end{array}$ & $\begin{array}{r}596 \\
1061 \\
1360 \\
1076 \\
686\end{array}$ & $\begin{array}{l}236 \\
420 \\
563 \\
601 \\
469\end{array}$ & $\begin{array}{r}86 \\
139 \\
182 \\
250 \\
269\end{array}$ & $\begin{array}{r}43 \\
64 \\
72 \\
119 \\
156\end{array}$ & $\begin{array}{l}16 \\
21 \\
26 \\
36 \\
62\end{array}$ & $\begin{array}{l}27 \\
34 \\
33 \\
43 \\
76\end{array}$ & $\begin{array}{l}13 \\
14 \\
14 \\
12 \\
15\end{array}$ & $\begin{array}{l}2.6 \\
2.3 \\
3.0 \\
3.0 \\
1.4\end{array}$ & $\begin{array}{l}0.53 \\
1.07 \\
0.89 \\
0.89 \\
0.27\end{array}$ & $\begin{array}{r}18939 \\
19876 \\
17446 \\
11717 \\
7244\end{array}$ & $\begin{array}{l}3.51 \\
3.59 \\
3.51 \\
3.31 \\
3.07\end{array}$ \\
\hline
\end{tabular}


Table B-5 cont'd

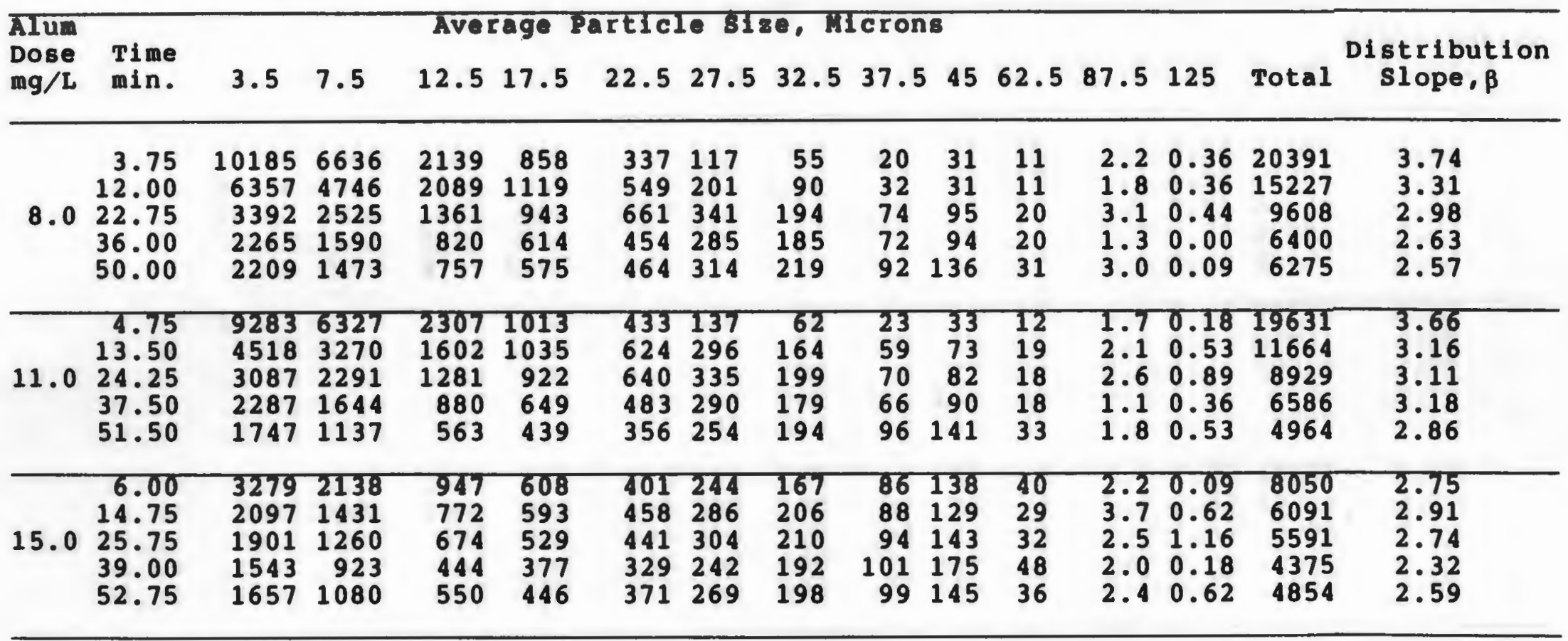


Table B-6 - Remaining Number of Particles per ml for $\mathrm{G}=40 \mathrm{~s}^{-1}$, and $\mathrm{pH}=8.00$

\begin{tabular}{|c|c|c|c|c|c|c|c|c|c|c|c|c|c|c|c|}
\hline $\begin{array}{l}\text { Alum } \\
\text { Dose } \\
\mathrm{mg} / \mathrm{L}\end{array}$ & $\begin{array}{l}\text { Time } \\
\text { min. }\end{array}$ & 3.5 & 7.5 & $\begin{array}{l}\text { Ave } \\
12.5\end{array}$ & $\begin{array}{l}\text { rage } \\
17.5\end{array}$ & $\begin{array}{l}\text { atic] } \\
22.5\end{array}$ & $\begin{array}{l}\text { le } \$ 12 \\
27.5\end{array}$ & $\begin{array}{l}\text { e, M } \\
32.5\end{array}$ & $\begin{array}{l}\text { crons } \\
37.5\end{array}$ & $\begin{array}{l}18 \\
45\end{array}$ & 62.5 & 87.5 & 125 & Tota1 & $\begin{array}{c}\text { Distribution } \\
\text { slope, } \beta\end{array}$ \\
\hline 0.0 & $\begin{array}{r}0.00 \\
0.25 \\
7.25 \\
13.50 \\
36.75\end{array}$ & $\begin{array}{r}11720 \\
10260 \\
10787 \\
9629 \\
8833\end{array}$ & $\begin{array}{l}7349 \\
5906 \\
6731 \\
7140 \\
7387\end{array}$ & $\begin{array}{l}2103 \\
1635 \\
1885 \\
2307 \\
2779\end{array}$ & $\begin{array}{r}791 \\
615 \\
701 \\
866 \\
1007\end{array}$ & $\begin{array}{l}340 \\
260 \\
293 \\
375 \\
368\end{array}$ & $\begin{array}{l}120 \\
102 \\
114 \\
127 \\
126\end{array}$ & $\begin{array}{l}60 \\
52 \\
53 \\
64 \\
56\end{array}$ & $\begin{array}{l}23 \\
22 \\
19 \\
21 \\
21\end{array}$ & $\begin{array}{l}33 \\
30 \\
34 \\
35 \\
32\end{array}$ & $\begin{array}{l}15 \\
14 \\
14 \\
14 \\
15\end{array}$ & $\begin{array}{l}3.3 \\
3.4 \\
2.8 \\
2.8 \\
3.4\end{array}$ & $\begin{array}{l}0.98 \\
0.98 \\
0.80 \\
0.27 \\
0.36\end{array}$ & $\begin{array}{l}22560 \\
18900 \\
20635 \\
20581 \\
20627\end{array}$ & $\begin{array}{l}3.60 \\
3.50 \\
3.59 \\
3.73 \\
3.53\end{array}$ \\
\hline 2.0 & $\begin{array}{r}0.75 \\
8.25 \\
22.00 \\
35.50 \\
60.00\end{array}$ & $\begin{array}{r}10495 \\
7395 \\
3733 \\
2402 \\
2366\end{array}$ & $\begin{array}{l}6670 \\
5732 \\
2749 \\
1644 \\
1644\end{array}$ & $\begin{array}{r}2015 \\
2390 \\
1454 \\
877 \\
871\end{array}$ & $\begin{array}{r}766 \\
1113 \\
999 \\
648 \\
629\end{array}$ & $\begin{array}{l}312 \\
484 \\
657 \\
489 \\
477\end{array}$ & $\begin{array}{l}118 \\
163 \\
345 \\
288 \\
297\end{array}$ & $\begin{array}{r}60 \\
71 \\
196 \\
207 \\
214\end{array}$ & $\begin{array}{l}22 \\
25 \\
73 \\
95 \\
99\end{array}$ & $\begin{array}{r}34 \\
39 \\
87 \\
140 \\
155\end{array}$ & $\begin{array}{l}15 \\
17 \\
23 \\
36 \\
41\end{array}$ & $\begin{array}{l}3.0 \\
2.5 \\
2.8 \\
4.3 \\
3.5\end{array}$ & $\begin{array}{l}0.98 \\
0.53 \\
1.24 \\
0.71 \\
1.07\end{array}$ & $\begin{array}{r}20511 \\
17431 \\
10318 \\
6832 \\
6799\end{array}$ & $\begin{array}{l}3.57 \\
3.58 \\
3.09 \\
2.88 \\
2.82\end{array}$ \\
\hline 5.0 & $\begin{array}{r}1.75 \\
9.50 \\
20.50 \\
34.00 \\
48.25\end{array}$ & $\begin{array}{l}9481 \\
4282 \\
2012 \\
1431 \\
1298\end{array}$ & $\begin{array}{r}5875 \\
2884 \\
1267 \\
893 \\
793\end{array}$ & $\begin{array}{r}1699 \\
1380 \\
625 \\
427 \\
382\end{array}$ & $\begin{array}{l}638 \\
898 \\
422 \\
303 \\
277\end{array}$ & $\begin{array}{l}275 \\
595 \\
332 \\
232 \\
212\end{array}$ & $\begin{array}{l}105 \\
309 \\
217 \\
169 \\
163\end{array}$ & $\begin{array}{r}49 \\
173 \\
177 \\
140 \\
134\end{array}$ & $\begin{array}{l}18 \\
73 \\
93 \\
76 \\
77\end{array}$ & $\begin{array}{r}31 \\
92 \\
182 \\
166 \\
165\end{array}$ & $\begin{array}{l}13 \\
23 \\
65 \\
75 \\
67\end{array}$ & $\begin{array}{l}2.2 \\
5.0 \\
5.6 \\
7.0 \\
4.6\end{array}$ & $\begin{array}{l}1.07 \\
0.62 \\
1.42 \\
0.53 \\
0.62\end{array}$ & $\begin{array}{r}18187 \\
10713 \\
5398 \\
3919 \\
3572\end{array}$ & $\begin{array}{l}3.54 \\
3.16 \\
2.59 \\
2.56 \\
2.55\end{array}$ \\
\hline
\end{tabular}


Table B-6 Cont'd

\begin{tabular}{|c|c|c|c|c|c|c|c|c|c|c|c|c|c|c|c|}
\hline $\begin{array}{l}\text { Alum } \\
\text { Dose } \\
\text { mg/L }\end{array}$ & $\begin{array}{l}\text { Time } \\
\text { min. }\end{array}$ & 3.5 & 7.5 & $\begin{array}{r}\text { Ave } \\
12.5\end{array}$ & $\begin{array}{l}\text { rage } \\
17.5\end{array}$ & $\begin{array}{r}\text { articl } \\
22.5\end{array}$ & $\begin{array}{r}\text { le SI } \\
27.5\end{array}$ & $\begin{array}{l}\mathrm{Ze}, \mathrm{M} \\
32.5\end{array}$ & cron & 545 & 62.5 & 87.5 & 125 & Total & $\begin{array}{c}\text { Distribution } \\
\text { slope, } \beta\end{array}$ \\
\hline 15.0 & $\begin{array}{r}4.75 \\
13.00 \\
16.50 \\
29.00 \\
43.25\end{array}$ & $\begin{array}{r}3832 \\
1426 \\
1240 \\
1026 \\
931\end{array}$ & $\begin{array}{r}2372 \\
807 \\
723 \\
551 \\
483\end{array}$ & $\begin{array}{l}951 \\
345 \\
308 \\
221 \\
177\end{array}$ & $\begin{array}{l}529 \\
225 \\
202 \\
153 \\
121\end{array}$ & $\begin{array}{r}327 \\
167 \\
163 \\
115 \\
97\end{array}$ & $\begin{array}{r}182 \\
117 \\
114 \\
85 \\
66\end{array}$ & $\begin{array}{r}119 \\
101 \\
92 \\
78 \\
60\end{array}$ & $\begin{array}{l}57 \\
53 \\
58 \\
46 \\
38\end{array}$ & $\begin{array}{l}109 \\
146 \\
127 \\
131 \\
114\end{array}$ & $\begin{array}{r}45 \\
94 \\
88 \\
98 \\
100\end{array}$ & $\begin{array}{r}3.7 \\
8.7 \\
6.8 \\
9.4 \\
12.3\end{array}$ & $\begin{array}{l}0.53 \\
0.36 \\
0.44 \\
0.71 \\
0.53\end{array}$ & $\begin{array}{l}9129 \\
3489 \\
3123 \\
2513 \\
2200\end{array}$ & $\begin{array}{l}2.88 \\
2.30 \\
2.50 \\
2.29 \\
1.95\end{array}$ \\
\hline
\end{tabular}




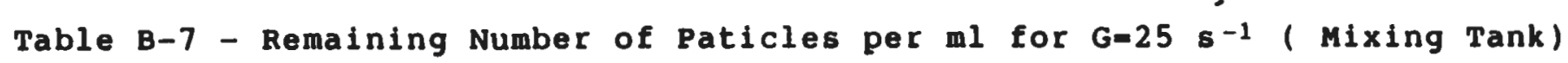

\begin{tabular}{|c|c|c|c|c|c|c|c|c|c|c|c|c|c|c|}
\hline $\begin{array}{l}\text { Time } \\
\text { min. }\end{array}$ & 3.5 & 7.5 & 12.5 & $\begin{array}{l}\text { Aver } \\
17.5\end{array}$ & $\begin{array}{l}e .5 \\
2.5\end{array}$ & 7 & $\begin{array}{l}51 \\
2.5\end{array}$ & 37. & 5 & 62.5 & 587.5 & 125 & Total & $\begin{array}{l}\text { Distribution } \\
\text { slope, } \beta\end{array}$ \\
\hline $\begin{array}{r}0.00 \\
0.50 \\
3.00 \\
5.00 \\
6.75 \\
9.75 \\
11.50 \\
15.25 \\
17.50 \\
22.75 \\
25.00 \\
28.00 \\
30.00 \\
35.00 \\
45.00 \\
50.00 \\
55.25 \\
60.00 \\
70.00 \\
90.25\end{array}$ & $\begin{array}{l}8503 \\
7879 \\
6862 \\
6262 \\
5084 \\
4034 \\
3151 \\
2040 \\
1835 \\
1670 \\
1536 \\
1446 \\
1265 \\
1344 \\
1241 \\
1204 \\
1085 \\
1242 \\
1085 \\
993\end{array}$ & $\begin{array}{r}5191 \\
5222 \\
4844 \\
4265 \\
3595 \\
2750 \\
2016 \\
1281 \\
1155 \\
1022 \\
960 \\
868 \\
740 \\
753 \\
705 \\
722 \\
611 \\
702 \\
618 \\
585\end{array}$ & $\begin{array}{r}1623 \\
1688 \\
1708 \\
1646 \\
1605 \\
1193 \\
904 \\
616 \\
572 \\
508 \\
469 \\
428 \\
361 \\
355 \\
320 \\
357 \\
278 \\
330 \\
292 \\
276\end{array}$ & $\begin{array}{r}758 \\
802 \\
791 \\
861 \\
1013 \\
742 \\
600 \\
467 \\
426 \\
398 \\
359 \\
341 \\
304 \\
326 \\
234 \\
301 \\
250 \\
264 \\
249 \\
215\end{array}$ & $\begin{array}{l}355 \\
384 \\
345 \\
470 \\
554 \\
453 \\
399 \\
362 \\
331 \\
301 \\
283 \\
264 \\
246 \\
247 \\
189 \\
253 \\
190 \\
203 \\
202 \\
193\end{array}$ & $\begin{array}{l}154 \\
163 \\
135 \\
200 \\
249 \\
237 \\
221 \\
229 \\
207 \\
211 \\
200 \\
182 \\
185 \\
159 \\
137 \\
198 \\
146 \\
145 \\
169 \\
152\end{array}$ & $\begin{array}{l}81 \\
86 \\
76 \\
110 \\
135 \\
149 \\
161 \\
171 \\
159 \\
163 \\
160 \\
145 \\
153 \\
138 \\
124 \\
164 \\
131 \\
118 \\
145 \\
132\end{array}$ & $\begin{array}{l}34 \\
34 \\
30 \\
45 \\
52 \\
65 \\
76 \\
86 \\
79 \\
84 \\
86 \\
79 \\
84 \\
78 \\
75 \\
90 \\
76 \\
70 \\
85 \\
77\end{array}$ & $\begin{array}{r}57 \\
60 \\
52 \\
72 \\
78 \\
116 \\
143 \\
164 \\
159 \\
168 \\
174 \\
170 \\
178 \\
181 \\
176 \\
186 \\
180 \\
163 \\
171 \\
164\end{array}$ & $\begin{array}{l}30 \\
28 \\
27 \\
30 \\
31 \\
44 \\
59 \\
68 \\
63 \\
69 \\
78 \\
79 \\
82 \\
85 \\
88 \\
76 \\
95 \\
77 \\
77 \\
77\end{array}$ & $\begin{array}{r}4.62 \\
4.80 \\
5.33 \\
5.96 \\
5.78 \\
7.20 \\
7.02 \\
8.62 \\
7.73 \\
8.44 \\
8.62 \\
9.51 \\
9.07 \\
8.53 \\
7.56 \\
8.71 \\
10.22 \\
7.02 \\
7.91 \\
6.49\end{array}$ & $\begin{array}{l}1.51 \\
2.04 \\
1.42 \\
1.24 \\
1.24 \\
1.60 \\
1.33 \\
1.60 \\
1.96 \\
1.33 \\
1.33 \\
2.49 \\
1.33 \\
2.40 \\
1.24 \\
1.33 \\
1.69 \\
1.24 \\
1.51 \\
1.24\end{array}$ & $\begin{array}{r}16791 \\
16351 \\
14876 \\
13968 \\
12403 \\
9792 \\
7737 \\
5494 \\
4997 \\
4603 \\
4315 \\
4013 \\
3609 \\
3678 \\
3298 \\
3560 \\
3054 \\
3325 \\
3104 \\
2865\end{array}$ & $\begin{array}{l}3.26 \\
3.22 \\
3.24 \\
3.21 \\
3.11 \\
2.89 \\
2.77 \\
2.56 \\
2.50 \\
2.49 \\
2.43 \\
2.31 \\
2.33 \\
2.29 \\
2.32 \\
2.33 \\
2.19 \\
2.36 \\
2.24 \\
2.28\end{array}$ \\
\hline
\end{tabular}


Table B-8 - Remaining Number of Particles per ml for G=50 $\mathrm{s}^{-1}$ ( Mixing Tank)

\begin{tabular}{|c|c|c|c|c|c|c|c|c|c|c|c|c|c|c|}
\hline $\begin{array}{l}\text { Time } \\
\text { min. }\end{array}$ & 3.5 & 7.5 & 12.5 & $\begin{array}{l}\text { Avera } \\
17.5\end{array}$ & $\begin{array}{r}g e \\
22.5\end{array}$ & $\begin{array}{l}\text { irtic } \\
27.5\end{array}$ & $\begin{array}{c}1 \mathrm{e} 512 \\
32.5\end{array}$ & $\begin{array}{l}2 \mathrm{e}, \mathrm{M} \\
37.5\end{array}$ & $\begin{array}{c}1 \text { Cro } \\
45\end{array}$ & $\begin{array}{l}\text { ng } \\
62.5\end{array}$ & 87.5 & 125 & Total & $\begin{array}{c}\text { Distribution } \\
\text { slope } \beta\end{array}$ \\
\hline $\begin{array}{r}0.00 \\
0.75 \\
2.75 \\
4.25 \\
6.25 \\
7.75 \\
10.25 \\
12.75 \\
15.25 \\
18.25 \\
23.25 \\
28.50 \\
33.75 \\
38.75 \\
43.75 \\
54.75 \\
68.75 \\
79.75 \\
88.75\end{array}$ & $\begin{array}{l}9329 \\
8765 \\
7412 \\
6028 \\
4513 \\
3315 \\
2307 \\
1894 \\
1392 \\
1324 \\
1214 \\
1128 \\
1196 \\
1218 \\
1686 \\
1328 \\
1161 \\
1302 \\
1300\end{array}$ & $\begin{array}{r}5668 \\
5772 \\
5100 \\
4308 \\
3192 \\
1771 \\
1515 \\
1276 \\
910 \\
818 \\
697 \\
694 \\
729 \\
762 \\
953 \\
762 \\
681 \\
768 \\
766\end{array}$ & $\begin{array}{r}1671 \\
1822 \\
1761 \\
1785 \\
1475 \\
1058 \\
750 \\
660 \\
499 \\
446 \\
360 \\
390 \\
384 \\
434 \\
413 \\
445 \\
412 \\
489 \\
534\end{array}$ & $\begin{array}{l}742 \\
812 \\
790 \\
964 \\
940 \\
730 \\
567 \\
504 \\
432 \\
385 \\
332 \\
358 \\
353 \\
433 \\
368 \\
476 \\
455 \\
544 \\
613\end{array}$ & $\begin{array}{l}323 \\
358 \\
317 \\
449 \\
518 \\
456 \\
388 \\
350 \\
342 \\
303 \\
287 \\
313 \\
326 \\
404 \\
338 \\
455 \\
489 \\
552 \\
623\end{array}$ & $\begin{array}{l}137 \\
145 \\
117 \\
181 \\
252 \\
244 \\
243 \\
211 \\
247 \\
211 \\
215 \\
250 \\
263 \\
318 \\
279 \\
343 \\
387 \\
389 \\
461\end{array}$ & $\begin{array}{r}75 \\
79 \\
65 \\
91 \\
135 \\
156 \\
167 \\
142 \\
194 \\
173 \\
184 \\
224 \\
230 \\
243 \\
223 \\
261 \\
277 \\
253 \\
283\end{array}$ & $\begin{array}{l}31 \\
33 \\
27 \\
39 \\
56 \\
68 \\
81 \\
72 \\
98 \\
89 \\
103 \\
115 \\
106 \\
108 \\
108 \\
104 \\
109 \\
93 \\
96\end{array}$ & $\begin{array}{r}48 \\
55 \\
44 \\
56 \\
84 \\
117 \\
141 \\
124 \\
167 \\
171 \\
203 \\
187 \\
174 \\
152 \\
171 \\
144 \\
142 \\
105 \\
107\end{array}$ & $\begin{array}{l}25 \\
29 \\
23 \\
27 \\
38 \\
50 \\
54 \\
52 \\
68 \\
63 \\
68 \\
54 \\
53 \\
42 \\
48 \\
37 \\
38 \\
25 \\
31\end{array}$ & $\begin{array}{r}6.93 \\
8.27 \\
7.02 \\
6.22 \\
7.20 \\
8.00 \\
9.51 \\
6.58 \\
10.22 \\
7.29 \\
8.53 \\
7.47 \\
6.58 \\
6.84 \\
6.31 \\
5.42 \\
5.96 \\
4.44 \\
6.40\end{array}$ & $\begin{array}{l}1.51 \\
2.13 \\
1.42 \\
2.22 \\
2.67 \\
2.84 \\
3.20 \\
2.76 \\
3.02 \\
2.13 \\
2.31 \\
2.22 \\
2.31 \\
1.96 \\
1.60 \\
1.69 \\
2.04 \\
1.24 \\
2.49\end{array}$ & $\begin{array}{r}18059 \\
17880 \\
15665 \\
13937 \\
11213 \\
7975 \\
6224 \\
5294 \\
4363 \\
3994 \\
3674 \\
3723 \\
3822 \\
4123 \\
4595 \\
4363 \\
4157 \\
4525 \\
4825\end{array}$ & $\begin{array}{l}3.27 \\
3.19 \\
3.13 \\
3.13 \\
2.92 \\
2.69 \\
2.53 \\
2.54 \\
2.31 \\
2.36 \\
2.26 \\
2.28 \\
2.34 \\
2.38 \\
2.43 \\
2.45 \\
2.38 \\
2.58 \\
2.45\end{array}$ \\
\hline
\end{tabular}


Table B-9 - Remaining Number of Particles per ml for G=75 $\mathrm{s}^{-1}$ ( Mixing Tank)

\begin{tabular}{|c|c|c|c|c|c|c|c|c|c|c|c|c|c|c|}
\hline $\begin{array}{l}\text { Time } \\
\text { min. }\end{array}$ & 3.5 & 7.5 & 12.5 & $\begin{array}{l}\text { Avera } \\
17.5\end{array}$ & $\begin{array}{l}\text { ge } \overline{P z} \\
22.5\end{array}$ & $\begin{array}{l}\text { articl } \\
27.5\end{array}$ & $\begin{array}{c}\text { e } \$ 12 \\
32.5\end{array}$ & $\begin{array}{l}3 \mathrm{e}, \mathrm{M} \\
37.5\end{array}$ & $\begin{array}{l}\text { cron } \\
45\end{array}$ & $\begin{array}{l}18 \\
62.5\end{array}$ & 87.5 & 125 & Total & $\begin{array}{c}\text { Distribution } \\
\text { slope, } \beta\end{array}$ \\
\hline $\begin{array}{r}0.00 \\
0.75 \\
3.50 \\
6.00 \\
7.25 \\
10.75 \\
13.25 \\
16.25 \\
19.25 \\
21.75 \\
24.25 \\
30.00 \\
35.00 \\
39.50 \\
49.25 \\
59.50\end{array}$ & $\begin{array}{l}8671 \\
7939 \\
5981 \\
4064 \\
2831 \\
2074 \\
1756 \\
1421 \\
1425 \\
1330 \\
1359 \\
1481 \\
1467 \\
1353 \\
1466 \\
1456\end{array}$ & $\begin{array}{r}5406 \\
5019 \\
4071 \\
3005 \\
2039 \\
1454 \\
1221 \\
1008 \\
988 \\
898 \\
925 \\
1000 \\
954 \\
959 \\
1078 \\
1065\end{array}$ & $\begin{array}{r}1625 \\
1563 \\
1315 \\
1345 \\
988 \\
775 \\
638 \\
564 \\
560 \\
509 \\
540 \\
614 \\
589 \\
669 \\
824 \\
846\end{array}$ & $\begin{array}{l}702 \\
715 \\
552 \\
779 \\
673 \\
590 \\
501 \\
450 \\
505 \\
439 \\
491 \\
610 \\
575 \\
688 \\
873 \\
923\end{array}$ & $\begin{array}{l}319 \\
326 \\
228 \\
415 \\
418 \\
400 \\
384 \\
345 \\
409 \\
359 \\
417 \\
516 \\
509 \\
635 \\
747 \\
776\end{array}$ & $\begin{array}{r}123 \\
131 \\
92 \\
193 \\
222 \\
246 \\
227 \\
222 \\
280 \\
243 \\
282 \\
331 \\
330 \\
387 \\
403 \\
420\end{array}$ & $\begin{array}{r}67 \\
65 \\
51 \\
124 \\
139 \\
155 \\
161 \\
160 \\
203 \\
180 \\
203 \\
224 \\
206 \\
226 \\
204 \\
210\end{array}$ & $\begin{array}{r}29 \\
25 \\
23 \\
50 \\
59 \\
74 \\
76 \\
74 \\
101 \\
88 \\
91 \\
90 \\
83 \\
77 \\
66 \\
71\end{array}$ & $\begin{array}{r}49 \\
43 \\
37 \\
87 \\
115 \\
130 \\
143 \\
121 \\
150 \\
136 \\
137 \\
123 \\
106 \\
92 \\
75 \\
83\end{array}$ & $\begin{array}{l}23 \\
20 \\
20 \\
40 \\
45 \\
49 \\
55 \\
35 \\
45 \\
43 \\
43 \\
38 \\
32 \\
29 \\
23 \\
32\end{array}$ & $\begin{array}{l}5.24 \\
4.71 \\
5.33 \\
6.13 \\
8.18 \\
7.64 \\
7.02 \\
4.53 \\
7.38 \\
6.31 \\
5.87 \\
7.38 \\
5.16 \\
4.18 \\
4.98 \\
6.49\end{array}$ & $\begin{array}{l}0.62 \\
1.16 \\
0.89 \\
1.51 \\
1.42 \\
1.87 \\
1.51 \\
0.80 \\
1.96 \\
0.89 \\
1.16 \\
1.60 \\
1.78 \\
1.07 \\
1.42 \\
0.89\end{array}$ & $\begin{array}{r}17019 \\
15852 \\
12376 \\
10109 \\
7540 \\
5956 \\
5170 \\
4403 \\
4674 \\
4231 \\
4496 \\
5035 \\
4859 \\
5120 \\
5766 \\
5889\end{array}$ & $\begin{array}{l}3.25 \\
3.34 \\
3.28 \\
2.97 \\
2.79 \\
2.62 \\
2.57 \\
2.67 \\
2.45 \\
2.55 \\
2.56 \\
2.53 \\
2.55 \\
2.70 \\
2.71 \\
2.72\end{array}$ \\
\hline
\end{tabular}




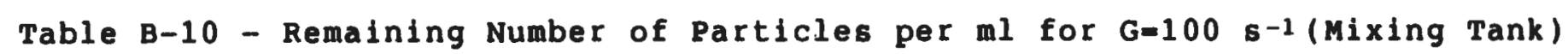

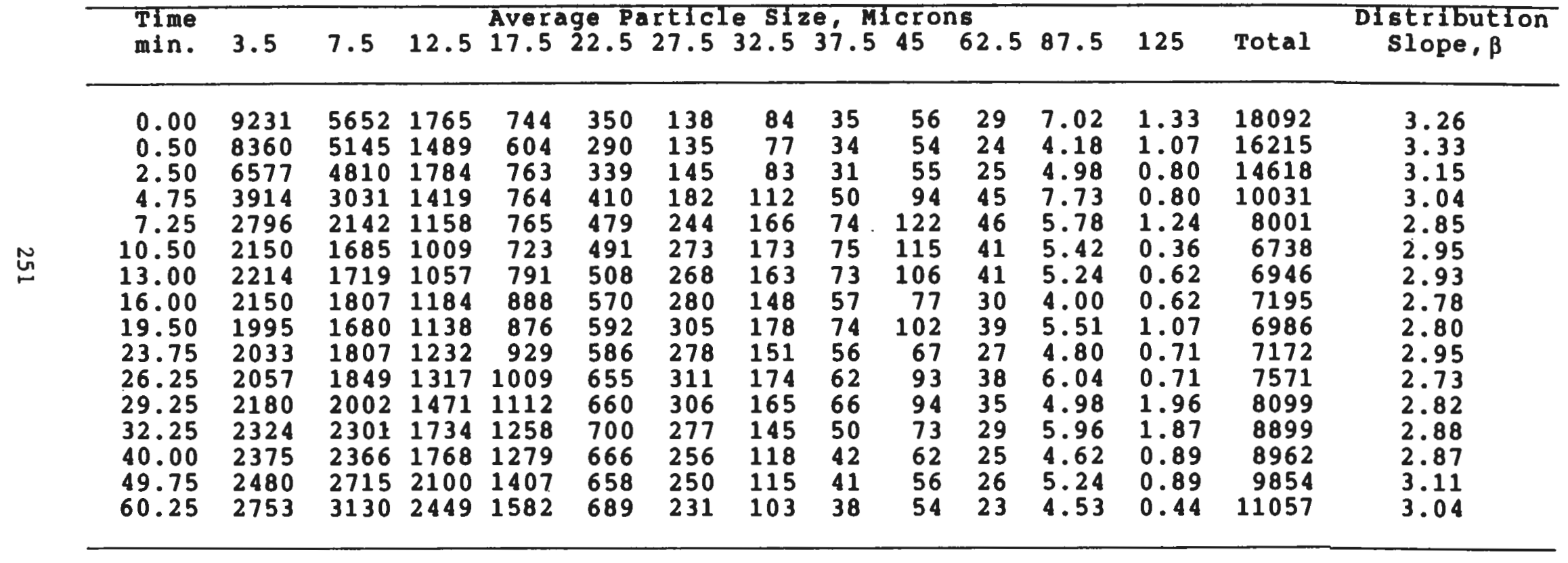




\section{APPENDIX C}

Pilot-Plant Data

- Data for Filter Number 1

- Data for Filter Number 2

- Data for Filter Number 3 
Table C-1 - Effluent Number of Particles per ml for Filter No. 1 (.35 mm)

\begin{tabular}{|c|c|c|c|c|c|c|c|c|c|c|c|c|}
\hline Time & & & Average & e Parti & le size & $\mathrm{micr}$ & ometer & & & & & \\
\hline (hrs) & 3.5 & 7.5 & 12.5 & 17.5 & 22.5 & 27.5 & 32.5 & 37.5 & 45.0 & 62.5 & 87.5 & 125 \\
\hline $\begin{array}{l}0.25 \\
1 \\
1.5 \\
2 \\
3 \\
4 \\
7 \\
8 \\
9 \\
15 \\
17 \\
19 \\
20 \\
22\end{array}$ & $\begin{array}{r}9.98 \\
7.77 \\
6.50 \\
5.11 \\
3.63 \\
4.70 \\
6.70 \\
10.87 \\
13.22 \\
23.08 \\
32.51 \\
147.78 \\
161.33 \\
263.63\end{array}$ & $\begin{array}{r}1.88 \\
1.48 \\
1.50 \\
1.26 \\
1.08 \\
1.11 \\
2.38 \\
3.99 \\
4.51 \\
14.23 \\
27.00 \\
111.60 \\
126.33 \\
209.26\end{array}$ & $\begin{array}{r}.13 \\
.26 \\
.28 \\
.12 \\
.15 \\
.09 \\
.24 \\
.20 \\
.33 \\
2.04 \\
4.67 \\
23.35 \\
30.08 \\
65.33\end{array}$ & $\begin{array}{r}.09 \\
.10 \\
.18 \\
.08 \\
.03 \\
.08 \\
.04 \\
.06 \\
.07 \\
.31 \\
.73 \\
3.58 \\
4.84 \\
17.26\end{array}$ & $\begin{array}{l}.04 \\
.08 \\
.07 \\
.06 \\
.03 \\
.06 \\
.03 \\
.07 \\
.06 \\
.12 \\
.03 \\
.43 \\
.68 \\
2.50\end{array}$ & $\begin{array}{l}0 \\
0 \\
.02 \\
0 \\
0 \\
.03 \\
0 \\
0 \\
0 \\
.02 \\
0 \\
.07 \\
.06 \\
.34\end{array}$ & $\begin{array}{l}0 \\
0 \\
0 \\
0 \\
0 \\
0 \\
0 \\
0 \\
0 \\
0 \\
0 \\
.03 \\
.04 \\
0\end{array}$ & $\begin{array}{l}0 \\
0 \\
0 \\
0 \\
0 \\
0 \\
0 \\
0 \\
0 \\
0 \\
0 \\
0 \\
03 \\
0\end{array}$ & $\begin{array}{l}0 \\
0 \\
0 \\
0 \\
0 \\
0 \\
0 \\
0 \\
0 \\
0 \\
0 \\
0 \\
0 \\
0\end{array}$ & $\begin{array}{l}0 \\
0 \\
0 \\
0 \\
0 \\
0 \\
0 \\
0 \\
0 \\
0 \\
0 \\
0 \\
0 \\
0\end{array}$ & $\begin{array}{l}0 \\
0 \\
0 \\
0 \\
0 \\
0 \\
0 \\
0 \\
0 \\
0 \\
0 \\
0 \\
0 \\
0\end{array}$ & $\begin{array}{l}0 \\
0 \\
0 \\
0 \\
0 \\
0 \\
0 \\
0 \\
0 \\
0 \\
0 \\
0 \\
0 \\
0\end{array}$ \\
\hline
\end{tabular}

Note: Alum dose $=0.0 \mathrm{mg} / \mathrm{L}, G=50 \mathrm{~s}^{-1}, t=30$ minutes 
Table C-2 - Effluent Number of Particles per ml for Filter No.1(.35 mm)

\begin{tabular}{|c|c|c|c|c|c|c|c|c|c|c|c|c|}
\hline Time & & & Averag & e part & le siz & $\therefore \mathrm{mic}$ & romete & & & & & \\
\hline (hrs) & 3.5 & 7.5 & 12.5 & 17.5 & 22.5 & 27.5 & 32.5 & 37.5 & 45.0 & 62.5 & 87.5 & 125 \\
\hline $\begin{array}{l}0.25 \\
1 \\
2 \\
4 \\
6 \\
9 \\
12 \\
16 \\
22.5\end{array}$ & $\begin{array}{l}9.41 \\
5.88 \\
5.28 \\
4.22 \\
3.49 \\
6.25 \\
6.45 \\
6.74 \\
7.74\end{array}$ & $\begin{array}{r}1.26 \\
.63 \\
.62 \\
.56 \\
.35 \\
.80 \\
.79 \\
.82 \\
1.02\end{array}$ & $\begin{array}{l}.08 \\
.04 \\
.04 \\
.05 \\
.02 \\
.09 \\
.05 \\
.06 \\
.08\end{array}$ & $\begin{array}{l}.02 \\
.02 \\
.01 \\
.03 \\
.02 \\
.05 \\
.03 \\
.02 \\
.01\end{array}$ & $\begin{array}{l}.01 \\
.02 \\
0 \\
.03 \\
0 \\
.01 \\
0 \\
0 \\
0\end{array}$ & $\begin{array}{l}0 \\
0 \\
0 \\
01 \\
0 \\
01 \\
0 \\
0 \\
0 \\
0\end{array}$ & $\begin{array}{l}0 \\
0 \\
0 \\
01 \\
0 \\
01 \\
0 \\
0 \\
0\end{array}$ & $\begin{array}{l}0 \\
0 \\
0 \\
0 \\
0 \\
0 \\
0 \\
0 \\
0\end{array}$ & $\begin{array}{l}0 \\
0 \\
0 \\
0 \\
0 \\
0 \\
0 \\
0 \\
0\end{array}$ & $\begin{array}{l}0 \\
0 \\
0 \\
0 \\
0 \\
0 \\
0 \\
0 \\
0\end{array}$ & $\begin{array}{l}0 \\
0 \\
0 \\
0 \\
0 \\
0 \\
0 \\
0 \\
0\end{array}$ & $\begin{array}{l}0 \\
0 \\
0 \\
0 \\
0 \\
0 \\
0 \\
0 \\
0\end{array}$ \\
\hline
\end{tabular}

NOTE: Alum dose $=2.0 \mathrm{mg} / \mathrm{L}, G=50 \mathrm{~s}-1, t=30$ minutes 
Table C-3 - Effluent Number of Particles per ml for Filter No. 1 (.35 mm)

\begin{tabular}{|c|c|c|c|c|c|c|c|c|c|c|c|c|}
\hline Time & & & verage & Part: & cle $\mathrm{Siz}$ & ze, mi & cromet & & & & & \\
\hline$(h r s)$ & 3.5 & 7.5 & 12.5 & 17.5 & 22.5 & 27.5 & 32.5 & 37.5 & 45.0 & 62.5 & 87.5 & 125 \\
\hline $\begin{array}{l}0.25 \\
1 \\
2 \\
3 \\
4 \\
5 \\
6 \\
7 \\
8 \\
9 \\
11\end{array}$ & $\begin{array}{l}2.53 \\
1.36 \\
1.36 \\
0.92 \\
1.37 \\
1.46 \\
1.34 \\
1.43 \\
2.56 \\
3.09 \\
3.59\end{array}$ & $\begin{array}{r}.60 \\
.20 \\
.21 \\
.49 \\
.30 \\
.17 \\
.66 \\
.41 \\
.73 \\
.85 \\
1.14\end{array}$ & $\begin{array}{l}.17 \\
.04 \\
.07 \\
.17 \\
.09 \\
.13 \\
.08 \\
.10 \\
.06 \\
.13 \\
.13\end{array}$ & $\begin{array}{l}.16 \\
0 \\
.04 \\
.06 \\
.03 \\
.04 \\
.03 \\
.21 \\
.06 \\
.06 \\
.04\end{array}$ & $\begin{array}{l}.04 \\
0 \\
.04 \\
.03 \\
0 \\
0 \\
.01 \\
.09 \\
0 \\
.02 \\
.03\end{array}$ & $\begin{array}{l}.02 \\
0 \\
0 \\
.04 \\
0 \\
0 \\
0 \\
.03 \\
0 \\
0 \\
0\end{array}$ & $\begin{array}{l}0 \\
0 \\
0 \\
0 \\
0 \\
0 \\
0 \\
0 \\
0 \\
0 \\
0\end{array}$ & $\begin{array}{l}0 \\
0 \\
0 \\
0 \\
0 \\
0 \\
0 \\
0 \\
0 \\
0 \\
0\end{array}$ & $\begin{array}{l}0 \\
0 \\
0 \\
0 \\
0 \\
0 \\
0 \\
0 \\
0 \\
0 \\
0\end{array}$ & $\begin{array}{l}0 \\
0 \\
0 \\
0 \\
0 \\
0 \\
0 \\
0 \\
0 \\
0 \\
0\end{array}$ & $\begin{array}{l}0 \\
0 \\
0 \\
0 \\
0 \\
0 \\
0 \\
0 \\
0 \\
0 \\
0\end{array}$ & $\begin{array}{l}0 \\
0 \\
0 \\
0 \\
0 \\
0 \\
0 \\
0 \\
0 \\
0 \\
0\end{array}$ \\
\hline
\end{tabular}

Note: Alum dose=8.0 $\mathrm{mg} / \mathrm{L}, G=50 \mathrm{~s}^{-1}, t=30$ minutes 
Table C-4 - Effluent Number of Particles per ml fór Filter No. 1 (.35 mm)

\begin{tabular}{|c|c|c|c|c|c|c|c|c|c|c|c|c|}
\hline Time & & & Average & Parti & le siz & e, mic & cromet & & & & & \\
\hline (hrs) & 3.5 & 7.5 & 12.5 & 17.5 & 22.5 & 27.5 & 32.5 & 37.5 & 45 & 62.5 & 87.5 & 125 \\
\hline $\begin{array}{l}0.25 \\
1 \\
2 \\
3 \\
3.5 \\
4\end{array}$ & $\begin{array}{l}1.54 \\
1.14 \\
0.98 \\
0.76 \\
1.69 \\
1.97\end{array}$ & $\begin{array}{l}.29 \\
.31 \\
.19 \\
.22 \\
.40 \\
.77\end{array}$ & $\begin{array}{l}.06 \\
.03 \\
.03 \\
.06 \\
.04 \\
.30\end{array}$ & $\begin{array}{l}.08 \\
.03 \\
0 \\
.07 \\
.16 \\
.09\end{array}$ & $\begin{array}{l}.02 \\
0 \\
0 \\
.02 \\
.02 \\
.02\end{array}$ & $\begin{array}{l}.01 \\
0 \\
0 \\
0 \\
0 \\
0\end{array}$ & $\begin{array}{l}0 \\
0 \\
0 \\
0 \\
0 \\
0\end{array}$ & $\begin{array}{l}0 \\
0 \\
0 \\
0 \\
0 \\
0\end{array}$ & $\begin{array}{l}0 \\
0 \\
0 \\
0 \\
0 \\
0\end{array}$ & $\begin{array}{l}0 \\
0 \\
0 \\
0 \\
0 \\
0\end{array}$ & $\begin{array}{l}0 \\
0 \\
0 \\
0 \\
0 \\
0\end{array}$ & $\begin{array}{l}0 \\
0 \\
0 \\
0 \\
0 \\
0\end{array}$ \\
\hline
\end{tabular}

Note: Alum dose $=16.0 \mathrm{mg} / \mathrm{L}, G=50 \mathrm{~s}^{-1}, t=30$ minutes 
Table C-5 - Effluent Number of Particles per ml for Filter No. 1 (.35 mm)

\begin{tabular}{|c|c|c|c|c|c|c|c|c|c|c|c|c|}
\hline & & & Average & Part 1 & cle si & ze, $m$ : & cromet & & & & & \\
\hline (hrs) & 3.5 & 7.5 & 12.5 & 17.5 & 22.5 & 27.5 & 32.5 & 37.5 & 45.0 & 62.5 & 87.5 & 125 \\
\hline $\begin{array}{l}0.25 \\
1 \\
2 \\
5 \\
6 \\
7 \\
8 \\
9\end{array}$ & $\begin{array}{l}1.04 \\
1.02 \\
0.98 \\
0.94 \\
0.83 \\
1.40 \\
1.78 \\
2.27\end{array}$ & $\begin{array}{l}.12 \\
.09 \\
.11 \\
.10 \\
.09 \\
.15 \\
.22 \\
.28\end{array}$ & $\begin{array}{c}.01 \\
.01 \\
0 \\
.01 \\
0 \\
.01 \\
.01 \\
.03\end{array}$ & $\begin{array}{l}0 \\
0 \\
0 \\
0 \\
0 \\
0 \\
0 \\
.01\end{array}$ & $\begin{array}{l}0 \\
0 \\
0 \\
0 \\
0 \\
0 \\
0 \\
.01\end{array}$ & $\begin{array}{l}0 \\
0 \\
0 \\
0 \\
0 \\
0 \\
0 \\
0\end{array}$ & $\begin{array}{l}0 \\
0 \\
0 \\
0 \\
0 \\
0 \\
0 \\
0\end{array}$ & $\begin{array}{l}0 \\
0 \\
0 \\
0 \\
0 \\
0 \\
0 \\
0\end{array}$ & $\begin{array}{l}0 \\
0 \\
0 \\
0 \\
0 \\
0 \\
0 \\
0\end{array}$ & $\begin{array}{l}0 \\
0 \\
0 \\
0 \\
0 \\
0 \\
0 \\
0\end{array}$ & $\begin{array}{l}0 \\
0 \\
0 \\
0 \\
0 \\
0 \\
0 \\
0\end{array}$ & $\begin{array}{l}0 \\
0 \\
0 \\
0 \\
0 \\
0 \\
0 \\
0\end{array}$ \\
\hline
\end{tabular}

Note: Alum dose-8.0 $\mathrm{mg} / \mathrm{L}, G=25 \mathrm{~s}^{-1}, t=30 \mathrm{minutes}$ 
Table C-6 - Effluent Number of Particles per ml for Filter No. 1 (.35 mm)

\begin{tabular}{|c|c|c|c|c|c|c|c|c|c|c|c|c|}
\hline Time & & & Average & Part: & le 512 & e, mic & romete & & & & & \\
\hline$(h \times s)$ & 3.5 & 7.5 & 12.5 & 17.5 & 22.5 & 27.5 & 32.5 & 37.5 & 45.0 & 62.5 & 87.5 & 125 \\
\hline $\begin{array}{l}0.25 \\
1 \\
2 \\
3 \\
4 \\
6 \\
7 \\
8 \\
9 \\
11 \\
12 \\
12.75\end{array}$ & $\begin{array}{l}3.47 \\
1.87 \\
1.83 \\
1.66 \\
1.83 \\
2.05 \\
2.94 \\
3.22 \\
3.54 \\
3.72 \\
3.89 \\
3.96\end{array}$ & $\begin{array}{l}.43 \\
.24 \\
.22 \\
.20 \\
.19 \\
.26 \\
.43 \\
.46 \\
.48 \\
.52 \\
.55 \\
.57\end{array}$ & $\begin{array}{l}.04 \\
.03 \\
.01 \\
.02 \\
.02 \\
.01 \\
.06 \\
.04 \\
.05 \\
.05 \\
.04 \\
.07\end{array}$ & $\begin{array}{l}.01 \\
.02 \\
0 \\
.01 \\
.01 \\
.01 \\
.03 \\
.01 \\
.01 \\
.01 \\
.01 \\
.04\end{array}$ & $\begin{array}{l}.01 \\
.01 \\
0 \\
.01 \\
.01 \\
0 \\
.01 \\
0 \\
0 \\
0 \\
0 \\
.02\end{array}$ & $\begin{array}{l}0 \\
0 \\
0 \\
0 \\
.01 \\
0 \\
0 \\
0 \\
0 \\
0 \\
0 \\
01\end{array}$ & $\begin{array}{l}0 \\
0 \\
0 \\
0 \\
0 \\
0 \\
0 \\
0 \\
0 \\
0 \\
0 \\
.01\end{array}$ & $\begin{array}{l}0 \\
0 \\
0 \\
0 \\
0 \\
0 \\
0 \\
0 \\
0 \\
0 \\
0 \\
01\end{array}$ & $\begin{array}{l}0 \\
0 \\
0 \\
0 \\
0 \\
0 \\
0 \\
0 \\
0 \\
0 \\
0 \\
0\end{array}$ & $\begin{array}{l}0 \\
0 \\
0 \\
0 \\
0 \\
0 \\
0 \\
0 \\
0 \\
0 \\
0 \\
0\end{array}$ & $\begin{array}{l}0 \\
0 \\
0 \\
0 \\
0 \\
0 \\
0 \\
0 \\
0 \\
0 \\
0 \\
0\end{array}$ & $\begin{array}{l}0 \\
0 \\
0 \\
0 \\
0 \\
0 \\
0 \\
0 \\
0 \\
0 \\
0 \\
0\end{array}$ \\
\hline
\end{tabular}

Note: Alum dose=8.0 $\mathrm{mg} / \mathrm{L}, \mathrm{G}=100 \mathrm{~s}^{-1}, t-30 \mathrm{minutes}$ 
Table C-7 - Effluent number of Particles per ml for Filter No. 1 (.35 mm)

\begin{tabular}{|c|c|c|c|c|c|c|c|c|c|c|c|c|}
\hline Time & & & Average & Part & Le siz & $e, m i c$ & comete & & & & & \\
\hline (hrs) & 3.5 & 7.5 & 12.5 & 17.5 & 22.5 & 27.5 & 32.5 & 37.5 & 45.0 & 62.5 & 87.5 & 125 \\
\hline $\begin{array}{l}0.25 \\
1 \\
2 \\
3 \\
4 \\
4.75\end{array}$ & $\begin{array}{l}9.44 \\
3.54 \\
3.77 \\
4.53 \\
3.77 \\
4.57\end{array}$ & $\begin{array}{l}2.23 \\
0.74 \\
1.52 \\
0.83 \\
0.73 \\
1.09\end{array}$ & $\begin{array}{l}.30 \\
.15 \\
.40 \\
.10 \\
.07 \\
.21\end{array}$ & $\begin{array}{l}.04 \\
.07 \\
.27 \\
.01 \\
.06 \\
.10\end{array}$ & $\begin{array}{l}.04 \\
0 \\
.07 \\
0 \\
0 \\
0\end{array}$ & $\begin{array}{l}.02 \\
0 \\
.03 \\
0 \\
0 \\
0\end{array}$ & $\begin{array}{l}0 \\
0 \\
.02 \\
0 \\
0 \\
0\end{array}$ & $\begin{array}{l}0 \\
0 \\
0 \\
0 \\
0 \\
0\end{array}$ & $\begin{array}{l}0 \\
0 \\
0 \\
0 \\
0 \\
0\end{array}$ & $\begin{array}{l}0 \\
0 \\
0 \\
0 \\
0 \\
0\end{array}$ & $\begin{array}{l}0 \\
0 \\
0 \\
0 \\
0 \\
0\end{array}$ & $\begin{array}{l}0 \\
0 \\
0 \\
0 \\
0 \\
0\end{array}$ \\
\hline
\end{tabular}

Note: Alum dose $=8.0 \mathrm{mg} / \mathrm{L}, G=50 \mathrm{~s}^{-1}, t=15$ minutes 
Table C-8 - Effluent Number of Particles per ml for Filter No. 1 (.35 mm)

\begin{tabular}{|c|c|c|c|c|c|c|c|c|c|c|c|c|}
\hline & & & Average & Parti & le Siz & e, mic & omete & & & & & \\
\hline (hrs) & 3.5 & 7.5 & 12.5 & 17.5 & 22.5 & 27.5 & 32.5 & 37.5 & 45.0 & 62.5 & 87.5 & 125 \\
\hline $\begin{array}{l}0.25 \\
1 \\
2 \\
3 \\
5 \\
7 \\
9 \\
12\end{array}$ & $\begin{array}{l}3.39 \\
1.23 \\
0.83 \\
1.10 \\
1.58 . \\
2.24 \\
2.80 \\
3.18\end{array}$ & $\begin{array}{l}1.02 \\
0.18 \\
0.23 \\
0.28 \\
0.48 \\
0.84 \\
0.58 \\
0.83\end{array}$ & $\begin{array}{l}.13 \\
.03 \\
.11 \\
.10 \\
.09 \\
.08 \\
.11 \\
.11\end{array}$ & $\begin{array}{l}.07 \\
.05 \\
.02 \\
.03 \\
.02 \\
.01 \\
.06 \\
.18\end{array}$ & $\begin{array}{l}.02 \\
0 \\
0 \\
0 \\
0 \\
01 \\
0 \\
0\end{array}$ & $\begin{array}{l}.03 \\
0 \\
0 \\
0 \\
0 \\
.01 \\
0 \\
0\end{array}$ & $\begin{array}{l}0 \\
0 \\
0 \\
0 \\
0 \\
01 \\
0 \\
0\end{array}$ & $\begin{array}{l}0 \\
0 \\
0 \\
0 \\
0 \\
0 \\
0 \\
0\end{array}$ & $\begin{array}{l}0 \\
0 \\
0 \\
0 \\
0 \\
0 \\
0 \\
0\end{array}$ & $\begin{array}{l}0 \\
0 \\
0 \\
0 \\
0 \\
0 \\
0 \\
0\end{array}$ & $\begin{array}{l}0 \\
0 \\
0 \\
0 \\
0 \\
0 \\
0 \\
0\end{array}$ & $\begin{array}{l}0 \\
0 \\
0 \\
0 \\
0 \\
0 \\
0 \\
0\end{array}$ \\
\hline & & & & & & & & & & & & \\
\hline
\end{tabular}

Note: Alum dose-8.0 mg/L, G=50 $\mathrm{s}^{-1}, t=45$ minutes 
Table C-9 - Effluent Number of Particles per ml for Filter No. $2(.50 \mathrm{~mm})$

\begin{tabular}{|c|c|c|c|c|c|c|c|c|c|c|c|c|}
\hline & & & Average & Particle & size, & micr & omete & & & & & \\
\hline (hrs) & 3.5 & 7.5 & 12.5 & 17.5 & 22.5 & 27.5 & 32.5 & 37.5 & 45.0 & 62.5 & 87.5 & 125 \\
\hline $\begin{array}{l}0.25 \\
1 \\
2 \\
3 \\
4 \\
7 \\
9 \\
15 \\
17 \\
19 \\
20 \\
22 \\
25 \\
26\end{array}$ & $\begin{array}{r}115 \\
87 \\
82 \\
53 \\
61 \\
95 \\
101 \\
160 \\
195 \\
411 \\
447 \\
474 \\
789 \\
875\end{array}$ & $\begin{array}{r}46 \\
25 \\
26 \\
16 \\
21 \\
36 \\
66 \\
120 \\
160 \\
341 \\
383 \\
437 \\
700 \\
812\end{array}$ & $\begin{array}{c}4.1 \\
3.1 \\
3.6 \\
3.4 \\
4.8 \\
11 \\
23 \\
51 \\
75 \\
148 \\
153 \\
183 \\
257 \\
316\end{array}$ & $\begin{array}{c}0.61 \\
0.41 \\
0.71 \\
0.61 \\
1.2 \\
2.9 \\
7.4 \\
23 \\
38 \\
65 \\
63 \\
79 \\
104 \\
122\end{array}$ & $\begin{array}{l}0.25 \\
0.12 \\
0.10 \\
0.12 \\
0.17 \\
0.63 \\
1.7 \\
8.3 \\
14 \\
23 \\
22 \\
28 \\
33 \\
41\end{array}$ & $\begin{array}{c}0 \\
0.08 \\
0 \\
0.04 \\
0.02 \\
0.04 \\
0.29 \\
2.1 \\
4.1 \\
6.0 \\
6.4 \\
7.4 \\
9.0 \\
11\end{array}$ & $\begin{array}{l}0 \\
0 \\
0 \\
.03 \\
.01 \\
0 \\
.06 \\
.69 \\
1.2 \\
2.3 \\
2.2 \\
2.4 \\
2.6 \\
3.9\end{array}$ & $\begin{array}{l}0 \\
0 \\
0 \\
.02 \\
0 \\
0 \\
0 \\
.20 \\
.32 \\
.38 \\
.46 \\
.66 \\
.74 \\
.86\end{array}$ & $\begin{array}{l}0 \\
0 \\
0 \\
0 \\
0 \\
0 \\
0 \\
.07 \\
.30 \\
.32 \\
.29 \\
.41 \\
.51 \\
.58\end{array}$ & $\begin{array}{l}0 \\
0 \\
0 \\
0 \\
0 \\
0 \\
0 \\
.01 \\
.01 \\
.02 \\
.01 \\
.02 \\
.04 \\
.03\end{array}$ & $\begin{array}{l}0 \\
0 \\
0 \\
0 \\
0 \\
0 \\
0 \\
0 \\
0 \\
0 \\
0 \\
0 \\
.01 \\
.01\end{array}$ & $\begin{array}{l}0 \\
0 \\
0 \\
0 \\
0 \\
0 \\
0 \\
0 \\
0 \\
0 \\
0 \\
0 \\
0 \\
0\end{array}$ \\
\hline & & & & & & & & & & & & \\
\hline
\end{tabular}

Note: Alum dose $=0.0 \mathrm{mg} / \mathrm{L}, G=50 \mathrm{~s}^{-1}, t=30$ minutes 
Table C-10 - Effluent Number of Particles per ml for Filter No. 2 (.50 mm)

\begin{tabular}{|c|c|c|c|c|c|c|c|c|c|c|c|c|}
\hline \multirow{2}{*}{$\begin{array}{l}\text { Time } \\
\text { (hrs) }\end{array}$} & \multirow[b]{2}{*}{3.5} & \multicolumn{2}{|r|}{ Average } & \multirow{2}{*}{$\begin{array}{r}\text { Particle } \\
17.5\end{array}$} & \multirow{2}{*}{$\begin{array}{l}\text { size, } \\
22.5\end{array}$} & \multicolumn{3}{|c|}{ micrometer } & \multirow[b]{2}{*}{45.0} & \multirow[b]{2}{*}{62.5} & \multirow[b]{2}{*}{87.5} & \multirow[b]{2}{*}{125} \\
\hline & & 7.5 & 12.5 & & & ; $\quad 27.5$ & 32.5 & 37.5 & & & & \\
\hline $\begin{array}{l}0.25 \\
1 \\
2 \\
5 \\
8 \\
14 \\
23 \\
28 \\
29 \\
31 \\
32 \\
37 \\
48 \\
51 \\
57 \\
60 \\
61 \\
71\end{array}$ & $\begin{array}{r}50 \\
45 \\
42 \\
31 \\
33 \\
15 \\
25 \\
39 \\
35 \\
41 \\
41 \\
50 \\
65 \\
81 \\
122 \\
115 \\
176 \\
247\end{array}$ & $\begin{array}{r}9.0 \\
8.1 \\
7.0 \\
5.9 \\
4.6 \\
1.4 \\
11 \\
12 \\
14 \\
23 \\
22 \\
23 \\
54 \\
64 \\
104 \\
118 \\
182 \\
292\end{array}$ & $\begin{array}{l}0.77 \\
0.73 \\
0.68 \\
1.1 \\
0.32 \\
0.10 \\
5.5 \\
3.1 \\
6.4 \\
12 \\
12 \\
14 \\
35 \\
41 \\
68 \\
79 \\
126 \\
192\end{array}$ & $\begin{array}{l}0.16 \\
0.14 \\
0.16 \\
0.29 \\
0.04 \\
0.01 \\
3.4 \\
0.86 \\
3.9 \\
6.8 \\
8.2 \\
8.4 \\
26 \\
28 \\
47 \\
54 \\
88 \\
121\end{array}$ & $\begin{array}{l}0.06 \\
0.04 \\
0.06 \\
0.11 \\
0.01 \\
0.01 \\
1.7 \\
0.23 \\
2.4 \\
3.4 \\
5.0 \\
5.0 \\
17 \\
16 \\
28 \\
33 \\
55 \\
68\end{array}$ & $\begin{array}{c}0.02 \\
0.02 \\
0.02 \\
0.04 \\
0 \\
0 \\
0.60 \\
0.03 \\
0.89 \\
1.2 \\
2.0 \\
2.4 \\
8.8 \\
7.4 \\
13 \\
15 \\
28 \\
32\end{array}$ & $\begin{array}{c}0.01 \\
0.01 \\
0 \\
0 \\
0 \\
0 \\
0.19 \\
0 \\
0.34 \\
0.56 \\
0.91 \\
1.1 \\
4.5 \\
3.9 \\
6.9 \\
8.3 \\
16 \\
18\end{array}$ & $\begin{array}{c}0 \\
0 \\
0 \\
0 \\
0 \\
0 \\
0.09 \\
0 \\
0.10 \\
0.15 \\
0.30 \\
0.33 \\
1.7 \\
1.4 \\
2.6 \\
3.1 \\
6.5 \\
7.1\end{array}$ & $\begin{array}{c}0 \\
0 \\
0 \\
0 \\
0 \\
0 \\
0.06 \\
0 \\
0.10 \\
0.13 \\
0.21 \\
0.31 \\
2.3 \\
1.9 \\
3.6 \\
4.3 \\
10 \\
11\end{array}$ & $\begin{array}{l}0 \\
0 \\
0 \\
0 \\
0 \\
0 \\
0.01 \\
0 \\
0 \\
0.01 \\
3 \\
0.01 \\
0.03 \\
0.05 \\
0.42 \\
0.37 \\
0.80 \\
1.1 \\
2.9 \\
3.5\end{array}$ & $\begin{array}{l}0 \\
0 \\
0 \\
0 \\
0 \\
0 \\
0 \\
0 \\
0 \\
0 \\
0 \\
0 \\
.01 \\
0 \\
.03 \\
.02 \\
.05 \\
.12\end{array}$ & $\begin{array}{l}0 \\
0 \\
0 \\
0 \\
0 \\
0 \\
0 \\
0 \\
0 \\
0 \\
0 \\
0 \\
0 \\
0 \\
0 \\
0 \\
0 \\
0\end{array}$ \\
\hline
\end{tabular}

Note: Alum dose $=2.0 \mathrm{mg} / \mathrm{L}, G=50 \mathrm{~s}^{-1}, \mathrm{t}=30 \mathrm{minutes}$ 
Table C-11 - Effluent Number of Particles per ml for Filter No. 2 (.50 mm)

\begin{tabular}{|c|c|c|c|c|c|c|c|c|c|c|c|c|}
\hline \multirow{2}{*}{$\begin{array}{l}\text { Time } \\
\text { (hrs) }\end{array}$} & \multirow[b]{2}{*}{3.5} & \multicolumn{3}{|c|}{ Average Particle } & \multicolumn{3}{|c|}{ size, micrometer } & \multirow{2}{*}{37.5} & \multirow[b]{2}{*}{45.0} & \multirow[b]{2}{*}{62.5} & \multirow[b]{2}{*}{87.5} & \multirow[b]{2}{*}{125} \\
\hline & & 7.5 & 12.5 & 17.5 & 22.5 & 27.5 & 32.5 & & & & & \\
\hline $\begin{array}{l}0.25 \\
1 \\
3 \\
4 \\
5 \\
8 \\
11 \\
13 \\
14 \\
15 \\
28 \\
32\end{array}$ & $\begin{array}{r}2.1 \\
2.2 \\
1.8 \\
1.6 \\
2.1 \\
1.9 \\
2.0 \\
2.4 \\
2.2 \\
2.4 \\
8.8 \\
13\end{array}$ & $\begin{array}{l}0.53 \\
0.41 \\
0.25 \\
0.20 \\
0.30 \\
0.35 \\
0.52 \\
0.67 \\
0.80 \\
0.71 \\
5.3 \\
8.8\end{array}$ & $\begin{array}{l}0.08 \\
0.06 \\
0.04 \\
0.03 \\
0.05 \\
0.08 \\
0.21 \\
0.18 \\
0.35 \\
0.27 \\
3.7 \\
5.6\end{array}$ & $\begin{array}{l}0.02 \\
0.03 \\
0.01 \\
0.02 \\
0.03 \\
0.05 \\
0.12 \\
0.11 \\
0.26 \\
0.24 \\
3.5 \\
3.9\end{array}$ & $\begin{array}{c}0 \\
0.01 \\
0 \\
0.01 \\
0.03 \\
0.03 \\
0.05 \\
0.06 \\
0.18 \\
0.17 \\
2.7 \\
1.9\end{array}$ & $\begin{array}{c}0 \\
0.01 \\
0 \\
0 \\
0.01 \\
0.01 \\
0.01 \\
0.01 \\
0.06 \\
0.07 \\
1.4 \\
0.67\end{array}$ & $\begin{array}{l}0 \\
.01 \\
0 \\
0 \\
0 \\
0 \\
.01 \\
0 \\
.03 \\
.03 \\
.87 \\
.28\end{array}$ & $\begin{array}{l}0 \\
0 \\
0 \\
0 \\
0 \\
0 \\
0 \\
0 \\
.01 \\
.01 \\
.33 \\
.06\end{array}$ & $\begin{array}{l}0 \\
.01 \\
0 \\
0 \\
0 \\
0 \\
0 \\
0 \\
0 \\
0 \\
.39 \\
.04\end{array}$ & $\begin{array}{l}0 \\
.01 \\
0 \\
0 \\
0 \\
0 \\
0 \\
0 \\
0 \\
0 \\
.09 \\
0\end{array}$ & $\begin{array}{l}0 \\
0 \\
0 \\
0 \\
0 \\
0 \\
0 \\
0 \\
0 \\
0 \\
0 \\
0\end{array}$ & $\begin{array}{l}0 \\
0 \\
0 \\
0 \\
0 \\
0 \\
0 \\
0 \\
0 \\
0 \\
0 \\
0\end{array}$ \\
\hline
\end{tabular}

Note: Alum dose $=8.0 \mathrm{mg} / \mathrm{L}, G=50 \mathrm{~s}^{-1}, t=30$ minutes 
Table C-12 - Effluent Number of Particles per ml for Filter No. 2 (.50 mm)

\begin{tabular}{|c|c|c|c|c|c|c|c|c|c|c|c|c|}
\hline Time & & & Average & Parti & le size & $\operatorname{mic}$ & ometer & & & & & \\
\hline (hrs) & 3.5 & 7.5 & 12.5 & 17.5 & 22.5 & 27.5 & 32.5 & 37.5 & 45.0 & 62.5 & 87.5 & 125 \\
\hline $\begin{array}{l}0.25 \\
1 \\
2 \\
3 \\
6 \\
7 \\
8 \\
18 \\
19 \\
20 \\
21 \\
22\end{array}$ & $\begin{array}{l}1.5 \\
0.87 \\
0.46 \\
1.5 \\
9.2 \\
11 \\
16 \\
14 \\
18 \\
41 \\
72 \\
90\end{array}$ & $\begin{array}{l}0.25 \\
0.21 \\
0.12 \\
0.48 \\
4.3 \\
4.7 \\
6.7 \\
6.6 \\
7.8 \\
19 \\
35 \\
62\end{array}$ & $\begin{array}{l}0.04 \\
0.03 \\
0.02 \\
0.10 \\
1.2 \\
1.1 \\
1.7 \\
2.7 \\
3.2 \\
8.9 \\
19 \\
32\end{array}$ & $\begin{array}{l}0.01 \\
0.02 \\
0.01 \\
0.04 \\
0.64 \\
0.44 \\
0.77 \\
2.4 \\
2.8 \\
7.4 \\
16 \\
23\end{array}$ & $\begin{array}{l}0.01 \\
0.01 \\
0.01 \\
0.02 \\
0.33 \\
0.26 \\
0.56 \\
2.0 \\
2.2 \\
5.1 \\
11 \\
17\end{array}$ & $\begin{array}{l}\quad 0 \\
0.01 \\
0 \\
0.01 \\
0.16 \\
0.09 \\
0.35 \\
1.2 \\
1.3 \\
2.5 \\
5.6 \\
9.7\end{array}$ & $\begin{array}{l}0 \\
0 \\
0 \\
0 \\
0.05 \\
0.07 \\
0.22 \\
0.67 \\
0.75 \\
1.2 \\
3.1 \\
6.2\end{array}$ & $\begin{array}{c}0 \\
0 \\
0 \\
0 \\
0.01 \\
0.02 \\
0.08 \\
0.25 \\
0.29 \\
0.33 \\
1.2 \\
2.7\end{array}$ & $\begin{array}{c}0 \\
0 \\
0 \\
0 \\
0.01 \\
0.02 \\
0.13 \\
0.33 \\
0.40 \\
0.61 \\
1.3 \\
4.1\end{array}$ & $\begin{array}{c}0 \\
0 \\
0 \\
0 \\
0 \\
0 \\
0.03 \\
0.08 \\
0.11 \\
0.07 \\
0.25 \\
1.1\end{array}$ & $\begin{array}{l}0 \\
0 \\
0 \\
0 \\
0 \\
0 \\
0 \\
0 \\
0 \\
0 \\
.01 \\
.01\end{array}$ & $\begin{array}{l}0 \\
0 \\
0 \\
0 \\
0 \\
0 \\
0 \\
0 \\
0 \\
0 \\
0 \\
0\end{array}$ \\
\hline
\end{tabular}

Note: Alum dose $=16.0 \mathrm{mg} / \mathrm{L}, G=50 \mathrm{~s}^{-1}, t=30$ minutes 
Table C-13 - Effluent Number of Particles per ml for Filter No. 2 (.50 mm)

\begin{tabular}{|c|c|c|c|c|c|c|c|c|c|c|c|c|}
\hline Time & & & Average & Partic & e size, & - mict & ometer & & & & & \\
\hline (hrs) & 3.5 & 7.5 & 12.5 & 17.5 & 22.5 & 27.5 & 32.5 & 37.5 & 45.0 & 62.5 & 87.5 & 125 \\
\hline $\begin{array}{l}0.25 \\
1 \\
12 \\
14 \\
20 \\
23 \\
25 \\
35.25\end{array}$ & $\begin{array}{l}1.8 \\
1.6 \\
1.6 \\
1.2 \\
0.75 \\
1.4 \\
2.5 \\
38\end{array}$ & $\begin{array}{l}0.29 \\
0.15 \\
0.19 \\
0.16 \\
0.12 \\
0.48 \\
1.3 \\
26\end{array}$ & $\begin{array}{l}0.02 \\
0.01 \\
0.03 \\
0.02 \\
0.02 \\
0.20 \\
0.88 \\
13\end{array}$ & $\begin{array}{l}0.01 \\
0 \\
0.02 \\
0.01 \\
0.01 \\
0.12 \\
0.83 \\
7.9\end{array}$ & $\begin{array}{l}0 \\
0 \\
0 \\
0 \\
0 \\
0.03 \\
0.62 \\
4.0\end{array}$ & $\begin{array}{l}0 \\
0 \\
0 \\
0 \\
0 \\
0.01 \\
0.30 \\
1.4\end{array}$ & $\begin{array}{l}0 \\
0 \\
0 \\
0 \\
0 \\
0 \\
.18 \\
.56\end{array}$ & $\begin{array}{l}0 \\
0 \\
0 \\
0 \\
0 \\
0 \\
.05 \\
.19\end{array}$ & $\begin{array}{l}0 \\
0 \\
0 \\
0 \\
0 \\
0 \\
.04 \\
.18\end{array}$ & $\begin{array}{l}0 \\
0 \\
0 \\
0 \\
0 \\
0 \\
0 \\
.02\end{array}$ & $\begin{array}{l}0 \\
0 \\
0 \\
0 \\
0 \\
0 \\
0 \\
0\end{array}$ & $\begin{array}{l}0 \\
0 \\
0 \\
0 \\
0 \\
0 \\
0 \\
0\end{array}$ \\
\hline & & & & & & & & & & & & \\
\hline
\end{tabular}

Note: Alum dose $=8.0 \mathrm{mg} / \mathrm{L}, G=25 \mathrm{~s}^{-1}, t=30 \mathrm{minutes}$ 
Table C-14 - Effluent Number of Particles per ml for Filter No. $2(.50 \mathrm{~mm})$

\begin{tabular}{|c|c|c|c|c|c|c|c|c|c|c|c|c|}
\hline Time & & & Average & Parti & Le size & , micr & cometer & & & & & \\
\hline$(h \times s)$ & 3.5 & 7.5 & 12.5 & 17.5 & 22.5 & 27.5 & 32.5 & 37.5 & 45.0 & 62.5 & 87.5 & 125 \\
\hline $\begin{array}{l}0.25 \\
1 \\
3 \\
5 \\
7 \\
12 \\
14 \\
16 \\
19 \\
22 \\
23 \\
24 \\
34 \\
35 \\
36 \\
37 \\
38\end{array}$ & $\begin{array}{r}6.4 \\
6.6 \\
5.9 \\
4.5 \\
4.7 \\
4.5 \\
2.8 \\
2.6 \\
2.6 \\
1.1 \\
4.0 \\
4.5 \\
9.4 \\
13 \\
22 \\
60 \\
174\end{array}$ & $\begin{array}{l}1.5 \\
1.1 \\
0.66 \\
0.60 \\
0.76 \\
0.46 \\
0.54 \\
0.39 \\
0.36 \\
0.49 \\
0.84 \\
1.4 \\
6.7 \\
13 \\
23 \\
73 \\
203\end{array}$ & $\begin{array}{r}0.27 \\
0.15 \\
0.09 \\
0.10 \\
0.11 \\
0.08 \\
0.27 \\
0.06 \\
0.09 \\
0.34 \\
0.57 \\
0.39 \\
5.8 \\
12 \\
18 \\
60 \\
147\end{array}$ & $\begin{array}{l}0.09 \\
0.07 \\
0.09 \\
0.03 \\
0.07 \\
0.08 \\
0.09 \\
0.05 \\
0.06 \\
0.21 \\
0.50 \\
0.26 \\
4.7 \\
9.6 \\
15 \\
45 \\
103\end{array}$ & $\begin{array}{c}0.03 \\
0.05 \\
0.09 \\
0.02 \\
0.05 \\
0.04 \\
0.04 \\
0 \\
0.04 \\
0.07 \\
0.24 \\
0.17 \\
3.0 \\
6.5 \\
8.5 \\
26 \\
57\end{array}$ & $\begin{array}{c}0 \\
0 \\
0.04 \\
0 \\
0.05 \\
0 \\
0.02 \\
0 \\
0.01 \\
0.02 \\
0.06 \\
0.05 \\
1.2 \\
2.6 \\
3.3 \\
11 \\
24\end{array}$ & $\begin{array}{c}0 \\
0 \\
0 \\
0 \\
0 \\
0 \\
0.01 \\
0 \\
0 \\
0.01 \\
0.04 \\
0 \\
0.51 \\
1.2 \\
1.3 \\
5.6 \\
12\end{array}$ & $\begin{array}{c}0 \\
0 \\
0 \\
0 \\
0 \\
0 \\
0 \\
0 \\
0 \\
0 \\
0.02 \\
0 \\
0.12 \\
0.42 \\
0.37 \\
2.0 \\
4.2\end{array}$ & $\begin{array}{l}0 \\
0 \\
0 \\
0 \\
0 \\
0 \\
0 \\
0 \\
0 \\
0 \\
0 \\
0 \\
0.13 \\
0.28 \\
0.37 \\
2.2 \\
5.5\end{array}$ & $\begin{array}{c}0 \\
0 \\
0 \\
0 \\
0 \\
0 \\
0 \\
0 \\
0 \\
0 \\
0 \\
0 \\
0 \\
0.02 \\
0.02 \\
0.26 \\
0.92\end{array}$ & $\begin{array}{l}0 \\
0 \\
0 \\
0 \\
0 \\
0 \\
0 \\
0 \\
0 \\
0 \\
0 \\
0 \\
0 \\
0 \\
0 \\
0 \\
0\end{array}$ & $\begin{array}{l}0 \\
0 \\
0 \\
0 \\
0 \\
0 \\
0 \\
0 \\
0 \\
0 \\
0 \\
0 \\
0 \\
0 \\
0 \\
0 \\
0\end{array}$ \\
\hline
\end{tabular}

Note: Alum dose $=8.0 \mathrm{mg} / \mathrm{L}, G=100 \mathrm{~s}^{-1}, t=30$ minutes 
Table C-15 _ Effluent Number of Particles per ml for Filter No. 2 (.50 mm)

\begin{tabular}{|c|c|c|c|c|c|c|c|c|c|c|c|c|}
\hline Time & & & verage & Parti & e size & , micr & ometer & & & & & \\
\hline$(h \times s)$ & 3.5 & 7.5 & 12.5 & 17.5 & 22.5 & 27.5 & 32.5 & 37.5 & 45.0 & 62.5 & 87.5 & 125 \\
\hline $\begin{array}{l}0.25 \\
1 \\
2 \\
3 \\
4 \\
7 \\
9 \\
11 \\
14\end{array}$ & $\begin{array}{r}13 \\
7.8 \\
6.2 \\
3.7 \\
3.0 \\
1.7 \\
1.5 \\
5.5 \\
9.3\end{array}$ & $\begin{array}{l}2.7 \\
2.1 \\
2.1 \\
0.99 \\
0.68 \\
0.35 \\
0.67 \\
2.0 \\
1.9\end{array}$ & $\begin{array}{l}0.23 \\
0.76 \\
0.25 \\
0.27 \\
0.09 \\
0.06 \\
0.28 \\
0.60 \\
1.8\end{array}$ & $\begin{array}{l}0.06 \\
0.28 \\
0.11 \\
0.08 \\
0.03 \\
0.03 \\
0.09 \\
0.33 \\
1.9\end{array}$ & $\begin{array}{l}0 \\
0.14 \\
0.03 \\
0.07 \\
0 \\
0 \\
0.04 \\
0.17 \\
1.0\end{array}$ & $\begin{array}{l}0 \\
0 \\
.01 \\
.01 \\
0 \\
0 \\
.03 \\
.11 \\
.57\end{array}$ & $\begin{array}{l}0 \\
0 \\
0 \\
0 \\
0 \\
0 \\
.01 \\
.03 \\
.47\end{array}$ & $\begin{array}{l}0 \\
0 \\
0 \\
0 \\
0 \\
0 \\
.01 \\
.02 \\
.09\end{array}$ & $\begin{array}{l}0 \\
0 \\
0 \\
0 \\
0 \\
0 \\
0 \\
.01 \\
.09\end{array}$ & $\begin{array}{l}0 \\
0 \\
0 \\
0 \\
0 \\
0 \\
0 \\
0 \\
0\end{array}$ & $\begin{array}{l}0 \\
0 \\
0 \\
0 \\
0 \\
0 \\
0 \\
0 \\
0\end{array}$ & $\begin{array}{l}0 \\
0 \\
0 \\
0 \\
0 \\
0 \\
0 \\
0 \\
0\end{array}$ \\
\hline
\end{tabular}

Note: Alum dose $=8.0 \mathrm{mg} / \mathrm{L}, \mathrm{G}=50 \mathrm{~s}^{-1}, \mathrm{t}=15 \mathrm{minutes}$ 
Table C-16 - Effluent Number of Particles per ml for Filter No. 2 (.50 mm)

\begin{tabular}{|c|c|c|c|c|c|c|c|c|c|c|c|c|}
\hline Time & & & Average & Partic & le Size & $\Rightarrow$, micr & omete & & & & & \\
\hline (hrs) & 3.5 & 7.5 & 12.5 & 17.5 & 22.5 & 27.5 & 32.5 & 37.5 & 45.0 & 62.5 & 87.5 & 125 \\
\hline $\begin{array}{l}0.25 \\
1 \\
2 \\
3 \\
4 \\
6 \\
13 \\
24 \\
26 \\
28 \\
30 \\
32 \\
36 \\
45\end{array}$ & $\begin{array}{c}2.0 \\
2.1 \\
1.3 \\
1.2 \\
1.0 \\
1.0 \\
2.1 \\
30 \\
59 \\
44 \\
62 \\
83 \\
82 \\
694\end{array}$ & $\begin{array}{c}1.1 \\
0.92 \\
0.41 \\
0.52 \\
0.43 \\
0.36 \\
0.46 \\
21 \\
39 \\
30 \\
48 \\
64 \\
74 \\
654\end{array}$ & $\begin{array}{c}0.19 \\
0.24 \\
0.10 \\
0.21 \\
0.11 \\
0.20 \\
0.02 \\
18 \\
26 \\
17 \\
30 \\
43 \\
53 \\
388\end{array}$ & $\begin{array}{r}0.04 \\
0.12 \\
0.03 \\
0.07 \\
0.09 \\
0.10 \\
0.03 \\
16 \\
21 \\
13 \\
24 \\
36 \\
43 \\
249\end{array}$ & $\begin{array}{c}0 \\
0 \\
0 \\
0 \\
0.04 \\
0.02 \\
0 \\
11 \\
15 \\
11 \\
17 \\
26 \\
30 \\
138\end{array}$ & $\begin{array}{c}0 \\
0 \\
0 \\
0 \\
0 \\
0.01 \\
0 \\
5.6 \\
6.9 \\
5.5 \\
8.2 \\
12 \\
16 \\
59\end{array}$ & $\begin{array}{c}0 \\
0 \\
0 \\
0 \\
0 \\
0 \\
0 \\
2.6 \\
2.9 \\
2.8 \\
4.4 \\
7.1 \\
9.3 \\
29\end{array}$ & $\begin{array}{l}0 \\
0 \\
0 \\
0 \\
0 \\
0 \\
0 \\
0.79 \\
1.0 \\
1.1 \\
1.4 \\
3.0 \\
3.9 \\
10\end{array}$ & $\begin{array}{l}0 \\
0 \\
0 \\
0 \\
0 \\
0 \\
0 \\
0.94 \\
1.0 \\
1.2 \\
2.1 \\
4.1 \\
5.1 \\
9.7\end{array}$ & $\begin{array}{l}0 \\
0 \\
0 \\
0 \\
0 \\
0 \\
0 \\
0.14 \\
0.14 \\
0.13 \\
0.30 \\
0.64 \\
1.2 \\
1.2\end{array}$ & $\begin{array}{ll} & 0 \\
& 0 \\
& 0 \\
& 0 \\
& 0 \\
& 0 \\
& 0 \\
& 0 \\
4 & 0 \\
4 & 0 \\
3 & 0 \\
0 & 0 \\
4 & 0 \\
& 0 \\
& 01\end{array}$ & $\begin{array}{l}0 \\
0 \\
0 \\
0 \\
0 \\
0 \\
0 \\
0 \\
0 \\
0 \\
0 \\
0 \\
0 \\
0\end{array}$ \\
\hline
\end{tabular}

Note: Alum dose $=8.0 \mathrm{mg} / \mathrm{L}, G=50 \mathrm{~s}^{-1}, t=45 \mathrm{minutes}$ 
Table C-17 - Effluent Number of Particles per ml for Filter No. 3 (1.00 mm)

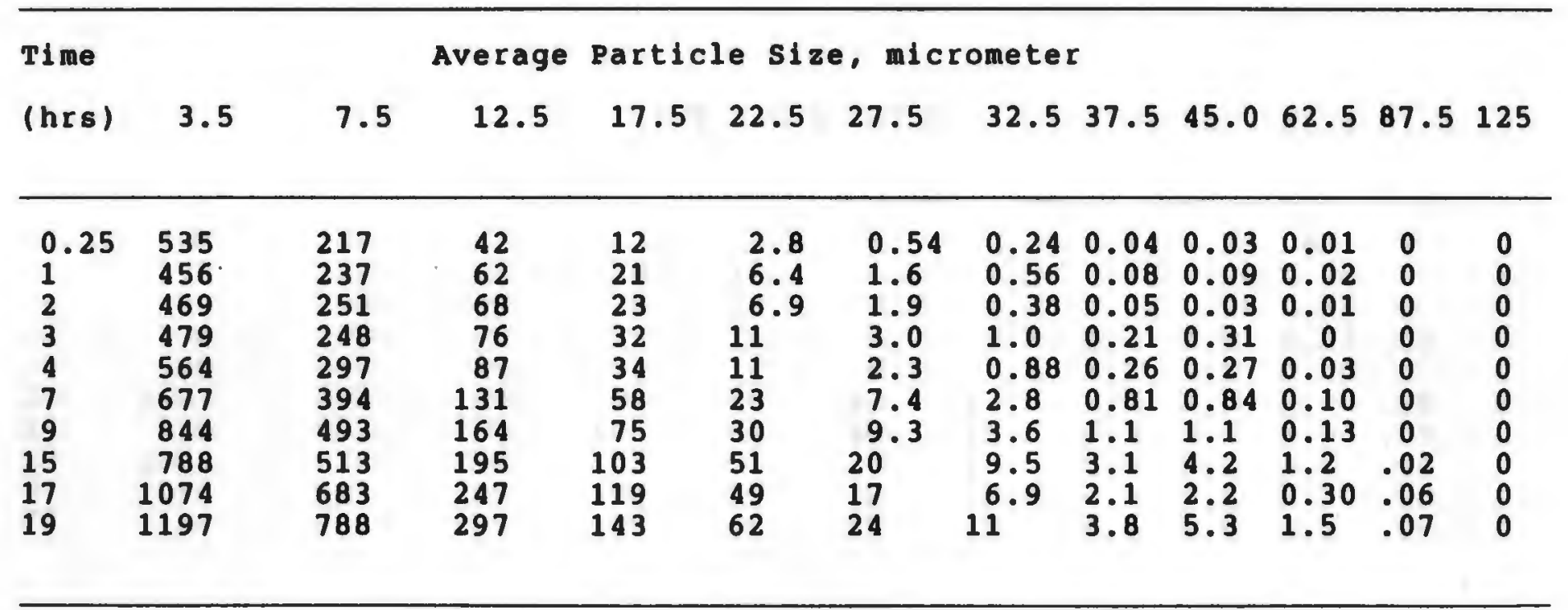

Note: Alum dose=0.0 mg/L, $G=50 \mathrm{~s}^{-1}, t=30 \mathrm{minutes}$ 
Table C-18 - Effluent Number of Particles per ml for Filter No. 3 (1.00 mm)

\begin{tabular}{|c|c|c|c|c|c|c|c|c|c|c|c|c|}
\hline Time & & & Average & Partic & le size & $\Rightarrow, m i c$ & romete & & & & & \\
\hline (hrs) & 3.5 & 7.5 & 12.5 & 17.5 & 22.5 & 27.5 & 32.5 & 37.5 & 45.0 & 62.5 & 87.5 & 125 \\
\hline $\begin{array}{l}0.25 \\
1 \\
4 \\
6 \\
9 \\
20 \\
23 \\
26 \\
27 \\
28\end{array}$ & $\begin{array}{r}405 \\
338 \\
257 \\
236 \\
279 \\
1001 \\
938 \\
1004 \\
1096 \\
1124\end{array}$ & $\begin{array}{l}174 \\
133 \\
130 \\
131 \\
137 \\
493 \\
561 \\
578 \\
613 \\
627\end{array}$ & $\begin{array}{r}42 \\
60 \\
40 \\
48 \\
44 \\
149 \\
202 \\
198 \\
202 \\
205\end{array}$ & $\begin{array}{r}12 \\
28 \\
18 \\
27 \\
23 \\
73 \\
105 \\
102 \\
108 \\
115\end{array}$ & $\begin{array}{l}3.1 \\
15 \\
10 \\
16 \\
14 \\
46 \\
62 \\
61 \\
59 \\
68\end{array}$ & $\begin{array}{l}0.54 \\
4.4 \\
3.7 \\
6.9 \\
6.7 \\
23 \\
30 \\
29 \\
30 \\
33\end{array}$ & $\begin{array}{l}0.19 \\
1.6 \\
1.4 \\
3.6 \\
3.2 \\
14 \\
16 \\
17 \\
16 \\
18\end{array}$ & $\begin{array}{l}0.16 \\
0.72 \\
0.43 \\
1.3 \\
1.1 \\
6.0 \\
6.3 \\
6.2 \\
6.4 \\
6.8\end{array}$ & $\begin{array}{l}0.05 \\
0.28 \\
0.55 \\
1.9 \\
1.8 \\
9.0 \\
9.9 \\
8.9 \\
9.5 \\
9.8\end{array}$ & $\begin{array}{l}0 \\
0.08 \\
0.03 \\
0.33 \\
0.40 \\
3.4 \\
2.9 \\
3.1 \\
2.2 \\
3.2\end{array}$ & $\begin{array}{l}0 \\
0 \\
0 \\
.03 \\
0 \\
.28 \\
.27 \\
.30 \\
.20 \\
.29\end{array}$ & $\begin{array}{l}0 \\
0 \\
0 \\
0 \\
0 \\
0 \\
0 \\
0 \\
0 \\
0\end{array}$ \\
\hline
\end{tabular}

Note: Alum dose $=2.0 \mathrm{mg} / \mathrm{L}, G=50 \mathrm{~s}^{-1}, t=30 \mathrm{minutes}$ 
Table C-19 - Effluent Number of Particles per ml for Filter No. 3 (1.00 mm)

\begin{tabular}{|c|c|c|c|c|c|c|c|c|c|c|c|c|}
\hline Time & & & Average & Partic & le size & e, micr & rometer & & & & & \\
\hline (hrs) & 3.5 & 7.5 & 12.5 & 17.5 & 22.5 & 27.5 & 32.5 & 37.5 & 45.0 & 62.5 & 87.5 & 125 \\
\hline $\begin{array}{l}0.25 \\
1 \\
3 \\
6 \\
8 \\
18 \\
19\end{array}$ & $\begin{array}{l}118 \\
106 \\
107 \\
129 \\
316 \\
715 \\
995\end{array}$ & $\begin{array}{r}57 \\
53 \\
48 \\
63 \\
178 \\
350 \\
528\end{array}$ & $\begin{array}{r}14 \\
15 \\
14 \\
15 \\
62 \\
95 \\
142\end{array}$ & $\begin{array}{l}4.4 \\
6.3 \\
6.6 \\
5.7 \\
27 \\
44 \\
62\end{array}$ & $\begin{array}{l}1.1 \\
2.8 \\
3.9 \\
2.7 \\
15 \\
26 \\
34\end{array}$ & $\begin{array}{l}0.18 \\
1.0 \\
1.4 \\
1.3 \\
7.8 \\
14 \\
16\end{array}$ & $\begin{array}{l}0.04 \\
0.48 \\
1.1 \\
0.60 \\
5.1 \\
8.6 \\
10\end{array}$ & $\begin{array}{l}0 \\
0.20 \\
0.42 \\
0.31 \\
1.9 \\
3.5 \\
4.3\end{array}$ & $\begin{array}{l}0 \\
0.23 \\
0.51 \\
0.49 \\
3.9 \\
5.9 \\
5.9\end{array}$ & $\begin{array}{l}0 \\
0.01 \\
0.11 \\
0.14 \\
1.4 \\
2.3 \\
1.8\end{array}$ & $\begin{array}{l}0 \\
0 \\
0 \\
0 \\
.07 \\
18 \\
.12\end{array}$ & $\begin{array}{l}0 \\
0 \\
0 \\
0 \\
0 \\
0 \\
0\end{array}$ \\
\hline
\end{tabular}

Note: Alum dose $=8.0 \mathrm{mg} / \mathrm{L}, G=50 \mathrm{~s}^{-1}, t=30 \mathrm{minutes}$ 
Table c-20. Effluent Number of Particles per ml for Filter No. 3 (1.00 mm)

\begin{tabular}{|c|c|c|c|c|c|c|c|c|c|c|c|c|}
\hline \multirow{2}{*}{$\begin{array}{l}\text { Time } \\
\text { (hrs) }\end{array}$} & \multirow[b]{2}{*}{3.5} & \multirow[b]{2}{*}{7.5} & \multirow{2}{*}{$\begin{array}{c}\text { Average } \\
12.5\end{array}$} & \multicolumn{4}{|c|}{ Particle size, micrometer } & \multirow{2}{*}{37.5} & \multirow[b]{2}{*}{45.0} & \multirow[b]{2}{*}{62.5} & \multirow[b]{2}{*}{87.5} & \multirow[b]{2}{*}{125} \\
\hline & & & & 17.5 & 22.5 & 27.5 & 32.5 & & & & & \\
\hline $\begin{array}{c}0.25 \\
1 \\
3 \\
6 \\
9 \\
12 \\
13\end{array}$ & $\begin{array}{r}32 \\
93 \\
79 \\
184 \\
407 \\
927 \\
1380\end{array}$ & $\begin{array}{r}14 \\
42 \\
42 \\
98 \\
208 \\
473 \\
793\end{array}$ & $\begin{array}{c}3.2 \\
9.8 \\
12 \\
27 \\
52 \\
118 \\
216\end{array}$ & $\begin{array}{l}1.2 \\
3.5 \\
4.6 \\
11 \\
20 \\
46 \\
84\end{array}$ & $\begin{array}{l}0.37 \\
1.4 \\
2.1 \\
4.8 \\
9.7 \\
22 \\
42\end{array}$ & $\begin{array}{l}0.16 \\
0.59 \\
0.87 \\
2.0 \\
4.6 \\
10 \\
21\end{array}$ & $\begin{array}{l}0.08 \\
0.34 \\
0.48 \\
1.1 \\
3.1 \\
7.1 \\
12\end{array}$ & $\begin{array}{l}0.01 \\
0.08 \\
0.25 \\
0.58 \\
1.5 \\
3.3 \\
5.0\end{array}$ & $\begin{array}{l}0 \\
0.13 \\
0.29 \\
0.67 \\
2.1 \\
4.8 \\
7.8\end{array}$ & $\begin{array}{l}0 \\
0.04 \\
0.15 \\
0.35 \\
0.91 \\
2.1 \\
2.8\end{array}$ & $\begin{array}{l}0 \\
0 \\
.01 \\
.02 \\
.06 \\
.14 \\
.21\end{array}$ & $\begin{array}{l}0 \\
0 \\
0 \\
0 \\
0 \\
0 \\
0\end{array}$ \\
\hline
\end{tabular}

Note: Alum dose $=16.0 \mathrm{mg} / \mathrm{L}, G=50 \mathrm{~s}^{-1}, t=30 \mathrm{minutes}$ 
Table C-21 - Effluent Number of Particles per ml for Filter No. 3 (1.00 mm)

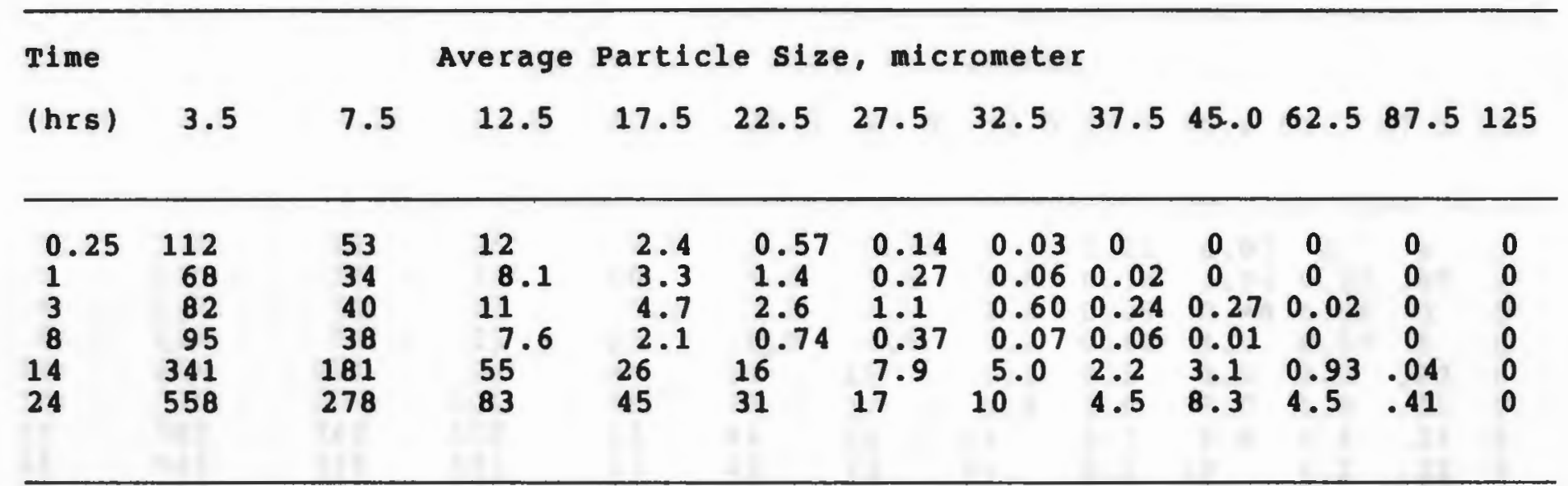

Note: Alum dose $=8.0 \mathrm{mg} / \mathrm{L}, G=25 \mathrm{~s}^{-1}, t=30 \mathrm{minutes}$ 
Table C-22 - Effluent Number of Particles per ml for Filter No. 3 ( $1.00 \mathrm{~mm})$

\begin{tabular}{|c|c|c|c|c|c|c|c|c|c|c|c|c|}
\hline Time & & & Average & Partic & le size & e, $\mathrm{mics}$ & comete & & & & & \\
\hline$(h r s)$ & 3.5 & 7.5 & 12.5 & 17.5 & 22.5 & 27.5 & 32.5 & 37.5 & 45.0 & 62.58 & 87.5 & 125 \\
\hline $\begin{array}{l}0.25 \\
1 \\
4 \\
6 \\
16 \\
18 \\
19 \\
21 \\
23 \\
25 \\
28\end{array}$ & $\begin{array}{r}146 \\
121 \\
127 \\
114 \\
636 \\
729 \\
782 \\
944 \\
1153 \\
1117 \\
1240\end{array}$ & $\begin{array}{r}81 \\
56 \\
51 \\
59 \\
291 \\
355 \\
369 \\
419 \\
511 \\
533 \\
642\end{array}$ & $\begin{array}{l}25 \\
19 \\
13 \\
22 \\
81 \\
101 \\
109 \\
121 \\
143 \\
159 \\
211\end{array}$ & $\begin{array}{c}9.9 \\
10 \\
5.4 \\
13 \\
44 \\
55 \\
63 \\
63 \\
71 \\
87 \\
122\end{array}$ & $\begin{array}{l}3.2 \\
4.8 \\
3.1 \\
6.9 \\
29 \\
33 \\
41 \\
43 \\
44 \\
54 \\
74\end{array}$ & $\begin{array}{l}0.73 \\
1.6 \\
1.9 \\
4.6 \\
15 \\
17 \\
22 \\
23 \\
23 \\
28 \\
38\end{array}$ & $\begin{array}{l}0.39 \\
1.0 \\
1.1 \\
2.1 \\
9.4 \\
9.4 \\
14 \\
16 \\
16 \\
16 \\
22\end{array}$ & $\begin{array}{l}0.11 \\
0.34 \\
0.29 \\
0.91 \\
4.3 \\
3.8 \\
5.7 \\
6.2 \\
6.8 \\
6.4 \\
9.5\end{array}$ & $\begin{array}{l}0.07 \\
0.54 \\
0.48 \\
1.4 \\
6.2 \\
5.7 \\
8.8 \\
10 \\
10 \\
9.9 \\
12\end{array}$ & $\begin{array}{l}0 \\
0.31 \\
0.08 \\
0.27 \\
2.2 \\
1.9 \\
3.3 \\
4.2 \\
4.5 \\
4.0 \\
4.4\end{array}$ & $\begin{array}{l}0 \\
.05 \\
0 \\
0 \\
.22 \\
.07 \\
.34 \\
.25 \\
.23 \\
.43 \\
.40\end{array}$ & $\begin{array}{l}0 \\
0 \\
0 \\
0 \\
0 \\
0 \\
0 \\
0 \\
0 \\
0 \\
0\end{array}$ \\
\hline
\end{tabular}

Note: Alum dose=8.0 mg/L, $G=100 \mathrm{~s}^{-1}, \mathrm{t}=30$ minutes 
Table C-23 - Effluent Number of Particles per ml for Filter No. 3 (1.00 $\mathrm{mm})$

\begin{tabular}{|c|c|c|c|c|c|c|c|c|c|c|c|c|}
\hline Time & & & Average & Particl & Le size & $e, m i c$ & cometer & & & & & \\
\hline (hrs) & 3.5 & 7.5 & 12.5 & 17.5 & 22.5 & 27.5 & 32.5 & 37.5 & 45.0 & 62.5 & 87.5 & 125 \\
\hline $\begin{array}{l}0.25 \\
1 \\
2 \\
3 \\
4 \\
7 \\
9 \\
14\end{array}$ & $\begin{array}{r}201 \\
145 \\
160 \\
165 \\
440 \\
631 \\
858 \\
1209\end{array}$ & $\begin{array}{r}86 \\
76 \\
53 \\
38 \\
115 \\
235 \\
393 \\
758\end{array}$ & $\begin{array}{c}20 \\
18 \\
9.8 \\
8.6 \\
18 \\
35 \\
98 \\
262\end{array}$ & $\begin{array}{c}7.4 \\
6.5 \\
5.1 \\
0 \\
6.9 \\
7.4 \\
33 \\
123\end{array}$ & $\begin{array}{c}3.1 \\
2.0 \\
3.3 \\
0 \\
1.3 \\
2.5 \\
15 \\
65\end{array}$ & $\begin{array}{c}0.87 \\
1.5 \\
0.36 \\
0 \\
1.3 \\
0.53 \\
5.6 \\
31\end{array}$ & $\begin{array}{c}0.16 \\
0.50 \\
0 \\
0 \\
0.66 \\
0 \\
2.6 \\
21^{2.6}\end{array}$ & $\begin{array}{c}0 \\
0 \\
0 \\
0 \\
0 \\
0 \\
1.3 \\
7.3\end{array}$ & $\begin{array}{c}0 \\
0 \\
0 \\
0 \\
0 \\
0 \\
13^{1} \cdot 6\end{array}$ & $\begin{array}{l}0 \\
0 \\
0 \\
0 \\
0 \\
0 \\
0.68 \\
5.1\end{array}$ & $\begin{array}{l}0 \\
0 \\
0 \\
0 \\
0 \\
0 \\
0 \\
0 \\
0 \\
.34\end{array}$ & $\begin{array}{l}0 \\
0 \\
0 \\
0 \\
0 \\
0 \\
0 \\
0\end{array}$ \\
\hline
\end{tabular}

Note: Alum dose $=8.0 \mathrm{mg} / \mathrm{L}, G=50 \mathrm{~s}^{-1}, t=15$ minutes 
Table C-24 - Effluent Number of Particles per ml for Filter No. 3 (1.00 mm)

\begin{tabular}{|c|c|c|c|c|c|c|c|c|c|c|c|c|}
\hline Time & & & Average & Partic & le size & e, micr & romete & & & & & \\
\hline (hrs) & 3.5 & 7.5 & 12.5 & 17.5 & 22.5 & 27.5 & 32.5 & 37.5 & 45.0 & 62.5 & 87.5 & 125 \\
\hline $\begin{array}{c}0.25 \\
1 \\
5 \\
8 \\
10 \\
15 \\
17 \\
25 \\
27\end{array}$ & $\begin{array}{r}84 \\
85 \\
128 \\
327 \\
361 \\
634 \\
557 \\
727 \\
1051\end{array}$ & $\begin{array}{r}51 \\
47 \\
38 \\
162 \\
172 \\
337 \\
293 \\
379 \\
578\end{array}$ & $\begin{array}{r}20 \\
16 \\
4.3 \\
44 \\
49 \\
106 \\
92 \\
130 \\
19.8\end{array}$ & $\begin{array}{c}10 \\
7.2 \\
1.3 \\
23 \\
25 \\
54 \\
49 \\
74 \\
114\end{array}$ & $\begin{array}{l}4.2 \\
3.5 \\
0.61 \\
14 \\
16 \\
35 \\
32 \\
43 \\
66\end{array}$ & $\begin{array}{l}1.2 \\
1.8 \\
0.38 \\
5.9 \\
7.6 \\
17 \\
16 \\
21 \\
32\end{array}$ & $\begin{array}{c}0.41 \\
0.52 \\
0.20 \\
3.8 \\
4.3 \\
10 \\
8.4 \\
11 \\
18\end{array}$ & $\begin{array}{l}0.06 \\
0.21 \\
0.07 \\
1.5 \\
1.7 \\
4.0 \\
3.7 \\
4.8 \\
6.5\end{array}$ & $\begin{array}{l}0.06 \\
0.23 \\
0.10 \\
2.0 \\
2.7 \\
5.5 \\
4.8 \\
6.5 \\
9.3\end{array}$ & $\begin{array}{ll} & 0 \\
0.04 & 0.01 \\
0.61 & 0.61 \\
0.59 \\
1.6 \\
1.4 \\
1.7 \\
3.0\end{array}$ & $\begin{array}{l}0 \\
0 \\
0 \\
.02 \\
.04 \\
.09 \\
.11 \\
.18 \\
.14\end{array}$ & $\begin{array}{l}0 \\
0 \\
0 \\
0 \\
0 \\
0 \\
0 \\
0 \\
0\end{array}$ \\
\hline
\end{tabular}

Note: Alum dose $=8.0 \mathrm{mg} / \mathrm{L}, \mathrm{G}=50 \mathrm{~s}^{-1}, t=45 \mathrm{minutes}$ 


\section{APPENDIX D}

Head Loss Graphs 


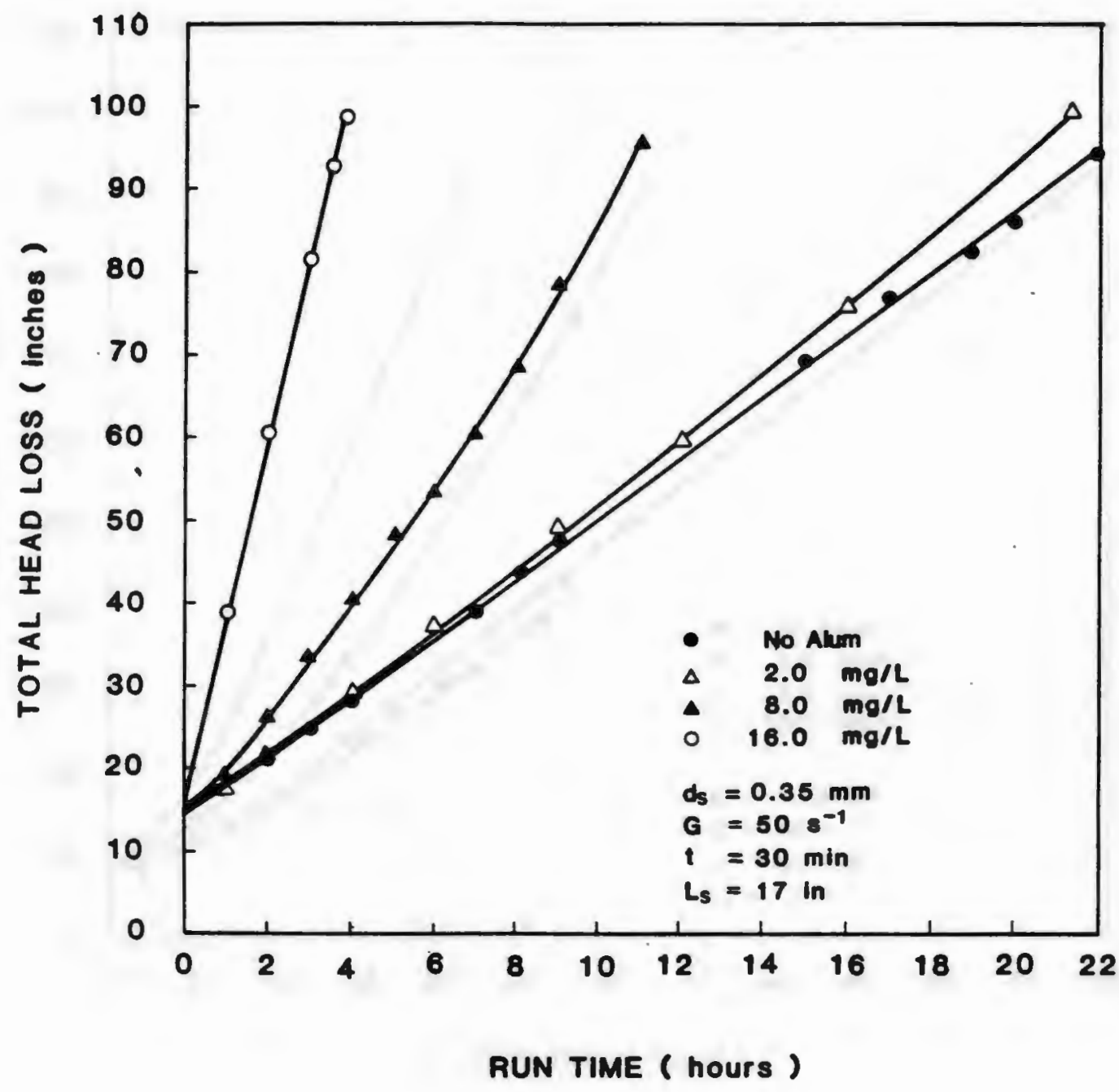

Figure DI Total head loss versus run time for fine sand with various alum dosages. 


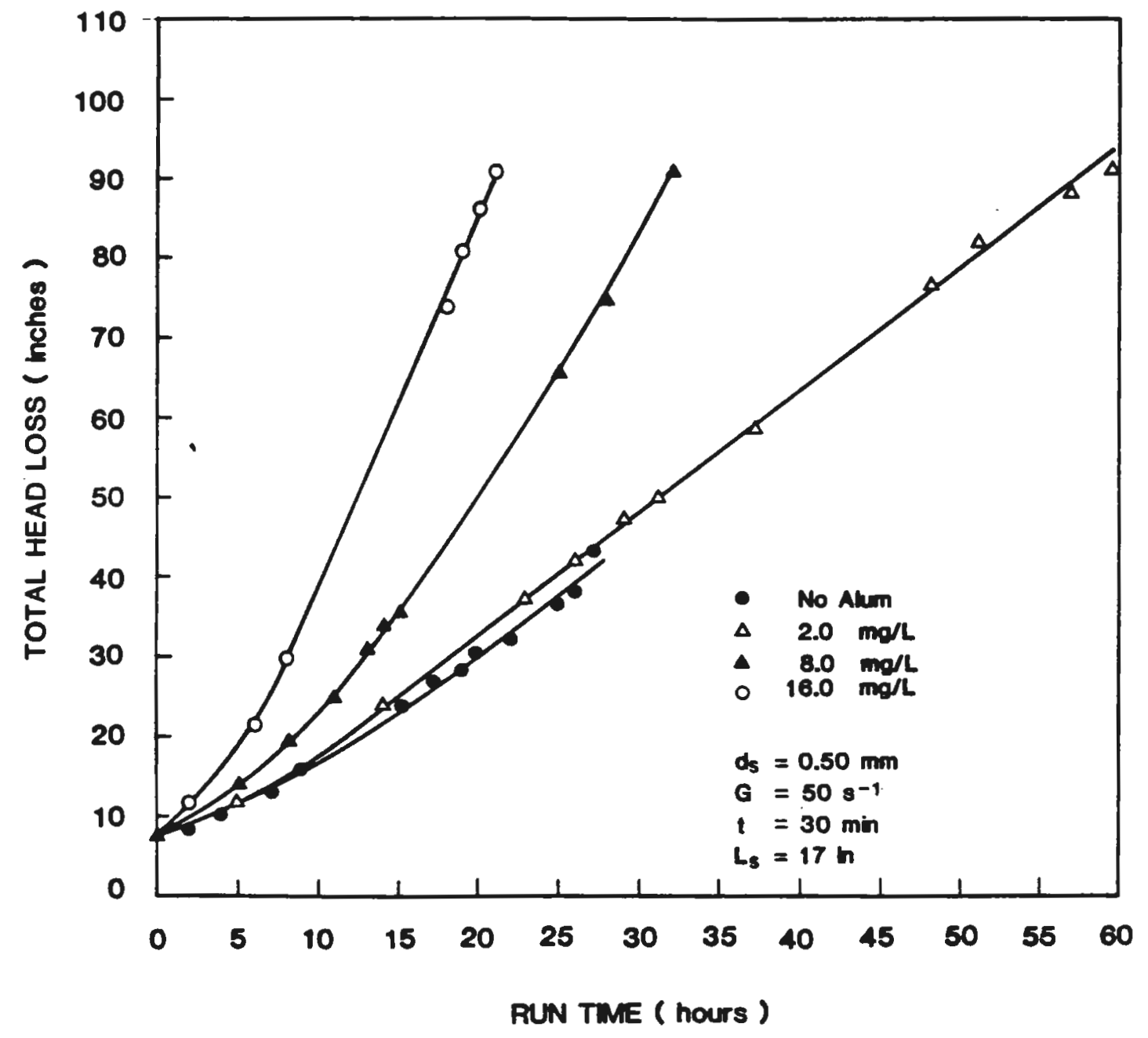

Figure D2 Total head loss versus run time for medium sand with various alum dosages. 


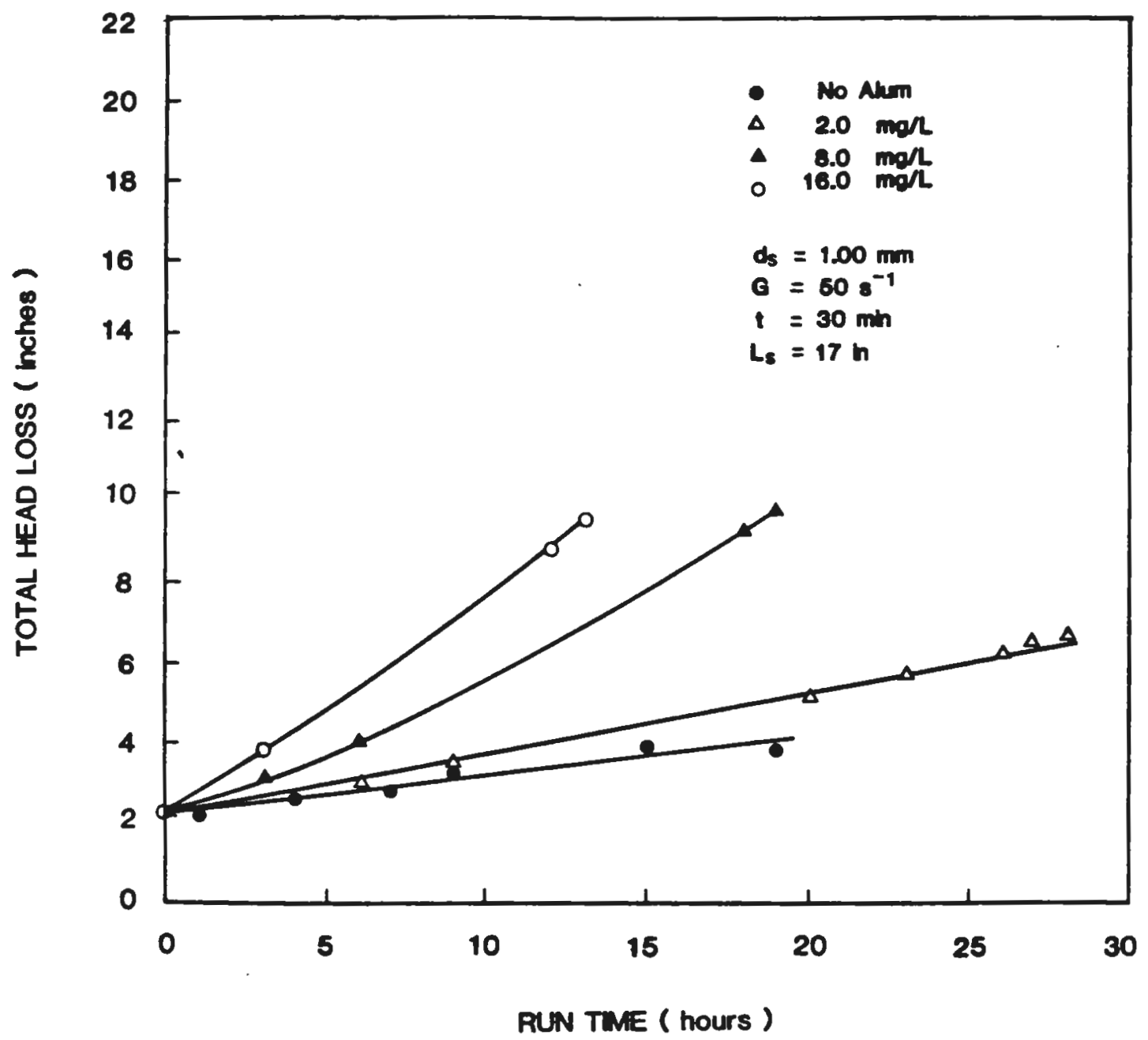

Figure D3 Total head loss versus run time for coarse sand with various alum dosages. 


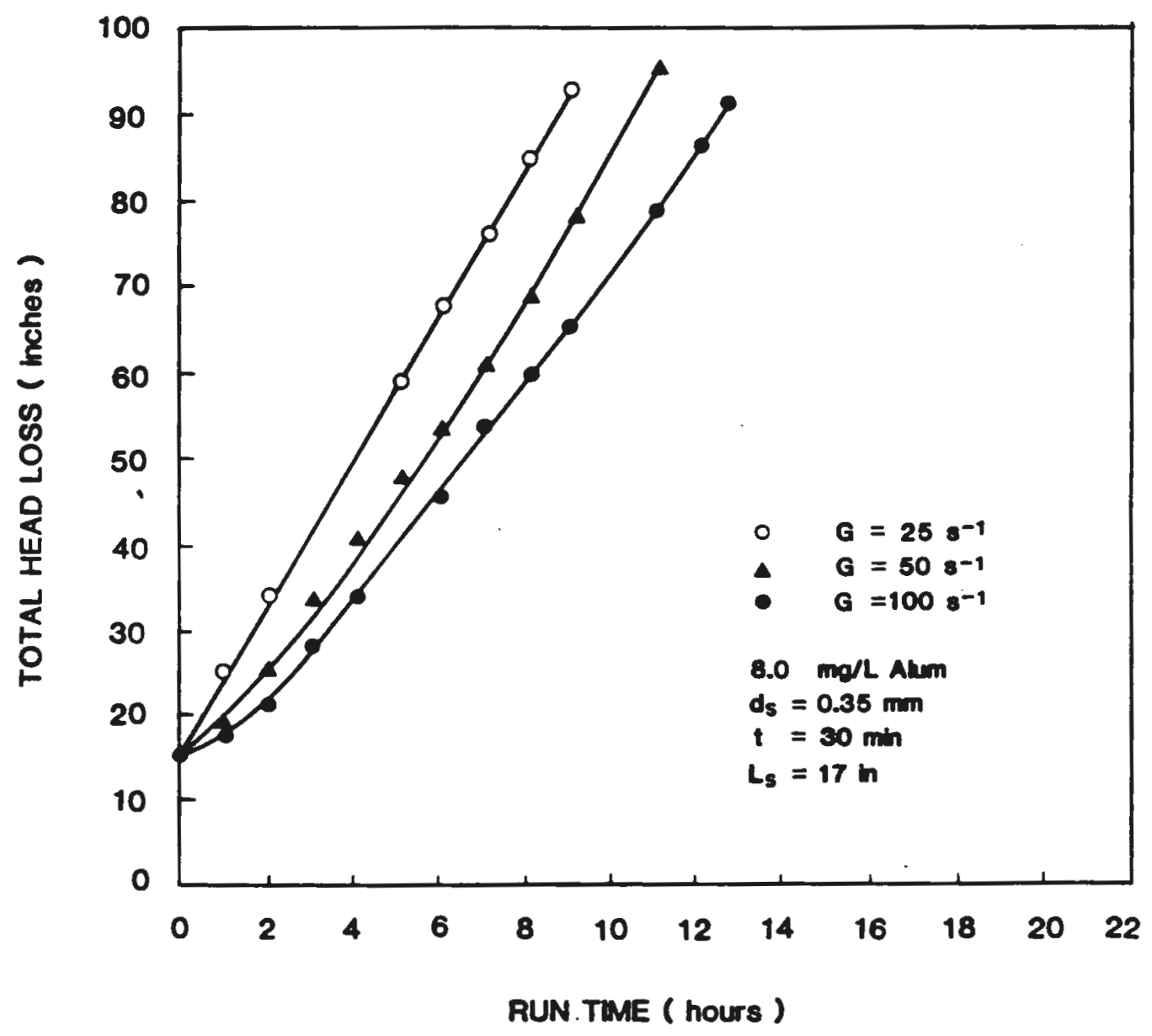

Figure D4 Total head loss versus run time for fine sand with various mixing intensities. 


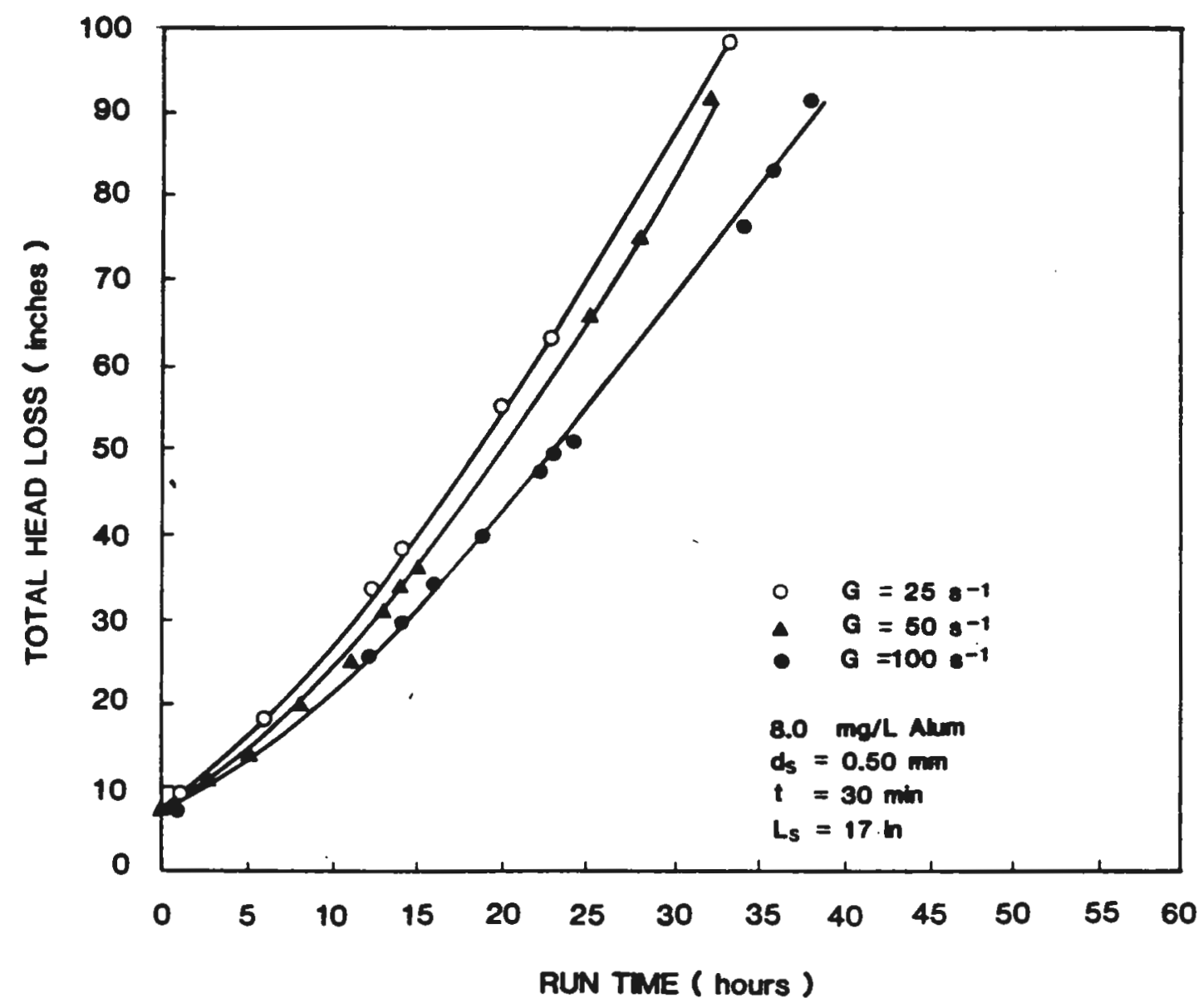

Figure D5 Total head loss versus run time for medium sand with various mixing intensities. 


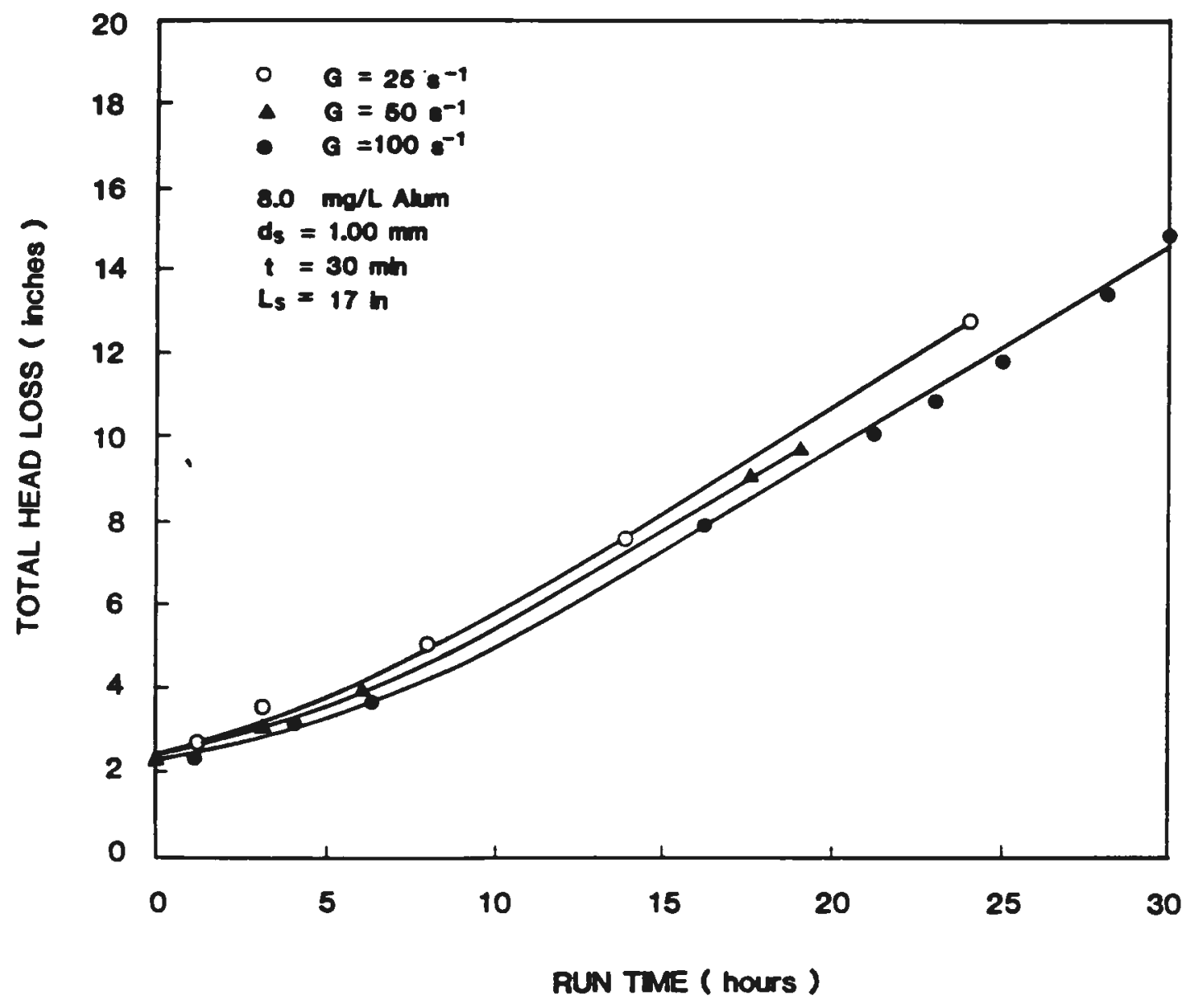

Figure D6 Total head loss versus run time for coarse sand with various mixing intensities. 


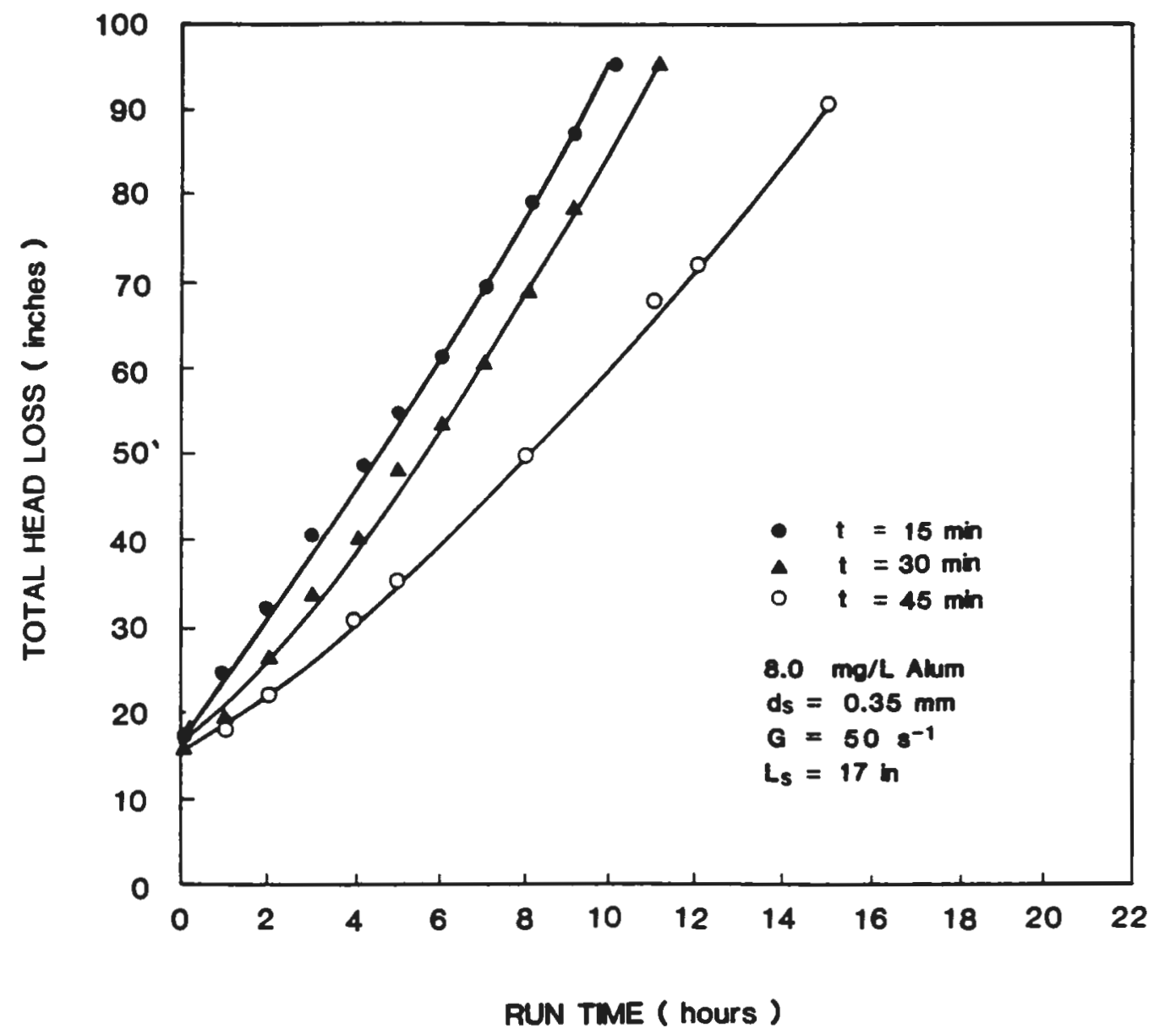

Figure D7 Total head loss versus run time for fine sand with various mixing times. 


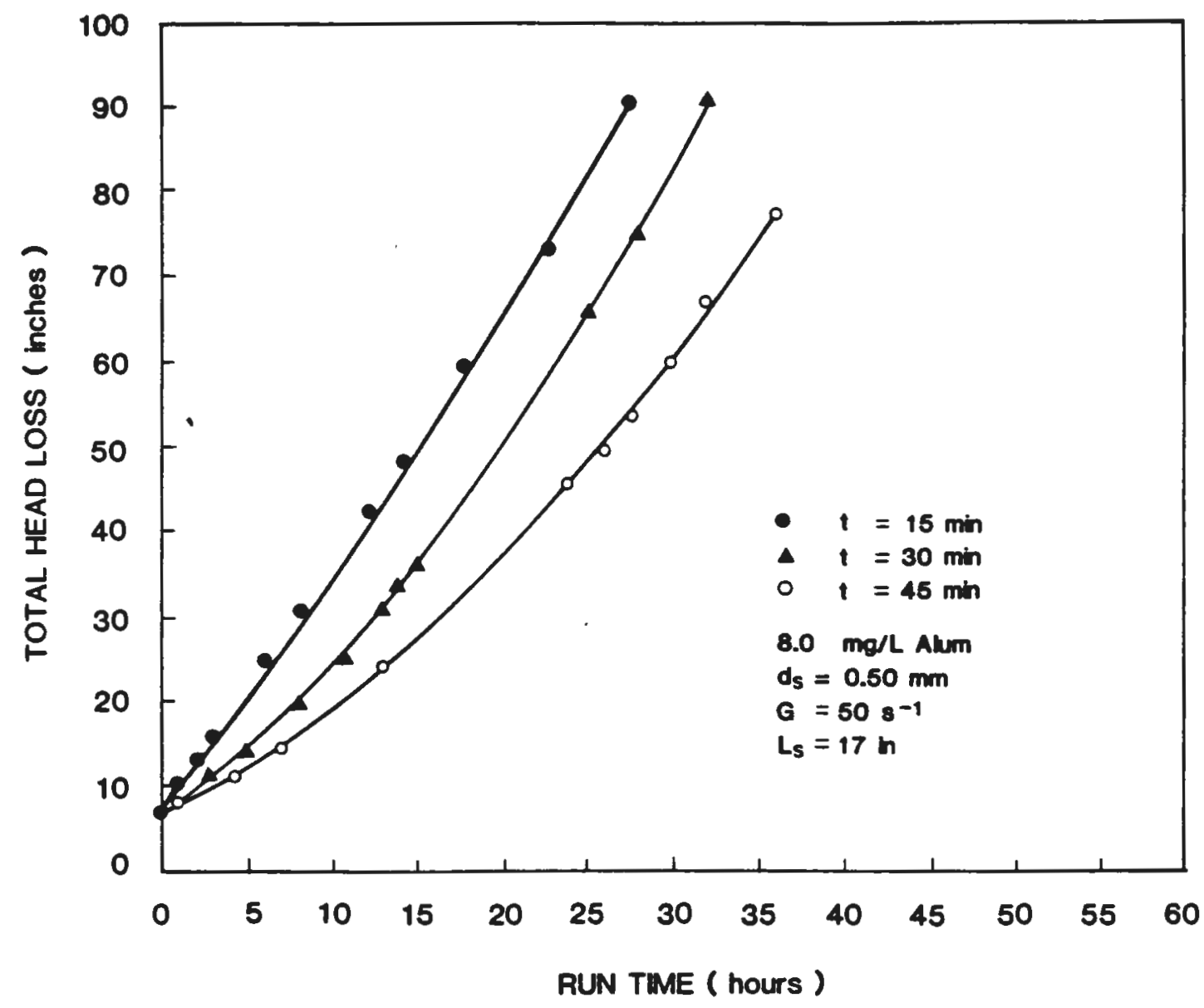

Figure D8 Total head loss versus run time for medium sand with various mixing times. 


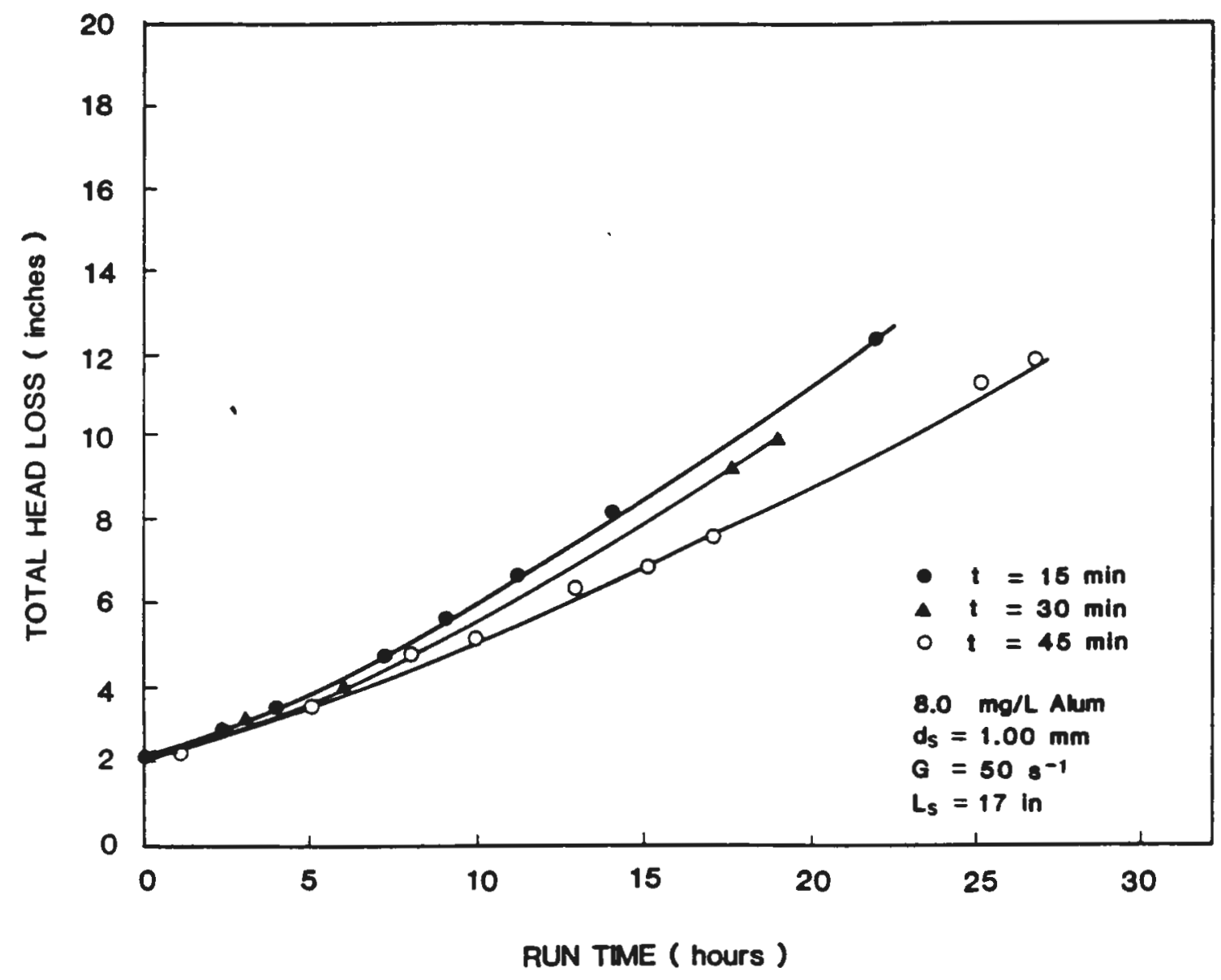

Figure D9 Total head loss versus run time for coarse sand with various mixing times. 


\section{APPENDIX E}

Tables of the Head Loss Rates 
Table E-1 - Rate of Head Loss(in/hr) with Depth and Different Influent Particle size Distributions for Filter No. $1(0.35 \mathrm{~mm})$

\begin{tabular}{|c|c|c|c|c|c|c|c|c|}
\hline $\begin{array}{l}\text { Sand } \\
\text { Depth } \\
(1 \mathrm{n})\end{array}$ & $0 \underset{\text { Alum }}{(1)}$ & $2 \underset{\mathrm{mg} / \mathrm{L}}{(1)}$ & $\begin{array}{c}(1) \\
\mathrm{mg} / \mathrm{Lm}\end{array}$ & $\begin{array}{l}(1) \\
16 \mathrm{mg} / \mathrm{L} \\
\text { Alum }\end{array}$ & $\begin{array}{r}(2) \\
G=25\end{array} \mathrm{~s}^{-1}$ & $\begin{array}{c}(2) \\
G=100\end{array}$ & $s^{-1} t=15$ min & $\begin{array}{l}(3) \\
t=45 \mathrm{~min}\end{array}$ \\
\hline 1 & 3.27 & 3.45 & 6.13 & 20.25 & 7.90 & 2.54 & 6.34 & 4.34 \\
\hline 5 & 3.52 & 3.82 & 7.15 & 21.28 & 8.48 & 6.08 & 7.13 & 4.93 \\
\hline 9 & 3.58 & 3.88 & 7.23 & 21.46 & 8. 51 & 6.13 & 7.27 & 4.99 \\
\hline 13 & 3.59 & 3.88 & 7.26 & 21.56 & 8.51 & 6.15 & 7.36 & 5.00 \\
\hline 17 & 3.60 & 3.88 & 7.29 & 21.87 & 8.52 & 6.16 & 7.50 & 5.01 \\
\hline
\end{tabular}

Note: (1) $G=50 \mathrm{~s}^{-1}, t=30$ minutes

(2) Alum dose $=8.0 \mathrm{mg} / \mathrm{L}, t=30$ minutes

(3) Alum dose=8.0 $\mathrm{mg} / \mathrm{L}, G=50 \mathrm{~s}^{-1}$ 
Table E-2 - Rate of Head Loss(in/hr) With Different Depth and Influent Particle size Distributions for Filtér No. $2(0.50 \mathrm{~mm})$

\begin{tabular}{|c|c|c|c|c|c|c|c|c|}
\hline $\begin{array}{l}\text { Sand } \\
\text { Depth } \\
(\text { in) }\end{array}$ & $\begin{array}{c}\text { (1) } \\
0 \mathrm{mg} / \mathrm{L} \\
\text { Alum }\end{array}$ & $\begin{array}{c}(1) \\
2 \mathrm{mg} / \mathrm{L} \\
\text { Alum }\end{array}$ & $\begin{array}{c}(1) \\
8 \mathrm{mg} / \mathrm{L} \\
\text { Alum }\end{array}$ & $\begin{array}{l}(1) \\
16 \mathrm{mg} / \mathrm{L} \\
\text { Alum }\end{array}$ & $\underset{G=25}{(2)} \mathrm{s}^{-1}$ & $\begin{array}{c}(2) \\
G=100\end{array}$ & $s^{-1} t=15 m i n$ & $\begin{array}{c}(3) \\
t=45 \mathrm{~min}\end{array}$ \\
\hline 1 & 1.09 & 1.15 & 1.41 & 2.02 & 1.54 & 1.21 & 1.92 & 0.99 \\
\hline 5 & 1.20 & 1.45 & 2.37 & 3.72 & 2.47 & 1.98 & 2.73 & 1.72 \\
\hline 9 & 1.23 & 1.55 & 2.50 & 4.02 & 2.61 & 2.12 & 2.87 & 1.83 \\
\hline 13 & 1.24 & 1.57 & 2.55 & 4.11 & 2.65 & 2.16 & 2.92 & 1.86 \\
\hline 17 & 1.25 & 1.60 & 2.56 & 4.11 & 2.67 & 2.18 & 2.95 & 1.88 \\
\hline
\end{tabular}

Note: (1) $G=50 s^{-1}, t=30$ minutes

(2) Alum dose=8.0 $\mathrm{mg} / \mathrm{L}, t=30$ minutes

(3) Alum dose $=8.0 \mathrm{mg} / \mathrm{L}, \mathrm{G}=50 \mathrm{~s}^{-1}$ 
Table E-3 - Rate of Head Loss(in/hr) With Depth and Different Influent Particle size Distributions for Filter No. $3(1.00 \mathrm{~mm})$

\begin{tabular}{|c|c|c|c|c|c|c|c|c|}
\hline $\begin{array}{l}\text { Sand } \\
\text { Depth } \\
\text { (in) }\end{array}$ & $\begin{array}{c}\text { (1) } \\
0 \mathrm{mg} / \mathrm{L} \\
\text { Alum }\end{array}$ & $\begin{array}{l}(1) \\
2 \mathrm{mg} / \mathrm{L} \\
\text { Alum }\end{array}$ & $\begin{array}{l}(1) \\
8 \mathrm{mg} / \mathrm{L} \\
\text { Alum }\end{array}$ & $\begin{array}{l}(1) \\
16 \mathrm{mg} / \mathrm{L} \\
\text { Alum }\end{array}$ & $\begin{array}{l}(2) \\
G=25 \mathrm{~s}^{-1}\end{array}$ & $\begin{array}{c}(2) \\
G=100\end{array}$ & $\begin{array}{c}(3) \\
s^{-1} t=15 \mathrm{~min}\end{array}$ & $\begin{array}{l}(3) \\
t=45 \mathrm{~min}\end{array}$ \\
\hline 1 & .026 & .058 & .131 & .166 & .139 & .123 & .17 & .106 \\
\hline 5 & .056 & .124 & .307 & .395 & .322 & .295 & .37 & .272 \\
\hline 9 & .076 & .149 & .373 & .491 & .395 & .350 & .41 & .332 \\
\hline 13 & .095 & .159 & .397 & .529 & .418 & .370 & .43 & .349 \\
\hline 17 & .102 & .165 & .406 & .559 & .430 & .380 & .45 & .356 \\
\hline
\end{tabular}

Note: (1) $\mathrm{G}=50 \mathrm{~s}-1, t=30$ minutes

(2) Alum dose $=8.0 \mathrm{mg} / \mathrm{L}, t=30 \mathrm{minutes}$

(3) Alum dose $=8.0 \mathrm{mg} / \mathrm{L}, G=50 \mathrm{~s}^{-1}$ 